University of South Florida

DIGITAL COMMONS

Digital Commons @ University of

@ UNIVERSITY OF SOUTH FLORIDA

South Florida

USF Tampa Graduate Theses and Dissertations

USF Graduate Theses and Dissertations

$2-12-2010$

\title{
Topology Control in Wireless Sensor Networks
}

Pedro Mario Wightman Rojas

University of South Florida

Follow this and additional works at: https://digitalcommons.usf.edu/etd

Part of the American Studies Commons

\section{Scholar Commons Citation}

Wightman Rojas, Pedro Mario, "Topology Control in Wireless Sensor Networks" (2010). USF Tampa Graduate Theses and Dissertations.

https://digitalcommons.usf.edu/etd/1807

This Dissertation is brought to you for free and open access by the USF Graduate Theses and Dissertations at Digital Commons @ University of South Florida. It has been accepted for inclusion in USF Tampa Graduate Theses and Dissertations by an authorized administrator of Digital Commons @ University of South Florida. For more information, please contact digitalcommons@usf.edu. 
Topology Control in Wireless Sensor Networks

by

Pedro Mario Wightman Rojas

A dissertation submitted in partial fulfillment

of the requirements for the degree of

Doctor of Philosophy

Department of Computer Science \& Engineering

College of Engineering

University of South Florida

Major Professor: Miguel A. Labrador, Ph.D.

Rafael Perez, Ph.D.

Kenneth Christensen, Ph.D.

Wilfrido Moreno, Ph.D.

William R. Stark, Ph.D.

Date of Approval:

February 12, 2010

Keywords: Network Topology, Topology Construction, Topology Maintenance, Connectivity, Sensing Coverage, Connected Dominating Set

(C) 2010, Pedro Mario Wightman Rojas 
To my three muses Astrid, Hillary and Sophia because all of you inspire me to be a little bit better every day, and to Mother Nature for the awesome job she does with her trees which I humbly try to replicate 


\section{Acknowledgements}

The last five years have been part of an experience in which I have gratefully had the opportunity to grow not only as a professional but as a human being.

I will start by thanking my mentor and adviser Dr. Miguel Labrador, who has guided and supported me throughout these years with admirable patience, and who also has become a good friend. Thanks also to all the staff at the Department of Computer Science and Engineering at USF and Universidad del Norte which have been always there for me. Thanks to my committee Dr. Christensen, Dr. Moreno, Dr. Perez and Dr. Stark for accepting my invitation to be part of this experience and for their valuable comments and suggestions.

My deepest gratitude to my wife Hillary and my beloved daughter Sophia, whose love, patience and support have helped to keep me on the right track. I extend this gratitude especially to my mother for her unconditional love, and to my parents-in-law without whose help I would not have had the opportunity to finish this dissertation.

Thanks to all my friends who I no longer consider just friends, because we have become more like a family: thanks to Dala, Migue, and all the people from the Information Systems Lab for their support and the good laughs. Special thanks to Aldo whose help was critical in these final months and to Alcides for the name of the simulator, among other excellent suggestions.

Finally, and most importantly, I thank God for giving me the opportunity to grow and improve myself in order to be able to serve better. 


\section{Table of Contents}

List of Tables vii

List of Figures $\quad$ viii

Abstract $\quad$ xiii

Chapter 1 Introduction 1

1.1 Wireless Sensor Networks 2

1.2 Topology Control 5

1.2.1 Network Topology 5

1.2.2 Definition of Topology Control 8

$\begin{array}{lll}1.3 & \text { Problem Statement } & 11\end{array}$

$\begin{array}{lll}1.4 & \text { Contributions } & 15\end{array}$

$\begin{array}{lll}1.5 & \text { Structure of the Dissertation } & 17\end{array}$

Chapter 2 Literature Review 19

2.1 Topology Control Taxonomy 20

2.1.1 Topology Construction Taxonomy 21

2.1.2 Topology Maintenance Taxonomy 22

2.2 Connectivity-oriented Topology Construction 23 
2.2.1 Controlling the Transmission Power

2.2.1.1 Centralized Approaches

2.2.1.2 Distributed Approaches

2.2.1.3 Heterogeneous Devices

2.2.2 Hierarchical Techniques

2.2.2.1 Backbone-based Techniques

2.2.2.2 Cluster-based Techniques

2.2.2.3 Adaptive Techniques

2.2.3 Hybrid Approaches

2.3 Coverage-oriented Topology Construction

2.3.1 Classification Factors

2.3.1.1 Definition of the Area of Interest

2.3.1.2 Redundancy

2.3.2 Other Considerations in Coverage-oriented Topology

Construction Protocols

2.3.3 Examples of Coverage-oriented Topology Construction Protocols

2.4 Topology Maintenance

2.4.1 Classification Factors

2.4.1.1 Selection Policy: Static, Dynamic and Hybrid

2.4.1.2 Level of Involvement: Global and Local

2.4.1.3 Triggering Mechanisms 
$\begin{array}{lll}\text { Chapter } 3 & \text { Methodology } & 72\end{array}$

3.1 An Analytical Solution to the MCDS Problem 72

3.2 The MIP Approach for the Minimum Connected Dominating Set Problem

3.3 Performance Evaluation: Assumptions, Metrics, Factors and Levels

$\begin{array}{lll}3.3 .1 & \text { Assumptions } & 82\end{array}$

3.3.2 Performance Metrics $\quad 83$

$\begin{array}{lll}\text { 3.3.3 Factors and Levels } & 84\end{array}$

3.3.4 Energy Model $\quad 86$

Chapter 4 The A3 and A3Lite Topology Construction Protocols for Connectivity 88

$\begin{array}{lll}4.1 & \text { Introduction } & 89\end{array}$

$\begin{array}{lll}\text { 4.2 The A3 Algorithm } & 90\end{array}$

4.2.1 The Neighborhood Discovery Process 91

4.2.2 Children Selection Process 92

$\begin{array}{lll}\text { 4.2.3 Second Opportunity Process } & 94\end{array}$

4.2.3.1 The Selection Metric 96

$\begin{array}{lll}\text { 4.3 The A3Lite Algorithm } & 98\end{array}$

4.3.1 The Neighborhood Discovery Process 98

$\begin{array}{ll}\text { 4.3.2 Children Selection Process } & 99\end{array}$

$\begin{array}{lll}4.4 & \text { Performance Evaluation } & 100\end{array}$ 
4.4.1 Experiment 1: Changing the Node Degree

4.4.2 Experiment 2: Changing the Node Density

4.4.3 Experiment 3: Ideal Grid Topologies

108

Chapter 5 The A3Cov and A3CovLite Topology Construction Protocols for Coverage

5.1 The A3Cov Algorithm

5.2 The A3CovLite Algorithm

5.3 The $\alpha$-Coverage Sensing Coverage Definition

5.4 Performance Evaluation

5.4.1 Comparison with Theoretical Deployments

5.4.1.1 Experiment 1: Same Radii

5.4.1.2 Experiment 2: Different Radii

5.4.1.3 Experiment 3: Different $\alpha$-coverage

132

5.4.2 Comparison with Distributed Protocols

5.4.2.1 Comparison With ACOS

5.4.2.2 Comparison With StanGA

Chapter 6 Topology Maintenance Protocols

6.1 Introduction

6.2 Static Global Topology Rotation

6.3 Dynamic Global Topology Recreation

6.4 Dynamic Local - DSR

6.5 Hybrid Global Topology Recreation and Rotation 
6.6.1 Performance Evaluation of Static Global Topology Maintenance Techniques

6.6.1.1 Sparse Networks

6.6.1.2 Dense Networks

6.6.2 Performance Evaluation of Dynamic Global Topology Maintenance Techniques

6.6.2.1 Sparse Networks

6.6.2.2 Dense Networks

6.6.3 Performance Evaluation of Dynamic Local Topology

Maintenance Techniques

6.6.3.1 Sparse Networks

6.6.3.2 Dense Networks

6.6.4 Performance Evaluation of Hybrid Global Topology Maintenance Techniques

6.6.4.1 Sparse Networks

6.6.4.2 Dense Networks

6.7 Comparison of Topology Maintenance Techniques

6.8 Sensitivity Analysis

6.8.1 Time-based Analysis

6.8.2 Energy-based Analysis 
7.1 Conclusions

7.2 Summary of Contributions

7.3 Future Work

List of References

Appendices

Appendix A: A Brief Overview of Atarraya 


\section{List of Tables}

Table 3.1 Parameters for the energy model. $\quad 87$

Table 4.1 Simulation parameters for connectivity-oriented protocols. 101

Table 5.1 Simulation parameters for coverage-oriented protocols. 121

Table 5.2 Radii and topology sizes for coverage-oriented protocols. 123

Table 6.1 Simulation parameters for topology maintenance protocols. 154 


\section{List of Figures}

Figure 1.1 Diagram of components of a wireless sensor device.

Figure 1.2 Example of a network architecture that includes a wireless sensor network.

Figure 1.3 Comparison of topologies: the MaxPowerGraph versus the reduced topology.

Figure 1.4 Diagram that models the iterative execution of a topology control algorithm.

Figure 2.1 General taxonomy for topology control protocols.

Figure 2.2 Taxonomy of connectivity-oriented topology construction protocols.

Figure 2.3 Topology control by reducing the number of active nodes and the creation of a network backbone.

Figure 2.4 Growing a tree with 1-hop neighbor information.

Figure 2.5 Classification of coverage-oriented topology construction protocols.

Figure 2.6 Different deployment geometries for connected coverage topologies with $R_{S}=R_{C}$.

Figure 2.7 Example of original and modified solutions of the circle packing problem.

Figure 2.8 Theoretical comparison between the packing problem and the optimal deployments.

Figure 2.9 Multiple node-disjoint CDS trees over the same network. 
Figure 2.10 Classification of topology maintenance.

$\begin{array}{lll}\text { Figure 3.1 Examples of MDS solutions. } & 73\end{array}$

Figure 3.2 Example of the "flow of tokens" technique. 79

$\begin{array}{lll}\text { Figure 4.1 The A3 algorithm. } & 91\end{array}$

$\begin{array}{lll}\text { Figure } 4.2 \text { The A3 protocol finite state machine. } & 92\end{array}$

$\begin{array}{lll}\text { Figure 4.3 The A3Lite protocol finite state machine. } & 98\end{array}$

Figure 4.4 Results of experiment 1: changing the node degree. 103

Figure 4.5 Results of experiment 2: changing the node density. 106

$\begin{array}{lll}\text { Figure 4.6 Square grids deployment. } & 108\end{array}$

Figure 4.7 Results of experiment 3: ideal grid topologies. 110

$\begin{array}{lll}\text { Figure 5.1 The A3Cov protocol finite state machine. } & 113\end{array}$

Figure 5.2 The A3CovLite protocol finite state machine. 115

$\begin{array}{lll}\text { Figure 5.3 Example of } \alpha \text {-coverage. } & 117\end{array}$

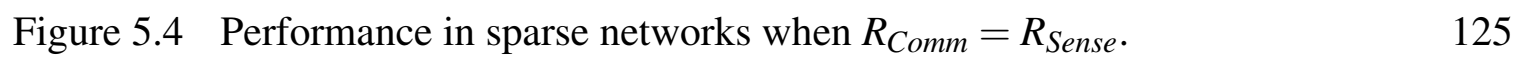

Figure 5.5 Performance in dense networks when $R_{\text {Comm }}=R_{\text {Sense }} . \quad 126$

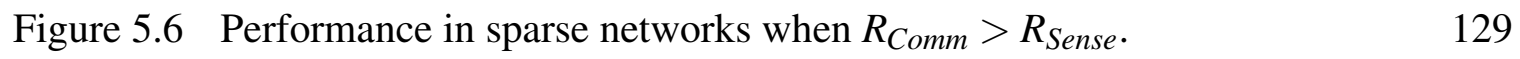

Figure 5.7 Performance in dense networks when $R_{\text {Comm }}>R_{\text {Sense }} . \quad 130$

Figure 5.8 Performance in sparse networks when $R_{\text {Comm }}>R_{\text {Sense }}$ and $\alpha>1 . \quad 133$

Figure 5.9 Performance in dense networks when $R_{\text {Comm }}>R_{\text {Sense }}$ and $\alpha>1 . \quad 134$

Figure 5.10 Performance in sparse networks when $R_{\text {Comm }}>R_{\text {Sense }}$ and $\alpha<1 . \quad 136$

Figure 5.11 Performance in dense networks when $R_{\text {Comm }}>R_{\text {Sense }}$ and $\alpha<1 . \quad 137$ 
Figure 5.12 Performance of the A3Cov and A3CovLite protocols in sparse networks with different radii and $\alpha$-coverage parameter.

Figure 5.13 Performance of the A3Cov and A3CovLite protocols in dense networks with different radii and $\alpha$-coverage parameter.

Figure 5.14 Comparison of performance of the A3Cov, A3CovLite protocols and the ACOS protocol for 800 nodes.

Figure 5.15 Comparison of performance of the A3Cov, A3CovLite protocols and the StanGA protocol for different coverage configurations.

Figure 6.1 Phase one of the DSR-based dynamic local topology maintenance technique.

Figure 6.2 Network lifetime with and without static global topology maintenance using the A3, EECDS, and CDS-Rule-K topology construction mechanisms in sparse networks.

Figure 6.3 Best performing static global topology maintenance techniques in sparse networks.

Figure 6.4 Network lifetime with and without static global topology maintenance using the A3, EECDS, and CDS-Rule-K topology construction mechanisms in dense networks.

Figure 6.5 Best performing static global topology maintenance techniques in dense networks.

Figure 6.6 Network lifetime with and without dynamic global topology maintenance using the A3, EECDS, and CDS-Rule-K topology construction mechanisms in sparse networks.

Figure 6.7 Best performing dynamic global topology maintenance techniques in sparse networks.

Figure 6.8 Network lifetime with and without dynamic global topology maintenance using the A3, EECDS, and CDS-Rule-K topology construction mechanisms in dense networks.

Figure 6.9 Best performing dynamic global topology maintenance techniques in dense networks. 
Figure 6.10 Network lifetime with and without dynamic local topology maintenance using the A3, EECDS, and CDS-Rule-K topology construction mechanisms in sparse networks.

Figure 6.11 Best performing dynamic local topology maintenance techniques in sparse networks.

Figure 6.12 Network lifetime with and without dynamic local topology maintenance using the A3, EECDS, and CDS-Rule-K topology construction mechanisms in dense networks.

Figure 6.13 Best performing dynamic local topology maintenance techniques in dense networks.

Figure 6.14 Network lifetime with and without hybrid global topology maintenance techniques using the A3, EECDS, and CDS-Rule-K topology construction mechanisms in sparse networks.

Figure 6.15 Best performing hybrid global topology maintenance techniques in sparse networks.

Figure 6.16 Network lifetime with and without hybrid global topology maintenance techniques using the A3, EECDS, and CDS-Rule-K topology construction mechanisms in dense networks.

Figure 6.17 Best performing hybrid global topology maintenance techniques in dense networks.

Figure 6.18 Best performing topology maintenance techniques in sparse and dense networks.

Figure 6.19 Time-based sensitivity analysis of network lifetime for static, dynamic, and hybrid topology maintenance techniques.

Figure 6.20 Best performing techniques out of time sensitivity tests.

Figure 6.21 Energy-based sensitivity analysis of network lifetime for static, dynamic, and hybrid topology maintenance techniques.

Figure 6.22 Best performing techniques out of energy sensitivity tests. 
Figure 6.23 Node density-based sensitivity analysis of network lifetime for static, dynamic, and hybrid topology maintenance techniques.

Figure 6.24 Best performing techniques out of the density sensitivity tests.

Figure 6.25 Comparison of network lifetime when using topology construction and maintenance, topology construction only and no topology control.

Figure A.1 Atarraya's functional components.

Figure A.2 Useful diagrams to design a communication protocol.

Figure A.3 Simulation control panel.

Figure A.4 Other protocols for educational purposes.

Figure A.5 Different topology designs generated by Atarraya.

Figure A.6 Deployment definition panel.

Figure A.7 Other parameters for deployment definition.

Figure A.8 Example of statistics generated by Atarraya.

Figure A.9 Report panel.

Figure A.10 Node statistics panel.

Figure A.11 Main window description.

Figure A.12 Visualization control panel. 


\section{Topology Control in Wireless Sensor Networks}

\section{Pedro Mario Wightman Rojas}

\section{ABSTRACT}

Wireless Sensor Networks (WSN) offer a flexible low-cost solution to the problem of event monitoring, especially in places with limited accessibility or that represent danger to humans. WSNs are made of resource-constrained wireless devices, which require energy efficient mechanisms, algorithms and protocols. One of these mechanisms is Topology Control (TC) composed of two mechanisms, Topology Construction and Topology Maintenance.

This dissertation expands the knowledge of TC in many ways. First, it introduces a comprehensive taxonomy for topology construction and maintenance algorithms for the first time. Second, it includes four new topology construction protocols: A3, A3Lite, A3Cov and A3LiteCov. These protocols reduce the number of active nodes by building a Connected Dominating Set (CDS) and then turning off unnecessary nodes. The A3 and A3Lite protocols guarantee a connected reduced structure in a very energy efficient manner. The $\mathrm{A} 3 \mathrm{Cov}$ and $\mathrm{A} 3 \mathrm{LiteCov}$ protocols are extensions of their predecessors that increase the sensing coverage of the network. All these protocols are distributed -they do not require localization information, and present low message and computational complexity. Third, this dissertation also includes and evaluates the performance of four topology maintenance protocols: Recreation (DGTRec), Rotation (SGTRot), Rotation and Recreation (HGTRotRec), and Dynamic Local-DSR (DLDSR). 
Finally, an event-driven simulation tool named Atarraya was developed for teaching, researching and evaluating topology control protocols, which fills a need in the area of topology control that other simulators cannot. Atarraya was used to implement all the topology construction and maintenance cited, and to evaluate their performance. The results show that A3Lite produces a similar number of active nodes when compared to A3, while spending less energy due to its lower message complexity. A3Cov and A3CovLite show better or similar coverage than the other distributed protocols discussed here, while preserving the connectivity and energy efficiency from A3 and A3Lite. In terms of network lifetime, depending on the scenarios, it is shown that there can be a substantial increase in the network lifetime of $450 \%$ when a topology construction method is applied, and of $3200 \%$ when both topology construction and maintenance are applied, compared to the case where no topology control is used. 


\section{Chapter 1: Introduction}

Data is today's gold: finding new sources, new ways to gather it and new kinds of conclusions to draw from it are becoming very attractive research areas with countless applications in the world. In the time you spent reading this dissertation, billions of bits will be generated with the only purpose of gathering data about virtually everything: how many cars are crossing the Skyway bridge in St. Petersburg, FL, the temperature on each floor of the Empire State building in New York city, or the current location of a group of zebras in the savannas of Central Africa, and this is just the beginning. However, gathering this data from places that are not easily accessible or that are dangerous to humans requires the right technology.

One technology that fits the requirements needed for a low-cost and flexible way to obtain data from virtually any location, from urban environments, to personal networks and also scenarios with limited access to communication and power infrastructures is Wireless Sensor Networks (WSNs). As it will be seen later on this chapter, these networks are made of devices very constrained in resources, which makes it imperative for them to work in a very efficient manner, especially in terms of energy consumption. One of the ways a WSN can become more energy-efficient is by using Topology Control (TC), which is the main focus of this dissertation.

This chapter formally introduces the concept of Wireless Sensor Networks and Topology Control. Furthermore, this chapter explains in detail the motivations behind the use of 


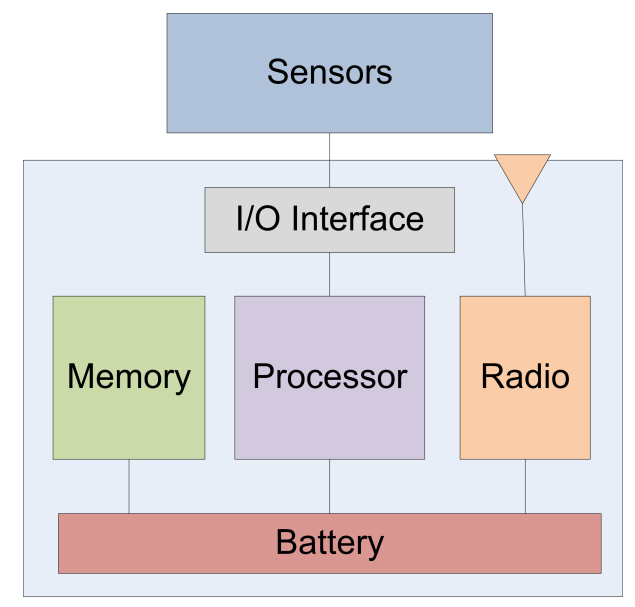

Figure 1.1: Diagram of components of a wireless sensor device.

WSNs and how Topology Control algorithms can improve their performance. Subsequently, the problem statement will be introduced, accompanied by some discussion on the key study variables and procedures used in this work. After the problem statement, the chapter includes the detailed list of contributions presented in this dissertation, and finalizes with the structure of the dissertation.

\subsection{Wireless Sensor Networks}

Advances in sensor and wireless communication technologies in conjunction with developments in microelectronics have made available a new type of communication network made of battery-powered integrated wireless devices with sensing capabilities. Wireless Sensor Networks, as they are named, are self-configured and infrastructureless wireless networks made of small devices equipped with specialized sensors, wireless transceivers, a processing unit, a small memory unit, and a power source, which can be as small as a pair of AA batteries. A general diagram of the structure of one of these devices can be seen in Figure 1.1, taken from [1]. 


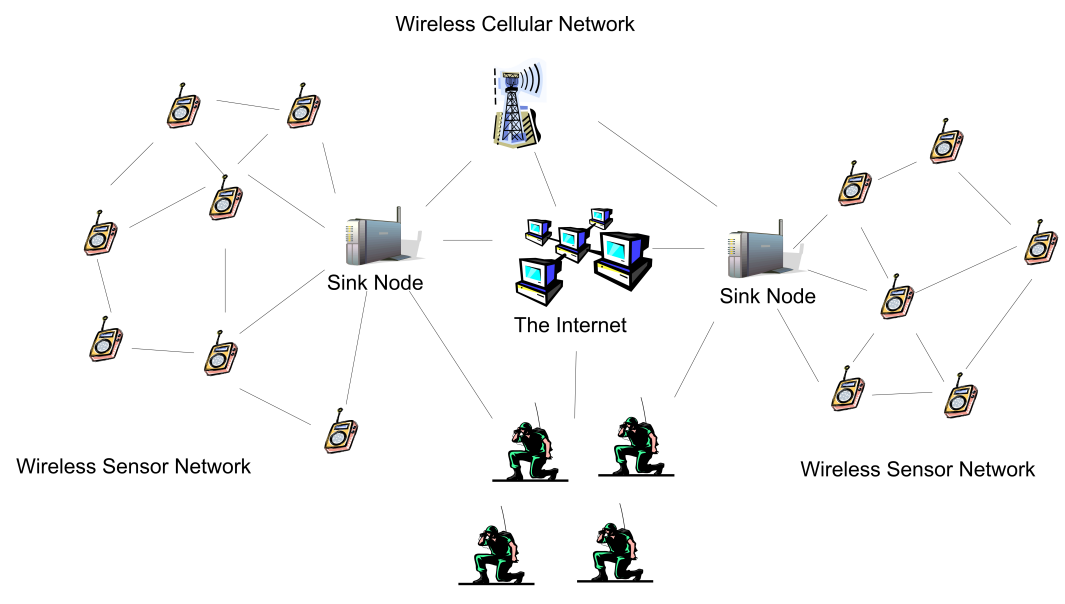

Wireless Ad Hoc Network

Figure 1.2: Example of a network architecture that includes a wireless sensor network.

The main goal of a WSN is to collect data from the environment and send it to a reporting site where the data can be stored, observed and analyzed. Wireless sensor devices also respond to queries sent from a control site to perform specific instructions or provide ondemand sensing samples. Finally, wireless sensor devices can be equipped with actuators to perform actions upon certain conditions. These networks are sometimes more specifically referred as Wireless Sensor and Actuator Networks.

Some of the applications in which the use of a WSN has been ideal include the monitoring and acting upon events in dangerous or unaccessible places for humans. As such, WSNs have been installed in places such as chemical plants, to monitor poisonous gases; water plants, rivers, lakes and the like, to assess the level and quality of the water; areas with endangered species, to monitor their travel patterns and behaviors; buildings, to monitor the quality of the air and make them more energy-efficient; used by the military, to detect intruders; or used in other similar applications.

Figure 1.2 presents an example of a complete solution that integrates WSNs with other current technologies, like the cellular network, the Internet, and other wireless ad hoc 
technologies [1]. As it can be seen, a typical structure of a WSN includes two types of wireless devices: sink nodes and regular nodes.

The sink nodes are the gateways of the WSN: all data generated from the sensor network will be gathered at the sink node and transmitted to the control site using a secondary communication interface, like Ethernet, cellular, satellite or another wireless network. Furthermore, the sink nodes also allow information from outside into the WSN, like commands, updates or queries. In some cases, the sink nodes also play the role of organizers of the network, keeping track of the state of the nodes and address assignation, or by being the initiators of the maintenance procedures.

The regular nodes are the gross majority in the network; they are in charge of collecting all the data about the variables being monitored and of reporting it to the sink node. In addition, if the network is large enough that some devices cannot reach the sink node directly, the regular nodes must provide multi-hop forwarding of the data, so that even the farthest nodes can send their data to the sink node. Due to the critical responsibilities of the sink nodes, they often have a better configuration in terms of processing, memory and, mainly, energy, when compared to the regular nodes; in other words, when every regular node can fail, the sink nodes are expected not to.

Even though the application domain of WSNs has been restricted to simple data-oriented monitoring and reporting applications, especially because of the energy constrained characteristics of the current technology, new network architectures with heterogeneous devices and expected advances in technology are eliminating some of the current limitations and expanding the spectrum of possible applications for WSNs considerably, including more advanced functions like handling multimedia data.

However, at the present time, of all the constraints considered in most available wireless sensor devices, energy consumption is of paramount importance. This assertion can be 
explained with the following two reasons: first, a single wireless device possesses a small energy source, which is expected to last several months; and second, if the network is deployed in an inaccessible area, changing depleted batteries is not feasible, so the wireless devices must use their already small energy source in a very efficient way. These are the main reason why most of the research on WSNs has been concentrated on the design of energy- and computationally-efficient algorithms and protocols; this interest can be measured by the large number of algorithms, techniques, and protocols that have been developed to save energy, and thereby extend the lifetime of the network. One of the most important techniques utilized to reduce energy consumption in wireless sensor networks is Topology Control.

\subsection{Topology Control}

\subsubsection{Network Topology}

Before the concept of Topology Control is introduced, as well as all its benefits for the network's operations, it is important to start by dedicating some time to the concept of Network Topology, which is absolutely necessary to understand the foundations and motivations behind TC. A simple definition of network topology can be the following:

Network Topology is the set of all active nodes and active links in the network along which direct communication can occur [2].

This definition can be extended based on the concept of Geometric Random Graphs. Let $G=(V, E, r)$ be a Geometric Random Graphs (GRG), in which $V$ is the set of vertices, $E$ is the set of edges, and $r$ is the radius of the transmission range of the nodes. Every vertex on $V$ represents a wireless sensor device, and has a geometric coordinate associated to it 
and an open ball with radius $r$. This open ball is a set that contains all the vertices with distance less than $r$ respective to the node, which represents the communication area of the nodes. The nodes in the open ball are the only neighbors that the node can communicate with directly. The formal definition of the open ball is presented in Equation 1.1 [3] as follows:

$$
B_{r}(x)=\{y: d(x, y)<r\}
$$

where $x, y \in V$ and $d(\cdot, \cdot)$ is the Euclidean distance between two nodes. The set of edges $E$ are the union of all pairs created by each vertex and all the adjacent vertices contained in its open ball. Providing that communication is not always bidirectional, links are modeled as unidirectional edges. Given that the open balls of the vertices are independent, the existence of the edge $(x, y)$ does not imply the existence of the edge $(y, x)$. In addition, if any metric can be calculated between a pair of adjacent nodes, i.e., distance, angle, remaining energy, etc., it can be used as the weight of the edge, $w$. This metric, as well as the edges, is not always symmetric, so weights could be different from one direction to the other. The formal definition of the set of edges is presented in Equation 1.2. However, it is assumed that all networks used in this dissertation can be modeled as bidirectional graphs.

$$
E=\left\{(x, y, w): y \in B_{r}(x) \wedge w \in \Re\right\}, x, y \in V
$$

The resultant graph when all the sensors are set to transmit at their maximum power is called the Maximum Power Graph, or MaxPower graph. This is normally the case when the nodes have just been deployed and they are exploring their neighborhood. This graph represents the maximum topology of the network in terms of active nodes and active 


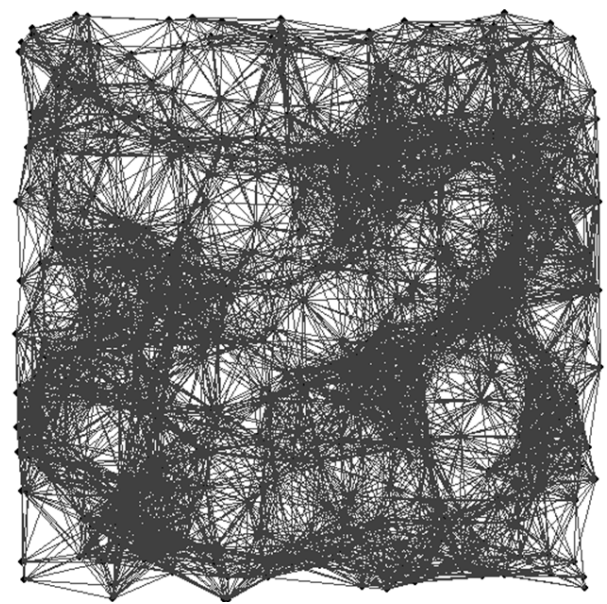

(a) The Maximum Power Graph.

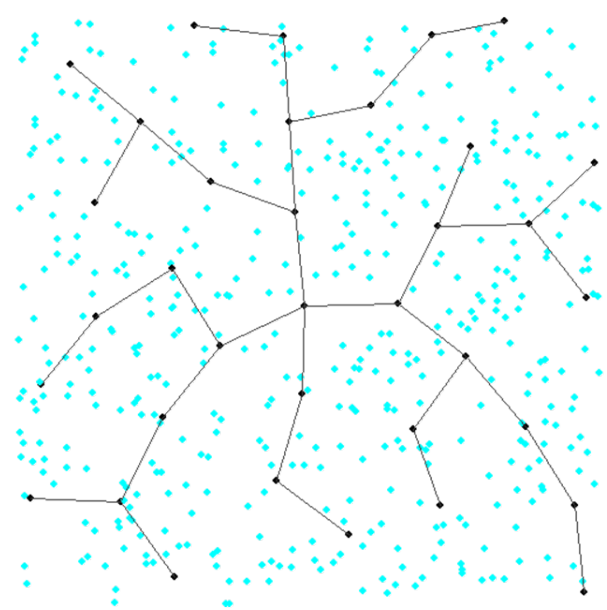

(b) The reduced topology after the topology construction mechanism.

Figure 1.3: Comparison of topologies: the MaxPowerGraph versus the reduced topology.

links. One example of this graph is shown in Figure 1.3(a), in which 500 nodes are uniformly deployed in an area of $500 m \times 500 m$ with transmission ranges of $80 m$.

In terms of the organization of the a network topology, it can be predesigned by the user in the cases where manual allocation of the nodes is possible, or it can be totally random in the cases where the deployment is made by other means, i.e., the nodes are dropped from a helicopter.

In the first case, the user has total control over the topology: the user can calculate the minimum amount of required nodes and their exact positions on the area in order to perform the task in an optimal manner. This technique is not feasible for applications in which accessibility is not possible or in a dangerous scenario where the lives of the people performing the deployment are at risk. In addition, this solution many not be feasible for large networks or when immediate availability of the network is required.

In the second case, when the topology is randomly deployed on an inaccessible area, the user lacks the power to assign exact locations to the nodes and needs a higher number of nodes in order to guarantee coverage of the area. These issues create another problems, 
namely a random topology configuration that is not apt for optimal performance, which can show characteristics such as:

- There can be very dense areas in which many nodes are transmitting packets very often, generating a great amount of collisions and delays.

- The nodes may be transmitting at full power, which is not necessary to reach the next node in their path towards the sink.

- There are many nodes that, due to proximity, are sensing the same events and thus, sending redundant data to the sink.

In all of these cases, the network is wasting energy in unnecessary actions. These are the problems that Topology Control can help to prevent.

\subsubsection{Definition of Topology Control}

The main goal of topology control is to modify the initial maximum power topology and avoid the occurrence of the previously mentioned problems and their impact on the energy consumption by altering the initial topology, while keeping important characteristics like connectivity and coverage.

If the user wants to pre-design a topology to guarantee optimality, the need of topology control is not critical and the solution of the optimal topology can be calculated off-line and replicated in reality. However, a solution of this nature should take into account the characteristics of the terrain, the radio spectrum and other variables that may affect the actual communication between nodes once they have been deployed, but sometimes that information is difficult to obtain and the permanent testing, the manual deployment and 
the large number of possible combinations necessary to generate a successful output may delay the whole deployment process.

The second case is when the deployments are random topologies and the location of the nodes cannot be changed or sometimes not even determined. In this scenarios there are still two variables that the user can use to reorganize the topology: the state of activity of the nodes (active, inactive) and the radio transmission power. The first variable allow the user to reduce the number of active nodes, which has an impact on the density in certain areas, reducing interference and the generation of redundant data. Inactive nodes turn off their transceivers and go into a very low energy consumption mode, from which they can be turned on again to be part of the active network if they are needed in the future. The second variable has a direct impact on energy consumption and on the level of interference, given that radio transmission is the most expensive operation in terms of energy and one of the most commonly done; in other words, reducing the energy required to transmit a message will represent important savings.

The advantage of a random topology is that the deployment can be done relatively quickly, and the network may become available almost immediately. In addition, a topology control algorithm should be robust enough to take care of the characteristics of the deployment area. The main disadvantage of this kind of deployment is that a higher amount of nodes is required in order to increase the probability of having nodes in every region of the monitored area, which has a direct impact on the cost of the network.

One example of the application of topology control can be seen in Figure 1.3(b), which shows how the MaxPower network shown in Figure 1.3(a) can be reduced after applying a TC algorithm to decrease the number of active nodes. A more formal definition of topology control can be the following: 
"Topology Control is the reorganization and management from time to time of certain node parameters and modes of operation to modify the topology of the network, with the goal of extending its lifetime while also preserving important characteristics, such as network connectivity and sensing coverage." [1].

In general, TC can be seen as an iterative process, as shown in Figure 1.4, extracted from [1]. First, there is an initialization phase common to all wireless sensor network deployments. In this phase, nodes discover themselves and use their maximum transmission power to build the initial topology. After this initialization phase, the second phase builds a new (reduced) topology. This phase is called Topology Construction. The new reduced topology will run for certain amount of time, as the participating sensors will consume their energy over time. Therefore, as soon as the topology construction phase establishes the reduced network, the Topology Maintenance phase must start working. During this phase, a new algorithm must be in place to monitor the status of the reduced topology and trigger a topology restoration process when appropriate, that may be a process entirely defined by the maintenance protocol itself or that may include the invocation of the topology construction algorithm. Over the lifetime of the network, it is expected that this cycle will be repeated many times until the energy of the network is depleted. There are many different algorithms that can be used in the topology construction and maintenance phases. In this dissertation, new topology construction and topology maintenance protocols will be introduced. 


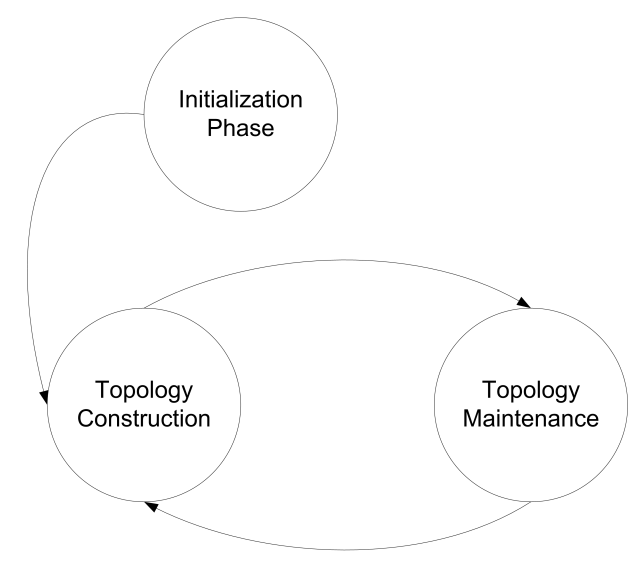

Figure 1.4: Diagram that models the iterative execution of a topology control algorithm.

\subsection{Problem Statement}

The outcome seen in Figure 1.3(b) looks very simple: "Yes, it's a tree that covers everyone. So, is that all?", well, let the following explanation show why the problem of topology control is not as simple as it looks.

First of all, the algorithms and protocols should run in a distributed manner, so they can be implemented in large networks. Second, topology construction algorithms and protocols must have a low computational and message complexity, so they can be efficiently run in computationally weak devices and not drain the nodes' batteries. Third, it is desirable that the algorithms are able to run without the help of additional hardware like GPS devices or localization mechanisms, so low cost is maintained and no additional energy is spent. Finally, the topology construction algorithm must produce a connected network that will cover the area of interest with a minimum number of nodes, while the topology maintenance algorithms must guarantee that the resources of the network are used effectively in order to keep the network active the longest possible time. All these constraints combined make the design and implementation of simple distributed topology control algorithms a very challenging problem. 
In order for these processes to be effective and provide the expected results in terms of extending the lifetime of the network, both topology construction and maintenance mechanisms must be designed with careful consideration of the following requirement aspects:

- Distributed algorithm: Centralized topology control mechanisms need global information and therefore are very expensive when implemented.

- Local information: Nodes should be able to make topology control decisions locally. This reduces the energy costs and makes the mechanism scalable.

- Location information: The need of extra hardware, like GPS devices, or support mechanisms like localization protocols add to the cost in terms of dollars and energy consumption.

- Connectivity: The reduced network must be connected, so all active nodes can exchange messages among themselves as well as with the sink node.

- Coverage: The reduce topology must cover the area of interest despite the number of active nodes.

- Small node degree: A small node degree means a small number of neighbors, which may produce a lower number of collisions and retransmissions, saving energy.

- Link bidirectionality: Bi-directional links facilitate the proper operation of some Medium Access Control (MAC) layer protocols, such as the one utilized by the IEEE 802.11 standard, which sends Request-To-Send (RTS) and Clear-To-Send (CTS) signals and also acknowledgments in the return path.

- Simplicity: Topology control algorithms must have a low computational complexity, so they can be run in wireless sensor devices. 
- Low message complexity: Topology control mechanisms must work with very low message overhead, so they are energy-efficient and can be run many times as part of the topology control cycle.

- Energy-efficiency: All the factors considered thus far and those discussed in the last section converge in the issue of energy-efficiency, which is essential for topology control mechanisms and wireless sensor networks in general.

- Energy awareness: The decision making process in the selection of the reduced topology must be aware of the nodes's remaining energy in order to avoid giving responsibilities to weak nodes that can jeopardize the activity of the network.

- Spanner: The reduced topology should be a spanner of the Unit Disk Graph in terms of both length and number of hops. A subgraph $G^{\prime}$ is a spanner of a graph $G$ for length (number of hops) if there is a positive real constant $x$ such that for any two nodes, the length (number of hops) of the shortest path in $G^{\prime}$ is at most $x$ times of the length (number of hops) of the shortest path in $G$. The constant $x$ is called the length (number of hops) stretch factor.

- Convergence time: The topology construction and maintenance processes should take place as fast as possible and converge after a limited number of steps.

- Memory consumption: Wireless sensor devices often have a small amount of memory. Some topology control techniques may require considerable amounts of memory, such as those that store pre-calculated topologies.

- Even energy distribution: Topology control techniques should somehow try to distribute the energy consumption in an even manner among all the nodes in the 
network. The topologies should be changed so that all nodes have a similar participation in the network.

This dissertation presents new topology control mechanisms that work considering these aspects. All the topology construction protocols proposed in this document work by finding a minimum set of active nodes, that provides a connected network that is able to perform all the tasks required by the user, whether connectivity or coverage, while turning all the rest of the nodes off to save their energy for future maintenance of the active topology. The calculation of this set of special nodes is modeled using the Minimum Connected Dominating Set (MCDS) problem for communication tasks, and the Minimum Connected Sensing Coverage (MCSC) problem for connectivity and coverage, which will be explained in detail in the next chapter.

The main reason why this methodology was selected is that, next to radio transmission, idle listening is the most costly operation of a node. This means that, if all the nodes are kept awake and the only change is made to the transmission power, the idle listening time from the entire network will still decrease the amount of saved energy; plus the fact that the problem of redundant sensing is still present, which will increase the load of the network with no need.

In the proposed solutions of this work, all redundant nodes have their transceivers turned off, so no energy is wasted in idle mode and unnecessary redundancy is reduced. The hypothesis is that the algorithms proposed in this dissertation, while creating minimal message overhead compared to their counterparts, can reduce and maintain an active topology, extending the lifetime of the network, while keeping radio connectivity and sensing coverage of the deployment area. 


\subsection{Contributions}

The contributions presented in this dissertation are the following:

- New taxonomies for topology control, topology construction and topology maintenance algorithms. Based on the new definition of topology control, which differentiates the construction and maintenance processes, a new taxonomy for topology control algorithms was proposed in order to integrate and extend the current algorithms presented in $[2,4,5]$ which focus only on the first process. The main contribution in this area is the definition of a taxonomy for topology maintenance algorithms for the first time, which none of the cited works include. In addition, some modifications were performed on the existing taxonomies, in order to include special branches to topology construction protocols for heterogeneous networks and coverage-oriented protocols.

- The A3 family of simple topology construction algorithms. In this dissertation, four new topology construction algorithms are introduced: A3, A3Lite, A3Cov and A3CovLite. These protocols calculate a Connected Dominating Set (CDS) on the initial MaxPower topology, leaving in active state only the dominating nodes which provide connectivity and coverage in the network, and turning off all dominated (redundant) nodes, which are considered unnecessary for the correct activity of the network at the time of execution. A3 is the first version of the algorithm and provides a backbone for connectivity purposes only. The initial protocol was eventually modified into two new branches: the Lite versions and the Cov versions. The Lite versions provide a very low computational and message complexity, compared to the Non-Lite counterparts. The Cov versions produce a reduced topology that provides a higher degree of area coverage compared to the Non-Cov versions. The 
evaluation of these protocols is based on the comparison of average behavior in random topologies against other algorithms in the literature, and against theoretical optimal deployments.

- A set of topology maintenance algorithms. Four topology maintenance algorithms are presented in this document: Dynamic Global Topology Recreation (DGTRec), Static Global Topology Rotation (SGTRot), Hybrid Global Topology Recreation and Rotation (HGTRecRot) and Dynamic Local DSR, DLDSR. The first two protocols have been proposed before in the literature, but have not been implemented in detail. The last two are completely new algorithms. These algorithms were designed based on the proposed taxonomy for topology maintenance algorithms. The performance evaluation of these algorithms is based on the comparison of the average results in random topologies, and working jointly with three different topology construction protocols. It is very important to mention that, to the knowledge of the author, this is the first time that a modularization of the topology constructions and maintenance protocols has been implemented for joint testing performance.

- Atarraya: a new simulation tool for teaching and researching topology control algorithms. As a tool was needed to design and test the proposed topology control algorithms, the topology control simulator Atarraya was created. Atarraya is a discrete-event simulator for evaluating topology control algorithms. It was developed in Java, which allows Atarraya to be a very portable application among different operating systems. In addition, the simulator is being offered for free to the scientific community as an open source project, based on the GNU license model. This simulator provides a friendly environment for designing both topology construction and maintenance algorithms, allowing the modularity to combine those protocols, which has not been seen in any other simulator of its kind. In addition, 
it can be extended to support schemes for data aggregation, routing, node mobility and different energy models.

- A new book on topology control. Most of the work presented in this dissertation has been included in the book "Topology Control in Wireless Sensor Networks - with a companion simulation tool for teaching and research", written by Miguel Labrador and Pedro Wightman, and published in 2009 by Springer Science [1]. This book presents an integral definition of topology control, based on the decoupling of the construction and maintenance processes, and also provides the simulator Atarraya as a tool not only for researching and designing of new TC protocols, but also as a useful tool for teaching communication protocols in an user-friendly environment.

- A new mixed integer programming definition of the minimal connected dominating set problem. Even though there has been previous definitions of the MCDS problem in the literature, most of them depend on the separate solution of the problems of connectivity and domination, which may not necessarily guarantee an optimal joint solution, or in extensive preprocessing. This dissertation provides an definition of the problem based on a very simple insight of the problem that solves both connectivity and dominance in a single formulation, and does not require any preprocessing. The results of this new model are compared with the results from the approximation protocols presented in Chapter 3 .

\subsection{Structure of the Dissertation}

The structure of the dissertation will continue as follows. Chapter 2 will be dedicated to the literature review of the problem of topology control, including the introduction to the proposed taxonomy, and references to the most important algorithms in both topology 
construction and topology maintenance in the categories of the taxonomy. In addition, a section of the chapter will be dedicated to the Connected Dominating Set-based protocols for topology construction for both connectivity and coverage. Chapter 3 describes the methodology used for the performance evaluation of the different topology construction and maintenance protocols introduced in this dissertation, including both the analytical and simulation-based approaches. Chapter 4 will introduce the topology construction algorithms A3 and A3Lite, which are focused only on producing a connected topology with a minimum number of active nodes, and will show their performance evaluation against two very well known CDS-based topology construction algorithms. The A3Cov and A3CovLite topology construction algorithms will be introduced in Chapter 5, which provide not only a connected topology but a topology that covers a high degree of the deployment area, and will show their performance evaluation against some optimal theoretical deployments, their Non-Cov versions and two other coverage-oriented topology construction protocols. Chapter 6 will present the topology maintenance algorithms proposed in this dissertation, including their joint performance testing with some of the topology construction algorithms presented in Chapter 4. The conclusions of this work and some final remarks will be presented in Chapter 7. In addition, a detailed description of the internal structure and use of the simulator Atarraya will be included in the Appendix A. 


\section{Chapter 2: Literature Review}

The study of topology control has gained importance since the end of the previous decade, especially in wireless ad hoc and sensor networks, because of all the potential benefits in energy consumption that come with transforming the MaxPower graph into a more manageable and efficient network. Researchers in this area took advantage of the similarities of these networks with the random graphs on theoretical fields, in order to adopt the existing bast theory in graphs and apply it into this new kind of network.

This fact determined that the first attempts to perform topology control were based mainly in classical graph algorithms, like the minimal spanning tree, the set cover and coloring problem, just to mention some, because the were already capable of altering the original structure of a graph in order to reduce the topology.

However, most of these algorithms require global information and work in a centralized manner, which became a natural initial assumption to make in order to allow the use of these solutions. These assumptions stopped being valid very quickly for scenarios in which the size of the network made it infeasible to use a centralized solution or when global information was to expensive to obtain. This opened the horizon for the development of distributed protocols that required local information, which corresponded more with some of the real characteristics of these networks: they can reach high levels of scalability, randomness in localization of the nodes and their communication links, and global information is too costly to collect and distribute. 
The amount of work in the area has been very broad and varied. As part of this work, a new taxonomy for classifying topology control protocols is presented. The new proposed taxonomy is an extension of three previous taxonomies, introduced in $[2,4,5]$. The new taxonomy, which is one of the main contribution of this work, provides a road map for the rest of this chapter.

\subsection{Topology Control Taxonomy}

As mentioned in Chapter 1, topology control algorithms were traditionally considered as a monolithic process: reduction and maintenance were implemented as a single protocol. The main problem with this approach is that usually the maintenance process was not assumed critical in the design of the algorithm, so no tests were performed in order to determine the best maintenance policy for the reduced topology produced by the algorithm. In addition, this conception also affected the classification of topology control algorithms, restricting it only to how the network topology was reduced. The two previously defined taxonomies for topology control algorithms covered the following areas:

- The taxonomy presented in [4] is focused only on topology construction algorithms that change the transmission range to reduce the network topology.

- The taxonomy presented in [2] has a broader definition of topology construction algorithms, considering also hierarchical and hybrid algorithms.

- The taxonomy presented in [5] is focused only in the coverage-oriented topology construction protocols. 


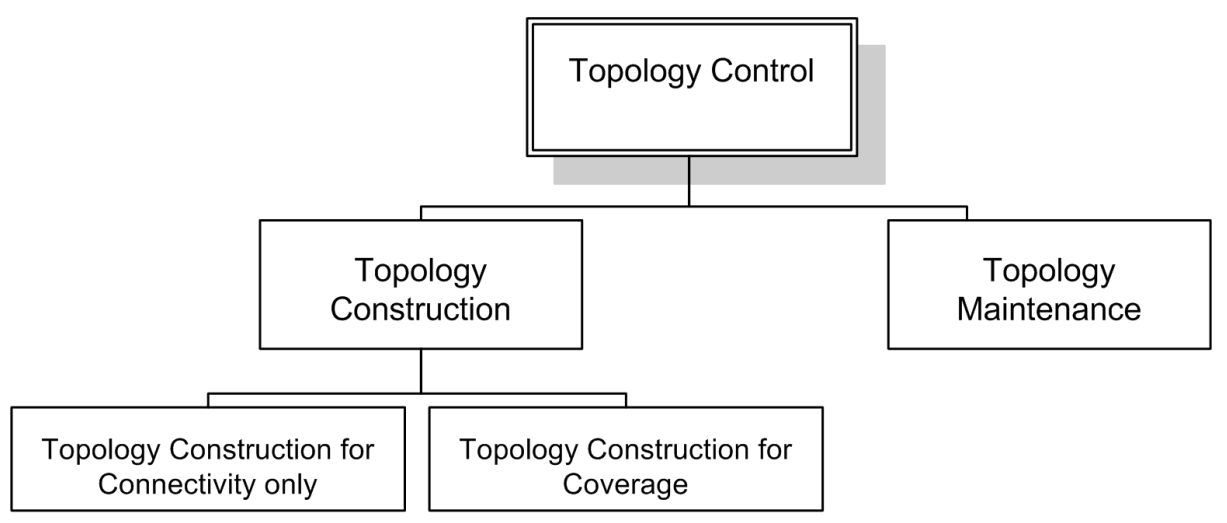

Figure 2.1: General taxonomy for topology control protocols.

The union of these three taxonomies produces a fairly complete taxonomy for topology construction algorithms only. However, some topology construction areas were not explicitly classified in those works, such as networks with heterogeneous devices. The proposed taxonomy not only extends the area of topology construction in the aforementioned area, but also includes a new taxonomy for topology maintenance protocols, which was completely ignored in previous taxonomies. The inclusion of this new branch is motivated by the fact that the selection of a topology maintenance protocol has serious implications on the lifetime of the network, so in order to study the impact of maintenance on topology construction protocols, a clear classification of the different methods to perform maintenance is a necessity. Figure 2.1 depicts the classification of topology control mechanisms into two main branches: Topology Construction and Topology Maintenance.

\subsubsection{Topology Construction Taxonomy}

The first branch of the taxonomy defines the topology construction techniques and divides it into two branches: topology construction for connectivity and topology construction for 
coverage. The algorithms in the first branch are focused only in producing a connected reduced topology, but do not guarantee the correct level of coverage of the deployment area. The protocols in the second branch are more oriented in providing coverage in the area, even if, in some cases, connectivity is not guaranteed.

The following list illustrates a general description of the different techniques used by most of the the connectivity-oriented topology construction protocols:

- Some solutions build a reduced topology by controlling the transmission power, for both homogeneous and heterogeneous networks. Both centralized and distributed techniques are presented.

- Some protocols build hierarchical topologies by means of backbones and clusters.

- Other protocols use hybrid schemes, mixing different techniques in order to reduce the topology.

In contrast, the following list enumerates some of the different techniques used by coverage-oriented topology construction protocols:

- Some protocols are designed to provide coverage of a set of predefined targets distributed in the deployment area, and not the entire area

- Some solutions can offer different levels of redundancy in the coverage

\subsubsection{Topology Maintenance Taxonomy}

The second main branch in the proposed taxonomy is dedicated to topology maintenance protocols. The classification dimensions of the techniques in this category are based on the following parameters: 
- Selection of the nodes for the maintenance problem: pre-calculated static topology selection, "on the fly" dynamic selection or a hybrid selection scheme.

- Level of involvement of the nodes in the maintenance process: global involvement, when all the nodes in the network participate on the algorithms, or local involvement when just a small subset of them perform the maintenance.

- Triggering mechanism of the maintenance algorithm: time, energy, node density, random selection, node failure, etc.

The following section will illustrate some of the characteristic techniques in each of the categories of the taxonomy, giving a higher priority to the hierarchical protocols in the topology construction branch, given that the topology construction protocols presented in this dissertation belong to that category.

\subsection{Connectivity-oriented Topology Construction}

Topology construction, as explained in Chapter 1, is the first process that is executed over the network once the network has been deployed. The main goal of these algorithms is to reduce the topology of the network, while keeping the network connected. The reduction of the topology brings benefits to the network, mainly in energy consumption.

Even though there are many ways to perform such tasks, each protocol in this category modify differently the available parameters -the transmission power and the level of activity of the nodes; and uses different information to make decisions -node location, number of neighbors, etc. This section is focused on the description of the most important types of topology construction algorithms, with example algorithms for each category of the detailed taxonomy presented in Figure 2.2. 


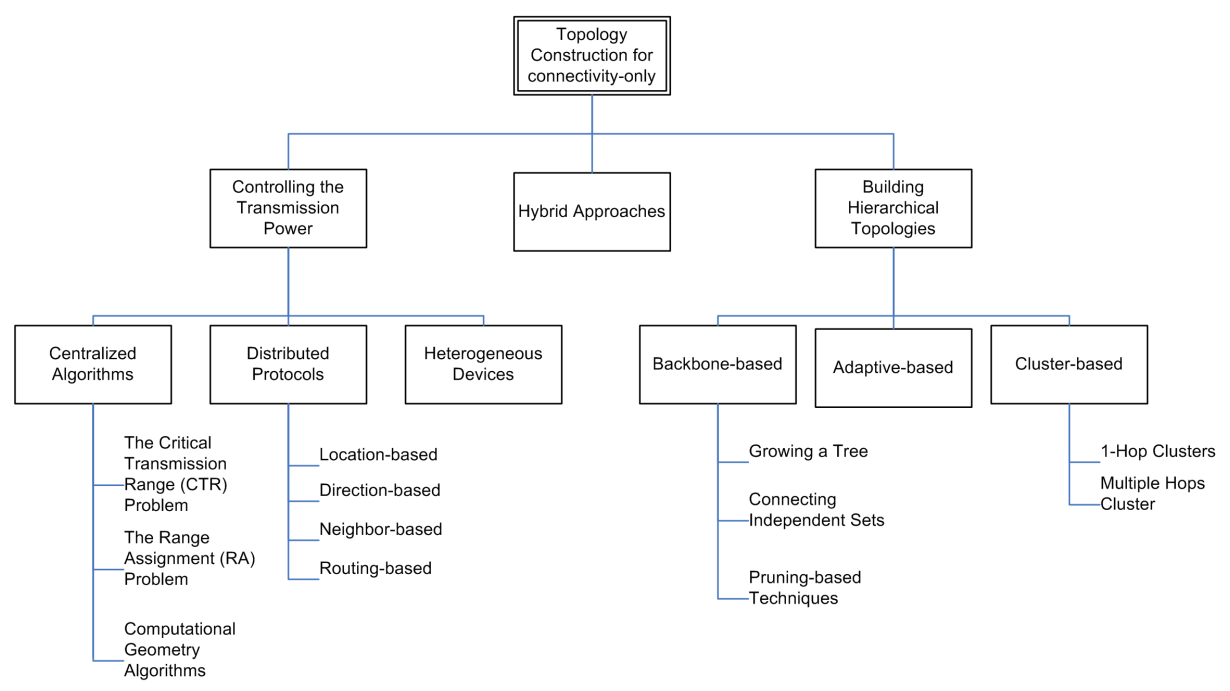

Figure 2.2: Taxonomy of connectivity-oriented topology construction protocols.

\subsubsection{Controlling the Transmission Power}

This section describes the most important topology construction algorithms and protocols that build the reduced topology by controlling the transmission power of the nodes. The first case considered in this section is one where all nodes in the network are similar, i.e., assuming an homogeneous infrastructure. For this case, centralized algorithms and distributed protocols are presented. The second case includes protocols that consider a network with heterogeneous devices, in terms of energy and transmission range. This case will be explained with more detail later in Section 2.2.1.3.

\subsubsection{Centralized Approaches}

Controlling the transmission power in a centralized manner is perhaps the most mature topology construction technique, given that many techniques from classical graph theory 
could be applied almost directly. Under the centralized paradigm there are three wellknown approaches:

- Finding the minimal communication range for all the nodes in the network that will preserve network connectivity. This approach is better represented by the problem of the Critical Transmission Range (CTR).

- Finding the optimal transmission power for each individual node, which is the Range Assignment (RA) problem.

- The use of geometrical properties in order to find reduced global topologies based on local optimal solutions

The first approach was presented in [6]. This idea is based on the idea that a Minimal Spanning Tree (MST) on a graph provides connectivity. If the communication range of every node is guaranteeing to be as large as the longest edge of the MST, it will imply that the network is connected. In this sense, Penrose determined that for dense networks, the length of the longest edge, from which the value of the CTR can be calculated, is determined with high probability (w.h.p) by Equation 2.1.

$$
C T R_{\text {dense }}=\sqrt{\frac{\log n+f(n)}{n \pi}}
$$

where $f(n)$ is an increasing function of $n$, such that $\lim _{n \rightarrow \infty} f(n)=+\infty$, and $\log n$ is the natural logarithm of $n(\ln n)^{1}$.

Equation 2.1 is valid only for two-dimensional deployments. Other similar formulas can be seen in [4] for one- and three-dimensional deployments, based on theorems from [68]. However, this equation has several limitations. First, it only applies to dense networks.

\footnotetext{
${ }^{1}$ Unless otherwise specified, all logarithms in this section are natural logarithms.
} 
The asymptotic value of the longest edge is found in a fixed area as the number of nodes goes to infinity. Second, it is not very accurate. Experiments in $[1,4]$ show that even for a large number of nodes, the theoretical value given by Equation 2.1 has a relative difference in the order of $28 \%$ when compared with experimental results.

Another formula was presented in [9] which guarantees connectivity in both sparse and dense networks. It uses the length of the side of the deployment area (assuming that it is a square), and calculates the optimum radius and number of nodes to obtain a fully connected sparse network. For the one-dimensional case, the CTR is given by:

$$
C T R=k \frac{\log l}{n}
$$

where $k$ is a constant with $1 \leq k \leq 2$, and $l$ is the length of the line. The asymptotic behavior of this CTR has been shown to be very accurate when compared with experimental results in [4] for $k=1$, if certain assumptions are considered. For instance, the relative magnitude of the transmission range $r$ and the number of nodes $n$ when expressed as a function of the length of the line $l$ have to be such that $r=r(l)<<l$ and $n=n(l)>>1$. The authors also provided a partially proven formula for d-dimensional cases.

For d-dimensional cases, with $d=2,3, \ldots$, Santi [4] proposes a partially proved result to find the CTR for connectivity as:

$$
C T R=k \frac{l^{d} \log l}{n}
$$

where $k$ is a constant with $0 \leq k \leq 2^{d} d^{\frac{d}{2+1}}$.

In general, the procedure to find the Critical Transmission Range may be a hard and costly operation, especially if complete connectivity is desired. Also, in some cases, the CTR 
might be very close to the maximum transmission range, and therefore, there will be neither major differences in the topology nor energy savings.

If the homogeneous range constraint is removed, the solution utilized to find the CTR can provide an even better solution. A more interesting and energy-efficient method would be to find the maximum energy needed per node; in other words, a non-homogeneous power assignment to build a reduced connected topology without the drawbacks of having an homogeneous range. This is the second approach on the centralized paradigm: the wellknown Range Assignment (RA) problem, which is defined as the function that finds a strongly connected graph and minimizes the total cost of the network, which is given by the summation of the transmission power used by all $n$ nodes.

The algorithm presented in [10] to solve the RA problem has a computational complexity of $O\left(n^{4}\right)$ for one-dimensional networks. Solving the RA problem in two- and threedimensional networks is NP-hard $[10,11]$. Therefore, symmetry constraints have been added to the RA problem, and two new problems emerged: The Weakly Symmetric Range Assignment (WSRA) problem and the Symmetric Range Assignment (SRA) problem. The solution to the Weakly Symmetric Range Assignment (WSRA) problem removes unidirectional links and determines the range assignment for each node such that the reduced graph is connected and symmetric, and the total cost of all the assignments is minimum. Note that in the case of the WSRA problem, the resulting topology may still contain some unidirectional links, which are not needed for connectivity. The Symmetric Range Assignment (SRA) problem, on the other hand, is stronger in its requirements, as all links in the resulting topology must be bidirectional. This implies that some nodes will have to increase their transmission power to produce the symmetric topology. The WSRA problem appears to be more convenient to solve in wireless sensor networks because it presents the same computational complexity as the other solutions, and it cre- 
ates a connected backbone of bidirectional links, which is what nodes need to communicate. In addition, WSRA has been proved in [4] that the energy cost of the WSRA problem has a marginal additional energy cost compared with the RA problem, and a great gain compared to the SRA.

The third approach takes advantage of three very well known algorithms that are based in geometrical properties of the Euclidean space: the Relative Neighbor Graph $(R N G)$ [12], the Gabriel Graph $(G G)$ [13] and the Delaunay Triangulation (DT) [14]. It was decided to include these algorithms in this section of the taxonomy because in their original versions they work in a centralized manner, even though several distributed implementations exist. They could have been included under the location-based distributed protocols category as well, as they all assume that information about distances between nodes or relative positions is available.

The Relative Neighbor Graph eliminates the longest edge from every triangle formed by two of its neighbors and itself. The RNG can be easily determined using a local algorithm with message complexity $O(n)$ and computational complexity $O\left(n^{2}\right)$. Also, if the original graph $G$ is connected, then the reduced graph $G^{\prime}$ is also connected. However, nodes that are a few hops away in $G$ can become very far apart in $G^{\prime}$. The Gabriel Graph connects nodes $u$ and $v$ if the disk having line segment $u v$ as its diameter contains no other node than the two neighbors $u$ and $v$. The GG graph also maintains connectivity and has the same message and computational complexity of RNG.

As it can be seen RNGs and GGs are very similar; they both remove every link to a neighbor node that could be reached through another neighbor. Distributed implementations of the RNGs and GGs only require nodes to share their locations with their neighbors and test these conditions to verify each edge in order to determine the minimal set of neighbors. For example, a distributed version of the GG can be found in [15]. Although 
these two graphs have low message complexity $(O(n))$, their node degree can be as high as $n-1$.

Another graph borrowed from computational geometry that is useful to build reduced topologies is the Delaunay Triangulation. If all the neighbor nodes are connected based on the vicinity of the Voronoi diagram, a Delaunay Triangulation will be obtained. The Voronoi diagram is a geometric construction that defines the area of coverage and the vicinity in a graph. Trace an imaginary line between two nodes. Right in the middle of this line, trace an orthogonal line that will define the limit between the areas. Repeat the process between every pair of nodes in the graph. The smaller intersected area defined around a node from the limits with every other node in the graph determines the final diagram. The Delaunay Triangulation diagram is formed by connecting adjacent vertices in the Voronoi diagram. With this approach each node can choose as its transmission power the power needed to reach its farthest neighbor.

This approach requires global information and, if applied without restrictions, it may connect nodes which are more distant than the maximum communication range. DT has a message complexity of $O(n)$ and computational complexity of $O(n \log n)$. It also

guarantees connectivity and may have a node degree as high as $n-1$. In [16], a localized version of the Delaunay graph is described.

\subsubsection{Distributed Approaches}

Distributed topology construction protocols are described next. Here, the main concern of these algorithms is about building a "quality" topology while being able to implement it in an efficient fashion; where efficient means with low energy consumption, low computational and message complexity, and so on. These protocols make use of loca- 
tion, direction, neighbor, and routing information to achieve their objectives. Finally, the section also includes mechanisms that find the reduced topology by controlling the transmission power in heterogeneous networks, i.e., without assuming similarity of nodes in the network, which is in fact, a very plausible scenario.

The algorithms that solve the topology construction problem using location-based techniques assume that every node knows its own position with a high degree of certainty. This information allows them to use geometric properties in order to determine the best configuration of the topology in terms of distance between nodes, which at the end determines the best transmission range for each of them. For example, distributed versions of the algorithms from computational geometry described in Section 2.2.1.1 can be included here. Some of the most important location-based protocols are the Local Minimal Spanning Tree, or LMST [17], and the R\&M algorithm [18].

In direction-based techniques it is assumed that the nodes are able to determine the direction of the signals received from their neighbors, and in some cases, the distance between them. The direction of the incoming angle of the signal in the circular communication range can be provided by directional antennae installed in the nodes. Distance information, on the other hand, can be obtained using different techniques like Received Signal Strength Indicator (RSSI), Time of Arrival (TOA), Time Difference of Arrival (TDOA), or any other similar technique. More information about localization techniques can be found in [19-21]. Some of the most important direction-based protocols are the Yao Graph (YG) [22], the Cone-based Topology Control protocol (CBTC) [23], the Distributed Relative Neighbor Graph Protocol (DistRNG) [24] and the Angular Topology Control with Directional Antennas (Di-ATC) protocol [25]. In all the location- and direction-based techniques, the topology construction algorithms require extra information from the neighbor nodes other than their own presence, such as 
accurate Cartesian coordinate (bi- or tri-dimensional location) or polar coordinate (distance and angle). However, localization information is not always available or accurate, and it could be very expensive to obtain. For example, location from GPS-enabled nodes can only be obtained in places where there is direct access to the satellite signals. Other localization techniques, like ultrasonic or ultrawide band-based, not only need a localization protocol on top of the topology construction protocol, but could also increase the communications overhead, as their range is very small compared with the radio coverage. In the case of polar coordinates, the use of directional antennae increases the price and complexity of the wireless devices. In addition, each one of those techniques also carries an intrinsic error that limits the reliability on the information they produce. Neighborbased techniques overcome these problems, as they assume that nodes only need to have the ability to determine the number of neighbors, change their transmission power and, in some cases, calculate the distance between nodes.

The main idea of these algorithms is to produce a connected topology by connecting each node with the smallest necessary set of neighbors, and with the minimum transmission power possible. Given that the nodes do not posses accurate location information, their decisions depend mostly on the probability of selecting the appropriate neighbors, the ones that would extend the network as far as possible. Under the assumption that the nodes are either uniformly or Poisson distributed, some properties have been found in connected topologies that define a bounded minimum appropriate size of the neighborhoods of a single node that w.h.p. would create a connected topology. As a result, most neighbor-based protocols for topology construction are based on the creation of a $K$ neighbor graph.

The definition of the minimum number of neighbors $k$ that each node must have in order to preserve connectivity has been a well-studied problem. Most commonly used numbers 
for this parameter are between 6 and 8, or an average of 3 neighbors, as presented in [2628]. Recently, in [29], it is demonstrated that to produce a connected topology, each node should be connected to $\Theta(\log n)$ nearest neighbors. However, the only way to completely guarantee connectivity in a worst-case scenario, under the homogeneous assumption, is by defining $k=n-1$, which will produce a topology similar to the original MaxPower graph, assuming of course that it was connected from the beginning. Some of the most important neighbor-based protocols are the $K$-NEIGH protocol [30] and the XTC protocol [31].

The connectivity of a topology is one of the most important requirements of any topology construction protocol. One way to detect connectivity is by making sure that a route can be found from one node to every other node in the network. This is the main objective of the routing function: to build routing tables to route packets from one node to all possible destinations. When all the nodes are included in the routing tables it implies that they can be reached, and the transmission range does not need to be adjusted. This is the main idea behind the routing-based techniques. One of the most widely known topology construction mechanisms in this category is the Common Power (COMPOW) protocol [32].

\subsubsection{Heterogeneous Devices}

The wide spectrum of possible applications where wireless sensor networks can be applied has increased the possibility of mixed networks, where devices of different types and characteristics co-exist and work in the same application. In this type of heterogeneous environment, it is very important to devise algorithms and mechanisms that will allow different devices to collaborate, each taking advantage of the abilities of the others. 
Although topology control problems have been studied in the context of heterogeneous wireless sensor networks before, most existing mechanisms have focused on varying the nodes' transmission power based on the assumption that all the wireless devices have identical physical characteristics. As a result, topology control problems have been solved as range assignment problems, which not only neglect the heterogeneity of the network but also do not take advantage of the unique capabilities of different devices. Some examples of topology construction algorithms for heterogeneous wireless sensor networks are the Directed LMST (DLMST), Directed RNG (DRNG), proposed both in [33], and the Residual Energy Aware Dynamic (READ) topology construction algorithm [34].

\subsubsection{Hierarchical Techniques}

The previous section discusses how changing the transmission power of the nodes reduces the network topology, saves energy, and increases the lifetime of the network while preserving connectivity and coverage. However, this approach does not prevent the transmission of redundant information when several nodes are close to each other and may not simplify the network topology enough in order to make wireless sensor networks scalable for large deployments. This section explains a different approach to topology construction, the hierarchical topology construction approach, which addresses the scalability problem and facilitates the aggregation of information for additional energy savings. In the hierarchical topology construction approach, a communication hierarchy is created in which a reduced subset of the nodes is selected and given more responsibilities on behalf of a simplified and reduced functionality for the majority of the nodes. This approach has the potential to greatly simplify the network topology and the opportunity to 
save additional energy by assigning useful functions to the reduced subset of nodes, such as information aggregation and filtering, and routing and message forwarding.

One disadvantage of the hierarchical approach is that the selected subset of nodes will work more than their unselected neighbors, and will see their batteries drained sooner. Therefore, this approach must be accompanied by a good topology maintenance function that will rotate the role of the nodes with the final goal of spending their energy evenly and extending the network lifetime.

The classification of topology constructions mechanisms depicted in Figure 2.2 shows that hierarchical-based topology construction mechanisms can be classified as backbonebased, adaptive, and cluster-based. In the following sections, these categories along with the most important algorithms and protocols available in the literature are described and explained.

\subsubsection{Backbone-based Techniques}

The main goal of the communication backbone approach is to find a connected subset of nodes that will guarantee connectivity by allowing every other node in the network to reach at least one node on the backbone in a direct way. It is important to mention that even though one common assumption of this approach is to have the nodes transmitting at full power, they could also reduce their transmission range to reach only their direct active neighbors, so the approaches presented in Section 2.2.1 and this one are not mutually exclusive. Figure 2.3(b) shows an example of this approach in which only 35 nodes out of 500 nodes in the network were selected to build the backbone and to provide complete connectivity. 


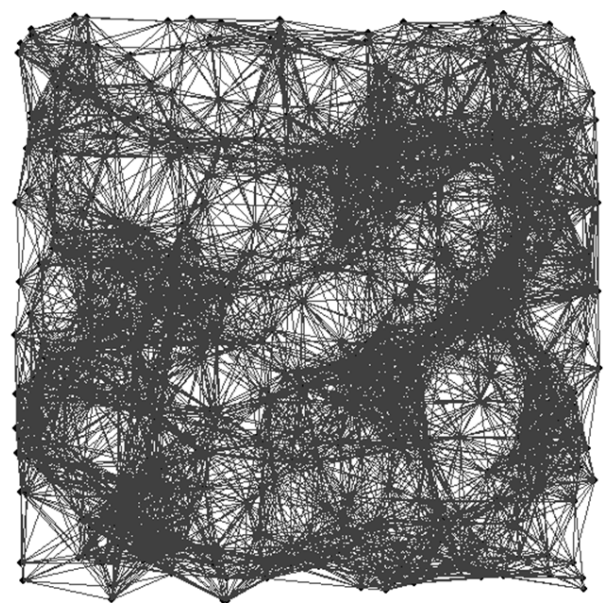

(a) MaxPower graph.

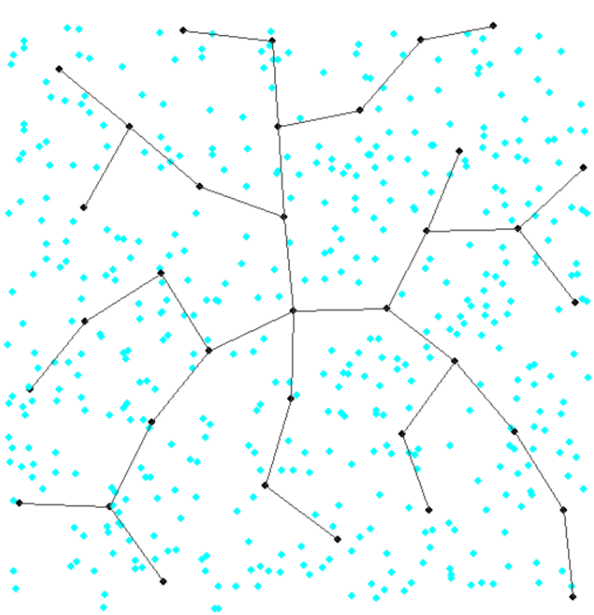

(b) Reduced topology.

Figure 2.3: Topology control by reducing the number of active nodes and the creation of a network backbone.

A communication backbone can be created by solving a widely known mathematical problem: the Connected Dominating Set $(C D S)$ problem. A Dominating Set $(D S)$ is a set of nodes $D \subset V$ in a graph, in which all other nodes that do not belong to the subset have a link to at least one node in the set $D$. In the special case of the CDS, the nodes in $D$ are connected. Mathematically, the formal definition of a DS is expressed as in Equation 2.4. Of course, the smaller the dominating set is, the better for energy conservation, therefore, finding the Minimum Dominating Set (MDS) and the Minimum Connected Dominating Set $(M C D S)$ is of paramount interest. These problems have been shown to be NP-hard in $[35,36]$, therefore, heuristics are needed for practical purposes.

$$
D=(\forall v \in V: v \in D \vee \exists d \in D:(v, d) \in E)
$$

Some mathematical formulations of the problem has been proposed for the MCDS problem. In [37], the authors propose the separation of the problems of the CDS into two parts to which well-known linear programming definitions exist: the minimum dominating set and the minimum tree that will connect the dominating nodes. Some other variations have 
been proposed, adding restrictions to the problem in order to reduce the solution space: in $[38,39]$ where the authors restricted the type of networks to be regular and random cubic graphs, respectively. Other solutions extend the CDS scheme by including other metrics, like in [40] where the authors include the maximization of the throughput of the network. In this dissertation, a new mixed integer programming definition of the MCDS problem is defined in Section 3.2 as a new tool for evaluation of the protocols proposed in this dissertation.

In the case of approximate solutions to the MCDS problem, many algorithms have been proved to be approximation algorithms of the optimal solution. In the work of Guha and Kuller [41], the authors proposed two schemes to approximate the MCDS with ratios of $O(H(\Delta))$, where $H(\cdot)$ is the harmonic function and $\Delta$ is the maximum node degree of the graph. However, assuming that the nodes are deployed in the Euclidean space, better approximation ratios can be achieved. For example, the algorithms based on Maximal Independent Sets (MIS) presented in $[42,43]$ and $[44,45]$ have been proved to have an approximation ratio of $O(n)$, and 7.8 and 6.91 to the size of the optimal MCDS, respectively.

In general, three methods are the most commonly used to create a connected dominating set, and they will be explained in detail. These techniques are: "growing a tree", connecting independent sets, and pruning-based techniques.

An easy way to illustrate the first approach to "grow a tree" is by comparing it with Prim's algorithm [46], which finds the minimum spanning tree of a graph. The process works from the set of nodes that are part of the tree at time $t$. Between $t$ and $t+1$, based on certain parameters, the nodes in the set evaluate all adjacent nodes in order to extend the tree. The process continues until all nodes on the graph are evaluated. Of course, in the case of the CDS, not every node will be selected to be part of the tree, and those which 

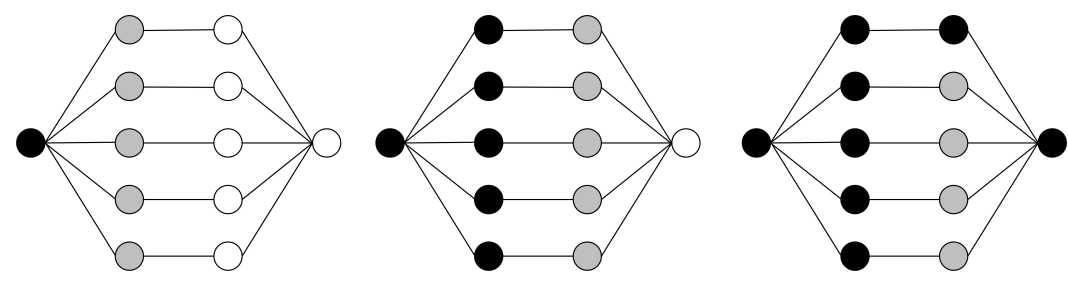

Figure 2.4: Growing a tree with 1-hop neighbor information.

were not selected will go to sleep until a new tree is requested. This procedure guarantees that the extended tree at $t+1$ is still connected since the new selected nodes are always neighbors of at least one node of the tree set. In general, the process starts on a single node, usually the sink node, but if there are more than one sink nodes, then several trees could be built in parallel, if the nodes support that functionality. The "growing a tree" technique has characteristics that are desirable for routing protocols, like an organized structure that allows implementing routing based on the simplified logical structure, hierarchical addressing, querying based on tree searches, etc.

In [41], the authors present a coloring-based growing a tree approach to create a CDS on a graph. At the beginning, all nodes are marked as White, and the algorithm starts at the node with the highest node degree. This node is marked Black, and marks all its neighbors Gray. Gray nodes are inspected in order to calculate their "yield", or the number of White nodes that they would add to the tree. The Gray nodes that include more unmarked nodes on each iteration will be included on the tree by marking them as Black nodes. However, using the topology depicted in Figure 2.4, the authors showed that this approach is ineffective if Gray nodes looked at their own neighbors only. As it can be seen, the algorithm creates a tree with $d+2$ nodes, where $d$ is the node degree of the initial node, instead of the expected tree with only four nodes. The authors also show that the implementation of this algorithm runs in $O(m)$ steps, where $m$ is the number of edges in the original graph. 
In order to solve this problem, the authors proposed a modification of the algorithm, consisting of scanning pairs of nodes in a 2-hop manner. A Gray node and a White node will be marked as Black if their joint contribution is the greatest on the iteration. First, the algorithm marks a Gray node Black, which makes all its neighbors Gray. Then, one of the Gray nodes is also colored Black, which makes its neighbors Gray. Here, the yield is given by the total number of Gray colored nodes. Finally, the pair of nodes with the highest yield is the one selected as part of the tree. This "look ahead" greedy solution not only produces the expected four-node tree but also let the authors prove that the procedure produces a connected dominating set with at most $2(1+H(\Delta)) \times\left|O P T_{D S}\right|$ nodes, where $\Delta$ is the maximum degree of the graph, $H(\cdot)$ is the harmonic function $H(k)=\sum_{i=1}^{k} 1 / i \leq$ $\ln k+1$, and $\left|O P T_{D S}\right|$ is the set of nodes in an optimal dominating set. The authors show that the implementation of this modified greedy algorithm can be run in $O(\mathrm{~nm})$ steps, where $n$ is the set of nodes and $m$ the set of edges in the original graph. A distributed version of this technique is presented in [47] with an approximation of at most $2 H(\Delta)$ nodes than the optimal solution, and $O(|C|(\Delta+|C|))$ time and $O(n|C|)$ message complexity, where $C$ is the dominating set produced by the algorithm.

The topology construction protocols introduced in this dissertation, the A3 family of protocols, use the "growing tree" technique to build a connected dominating set. A detailed explanation of the protocols will be presented in Chapters 4 and 5 .

The second approach to building a CDS tree is to create independent sets first, and connect the sets later. During the first phase, the idea is to form a Maximal Independent Set (MIS). An Independent Set (IS) of graph $G$ is a subset $W \in V$ where no two nodes in $W$ have an edge. An MIS is an independent set that is not a subset of any other independent set, i.e., an MIS of $G$ is an IS that cannot include more nodes in $V$. Therefore, an MIS is a Dominating Set, which may not be connected. During the second phase, these algorithms 
find a way to connect the DS with the minimum number of nodes, and therefore, form the CDS tree.

The Energy Efficient Connected Dominating Set (EECDS) algorithm is an important example in this category. Proposed in [44], the EECDS algorithm creates a maximal independent set in the first phase, and then selects gateway nodes to connect the the independent sets during the second phase.

EECDS also uses a coloring approach to build the MIS. The EECDS algorithm begins with all nodes being White. An initiator node elects itself as part of the MIS coloring itself Black and sending a Black message to announce its neighbors that it is part of the MIS. Upon receiving this message, each White neighbor colors itself as Gray and sends a Gray message to notify its own White neighbors that it has been converted to Gray. Therefore, all White nodes receiving a Gray message are neighbors of a node that does not belong to the MIS. These nodes need to compete to become Black nodes. The competition consists of sending an Inquiry message to its neighbors to know about their state and weights and wait for their responses for a specific amount of time. If during this time, it does not receive any Black message in response, and it has the highest weight, it becomes a Black node, and the process starts again. Otherwise, it stays as a White node. The weight is a metric calculated by each node based on the battery power and effective node degree. The authors show that the set of Black nodes produced by the above algorithm forms a MIS.

The goal of the second part of the algorithm is to form a CDS using nodes that do not belong to the MIS. These nodes, called connectors, are selected in a greedy manner by MIS nodes using three types of messages. A non-MIS node that becomes part of the CDS sends a Blue message to notify its neighbors. MIS nodes send Invite messages to non-MIS nodes to invite them to be connectors. In response to invite messages, non-MIS 
calculate their weights and send Update messages. Finally, the non-MIS node with the highest weight becomes part of the CDS. The authors prove that phase two of EECDS builds a CDS.

The main disadvantage of the EECDS algorithm is its message complexity. In both phases of the algorithm, competition is used to determine the best candidates to be included in the independent sets and the final tree. This process is very costly in terms of message overhead because each node has to consult its neighbors for their status in order to calculate its own metric. This large overhead is particularly detrimental in dense networks because of the network congestion and collisions that it generates. The authors claim that the message complexity of the EECDS algorithm is $O(n)$, as during each of the two phases each node at most sends out one message. The time complexity of the algorithm is given by the construction of the MIS, which has a $O(n)$ worst time complexity. The authors also show that EECDS has an approximation factor of not higher than 7.6, i.e., the EECDS produces a CDS with at most 7.6 times the number of nodes given by the optimal solution, which is the MCDS. Finally, the authors propose a backbone recalculation procedure to switch the backbone when the minimal energy of the current one decreases by $50 \%$. This procedure, unfortunately, is not well-explained in the paper and it is unkonwn whether it needs global information or if it can be triggered in a distributed manner. Further, the claim that the recalculation procedure balances the energy consumption of the nodes is not supported.

Other algorithms that work using this technique are the Distance-2 (D2) Coloring algorithm [48] and SPAN [49]. A modified version of the SPAN algorithm that includes the use of directional antennas and presents the same message and computational complexity of the original version is presented in [50]. Other algorithms that belong to this category can be found in [51], and [41]. 
Finally, the third approach to create a CDS works differently that the two others approaches described above; the growing tree and the independent sets-based techniques start with a reduced, non-connected topology and then add nodes to connect it. In the Pruningbased techniques approach, the algorithms calculate a topology that guarantees connectivity by including most of the nodes, and then pruning unnecessary nodes out. This approach is used in the Connected Dominating Set under the Rule K algorithm proposed in $[52,53]$ and the Extended Connected Dominating sets introduced in [54].

The Connected Dominating Set under Rule K (CDS-Rule-K) algorithm proposed in [52, 53 ] is an example of a distributed algorithm under this category that uses local information. The algorithm works in two phases. The first phase involves creating an initial CDS tree using the following marking process [52]:

$$
S=\left(\forall v \in S: x, y \in N_{v}, \neg \exists(x, y) \in E\right)
$$

where $N_{v}$ is the set of neighbors of $v$.

In other words, if node $v$ has two neighbors that are not connected, it will include itself on the initial set. In this first phase, the nodes exchange HELLO messages in order to get to know their neighbors and exchange their neighbor lists. Once a node receives the lists from its neighbors, it intersects the lists with its own list of neighbors and counts the number of elements in both lists. If this number is less than the amount of neighbors in the node's own list, then this node will mark itself as part of the initial set; otherwise, the node will turn itself off.

For the second phase, the algorithm prunes unnecessary nodes applying one of the three pruning rules described in [53]. A temporarily marked node decides to unmark itself if it determines that all its neighbors are covered by marked nodes with higher priority, which might be given by the level of the node in the tree assuming that a lower level means 
higher priority, or the priority could have been defined before as to the execution of the protocol. An initiator node requests the list of all marked nodes in its neighborhood with less or equal priority than itself. The neighbor nodes update their list of marked nodes and respond with the updated list. Then, based on the reply messages, the initiator checks if all its neighbors are covered by at least one active node different from itself. If this is the case, it unmarks itself and announces this, so the other nodes can update their status of covered neighbors. If the node has at least one node that is covered exclusively by itself, then it marks itself as a permanent element of the tree. The final tree is a pruned version of the initial one with all redundant nodes that were covered by other nodes with higher or equal priority removed.

Other pruning rules are also presented by the authors in [52, 53]: Rule 1 and Rule 2. In Rule 1, a marked host can unmark itself if its neighbor set is covered by another marked host; that is, if all neighbors of a gateway are connected with each other via another gateway, it can relinquish its responsibility as a gateway. In the Rule 2 pruning approach, a marked host can unmark itself if its neighborhood is covered by two other directly connected marked hosts. Finally, the third and last rule, Rule $K$, generalizes the approach and builds a better (smaller) CDS relaxing the constraint on the number of marked hosts, i.e., Rule $K$ unmarks gateways covered by $k$ other gateways, where $k$ can be any number. The cases of the querying of neighbor node's status and the unmarking announcement processes in the EECDS algorithm and the CDS-Rule-K mechanisms respectively, show the main source of messages complexity in those protocols. Even though the authors claim linearity in the message complexity, the final amount of messages is not bounded and is affected by node degree and node density in the network. In the CDS-Rule-K protocol, once a node decides to unmark itself during the query process, each node generates a query that is based on its own level and receives individual answers. During the 
unmarking announcement process, it must update all its neighbors to correct the metrics in all possible active processes. This is two-hop information that most of the nodes send once they get pruned from the tree. The CDS-Rule-K mechanisms has an $O\left(n^{2}\right)$ message complexity, $O\left(n^{2}\right)$ computational complexity, and an $O(1) \cdot D S_{o p t}$ approximation.

\subsubsection{Cluster-based Techniques}

This section presents cluster-based techniques, which simplify the network topology even further by creating groups of nodes managed by special nodes called Clusterheads. Clusters are created by classifying objects into different groups, or more precisely, partitioning the data set into subsets so that each cluster shares some common feature, characteristic, or trait. Cluster-based techniques also include algorithms to select the clusterheads, special nodes that not only serve as facilitators for communication on behalf of their cluster nodes but also can perform special tasks such as routing, data aggregation, scheduling, and others with the potential to save even more energy.

Both clustering techniques and clusterhead selection algorithms are very important in these topology construction techniques since they have to be implemented in a distributed fashion, consume the network energy evenly and efficiently, and introduce the least amount of extra overhead. Some of the more important algorithms of this kind are the Low Energy Adaptive Clustering Hierarchy (LEACH) protocol [55], which is one of the most widely known cluster-based techniques, and Hybrid Energy-Efficient Distributed (HEED) clustering, a hybrid, energy-efficient, distributed clustering approach for ad hoc sensor networks [56]. 


\subsubsection{Adaptive Techniques}

Adaptive techniques are those mechanisms in which the network is periodically evaluating the state of the communication, and, when a performance metric is not at its desired level, the reduced topology adjusts in order to recover the normal state. Adaptive techniques for topology construction in wireless sensor networks are not very common in the literature thus far. One interesting feature of these techniques is that they perform both, topology construction and topology maintenance functions. Some of the most important adaptive protocols are the Adaptive Self-Configuring sEnsor Networks Topologies (ASCENT) mechanism [57], and the Minimum Power Configuration Protocol (MPCP) and the Minimum Active Subnet Protocol (MASP) both proposed in [58].

\subsubsection{Hybrid Approaches}

The techniques presented in previous sections can be combined in order to develop a more complete and better solution to the topology construction problem. For example, clustering techniques might be used along with controlling the transmission power of each node to simplify the topology and solve the Range Assignment problem, saving additional energy.

An example of a hybrid technique is CLUSTERPOW [59], in which the authors combine clustering, router-based, and power control techniques to solve the RA problem in networks with non-homogeneous deployments. Other examples of hybrid protocol are the Topology Management by Priority Ordering (TMPO) protocol [60], the Topology and Energy Control Algorithm (TECA) for Wireless Sensor Networks [61], and the protocols proposed in [62, 63]. 


\subsection{Coverage-oriented Topology Construction}

It has been stated in this dissertation that the main goals of a wireless sensor network are to monitor the occurrence of events in the area of interest, report those events to the users, and be active the longest possible time while covering as much area as possible and preserving connectivity, so the information generated from the nodes can be collected. This means that in order to have an effective topology construction protocol, none of these issues should be ignored. The previous section included multiple topology construction protocols that provided a connected reduced topology, but did not take into account the level of coverage of the area of interest. This section presents topology construction protocols whose mail goal is to guarantee coverage. In order to preserve generality and given that some of the protocols included in this taxonomy do not guarantee connectivity in the network, the proper name of this branch could not have been connected-coverageoriented, even though the contributions in this area presented in this dissertation do guarantee connectivity.

Looking at the already presented protocols, two cases of reduced topologies comes to attention: The first one is case where all the nodes are kept awake and just their communication ranges are reduced, and the second one is where the topology is reduced by turning off redundant nodes. In the first case the coverage of the area depends exclusively on the actual deployment of the nodes because the coverage of the reduced topology remains exactly the same as in the MaxPower graph. In the second case, each node that is turned off does most likely reduce the degree of coverage on the area of interest, which implies that the selected nodes must include some metric to minimize the impact of the sleeping nodes while guaranteeing a high degree of coverage. 


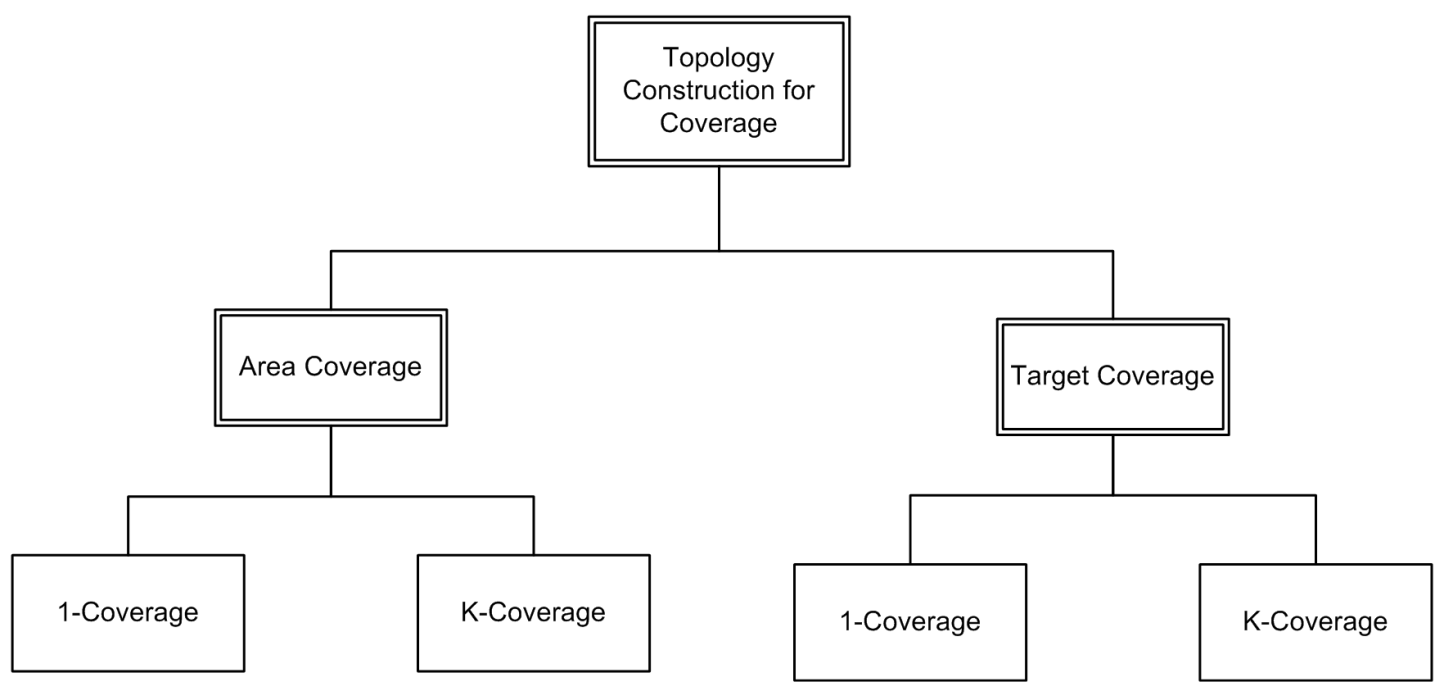

Figure 2.5: Classification of coverage-oriented topology construction protocols.

The goals of total coverage and energy efficiency are tightly integrated since a network that provides total coverage might have too many active nodes, a topology that is simply connected might not provide sufficient coverage of the deployment area, or an optimal total coverage with minimum number of active nodes may not be connected. Depending on the application, any of these cases may be the desired solution. This section includes a series of classification criteria that serve as a structure for a general taxonomy of coverageoriented topology construction protocols. Following the taxonomy and its classification criteria, some of the most important coverage-oriented protocols are introduced.

\subsubsection{Classification Factors}

Coverage-oriented topology construction algorithms can be classified according to the definition of the area of interest and the redundancy they provide. The topology construction protocols included in this chapter are organized based on their classification in the taxonomy, as shown in Figure 2.5. 


\subsubsection{Definition of the Area of Interest}

The definition of the area of interest is the main factor that differentiates coverage-oriented topology construction protocols. Depending on the application and the events to be monitored, the specification for coverage changes.

The most general case is the Area Coverage, in which the wireless sensor network is in charge of monitoring the complete area for the occurrence of events. Some typical examples of these applications are intrusion detection and mapping of environmental variables, like temperature. The main issue with the problem of complete coverage is that, if its a very strict policy, it may require a large amount of active nodes, especially if the density of the network is low. In addition to this, in order to have a precise idea of the covered area of a node, the position of the nodes is usually required and this increases the cost of implementation due to special hardware (like GPS) or overhead if a distributed location algorithm is used.

However, sometimes it is not necessary to monitor the entire area but a set of smaller regions in which the real interest is. In some cases it may be assumed that these areas of interest may even change in time. The protocols in this category are known as Target Coverage protocols. Their main interest is to optimize the coverage of a certain number of targets inside the deployment area while possibly reserving the energy in areas in which there is no interest in keeping them covered because they do not contain any targets. An application that benefits from the protocols in this category is target tracking. One of the issues of this kind of coverage is how to guarantee coverage in the cases where the mobility of the targets require the protocol to change the selection of active nodes frequently. 


\subsubsection{Redundancy}

Given the promptness for failure in wireless sensor networks, coverage can be affected in a very unexpected way when a node fails. Some applications can withstand a certain degree of failure in coverage and wait for a maintenance policy to restore it later. In other applications the loss of coverage is not an option and some level of robustness must be guaranteed in order to withstand node failure. One of the ways of procuring robustness is by increasing redundancy; i.e., selecting more than one node to cover the same area. The most common case of redundancy is 1-coverage, in which the protocols just guarantee that at least a single node is covering each point of the area. This is the solution that requires fewer resources from the network, but is also the one that is more sensitive to node failure because it can leave areas uncovered with the loss of a single node. Non critical applications benefit from protocols in this category because they can reserve more energy for future use and extend the lifetime of the network.

The protocols that offer a higher degree redundancy are in the category of $K$-coverage, where $\mathrm{K}$ is the number of nodes that are guaranteed to offer coverage over each point in the interest area. Applications that need complete coverage find the K-coverage algorithms fitted so they can still offer coverage even when K-1 nodes fail. The main issue of these protocols is that the amount of resources needed to offer redundant coverage can be very high, and also may have implications concerning the density of active nodes in the reduced topology. 


\subsubsection{Other Considerations in Coverage-oriented Topology Construction Protocols}

Some of the protocols in the coverage-oriented category have special considerations related to the communication and sensing ranges of the nodes. Similarly to topology construction for connectivity, both communication and sensing ranges are usually represented as disks centered at the node's position with radius $R_{\text {Comm }}$ and $R_{\text {Sense }}$, respectively. One of the most common assumptions in the literature is that, in order to facilitate the implementation of a solution that guarantees both coverage and connectivity, the communication range is set to be $R_{\text {Comm }} \geq 2 \cdot R_{\text {Sense }}$. In the works presented in [64-66], the authors proved that if the communication range is at least twice the sensing range, a complete coverage of the area will imply connectivity among the active nodes in the topology. This work also was extended to K-coverage in [67].

Some other protocols work under the assumption that the the communication and sensing ranges are equal [68-70]; however, in reality communication range is usually greater than the sensing range and, even if the communication range could be reduced to match the sensing one, it would imply a substantial increase in the number of nodes required to cover a given area. In both cases, the protocols that use these assumptions cannot guarantee connectivity if the assumption is not accomplished. To solve this dependency, many protocols assume an arbitrary ratio between the communication and sensing ranges, including the ones introduced in this dissertation, A3Cov and A3CovLite. Another consideration regarding the coverage-oriented protocols is whether they need location information or not. The main advantage of having location information is that it is easier to guarantee total coverage because each node can calculate the area covered by itself and its neighbors, check redundancy and determine whether it is needed to stay active or not. However, even if the cost of the overhead is not considered, the accuracy of 
the location information may not be enough to guarantee the precision in the calculation of the coverage; in other words, due to an incorrect location, a node may leave a zone uncovered assuming the area is covered by its neighbors.

In the case of the protocols that lack any location information from the nodes in the network, guaranteeing coverage is a more complex task which usually is solved by providing an approximate solution. One of these approximations is presented in [71], which states that in a dense network, if all the nodes in the network are covered by the sensing range of at least one active node, then the area of deployment is covered w.h.p. This approximation may imply a sacrifice in coverage area, but will also decrease the number of active nodes required to offer a high level of coverage.

\subsubsection{Examples of Coverage-oriented Topology Construction Protocols}

The first part of this section will be dedicated to Area Coverage protocols, presenting both 1-coverage and K-coverage protocols. Different approaches will be presented, including theoretical deployments, and centralized and distributed solutions. The second part of this section will cover some of the most relevant target coverage protocols, including also different levels of redundancy.

The first and most common case of coverage-oriented protocols is the area coverage category. The first case that will be considered will be the protocols that provide 1-coverage of the area of interest. Some theoretical approaches generate optimal grid deployments that guarantee connected coverage with a minimum number of nodes, and different levels of connectivity. Some examples of this theoretical approach are presented in $[69,72-$ 75]. Furthermore, the solution to the circle packing problem is also considered as an approximation to the connected coverage problem, based on the results in [76], assuming 
that $R_{\text {Comm }}=2 \cdot R_{\text {Sense }}$ and that overlapping of the areas of the circles is possible. In this dissertation, the A3, A3Lite, A3Cov and A3CovLite protocols are compared with the optimal solutions presented in $[69,72,76]$.

Based on the assumptions of very dense networks and a communications radius equal to the sensing radius $\left(R_{\text {Comm }}=R_{\text {Sense }}\right.$ ), the authors in [69] proved that no topology can guarantee connected-coverage to the entire area with a node density lower than $\left(0.522 / r^{2}\right)$, where $r$ is the communication and the sensing radius.

In addition, the authors use specific geometries to create grid-like topologies of active nodes to provide connected-coverage of the deployment area. The grid structures are based in square-, hexagon-, and strip-like distributions. In order to calculate the amount of nodes needed to build this topologies, the authors calculate the node density of each type of grid. This formulas are presented in Equations 2.6.

$$
\begin{aligned}
d_{S Q R} & =1 / r^{2} \\
d_{H E X} & =0.769 / r^{2} \\
d_{S T R} & =0.536 / r^{2}
\end{aligned}
$$

which have approximation ratios of $1.916,1.473$ and 1.027 with respect to the optimal connected-coverage topology, and a degree of connectivity of 4, 3 and 1, respectively. The authors also present approximation schemes to implement these theoretical deployments in distributed fashion. These distributed algorithms are all based on the assumption of very high dense network deployments, the need of location information and a reference location system, and message exchanges among neighbors to make on/off decisions without further coordination. 


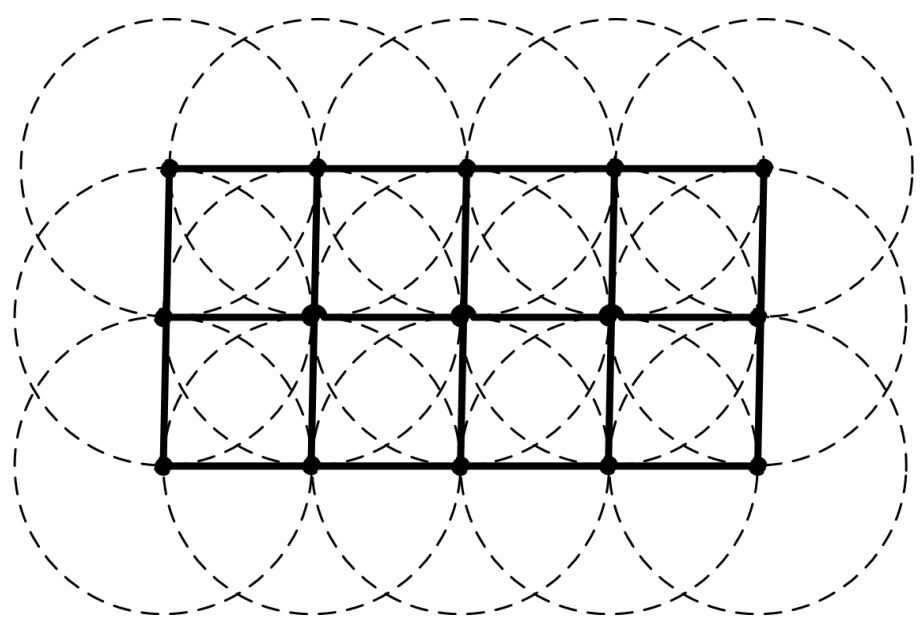

(a) Square deployment.

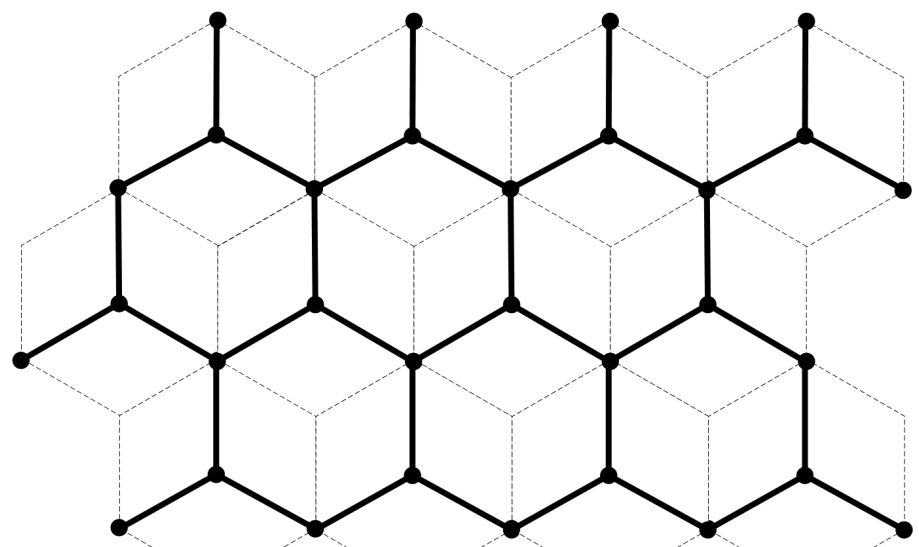

(b) Hexagon deployment.

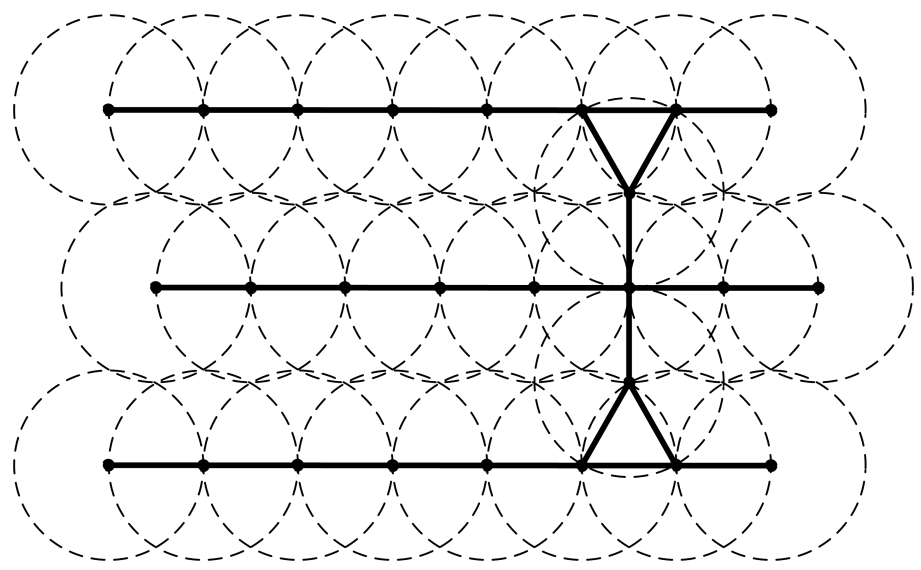

(c) Strip deployment.

Figure 2.6: Different deployment geometries for connected coverage topologies with $R_{S}=R_{C}$. 
Another interesting result shown in [69] is that moderate node densities can be used to cover a large fraction of the area whereas the required density of nodes increases drastically if complete coverage is needed. Based on this result, simulation experiments are presented to provide $90 \%$ connected-coverage topologies only. However, the auhtors do not provide details of the distributed implementations.

Similar optimal deployments are also presented in [72] where the authors work with squares, triangles, rhombuses, hexagons, and strips but prove optimal deployments for scenarios where $R_{\text {Comm }} \neq R_{\text {Sense }}$, with node density functions presented in Equations 2.7. As shown in [72], once $R_{\text {Comm }}>\sqrt{3} R_{\text {Sense }}$, most optimal topologies reach their minimal deployment size. This can be explained by the work in [77], where the author proved that the optimal coverage structure is based on a triangular pattern in which three circles with sensing radius radius $r$ are $\sqrt{3} r$ apart from each other. In other words, smaller distances between circles would produce overlapping regions while greater distances would produce non-covered areas. The repetition of this pattern creates a "honeycomblike" structure that provides total coverage with minimal number of circles. This factor determines that the distance between nodes to provide total connectivity-coverage should be $\min \left\{\sqrt{3} R_{\text {Sense }}, R_{\text {Comm }}\right\}$. On the other hand, if the ratio is $R_{\text {Comm }} / R_{\text {Sense }}=1$, the optimal sensing coverage is equivalent to communication coverage, which at the end determines the connectivity of the network. Based on these ideas, the experiments in the performance evaluation of the distributed algorithms use the ratio $R_{\text {Comm }} / R_{\text {Sense }}=\sqrt{3}$ for the connected-coverage protocols, and the ratio $R_{\text {Comm }} / R_{\text {Sense }}=1$ for the connectivityonly protocols. 


$$
\begin{aligned}
d_{S Q R}= & R_{\text {Sense }}^{2}\left(\min \left\{\sqrt{2}, \frac{R_{\text {Comm }}}{R_{\text {Sense }}}\right\}\right)^{2} \\
d_{R H O}= & R_{\text {Comm }}^{2} \sin (\Theta) \\
& \frac{\pi}{3} \leq \Theta \leq \frac{\pi}{2} \text { and } \sqrt{2} \leq \frac{R_{\text {Comm }}}{R_{\text {Sense }}} \leq \sqrt{3} \\
d_{H E X}= & \frac{3}{4} \sqrt{3} R_{\text {Sense }}^{2}\left(\min \left\{1, \frac{R_{\text {Comm }}}{R_{\text {Sense }}}\right\}\right)^{2} \\
d_{\text {STR }}= & 1 / \beta \sqrt{R_{\text {Sense }}^{2}+\beta^{2} / 4} \\
\beta= & \min \left\{R_{\text {Comm }}, \sqrt{3} R_{\text {Sense }}\right\}
\end{aligned}
$$

After observing the density equations from the previous solutions, if $R_{\text {Comm }}>>R_{\text {Sense }}$, the sensing range ultimately determines the number of active nodes to cover the complete area. In addition, it was mentioned that an approximation ratio of the strip-based deployment is no greater that $2.7 \%$ of the optimal solution, which is definitely a tight bound. In this dissertation, a new lower bound metric is proposed in order to support the performance analysis of coverage-oriented protocols: the Circle Packing Problem (CPP). The packing problem is very well-known by mathematicians and geometricians. One of the most important references to this problem can be dated back to 1900 when David Hilbert presented at the International Mathematical Congress, a list of 23 problems that would be relevant in the 20th century. Among the problems Hilbert presented, the problem of packing was one of those. The following quote was taken from his lecture[78] ${ }^{2}$ :

How can we arrange most densely in space an infinite number of equal solids of given form, e.g. spheres with given radii [...] that is, how can one

\footnotetext{
${ }^{2} \mathrm{~A}$ copy of Hilbert's complete lecture can be found at http://aleph0.clarku.edu/d̃joyce/hilbert/problems.html
} 
so fit them together that the ratio of the filled to the unfilled space may be as great as possible?

The solution of the packing problem, or more specifically the Circle Packing Problem, provides the minimum number of non-overlapping circles that can be fitted inside a geometric shape, in the case of study in this work, a unit square. The solution to this problem therefore provides a lower bound for total coverage based on the fact that none of the circles overlap each other, which limits the number of circles to a certain optimal value. The optimal solutions to this problem will be used in this dissertation for evaluation purposes. The optimal values provided by the circle packing problem [76] will be used as the lower bound for the connected-coverage problem. In addition, a modification is being proposed in order to generate total area coverage based on the results of the non-overlapping version: Let $R_{\text {Comm }}>>R_{\text {Sense }}$ so that the graph is connected. Let $n$ be the number of circles of a solution to the packing problem for radius $r=R_{\text {Sense }}$. If the sensing radius of the same circles is multiplied by a factor $c$ and overlapping is allowed, the unit square could become completely covered.

An example of this can be seen in Figure 2.7, in which the white sections are the original covered areas, the black sections are areas not being covered, and the outer circles represent the modified sensing area $c \cdot R_{\text {Sense }}$. Figure $2.7($ a) shows the optimal original circle packing for $R_{\text {Sense }}=1 / 6$ of the area side. Figures $2.7(\mathrm{~b}), 2.7(\mathrm{c})$ and $2.7(\mathrm{~d})$ show how the coverage would look if the radius is augmented by a factor of $2, \sqrt{3}$ and 1.5 , respectively. As it can be seen, the unit square is now completely covered in all scenarios, and the optimal value for packing can be compared with the ones obtained using the density functions of the optimal deployments with radii of $2 r, \sqrt{3} r$, and $1.5 r$. Figure 2.8 shows this comparison where it can be seen that the solutions to the packing problem with $2 R_{\text {Sense }}$, $\sqrt{3} R_{\text {Sense }}$ and $1.5 R_{\text {Sense }}$ are equivalent to the square, hexagon and strip geometric optimal 


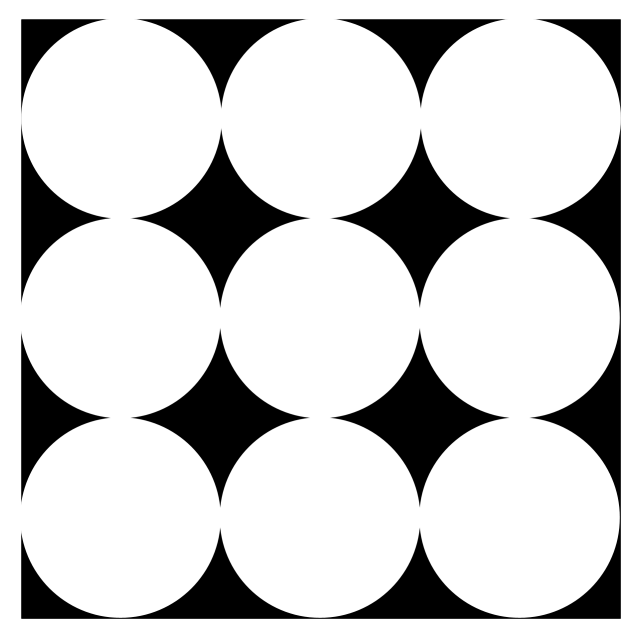

(a) Original circle packing with $R_{\text {Sense }}=$ $1 / 6$ of the area side.

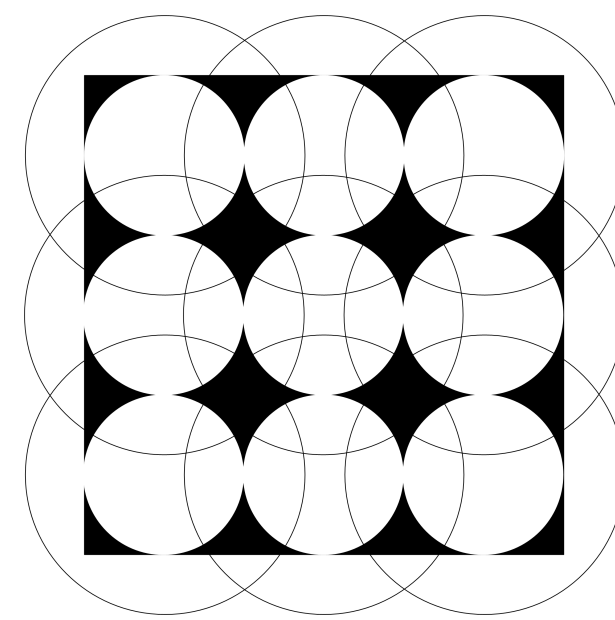

(c) Modified circle packing with $\sqrt{3} R_{\text {Sense }}$.

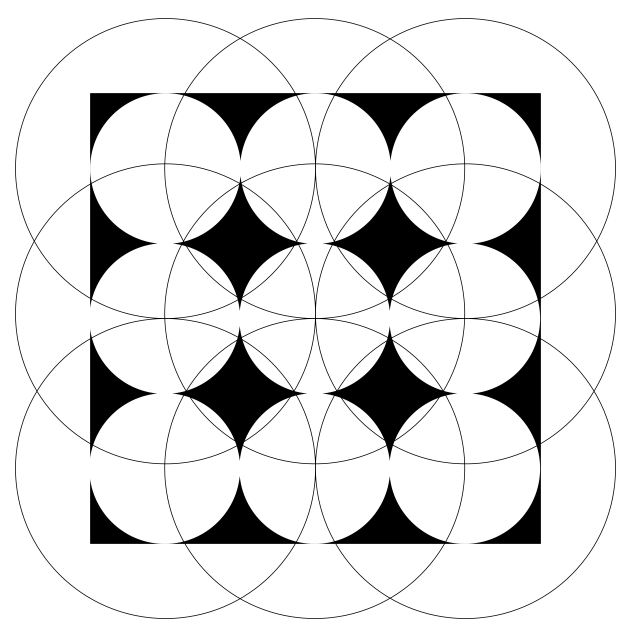

(b) Modified circle packing with $2 R_{\text {Sense }}$.

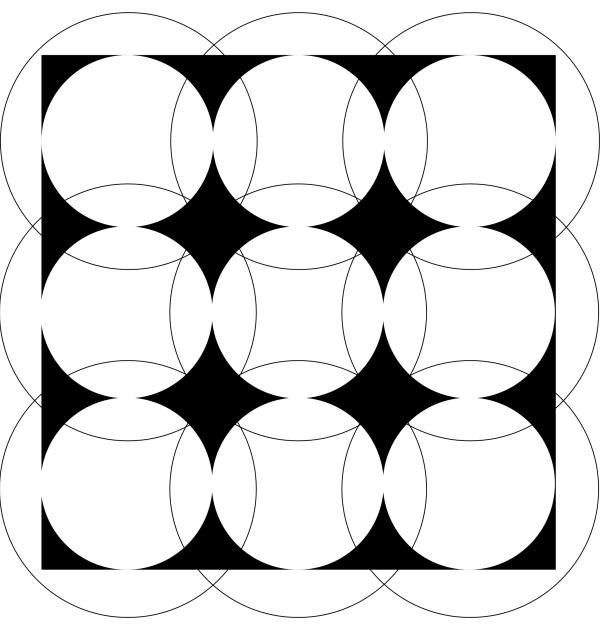

(d) Modified circle packing with $1.5 R_{\text {Sense }}$.

Figure 2.7: Example of original and modified solutions of the circle packing problem. 


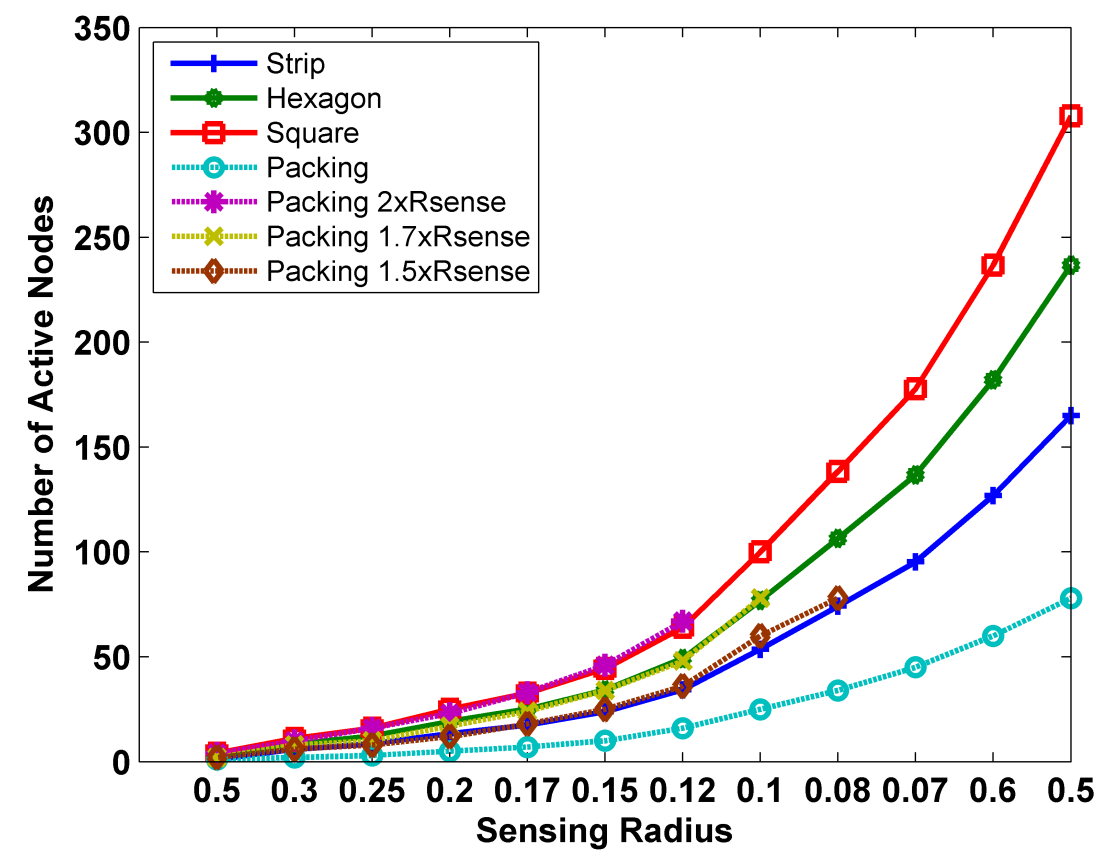

Figure 2.8: Theoretical comparison between the packing problem and the optimal deployments.

deployments presented in [69], in terms of number of active nodes, which validates the use of the solutions of the CPP problem as a tool for analysis.

The main issue with the optimal deployments is that the authors assume the possibility of manual location of the nodes in the exact pattern generated by the solutions. When the topologies have been deployed randomly, the optimal solutions cannot be applied directly. In order to solve the connected coverage problem in random topologies, the following centralized and distributed solutions have been proposed.

Some examples of centralized algorithms are the following: the authors in $[79,80]$ fit a random node deployment to a predefined grid. The authors in $[79,80]$ utilized the Triangle Lattice deployment pattern in order to find the lower bound for the coverage problem. The drawback is that although the tight allocation of circles in the area provides an almost complete coverage of the area, which is a lower bound for the total coverage, it results 
in an unconnected network. In order to solve this problem, the solution to the packing problem is used here to create a deployment that assures a minimum amount of nodes with $R_{\text {Comm }}=2 R_{\text {Sense }}$.

In [81], the authors grow a tree and add nodes according to the rate of new information that they provide about the monitored area. In the case of $[82,83]$, both solutions are based on metaheuristics. While the first work uses genetic algorithms to find a connectedcoverage topology that will cover a set of critical points, the second uses an evolutionary multiobjective approach to create an optimal deployment considering connectivity, coverage and transmission power.

Some examples of 1-coverage distributed protocol are the Proving Environment and Adaptive Sleeping (PEAS) protocol, Optimal Geographical Density Control (OGDC) protocol and the Area-based Collaborative Sleeping (ACOS) protocol. The PEAS protocol, presented in [84], offers coverage and connectivity asymptotically almost surely (a.a.s) in the cases where $R_{c} \geq(1+\sqrt{5}) R_{s}$. It works based on two algorithms: Probing Environment and Adaptive Sleeping. In the first one, nodes wake up from a sleeping period and probe their environment; if no node is active on a predefined probing range, then the node will wake up, otherwise it will go back to sleep. Adaptive Sleeping is used to calculate of the optimal wakeup time for the nodes. This protocol is a distributed heuristic to cover area, based on 1-hop away information from neighbors, and does not guarantee neither 1or k- connectivity or coverage. However, it does not require location information and it does provides a maintenance policy.

The OGDC [65] is based on an optimality condition presented on the same paper for coverage and connectivity, which is the assumption that $R_{c} \geq 2 R_{s}$. The main idea is to try to find clusters of nodes whose locations are as close as possible to the arrangements defined in the optimality condition. Nodes will start the process of selection by volunteer- 
ing themselves, based on a random variable proportional to their remaining energy and the current state of their neighborhoods. Once the volunteer node has announce itself, a neighbor at the proper distance will select it and then together they will look for a third node that completes the arrangement. This protocol is a distributed heuristic for covering the deployment area, based on information from neighbors 1-hop away, and does guarantee only 1 - connectivity and coverage, assuming $R_{c}=R_{s}$. It requires location information and does not provides a maintenance policy.

The ACOS protocol, presented in [85], is a localized protocol that calculates the net area covered by a sensor, which is defined as the area that is covered exclusively by a single nodes. A node is selected to remain active if the its net area is greater that a certain threshold value denoted $\varphi$, defined between 0 and 1 . This protocol uses 1-hop away information from neighbors, offers 1-coverage and assumes that $R_{c} \geq 2 R_{s}$. Some examples of area coverage protocols with K-coverage level of redundancy are the Stand Guard Algorithm (StanGA) protocol, the Coverage Configuration Protocol (CCP) and the Accurate K-Coverage Eligibility (AKCE) protocol. The CCP protocol [66] uses a property that states that an area $\mathrm{A}$ is $\mathrm{K}$-covered if the following three conditions are followed: first, the area contain intersection points between sensing areas of nodes, and between the sensing area o nodes and the boundaries of the area A; second, all the intersection points between sensing ranges of nodes are K-covered; and third, if all intersection points between sensing ranges and area boundary are K-covered. Based on the neighbor nodes' location information, each node can define if all its area has been covered by its neighbors. The Enhanced Coverage Configuration Protocol (ECCP) [86] is an enhanced version of CCP that includes two more states in their automaton in order to guarantee a better selection. 
The AKCE, presented in [87], is a protocol in which each node checks all the intersection points with its neighbors based on $R_{s}$, called R-neighbors. Then it fuses all the arcs formed, creating sectors with the same coverage degree (k's). If all sectors are covered by neighbor nodes, the node will be eligible for going into sleeping state. All the sectors that have less coverage that K, are called Decision Areas. The node calculates all intersection points between R-neighbors and between $\mathrm{R}$ - and $2 \mathrm{R}$ neighbors. If all the intersection points located in the Decision Areas have a coverage strictly higher than $\mathrm{K}$, then the node will be eligible to go to sleep mode. If at least one intersection point is not K-covered, the the node will be selected to be awake.

The StanGA protocol [88] works based on the node coverage approximation. Each node sets a random timeout during which it listens for active nodes whose distance is less than $R_{S G}$, which is a proportional value to the sensing radius. If the number of nodes inside the disk with radius $R_{S G}$ is less than $\mathrm{K}$, then the node will remain active; otherwise, if the number of neighbors is at least $\mathrm{K}$, then the node will turn itself off. This protocol is compared with PEAS [84], and three schemes presented in [89]: Nearest-neighbor, Neighbor-number and probability-based selection. The main disadvantage of StanGA is that it does not guarantee connectivity when $R_{S G} \geq R_{c} / 2$. The coverage-oriented protocols introduced in Chapter 5 of this dissertation use a similar eligibility metric to select active nodes for coverage, but guarantee connectivity in all cases.

Other examples of protocols in this category are as follows: the authors in [90, 91] calculate a maximal independent set and then find appropriate nodes to connect the sets. In [92] the nodes are selected as closely as possible to a predefined optimal topology and then hole detection and correction algorithms are executed to guarantee total coverage. In $[93,94]$ the authors use CDS techniques to provide coverage. The work presented in [69] includes a technique based on the Breadth-First Search (BFS), in which node 
$y$ is selected to be evaluated by node $x$ if $d(x, y) \leq 1.914 \alpha \pi$ with $0.5 \leq \alpha \leq 0.6$ and $R_{\text {Comm }}=R_{\text {Sense }}$.

The second main category for coverage-oriented protocols is Target Coverage. The protocols in this category are specialized in covering a set of points inside an interest area. Some examples of protocols in this category are the ones presented in [95-97].

In the EvenCover protocol [97] each node evaluates the expected density in its area based on the distance of all its neighbors in order to provide 1-coverage in the area. This metric serves as an estimate of the quality of coverage. The value of the density metric can determine three options for the node: first, if the area is very dense, then the node will go to sleep; second, if the area is somewhat dense, the node will be sent to sleep based on a random probability; and third, if the area is not dense enough, the node will remain active. The protocol assumes that the nodes are uniformly or Poisson deployed in the area. The authors in [95] propose a solution based on the construction of a Connected Dominating Set as in [52], offering both coverage-only and connected-coverage solutions, including k-coverage. In [96] the authors propose an energy-efficient solution to the Kcoverage of multiple targets in dense networks by finding disjoint topologies that cover all the targets, and then rotating them in order to preserve coverage and distribute the energy usage among different set of nodes.

\subsection{Topology Maintenance}

Given a certain number of nodes distributed in a specific area, topology construction aims at building a reduced topology that will save energy while preserving network connectivity and area coverage. Once the initial reduced topology has been created, the network starts performing the tasks it was designed for. Every activity involved in the network's 
tasks, like sensing, processing, and distributing of information has an associated cost that will consume the energy of the active nodes. Therefore, in a finite amount of time, all nodes will inexorably see their energy source depleted completely, no matter how optimal the initial reduction was. This means that in order to increase the total network lifetime, the set of active nodes, the ones in the reduced topology, cannot be active all the time. Rather, a topology maintenance mechanism should be in place to restore the current topology or build a new one - with the collaboration of formerly inactive nodes - so that all nodes can participate in the network, consume their energy in a fair manner, and increase the lifetime of the network. Accordingly,

"Topology Maintenance is defined as the process that restores, rotates, or recreates the network topology from time to time when the current reduced one is no longer optimal. It involves rotating the role of nodes as much as possible to increase the network lifetime."

\subsubsection{Classification Factors}

Topology maintenance can be exercised in different ways depending on when the topologies are built, the scope, and the type of triggering event or metric. These options are explained next.

\subsubsection{Selection Policy: Static, Dynamic and Hybrid}

Static topology maintenance techniques calculate different reduced topologies during the first topology construction process. These topologies are built and stored in memory and switched out for each other when needed. As such, static techniques have "pre-planned" 
topologies. The main goal of this approach is to distribute the activity of the network among disjoint sets of nodes. The best example to describe these techniques is making an analogy to a string of holiday blinking lights: the entire string contains a number of predefined disjoint subsets of lights that are lit one at a time, and take turns over time. As can be inferred, static techniques take additional time during the initial topology construction phase to calculate all additional topologies, but once this process is finished, the switching process is faster than if a new topology construction phase had to take place. Further, the communication overhead of each subsequent topology construction phase is also saved. Static topology maintenance techniques may have some drawbacks too. For example, it is difficult to know a priori how each of the topologies and their nodes will consume their energy. Therefore, the mechanism may choose to use some nodes in more than one topology that may not be available or will make the topology last a shorter time period than expected.

One important decision to make in the construction of the pre-planned topologies is whether or not to build completely node-disjoint topologies. This aspect must be seen along with the density of the network. For example, in a very sparse network, it might be very difficult to find more than one completely disjoint topology, and in that case, the topology maintenance mechanism would be switching the same topology over and over again. The opposite is true for highly dense networks, in which many node-disjoint reduced topologies might be found. Nonetheless, regardless of the network density, in most situations, it might be a good idea to allow shared-disjoint topologies. First, there might be critical nodes that, if not included in the topologies, would make static techniques look like topology construction algorithms. Second, shared-disjoint topologies allow new nodes to be included in the network with the possibility of extending its lifetime. In either case, a series of pre-planned topologies are stored in the network and switched based on 


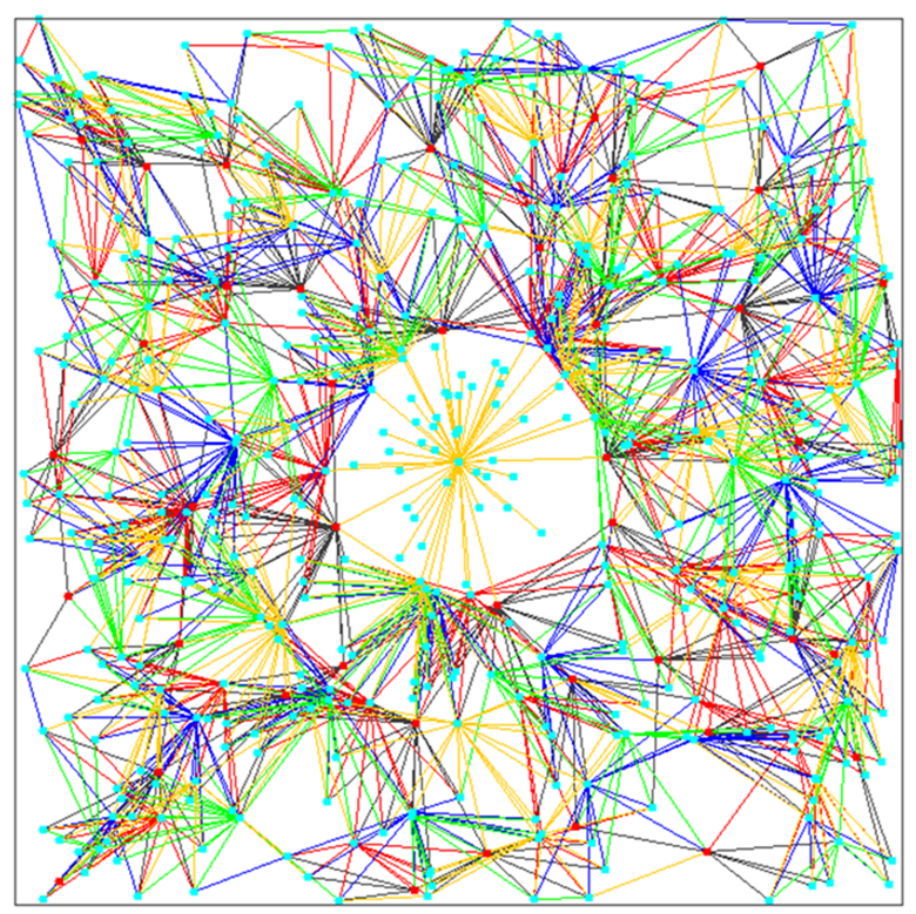

Figure 2.9: Multiple node-disjoint CDS trees over the same network.

the established triggering criteria. Figure 2.9 shows an example of the number of nodedisjoint reduced topologies that can be created to implement the Static Topology Rotation approach.

Contrary to static topology maintenance techniques, Dynamic topology maintenance techniques do not make a priori calculations to determine the topology that will become active when the current one is no longer appropriate. Instead, dynamic techniques make those calculations "on-the-fly". As such, dynamic techniques are usually more time and energy consuming since they may have to run the topology construction process several times. Therefore, the energy efficiency of the underlying topology construction algorithm plays an important role. However, since dynamic techniques consider the current status of the network when making the new calculations, the topology construction process usually 
chooses an optimal or close to optimal topology every time it is run, resulting in better or more adequate subsequent topologies, as compared with static techniques.

Finally, Hybrid topology maintenance techniques are a combination of static and dynamic techniques. The algorithms in this category work in two different stages. In the first stage, the protocol calculates all different reduced topologies during the first topology construction phase (static approach). The second stage is when the network determines that the current reduced topology cannot be established because the sink has no connectivity with any active nodes (dead topology), then mechanism executes a topology construction protocol in order to find a new topology "on the fly" (dynamic approach). The protocols in this category show some advantages compared to the previous techniques, like it can use resources that the static-only protocols will not be able to use, and that the network may last longer than when using a dynamic approach because the rotation process consumes less overhead than a complete new construction process. However, the hybrid techniques also show a disadvantage that none of the static- or dynamic-only have: depending on the selection of the triggering criteria, a crippled reduce topology can last a long time, affecting the level of service in connectivity and coverage, before it gets to the point where it needs to be restored globally. These issues will be addressed in detail in Chapter 6 .

\subsubsection{Level of Involvement: Global and Local}

The second aspect is related to the scope of the technique: which nodes, or which part of the network, are involved in the execution of the topology maintenance algorithm. The scope can be global or local. While global techniques consider all the nodes in the network in order to make a global-optimal decision, local techniques only consider a small subset of the nodes in order to make a decision in a local-optimal fashion. Therefore, 
global techniques switch the entire topology, and local techniques only switch a portion of the network, such as a branch of a tree, a cluster, or even just a single node.

Static techniques are usually global in nature, as the calculation of replacements for more localized portions of the network may take an excessive amount of resources. One alternative to make static local techniques more attractive is to increase the scope of the portion of the network to switch. For instance, in a tree-based network, several different tree branches might be found during the first topology construction phase and, upon the triggering criteria, an entire branch is switched. However, as the scope is increased, local techniques become more and more similar to global techniques.

Global dynamic techniques run a network-wide new topology construction process every time the topology needs to be changed and, therefore, switch the entire topology. Local dynamic techniques, on the other hand, run a more localized process meant to calculate and establish a new branch of a tree, a cluster, or a specific link or small set of links. Contrary to global techniques, local dynamic techniques might be able to increase the network lifetime more than either static or dynamic global techniques, as it may be possible to restore a local failure with just a few computations and a small number of messages.

\subsubsection{Triggering Mechanisms}

Whether the topology maintenance mechanism is static, dynamic, or hybrid, global or local, there is one important question related to all: what is the criterion or criteria that will be used to trigger the process of changing the current topology? The triggering criteria, which may have important implications in terms of energy savings as well as coverage, reliability, and other important metrics, may be based on one of the following choices: 
- Time-based: In time-based topology maintenance, the current topology is changed when a timer expires. The amount of time is usually fixed and pre-defined, and is a very critical variable. Too short a time between changes may cause unwanted, extra overhead as a consequence of switching the topology more often than necessary. On the other hand, a very long time between changes may use a suboptimal network longer than necessary, with the possibility of losing important events due to its poor coverage.

- Energy-based: Given the energy limitations of wireless sensor devices, it makes sense to switch the topology when the energy level of the nodes goes below a certain threshold. Again, the choice of the energy threshold is very critical for the same reasons explained before. Changing the topology too often may end up spending more energy than the energy that is supposed to be saved by topology maintenance, thereby defeating its purpose. A very low threshold, on the other hand, will make certain critical nodes unavailable for upcoming topologies.

- Random: In random-based topology maintenance, the current topology is switched using a random variable.

- Failure-based: A failure-based technique triggers the process of changing the current topology after a node, or a number of nodes, have failed. These techniques require the existence of failure detection and notification mechanisms.

- Density-based: Another criterion might be based on the density of the network. A similar metric might be the node degree of the network or the node degree of some important nodes. 


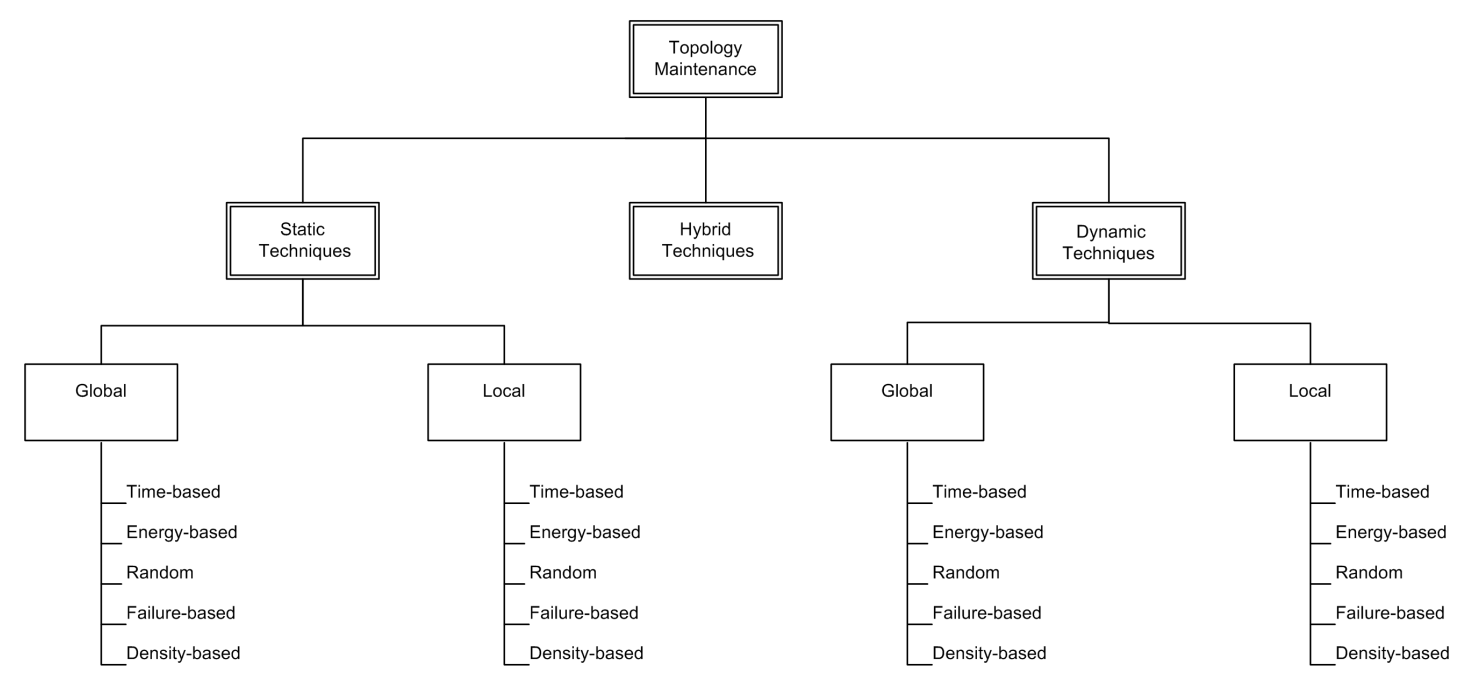

Figure 2.10: Classification of topology maintenance.

- Combinations: Combinations of these criteria could be used as well. For example, the topology maintenance could be activated based on energy and failure, or time and energy.

Figure 2.10 shows a complete taxonomy of topology maintenance that also includes some of the possible triggering criteria.

\subsubsection{Examples of Topology Maintenance Algorithms}

The topology maintenance algorithms that are introduced in this dissertation will be described in Chapter 6. In this section, some of the most relevant topology maintenance protocols will be presented. In literature, there are not many topology maintenance techniques that could be classified as static global. One approach, presented in $[69,96]$, is based on the static maintenance concept. In general, the static maintenance scheme requires special attention in the use of the random- and pattern-based approaches for synchronized radios to wake up the nodes and build the reduced topologies. 
Once the initial reduced connected-coverage topology is built using any of the mechanisms mentioned above, the chosen mechanism is run iteratively using only the remaining nodes, or those nodes that have not been part of any reduced topology calculated before. Since the work focuses on building the maximum number of node-disjoint topologies, it does not include any performance evaluation of the presented technique in terms of number of active nodes, nor does it mention anything about the triggering criteria that could be utilized to switch the topologies.

In the case of dynamic global topology maintenance techniques, there are many topology construction mechanisms that embed and utilize this technique without formally calling it topology maintenance. One example is that of the LEACH protocol described in Section 2.2.2. LEACH changes the entire network topology periodically, in rounds; therefore, it is a dynamic global technique with a time-based triggering criterion. As described in Section 2.2.2.2, it also includes a clusterhead selection metric that guarantees that the nodes consume their energy in an even and fair manner.

There are several techniques available in the literature that perform dynamic local maintenance of the network topology without being explicitly categorized as dynamic local topology maintenance techniques. For example, in [98], the authors propose a fault tolerant topology maintenance methodology in which they select a set of backup nodes that will maintain backbone connectivity replacing nodes in case of failure. The selected backup nodes will turn on when a failure is detected in one of the active neighbors of a non adjacent active node, creating an alternate route to reach the active node in case it was disconnected from the network. Another dynamic local failure-based technique is presented in [99]. The work proposes to increase the transmission range of a neighbor of the failed node. The neighbor is selected either randomly or based on the remaining energy of the nodes. 
In [100], the authors present an evaluation scheme for node failure in static wireless networks. The evaluation consists of periodical requests from each node to all the neighbors in range. The recipient of the request must execute a test task and return the result to the requester. If the result is correct and the message is received during an expected interval, then the node is considered available. The authors in [101] extended this evaluation procedure to mobile units, including recovery after failure.

The Geographical Adaptive Fidelity (GAF) algorithm introduced in [102] is another example of a dynamic local topology maintenance technique. GAF is based on the known observation that in dense networks multiple paths can be established between nodes, therefore, there is the possibility of turning some nodes off without jeopardizing the network connectivity. GAF uses node location information and a virtual grid to introduce the concept of node equivalence in which all nodes within the same virtual grid square are considered equal in terms of their ability to route packets. Nodes in the same grid then coordinate among themselves to determine who will perform the routing function on their behalf and for how long. This negotiation is based on an application-dependent ranking procedure that chooses as the active node the one with the most energy. The active node then broadcasts the amount of time it will be active, which is calculated based on the amount of remaining energy. Once the active node consumes half its energy, the nodes wake up and the negotiation procedure starts again. At that time, it is very unlikely that a node who has been active recently will be selected again since it was active while the other ones were sleeping, achieving the desired energy consumption balance. The SPAN algorithm introduced in [49] is similar to GAF in the sense that each node decides to be part of the routing backbone based on the number of neighbors and its amount of energy. In SPAN, backbone nodes are called coordinators, and also rotate with time. One difference between the two algorithms is that SPAN does not need location infor- 
mation. SPAN is based on HELLO packets by which nodes know who their neighbors and coordinators are. If two neighbors of a non-coordinator node cannot reach each other either directly or through one or two coordinators, then the node should become a coordinator. In order to resolve contention problems when more than one node volunteers as a coordinator, SPAN delays the coordinator announcements by a time that considers the number of nodes among these neighbors that would be connected if the node were to become a coordinator, and the remaining energy in the node. Then, periodically, each coordinator checks if it should continue being a coordinator. In order to ensure fairness, the coordinator withdraws itself as a coordinator if every pair of neighbor nodes can reach each other via some other neighbors.

The Adaptive Fidelity Energy-Conserving Algorithm (AFECA) proposed in [103] is also very similar to GAF and SPAN. AFECA shares the same motivation and approach to topology maintenance of GAF and SPAN but uses a different switching metric: neighbor density. Each node estimates its neighbor density by maintaining a list of the nodes it is able to hear, then, it calculates its sleeping time using a proportional factor of the number of neighbors. 


\section{Chapter 3: Methodology}

This chapter describes the methodology used for the performance evaluation of the different topology construction and maintenance protocols introduced in this dissertation. Both analytical and simulation-based evaluations are included. This chapter is divided in two sections. The first section presents a new analytical solution to the minimal connected dominating set problem using a mixed integer programming approach (MIP), which serves as a centralized optimal alternative to the heuristics proposed later in Chapter 4. The second section describes metrics, factors and levels utilized in the simulationbased performance evaluation of the protocols, plus the definition of the model for energy consumption. The performance evaluations are then included in the appropriate chapters

\subsection{An Analytical Solution to the MCDS Problem}

Previously, in Section 2.2.2.1, the MCDS problem was formally defined as a connected set of nodes $D \subset V$ in a graph, such that every node in $V$ either belongs to $D$ or has at least one link to a node in $D$. The MCDS problem is a particular case of the Minimal Dominating Set (MDS) problem, that has been solved using both analytical and approximation approaches. However, in the case of the MCDS problem, not many analytical solutions have been proposed to solve this problem, compared to the great majority of approximate solutions available in the literature. 


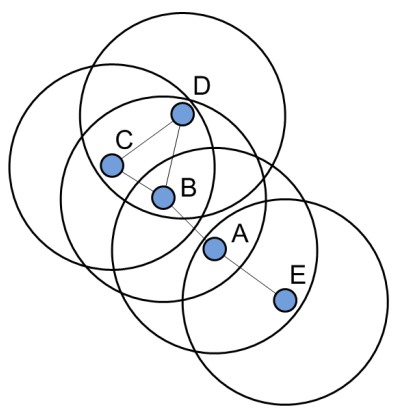

A. Initial Graph

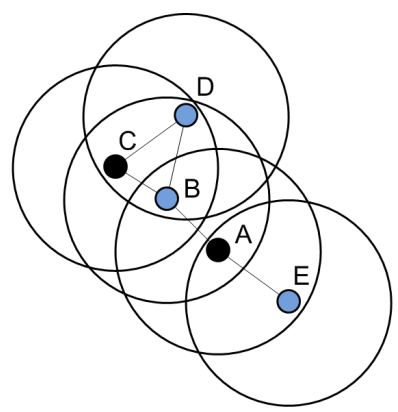

B. MDS 1

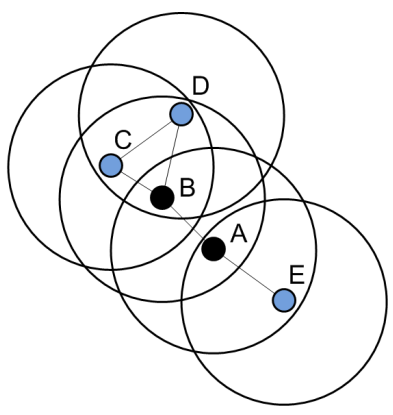

C. MDS 2

Figure 3.1: Examples of MDS solutions.

Two approaches have been found in the literature. The first one, used in [37], divides the MCDS problem into two sub-problems: finding the MDS of the graph, and then finding a spanning tree over the graph that connects the MDS nodes. The "divide and conquer" approach may reduce the total complexity of the problem, which is an important advantage in large scenarios; however, an improperly divided solution could lead to scenarios like the one presented in Figure 3.1.

The initial graph is a very simple one. Two MDSs can be obtained from the given graph and, given that the MDS linear programming formulation has no weights on the objective function, as in [37, 104], any of the two solutions are feasible optimal solutions. However, if solution MDS 1 is fed to the second stage, it will need to include more active nodes in order to provide connectivity among the dominating nodes, which as it can be seen in the solution MDS 2, is not necessary. As said before, an adequate decomposition of the MCDS problem could provide a successful formulation that will overcome this scenario and provide the optimal solution.

The second approach for an analytical solution to the MCDS problem is presented in [105]. This formulation is based on two conditions that must be accomplished by every CDS: On every subset of nodes $S \in V$, there must be at least one gateway node that has a link to a node in the set $V / S$ (vertex-cut condition), and for every non-gateway node $u$, 
a message generated from any neighbor of $u$, say node $v$, must have a way to reach every other neighbor of $u$ (neighbor connectivity condition).

The formulation proposed by the authors is based on the solution of the minimum vertex cut problem for all possible subsets $S$ of $V$ with the vertex-cut condition, which they prove will produce a MCDS set. Given that the number of all possible subsets of $V$ grows exponentially with $|V|$, the authors propose an iterative and incremental constraint generation scheme that increases the number of subsets considered on every iteration in a linear fashion. The iterative process starts with the optimal solution based on the current subsets. If the optimal solution of the vertex cut problem is a CDS, then it must be a MCDS, and the program stops. If the solution is not connected, then the authors include new constraints based on the non-optimal solution and will rerun the optimal solution.

Both the previous formulations do not show enough clarity in their implementation to allow an effective replication of their model, and they also show dependence on heuristic processes in order to provide an approximation to the optimal solution of the MCDS problem, and not the optimal one itself. This lack of guarantee in finding the optimal solution is the main motivation for the introduction of the new formulation presented in this dissertation.

MIP-MCDS is a mixed integer programming formulation that finds the optimal solution to the MCDS problem. There are two main sets of constraints that guarantee dominance over the network and connectivity among the dominator nodes. The formulation is based on the basic definition of the MCDS problem, which makes it very clear to understand and replicate, as can be seen in Section 3.2 where it is introduced in detail. The approach used in the MIP-MCDS solution is based on the assumption that a MCDS can be defined as the minimum cardinality subset of non-leaf nodes among all possible 
trees that can be obtained from a connected graph. In order to prove this statement, some other statements must be also proved.

Theorem 1. Every possible tree that can be constructed from a connected graph with $n>2$, will contain at least 4 solutions to the CDS problem. It is known that every connected graph contains at least one tree that includes all nodes. This is simply proven by the fact that acyclic structures can be obtained from simple algorithms like the Breath First Search (BFS), Depth First Search (DFS) or the Minimal Spanning Tree (MST). Furthermore, by definition, the complete set of nodes of a graph is also a CDS. The network topology that offers the worst case for a connected dominating set can be seen in the linear graph, in which all the intermediate nodes between the extremes must be selected as dominators in order to guarantee connectivity. As stated before, this is a solution of the CDS problem for this graph. The other three solutions come from the scenarios in which the nodes in the extremes are dominated, and in two scenarios in which just one of them was selected to be a dominator and the other is dominated. These four scenarios are the only possible solutions for a CDS in a path topology. Any other topology is expected to have more leaf nodes, which can produce more combinations for solutions of the CDS.

Corollary 1. Every possible tree that can be constructed from a connected graph with $n>2$ will contain $2^{L}$ solutions to the CDS problem where $L$ is the number of leaf nodes in the tree. In general, the total number of connected dominating sets contained in a tree can be calculated by all the possible combinations of dominating-dominator states on the leaf nodes. This value is equivalent to $2^{L}$, where $L$ is the number of leaf nodes in the tree, i.e., all nodes with cardinality 1 in the tree. 
Corollary 2. The minimal cardinality CDS in a tree is $n-L$. All non-leaf nodes are necessary in the CDS because they have at least one node under their dominance. The inclusion of any leaf node in the solution of a CDS will result in a redundant solution. This means that the exclusion of all leaf nodes will produce the minimal cardinality CDS of a tree. In the case when a graph is a path, it is trivial to observe that only one tree can be constructed from the original topology, in which the number of leaves corresponds to the two nodes in the extremes that do not offer dominance over any node; therefore, having that $L=2$, then the minimal cardinality CDS solution of that tree will be the minimal CDS of the graph which is $n-2$. Now, the other extreme case is when the initial graph $G$ is a fully connected graph in which each node $u$ has an edge toward every other node in the graph. It is easily seen that if the tree is selected to be a star topology, a single node is able to dominate every other node in the graph, so the MCDS solution to this particular tree has a cardinality of 1 . This statement implies also that the optimal solution of the MCDS problem is a subtree from one or more of the possible trees that can be generated from a connected graph. The conclusion on this Corollary is supported also by Theorem 6.16 in [104].

Theorem 2. Not all possible trees that can be constructed from a connected graph contain a solution to the MCDS of the original graph. Based on the result of Corollary 2, it can be seen that every possible tree obtained from a graph contains a CDS with minimal cardinality of $n-L$ dominant nodes for their particular tree; however, the minimal cardinality CDS solution for a particular tree is not necessarily the minimal cardinality CDS of the initial graph. Assume graph $G$ as a fully connected graph. As shown in Corollary 2, when having a star topology tree, the minimum cardinality CDS and the optimal CDS of the graph has a size of 1 . Nevertheless, one of the possible trees that can be constructed from a fully connected graph is a path graph 
which, as it was shown also in Corollary 2, has a MCDS with a minimum cardinality of $n-2$ which is greater than the minimum cardinality of the MCDS from the tree with star topology.

Based on Corollary 2, it can be seen that the construction of a minimum tree that provides dominance of the graph is a legitimate approach to finding the optimal solution of the minimal connected dominating set problem. However, Theorem 2 shows how the process of finding the tree that contains the optimal CDS is not trivial.

The main advantage of including both connectivity and dominance as a single problem is that all nodes selected to be active are potential dominators, as opposed to the dominatorsgateways approach in which the dominance capability of the gateway nodes is not used. This characteristic eliminated the possibility of having cases like the one in Figure 3.1b in which node B could have been selected not only as a gateway but also as a dominator. The formulation presented in the next section is based on the search of the dominating subtree. The structure of a tree was selected because: first, the number of links is known to be always $n-1$, being $n$ the number of nodes in the tree; and second, because no matter what shape the final graph of the dominators takes, if it is a connected graph, then a tree can be calculated from the total set of edges.

\subsection{The MIP Approach for the Minimum Connected Dominating Set Problem}

The solution presented in this section is based on a mixed integer programming (MIP) formulation of the Minimum Connected Dominating Set problem starting from the sink node. This variation is based on the fact that, in real applications, the sink node must be always active and that it should be the center of the logical infrastructure in order to 
reduce the distance from all the nodes to itself. A general solution to the CDS problem can be solved using a similar approach, but it will not be included in this dissertation. As stated before, this formulation is based on two constraints: domination and connectivity. The domination constraint is based on the Minimum Total Dominating Set (MTDS) problem, as presented in [104]. The MTDS problem differs from the regular MDS problem in the sense that every node, including the dominator nodes, must have at least one dominator node in their neighborhoods. This constraint guarantees that there will be no isolated dominator nodes, which is a necessary condition of any MCDS solution but insufficient, because it does not guarantee connectivity in the subset of dominant nodes; for example, the final reduced topology may be a forest graph made of islands of connected components built from dominator nodes, in which the dominator nodes are not isolated, but disconnected.

The connectivity constraint is based on a technique called the "flow of tokens". The "tokens" represent the right for a node to become a dominator node, and they flow though the network creating a tree of dominator nodes. The minimum tree that dominates all nodes in the network will be the MCDS that starts in the sink. The "flow of tokes" technique is defined based on the following rules:

- The sink node is assumed to be the unique source of the tokens and it will posses $n^{\prime}$ tokens, where $n^{\prime}$ is assumed to be the minimum number of required dominator nodes to cover the complete network, including the sink node.

- The distribution process starts with the sink consuming one token and distributing $n^{\prime}-1$ tokens among its neighbors selected to be dominators. 


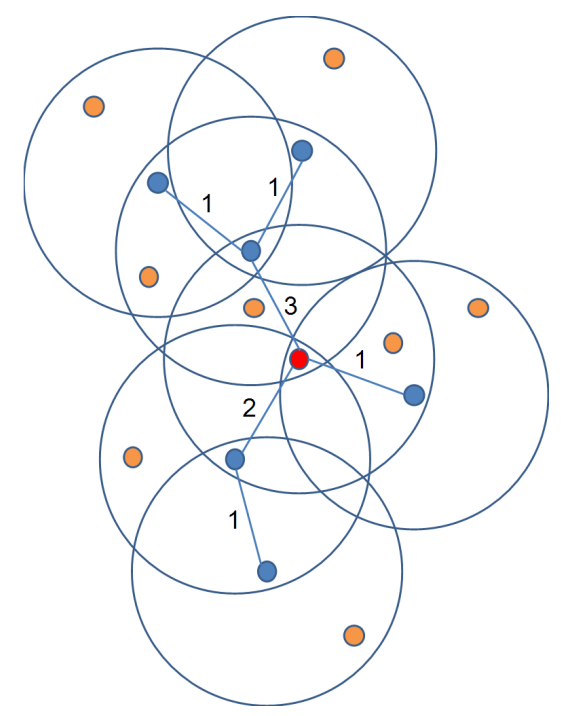

Figure 3.2: Example of the "flow of tokens" technique.

- Each dominator node $w$ will receive a number of tokens $n^{\prime}(w) \leq\left(n^{\prime}-1\right)$ from another dominator node, will consume 1 and then it will distribute $n^{\prime}(w)-1$ tokens among its neighbor nodes selected to be dominators also.

- Dominator nodes are the only ones who can distribute tokens.

- A dominator node that receives just 1 token cannot select any more nodes to be dominators.

- A node cannot be a dominator if it does not receive a token from another dominator node.

- There can be flow of tokens only where direct links exist between nodes

An example of the execution of the "flow of tokens" technique can be seen in Figure 3.2. The sink node (in the center) generates the flow that will reach all active nodes. In the example it can be seen how the sink node distributes 6 tokens among its neighbors, which will do similarly with the extra tokens they received. 
The mathematical formulation assumes that there exists a connected graph $G=(V, E)$, where $n=|V|$ is total number of nodes in the network and $a d j$ is the a adjacency matrix of size $n \times n$ which contains 1 if there is direct communication between the nodes $i$ and $j$, or 0 otherwise, for every pair $i, j \in V$.

The problem has two sets of variables: the binary array $y$ with size $n$, in which $y(i)$ represents if the node is selected to be dominator with a 1 , and 0 otherwise, and the integer array flow with size $n \times n$ in which flow $(i, j)$ contains the amount of tokens that flow from node $i$ to node $j$.

Equation 3.1 shows the formulation of the mixed integer programming version of the minimum connected dominating set based on the "flow of tokens" approach, which is one of the contributions of this dissertation.

$$
\begin{array}{lcc}
\min & n^{\prime}=\sum_{i} y(i) & \\
\text { s.t. } & \sum_{j} f(s, j)-\sum_{i} f(i, s)= & n^{\prime}-1 \\
& \sum_{j} f(i, j) \cdot \operatorname{adj}(i, j)-\sum_{k} f(k, i) \cdot \operatorname{ad} j(k, i)= & -1 \cdot y(i) \\
\forall i, & \sum_{j} f(i, j) \cdot a d j(i, j) \leq & n \cdot y(i) \\
\forall i, & \sum_{j} a d j(i, j) \cdot y(j) \geq & 1
\end{array}
$$

The objective function of this problem is the minimization of the number of active nodes, as shown in Equation 3.1. The constraints of the minimization function are the following: 
- Constraint 3.2 determines that the number of tokens that flow from the sink is $n^{\prime}-$ 1 , which is the number of dominators minus 1 .

- Constraint 3.3 determines that the difference between incoming and outgoing flow is -1 or 0 , depending if the node is a dominator or not, respectively. This restriction also controls that dominated nodes have no flow and that flow can only occur between nodes that are adjacent.

- Constraint 3.4 determines that the outgoing flows is less or equal than the total number of nodes in the topology for active nodes, and 0 for inactive nodes. This restriction also limits the existence of flow of tokens only if the nodes are adjacent.

- Constraint 3.5 determines that each node must have at least one dominator in its neighborhood. This constraint guarantees that set of selected nodes is a total dominating set.

The current formulation of the MIP-MCDS problem is an initial version to the analytical solution of the MCDS problem, whose purpose is only to serve as a tool for comparison and evaluation of the approximation algorithms presented in this dissertation. The formulation still has some room for improvement in order to reduce the execution time. A pre-processing scheme could be implemented in order to eliminate some trivial decisions; for example, turning off immediately all nodes with node degree equal to 1 , because they are destined to be dominated. A more complete analysis and further improvements are part of the future work on this topic. In addition, due to the NP-Hard nature of the MCDS problem, this formulation is not scalable for large networks, which is why most of the discussion in this dissertation revolves around heuristic solutions.

The MIP-MCDS optimization model was tested using the topology control simulator Atarraya, included in this dissertation, and the optimization program called Gurobi $($ ) de- 
veloped by Gurobi Optimization ${ }^{1}$. The results of the analytical comparison of the optimal solution against the proposed heuristics will be shown in Section 4.4.

\subsection{Performance Evaluation: Assumptions, Metrics, Factors and Levels}

This section presents the general assumptions, performance metrics, factors and levels utilized in the performance evaluations presented in this dissertation. The evaluations as well as the characteristics of the particular scenarios used for each set of experiments will be presented in the performance evaluation section of each chapter.

\subsubsection{Assumptions}

The following are the assumptions made in this dissertation:

- All nodes are located in a two dimensional space and have a perfect communication coverage disk.

- All nodes have equal communication ranges and all links are bidirectional.

- All nodes have a single sensor and have a perfect sensing coverage disk.

- Nodes have no information about their position, orientation, or neighbors.

- The initial graph, the one formed right after the deployment using the maximum communication range, is connected.

- Distances between nodes can be calculated as a metric perfectly proportional to the Received Signal Strength Indicator (RSSI).

\footnotetext{
${ }^{1}$ http://www.gurobi.com/
} 
- There is no packet loss at the Data Link Layer.

- There is a mechanism by which a node can be awakened when its radio is off.

- The network duty cycle is $100 \%$ - The network is always active.

- The time units of the simulation clock are approximately 1 second.

\subsubsection{Performance Metrics}

In order to provide a tangible comparison among topology control alternatives, the following performance metrics were chosen. Some of them are exclusive to the construction or the maintenance processes.

- Number of active nodes: this metric measures the quality of the selection policy for nodes. In addition, the amount of active nodes selected by the algorithm has a direct impact on the lifetime of the network.

- Number of messages: this metric shows the overhead of the protocol in terms of message complexity, which is also related with the scalability of the protocol and the energy consumption.

- Ratio of energy spent: this metric shows the cost of the protocol in terms of energy; in other words, how much energy is spent in the execution of the protocol.

- Ratio of covered area: this ratio is important for comparing coverage-oriented protocols in order to compare effectiveness of their selection policies.

- Network lifetime: this metric is useful especially in comparing topology maintenance protocols, and shows the behavior of some of the previously mentioned 
metrics in the time domain, in order to obtain an average behavior of the use of the resources in the network in the long run.

\subsubsection{Factors and Levels}

The evaluation of topology control protocols was performed in different scenarios, in order to obtain a general idea of the behavior of the protocols under certain conditions. The list of factors that were used to define the different scenarios is the following:

- Number of nodes: this parameter determines the size of the topology. The variation of this parameter helps to determine the scalability of the protocols. The network sizes used on the experiments varied based on the evaluated metric, from very small topologies with only 5 nodes, to very dense topologies with 4000 nodes.

- Side of the area $L$ : this parameter defines the size of the deployment area. The area is assumed to be a square of side L. This factor varied between 50 and 600 meters, depending on the definition of each particular experiment.

- Communication range $R_{c}$ : this parameter is very useful because it has an implication in other parameters like average node degree. The levels of this factor were calculated mostly using the Critical Transmission Range (CTR) formula [6]. The CTR is the minimal radius that w.h.p. produces a connected topology given the size of the network and the area side $L$. Based on the CTR, the communication range takes values of 1 to 3 CTRs. In some cases, the levels were calculated as a ratio of the sensing range. In this fashion, this factor takes values of $1, \sqrt{3}$ and 2 . 
- Sensing range $R_{s}$ : this parameter is important determining the area of coverage of a single node. The levels of this factor were statically defined or were defined as a certain ratio of the side of the area $L$.

- Number of Virtual Network Interfaces (VNIs): this parameter is useful for topology construction and maintenance protocols that contemplate the use of multiple reduced topologies. All the experiments that used multiple VNIs, assumed a value of 3 VNIs in the network.

- Level of redundancy K-Comm and K-Cov: these parameters determine the level of redundancy in communication and coverage. All the experiments assume a value of $\mathrm{K}-\mathrm{Comm}=1$ and $\mathrm{K}-\mathrm{Cov}=1$.

- Node location distribution: the distribution of the nodes in the area plays a very important role in the performance of the protocols. Even though many assume uniformly random distribution, some require specific densities in every section of the deployment area.

- Network load: the amount of messages that every active node will be sending during the operation of the network. This could be constant and periodic, or could be variable depending on the occurrence of an event. All experiments assumed a network load of 1 message every 10 seconds per active node. In addition, there was no data aggregation technique in order to reduce the load on the network.

- Packet size: all experiments use two different message sizes: short messages, assumed to be control packets of 25 Bytes long, and long messages, assumed to be data or special long control packets of 100 Bytes long. 
- Initial energy: this parameter represents the initial energy reserve that a node has at the beginning of the simulation. The value assumed for this parameter is 1 Joule per node, as in [55]. This value is considerably small compared with the real amount of energy in an AA battery, however it was selected for convenience in order to reduce the simulation time. It is expected that the performance evaluation of the network with real energy reserve values will be proportionally similar to the results presented in this dissertation.

\subsubsection{Energy Model}

In order to assess the lifetime of a given topology, it is important to include a model to drain the nodes' energy every time they perform any action. The energy model used in this dissertation to model the energy consumption of the nodes is based on Equations 3.6 and 3.7, as presented in [55], which is mostly based on the actions of transmmiting or receiving data.

$$
\begin{aligned}
& E_{T x}=E_{\text {elec }}+E_{\text {amp }} * R_{\text {comm }}^{2} * \pi \\
& E_{R x}=E_{\text {elec }}
\end{aligned}
$$

where $E_{T x}$ is the energy spent to transmit 1 bit, $E_{R x}$ is the energy to receive 1 bit, $E_{\text {elec }}$ is the energy used by the electronic components of the radio, and $E_{a m p}$ is the energy used by the radio amplifier. The second term is proportional to the square of the transmission range that wants to be achieved by the radio signal. Despite the simplicity of this energy model, it is still commonly used in the literature of wireless sensor networks. In the performance evaluations presented later in this dissertation, it is assumed that idle listening 
Table 3.1: Parameters for the energy model.

\begin{tabular}{|c|c|}
\hline Initial energy source & 1 Joule \\
\hline$E_{\text {elec }}$ & $50 \mathrm{~nJ} / \mathrm{bit}$ \\
\hline$E_{\text {amp }}$ & $10 \mathrm{pJ} / \mathrm{bit} / \mathrm{m}^{2}$ \\
\hline
\end{tabular}

energy consumption is negligible. The values of the parameters for the energy model are summarized in Table 3.1. 


\section{Chapter 4: The A3 and A3Lite Topology Construction Protocols for Connectivity}

In Chapter 2, a large number of solutions for topology construction were discussed, both for connectivity only and for connected coverage. The main contributions presented in this dissertation are the definition and implementation of a new family of simple topology construction protocols for wireless sensor networks. The $A 3$ family of protocols is composed of four protocols: A3 and A3Lite, focused on achieving connectivity while reducing the number of active nodes as much as possible, and A3Cov and A3CovLite, which try to increase the amount of area covered by the reduced topology in the cases where $R_{\text {Comm }} \neq R_{\text {Sense }}$, without increasing dramatically the number of active nodes. This chapter will be dedicated entirely to the first two connectivity-oriented protocols. Even though it was discussed that coverage must be a critical consideration on any topology construction protocol, the assurance of connectivity is also fundamental because a network that offers perfect coverage but lacks the means to transmit the information is useless. That is the reason why the $A 3$ and $A 3$ Lite connectivity-oriented protocols are the foundation of the $A 3 C o v$ and $A 3 C o v L i t e$ coverage-oriented protocols presented in Chapter 5. 


\subsection{Introduction}

The algorithms presented in this chapter belong to the category of hierarchical schemes that create a Connected Dominating Set (CDS) to reduce the topology, as defined in 2.2.2.1. Among the wide range of topology construction algorithms shown on the second chapter, this category was selected based on the advantages it offers:

- The nodes selected to be part of the CDS are sufficient to provide connectivity to the network. This gives the possibility of turning off every non-selected node, while providing connectivity and a reduced degree of sensing coverage of the deployment area, depending on the ratio between the sensing range and the area side.

- If the non-selected nodes are sent to sleep, their energy will be used for future maintenance of the network, when the initially selected nodes start to finish up their energy reserve. These savings are not possible on techniques that are based on just altering the transmission power.

- When most of the nodes are turned off, the node degree is reduced, so interference and collisions are reduced considerably. This surely has an impact on the packet delay and throughput of the network.

The focus of this chapter is to present two simple topology construction algorithms that guarantee connectivity, with low message and computational complexity: A3 and A3Lite. The A3 algorithm, whose name comes from "A Tree", is the first member of the family of topology construction protocols presented in this dissertation. As it name implies, this algorithm creates a tree that covers every single node in the topology and provides a communication backbone for the network, and all the non-selected nodes are sent to a low 
energy consumption state so their energy will be preserved for future use. Even though the idea is definitely not new, the methodology and selection metric certainly are.

The second algorithm is the A3Lite protocol, which is an evolution of the A3 protocol. The idea with the second protocol is that, while using the same basic principle to create the tree, A3Lite uses less messages, reduces the computational complexity and even produces a reduced topology with less active nodes than its predecessor.

These protocols will be tested against two well-known topology construction protocols also presented on Chapter 2: EECDS [44] and CDS-Rule-K [52-54]. Furthermore, the performance of A3 and A3Lite will be also compared to their extended version for coverage (A3Cov and $\mathrm{A} 3 \mathrm{CovLite}$ ) and with the optimal deployments for connected coverage

presented in [69] in Chapter 5, based on the fact that when $R_{\text {Comm }}=R_{\text {Sense }}$, the problem of connectivity is similar to the problem of connected coverage, which discussed in the next chapter.

\subsection{The A3 Algorithm}

Using a more formal definition, the A3 protocol [106] produces a tree that is an approximate solution to the Minimal Connected Dominating Set problem (MCDS), which is proved to be NP-Hard in [36]. One of the advantages of the heuristic nature of this solution is that it can be executed in a distributed way in the topology, opposed to a centralized processing that the optimal mixed integer programing solution presented in Section 3.2. As expected the cost of the approximate solution is represented by the extra active nodes selected that the optimal solutions would not have chosen. This topic will be discussed in detail later in Section 4.4, in which the solutions provided by the MIPand heuristic-based approaches will be compared. 

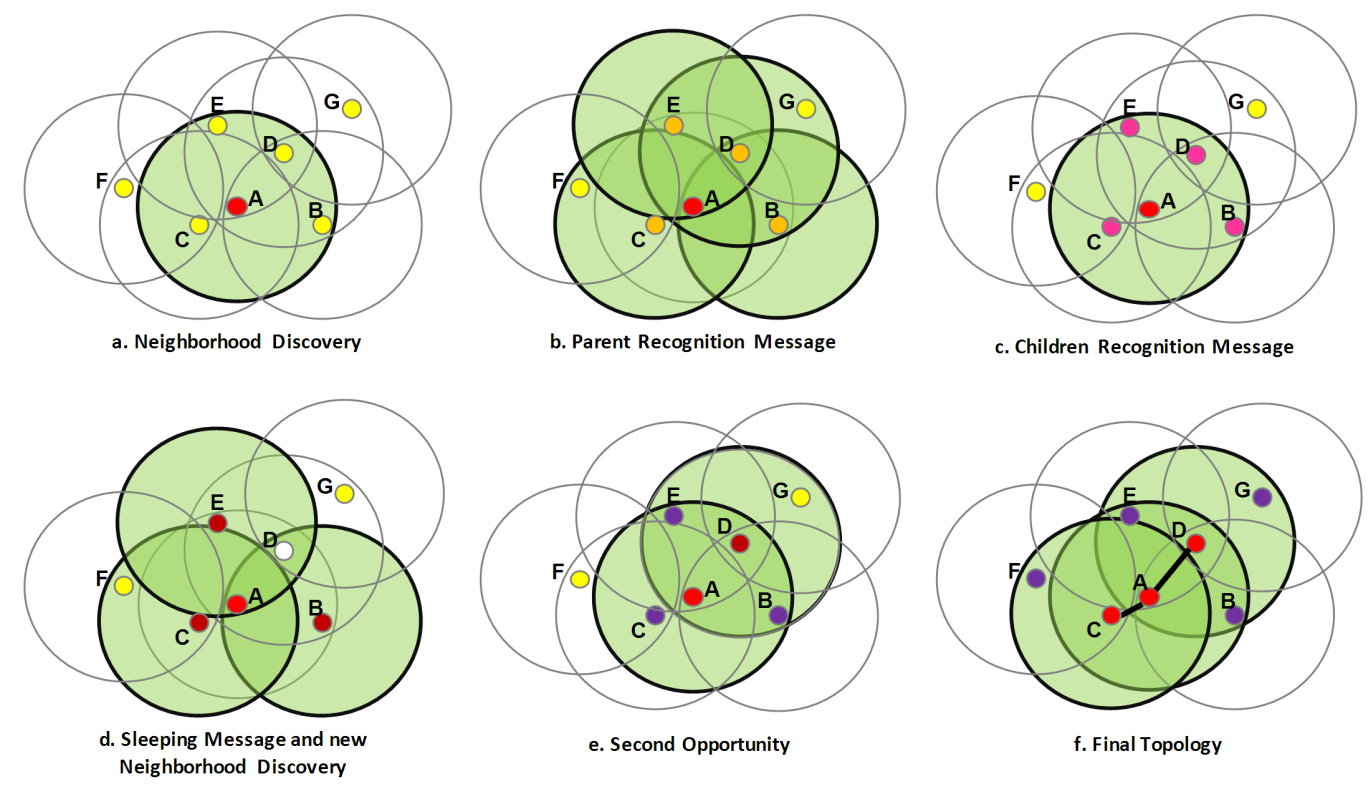

Figure 4.1: The A3 algorithm.

The A3 algorithm assumes that the nodes have no knowledge about the position or orientation of their neighbors; therefore, the nodes lack an exact geometric view of the topology. On the other hand, nodes are able to determine the distance to their neighbors based on the signal strength. The distance plays a major role in the selection of the nodes based on the belief that, the more spread out the nodes are, the more area and unvisited nodes they will cover. The A3 algorithm consists of the Neighborhood Discovery, Children Selection, and Second Opportunity processes.

\subsubsection{The Neighborhood Discovery Process}

Once the nodes are deployed, the tree building process is started by a preselected node, usually the sink node. The sink's initial state is Active Candidate, while all the other nodes are in the Initial state. 


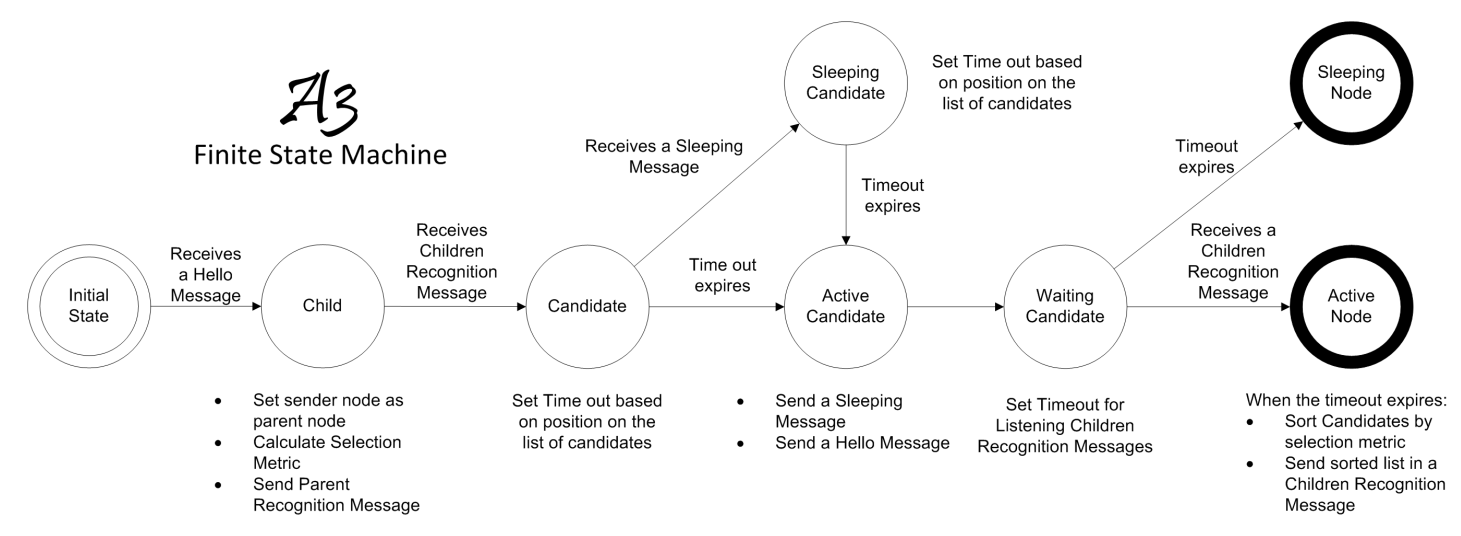

Figure 4.2: The A3 protocol finite state machine.

The sink, node A in Figure 4.1(a), starts the protocol by sending a Hello Message. This message allows the neighbors of A to recognize their dominant or "parent" node. In Figure 4.1(a), nodes B, C, D, and E receive the message. Nodes F and G are out of reach from node A.

If a node has received a Hello Message previously, it ignores any other messages of this kind; otherwise, the receiver sets its state as Child, adopts the sender as its "parent node", calculates the selection metric (explained later in Section 4.2.3.1) and answers back with a Parent Recognition Message, as shown in Figure 4.1(b), which includes the calculated metric. The selection metric is calculated based on the signal strength of the received Hello Message and the remaining energy in the node, and will be used later by the parent node to sort the candidates and choose the most appropriate ones. At the end of this first stage, node A has the list of all the nodes that it is covering, and their respective metrics. In addition, each of the covered nodes knows which node will be its parent node.

\subsubsection{Children Selection Process}

Just after having sent the Hello Message, the Active Candidate node changes its state to Waiting Candidate and sets a timeout to receive the replies from its neighbors. Ev- 
ery time a Parent Recognition Message is received, the selection metric is stored in a list of candidates.

If a Waiting Candidate node does not receive any Parent Recognition Messages from its neighbors, it turns off because there are no new nodes to cover on its neighborhood. This is the case of nodes E and B in the final topology shown in Figure 4.1(g), given that they have no children. If at least one node replies to the Hello Message, the Waiting Candidate will change its state to Active Node because it is covering at least one node in the topology.

Once the timeout expires, the parent node changes its status to Active Node and sorts the list in decreasing order according to the selection metric. The parent node then sends a Children Recognition Message that includes the complete sorted list of candidates to all its dominated nodes. In Figure 4.1(c), node A broadcasts the sorted list to nodes B, C, D, and $\mathrm{E}$.

After the children nodes receive the list, they change their status to Candidate node and set a timeout proportional to their position in the candidate list. During that timeout the candidates wait for Sleeping Messages coming from their "brother" nodes. If a node receives a Sleeping Message during the timeout period, it turns itself off for a certain period of time and changes its status to Sleeping Candidate, meaning that one of its brothers is better qualified to become part of the tree. Based on this scheme, the best node according to the metric sends a Sleeping Message first, blocking any other candidate brother in its range. Therefore, only the other candidate nodes outside its area of communication have the opportunity to start their own generation process. For example, in Figure 4.1(d), node D received a Sleeping Message from E before its timer expired, so it turned itself off. A Candidate Node that does not receive any Sleeping Message changes 
its state to Active Candidate and starts its own process of looking for unvisited nodes by sending a Hello Message, as in 4.2.1.

\subsubsection{Second Opportunity Process}

Although the first two stages are sufficient in most scenarios to produce a connected reduced topology, there are some cases in which this does not happen. One example is the case of node D in Figure 4.1(d), which is sent to sleep by node E, because E sends the Sleeping Message first. If node D never wakes up, node $\mathrm{G}$ will not receive a Hello Message from any other node so it will not participate in the tree creation process. This case will be the same for all nodes sent to sleep which have a bottleneck link in the graph. In order to avoid this situation, when a node receives a Sleeping Message, instead of going directly to the Sleeping Node mode, it sets a timer that, once expired, the node turns back on, changes its status to Active Candidate and sends a Hello Message to start its own neighborhood discovery process, as seen in Figure 4.1(e). In Figure 4.1(f), node D becomes part of the CDS after going through the Second Opportunity Process, because it discovers node $\mathrm{G}$ and goes to the Active Node mode. This operation increases the overhead of the algorithm, but guarantees connectivity of all the nodes in the graph, as proved in Lemma 1.

LEMMA 1. IF THE INITIAL GRAPH IS CONNECTED, THE REDUCED GRAPH IS ALSO CONNECTED.

Proof: Assume that a connected original graph is given. If at the final stage of the algorithm there exists at least one node that has not been covered, it is because it did not receive any Hello Message. Given that every node is forced to send a Hello Message, each node explores all its neighbors from the original graph looking for unvisited nodes. The 
algorithm continues until all nodes are visited in the total area of coverage of the tree, exploring all edges from this set of nodes. This means that the final tree is a connected subgraph itself because it has no edge to unvisited nodes. If an uncovered node exists at the end of the execution of A3, it means that there is no edge between any of the covered nodes and the uncovered node. Then the initial graph cannot be connected because it has at least two non-connected sets of nodes, which contradicts the initial assumption of a connected graph.

It is worth emphasizing that A3 is completely distributed and needs no synchronization scheme nor localization information. The process finishes when the last node finishes its own creation process. Each node is responsible for its own process and needs no information about the status of the overall process. Actually, as soon as a node is selected as part of the CDS tree, it can start its application-related tasks. Figure 4.2.1 shows the finite state machine of a node running the A3 protocol. As it can be seen, the A3 algorithm guarantees that, once the algorithm is run, every node is in either Active Node or Sleeping Node state.

The computational complexity of the A3 algorithm can be easily calculated based on the fact that the sorting function executed by the parent nodes is the most expensive operation. Therefore, the complexity of the algorithm is given by the complexity of the sorting routine, which can be bounded as $O(n \log n)$. The message complexity is bounded by the worst case of 4 messages in the case of a node that becomes a parent node at the first opportunity: Hello, Parent Recognition, Children Recognition, and Sleeping messages. Therefore, the message complexity is $O(n)$ for a network with $n$ nodes, with a worst case scenario of $4 n$ messages. 


\subsubsection{The Selection Metric}

Several metrics have been utilized in the literature to select the active nodes, such as the distance between the nodes, the node degree of the candidate nodes, and the potential coverage of the candidate nodes, being among the most important ones. For example, in the the work of [41], the authors use the amount of unvisited nodes that each candidate neighbor would provide to the discovered topology. However, these metrics usually have two main drawbacks. First, they do not consider the amount of residual energy in the nodes, so they could select nodes that might die soon. Second, the geometry of the topology is also ignored, which could drive to a non appropriate selection of nodes. For example, if all candidates include the same amount of nodes, probably the ID will be used as a tiebreaker, which may devolve into the selection of nodes that interfere with other active neighbors. Finally, these metrics usually require sending messages to nodes that are two hops away, increasing the overhead considerably.

The selection metric utilized by the A3 algorithm is simple, requires only one hop information, and also considers the residual energy of the nodes. Nodes only need to measure the Received Signal Strength Indicator (RSSI) of the Hello Message sent from their dominant nodes. Then, using their residual energy, the final metric is calculated using Equation 4.1, which is a convex combination of the residual energy in the child node and its distance to the parent node. The metric, sent back to the parent node in the Parent Recognition Message, is calculated as follows:

$$
M x, y=W_{E} \cdot \frac{E_{x}}{E_{\max }}+W_{D} \cdot\left(\frac{R S S I_{y}}{R S S I^{*}}\right)
$$

where $x$ is the candidate node, $y$ is its parent node, $W_{E}$ is the weight for the remaining energy in the node, $E_{x}$ is the remaining energy in node $x, E_{\max }$ is the maximum initial en- 
ergy, $W_{D}$ is the weight for the distance from the parent node, $R S S I_{y}$ is the received signal strength from the parent node, and $R_{S S I}^{*}$ is the minimum RSSI to ensure connectivity, which is given by the sensitivity of the receiver. Note that, given that $W_{E}+W_{D}=1$, Equation 4.1 produces a value between 0 and 1 that is assigned to each neighbor when added to the list; the higher the value of the metric, the higher a priority the node will receive in the selection process, increasing its probability to be active in the tree.

As it can be seen from Equation 4.1, the selection metric gives priority to those nodes with higher energy and which are farther away from the parent node. The final effect of this choice is to have a tree with fewer nodes and better coverage. However, proper weight manipulation can satisfy different criteria, as needed by the network operator. If communication coverage is to be optimized and the average height of the node in the tree (number of hops) needs to be reduced, the distance metric must be weighed more heavily. The downside is that low energy nodes may be included in the tree, which may introduce early failures of nodes, reducing reliability; and may increase the number of calls of maintenance processes, therefore reducing the lifetime of the tree. On the other hand, if reliability of the tree is desired, energy must be weighed more. The downside is that the tree may present more active nodes, due to the fact that the geometric distribution of the nodes is not taken into account. In this dissertation, a balanced average with both weights set to 0.5 , is used in all performance evaluations. Further analysis of the impact of the weights in the performance of the protocols is part of the future work after this dissertation. 


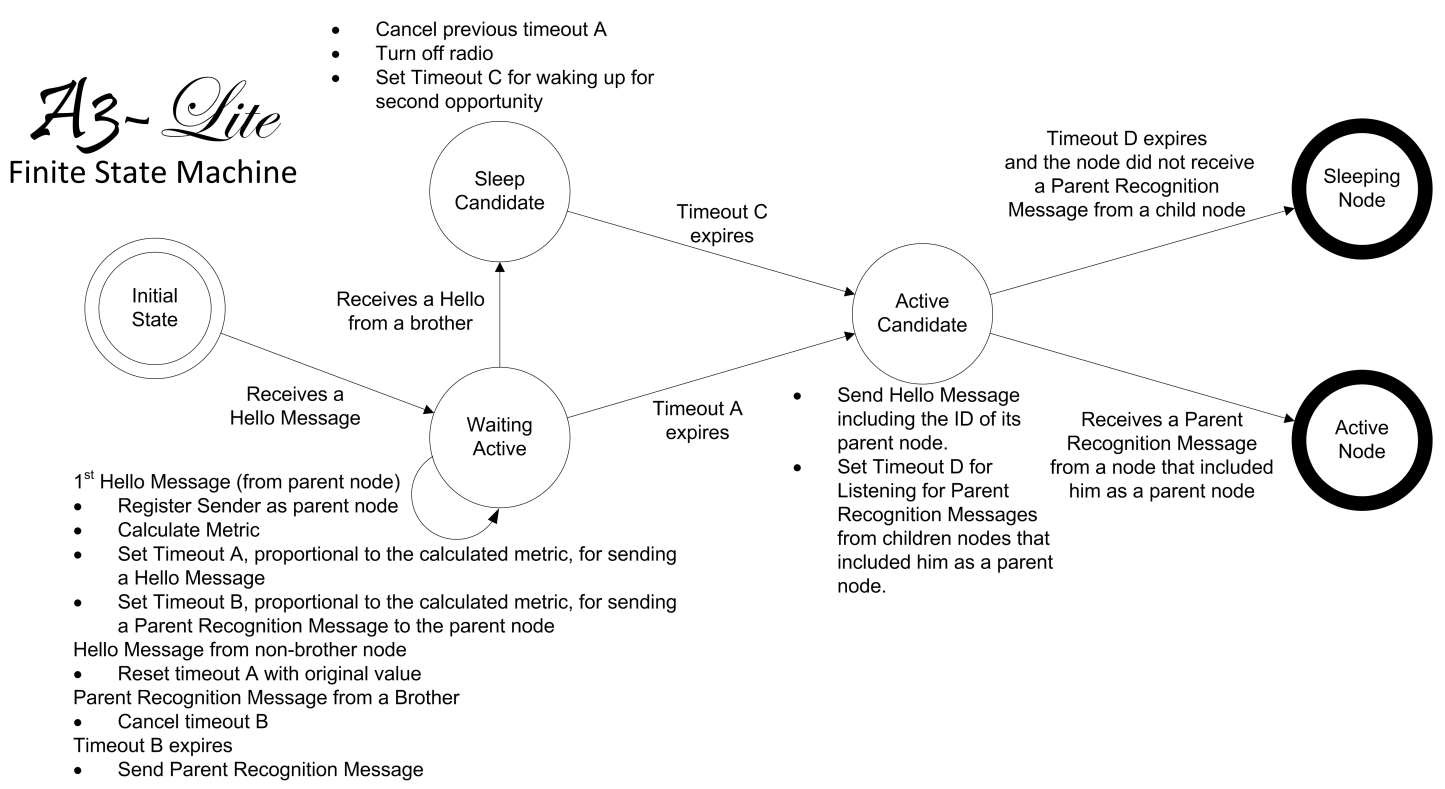

Figure 4.3: The A3Lite protocol finite state machine.

\subsection{The A3Lite Algorithm}

Even though A3 is a very simple protocol and offers a very low message and computational complexity, it can be made even simpler by relaxing the need of a centralized selection process in the parent nodes in order to determine the priority of its candidate neighbors. This is the idea behind A3Lite, which only needs a total of $2 n$ messages to create a tree similar or better to the one created by A3.

\subsubsection{The Neighborhood Discovery Process}

Similar to A3, a preselected node, let us say node $\mathrm{X}$, starts the tree creation process by sending a Hello Message. Each Hello Message includes the ID of the parent node, except in the case of the sink which in that field will have its own ID. When the neighbors of node $\mathrm{X}$ receive the message, they change their status to Waiting Active, calculate the 
selection metric of A3 (Equation 4.1) in the same manner, and register the sender as its parent node. Then, each node sets timeout A inversely proportional to their selection metric, $t_{0}+\left((1-\right.$ metric $\left.) \cdot t_{1}\right)$, where $t_{0}<<t_{1}$ to send a Hello Message and timeout $\mathrm{B}$, set to half the value of timeout A, to send a Parent Recognition Message.

\subsubsection{Children Selection Process}

At this point, during the Waiting Active state several events may happen:

- The node may receive a Parent Recognition Message from one of its brothers (all nodes under the area of coverage of the same parent node). In this case, the receiver node cancels timeout $\mathrm{B}$ and remains in the Waiting Active state.

- The node may receive a Hello Message from a non-brother, in which case the node resets timeout A to its original value and remains in the Waiting Active state. This means that a non-brother node started a neighborhood discovery process and needs some time to explore and let its branch grow to cover as many nodes as possible, decreasing the probability of the receiver node having unvisited nodes in its neighborhood and becoming active.

- Timeout A may expire. When this happens, the node sends a Hello Message and goes to the Active Candidate state.

- Timeout B may expire. In this case, the node sends a Parent Recognition Message and remains in the Waiting Active state.

- The node may receive a Hello Message from a brother, in which case the node goes to the Sleep Candidate state. Upon reaching this state, the node turns the radio off temporarily, cancels timeout $\mathrm{A}$ and sets timeout $\mathrm{C}$, equal to $t_{2}+((1-$ metric $)$. 
$\left.t_{1}\right)$, where $t_{0}<<t_{2} \leq t_{1}$. When timeout $\mathrm{C}$ expires, the node will wake up and start its Second Opportunity Process, in which the node goes to the Active Candidate state to explore its neighborhood for unvisited nodes.

Once the node is in the Active Candidate state, it sends a Hello Message and sets timeout $\mathrm{D}$, equal to $t_{3}$, where $t_{1}<t_{3}$, in order to wait for Parent Recognition Message from its children. If timeout $\mathrm{D}$ expires and no message has been received, the node goes to the Sleeping Node state. If the Active Candidate receives at least one Parent Recognition Message from a child, then the node goes to the Active Node state, which means, that it will be part of the CDS tree.

Compared to A3, A3Lite requires only a maximum of two messages per node, which is at most half the message complexity of its predecessor. Also, as it can be seen in Figure 4.3 that depicts the finite state machine of the A3Lite algorithm, A3Lite keeps the connectivity property from A3 described in Lemma 1, given that in A3Lite every node sends a Hello Message with the address of its parent node, so if any unvisited node existed at the end of the execution it is because it was not connected in the original graph.

\subsection{Performance Evaluation}

In this section the results of the performance evaluation comparing the A3 and A3Lite algorithms with the EECDS and the CDS-Rule-K heuristics algorithms and with the MIPMCDS optimal solutions are presented.

Three sets of experiments are included. The first set maintains the number of nodes fixed and increases the node degree by changing the communication range of the nodes. The communication ranges used in the experiment are proportional to the critical transmission 
Table 4.1: Simulation parameters for connectivity-oriented protocols.

\begin{tabular}{|c|c|c|c|}
\hline & Experiment 1 & Experiment 2 & Experiment 3 \\
\hline $\begin{array}{l}\text { Deployment } \\
\text { area }\end{array}$ & \multicolumn{3}{|c|}{$200 \mathrm{~m} \times 200 \mathrm{~m}$} \\
\hline $\begin{array}{l}\text { Number of } \\
\text { nodes }\end{array}$ & 100 & $\begin{array}{l}10,20,40,60, \\
80 \text { and } 100\end{array}$ & 50 and 101 \\
\hline $\begin{array}{l}\text { Transmission } \\
\text { Range - } \\
\text { Distances } \\
\text { based on RSSI }\end{array}$ & $\begin{array}{l}1,1.5,2 \text { and } \\
3 \times C T R(100) \\
\text { equivalent } \\
\text { to: } 27 \mathrm{~m}, \\
41 \mathrm{~m}, 54 \mathrm{~m}, \\
68 \mathrm{~m} \text { and } 81 \mathrm{~m} \\
\text { (Eqn. } 2.1 \text { ) }\end{array}$ & $\begin{array}{l}60 \mathrm{~m} \\
\text { equivalent } \\
\text { to } 1 \mathrm{xCTR}(10)\end{array}$ & $30 \mathrm{~m}$ \\
\hline $\begin{array}{l}\text { Node } \\
\text { Distribution }\end{array}$ & $\begin{array}{l}\text { Uniform } \\
(200,200)\end{array}$ & $\begin{array}{l}\text { Uniform } \\
(200,200)\end{array}$ & $\begin{array}{l}\text { Grid HV and } \\
\text { Grid HVD }\end{array}$ \\
\hline $\begin{array}{l}\text { Instances per } \\
\text { topology }\end{array}$ & \multicolumn{3}{|c|}{50 instances } \\
\hline$E_{\max }$ & \multicolumn{3}{|c|}{1 Joule } \\
\hline A3 Weights & \multicolumn{3}{|c|}{$W_{E}=0.5, W_{D}=0.5$} \\
\hline $\begin{array}{l}\text { Energy } \\
\text { Consumption }\end{array}$ & \multicolumn{3}{|c|}{$\begin{array}{c}\text { Eelec }=50 \mathrm{~nJ} / \text { bit } ; \text { Eamp }=10 \mathrm{pJ} / \mathrm{bit} / \mathrm{m}^{2} \\
\text { Short Messages }=25 \text { Bytes } \\
\text { Hello, Parent Recognition and Sleeping Messages } \\
\text { Long Messages }=100 \text { Bytes } \\
\text { Children Recognition and Data messages } \\
\text { Idle state energy consumption assumed negligible }\end{array}$} \\
\hline
\end{tabular}

range (CTR) formula in Equation 2.1 presented in Section 2.2.1.1, calculated based on the defined network size and the area of deployment.

The second set, on the other hand, varies the network density by changing the number of nodes while maintaining a fixed communication range. In these two experiments, the nodes are uniformly distributed in the area of deployment.

The third set of experiments includes an additional theoretical comparison considering an ideal grid topology in which all nodes have the same number of neighbors. Two different grid topologies are used: the Grid HV topology, in which each node can listen to its horizontal and vertical neighbors; and the Grid HVD topology, in which each node can listen 
to its horizontal, vertical, and diagonal neighbors. Therefore, the number of neighbors in those topologies is at most 4 and 8 , respectively. Figure 4.6 shows these scenarios. All of the experiments show the average result of 50 random scenarios for the first two experiments, and 50 replicates of the grid topologies for the third experiment.

Three performance metrics are utilized to assess the performance of the topology construction algorithms: 1) number of active nodes; 2) number of messages used in the CDS building process; and 3) amount of energy used in the process. The first metric shows how the topology construction mechanism can effectively reduce the amount of active nodes while preserving network connectivity. The other two metrics show how efficient the algorithm is in terms of overhead and energy consumption.

The algorithms are evaluated in scenarios that go from sparse to dense topologies, and from low to high node degree. The node degree and the density of the network are modified by increasing the communication range of the nodes and the number of nodes in the network. The four algorithms were implemented in a Java-based event-driven simulation tool called Atarraya [107], designed for the purpose of testing topology control algorithms. More details on the simulation tool can be found in Appendix A. Table 4.1 presents a summary of the simulation variables used in the experiments.

\subsubsection{Experiment 1: Changing the Node Degree}

The main goal of this experiment is to compare the algorithms when the node degree of the network is changed by increasing the transmission range of the nodes. Given that these algorithms work based on information from neighbors, it is important to measure their performance with neighborhoods of different sizes. 


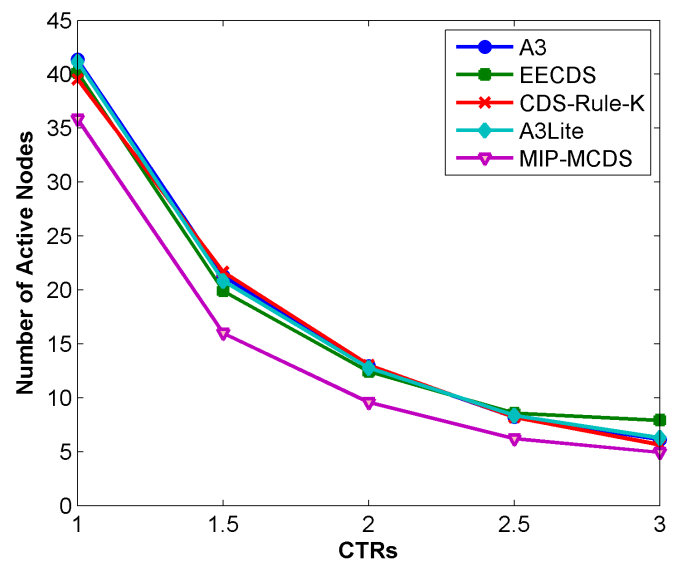

(a) Number of actives nodes.

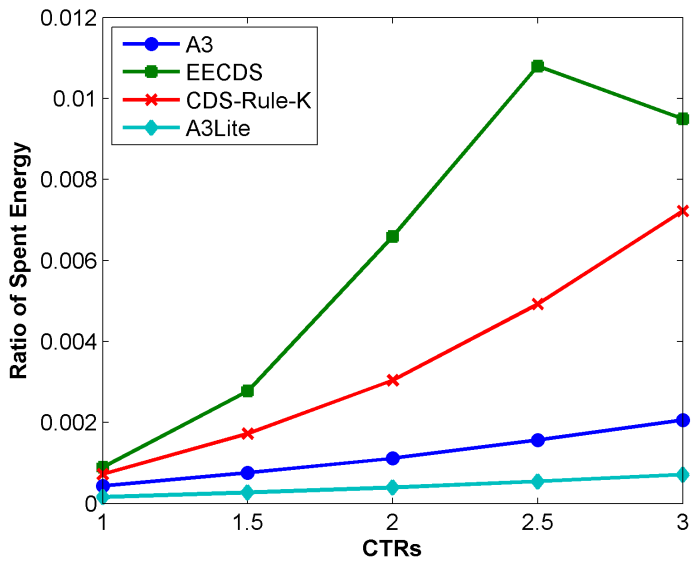

(b) Energy used in the CDS creation.

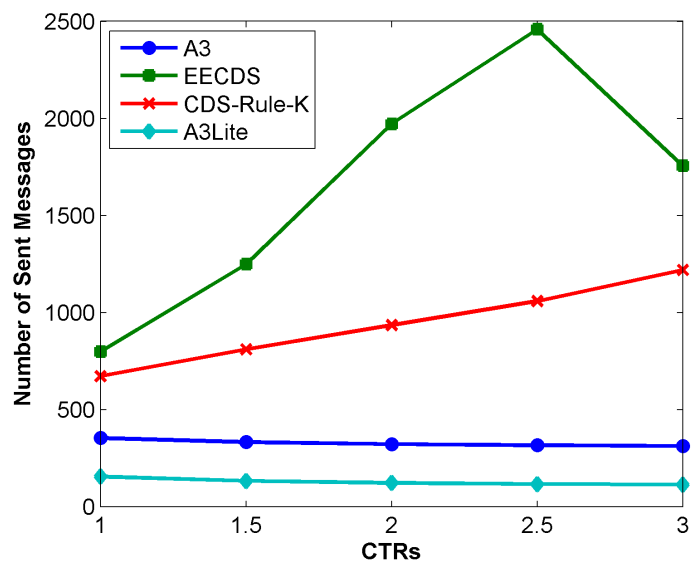

(c) Number of messages sent.

Figure 4.4: Results of experiment 1: changing the node degree. 
As it can be seen from Figure 4.4(a), the three algorithms produce a similar number of active nodes. A3 and EECDS produce almost the same number of active nodes and CDSRule-K produces the largest reduced topologies. The trend in all the algorithms is to decrease the number of active nodes with the node degree, as expected, given that with larger communication ranges, each node covers more area, and thus more nodes, so less active nodes are required to cover the whole network. The gap between the optimal solution and the approximation algorithms starts at five nodes and then decreases, when the communication range and the node degree increase, to an almost one node difference. This result seems to imply that the performance of the approximation algorithms tend to provide reduced topologies very close to the optimal when the node degree density is high. This behavior can be explained by the fact that having larger communication ranges and larger sets of nodes to choose from, the selection criteria of the nodes in the approximation algorithms does not have much impact while still practically covering all nodes of the deployment area; in other words, the optimal number of active nodes would very hard to miss.

Figures 4.4(b) and 4.4(c) show two important metrics: the total energy and number of messages used to build the CDS trees, respectively. In this case, the A3 and A3Lite protocols show their superior performance, with A3Lite having the least amount of sent messages and energy used in the topology construction process. The A3 and A3Lite protocols present a slightly-increasing linear energy consumption when compared with the CDSRule-K and EECDS algorithms, which show a very fast increase rate.

The fast increasing use of energy of the CDS-Rule-K protocol is explained by its pruning process in which every node must update nodes two hops away when it is unmarked. The overhead of this notification increases with the number of neighbors because more 
nodes will retransmit the message. Also, when the node degree increases, more nodes get unmarked and will produce this extra overhead.

In the case of the EECDS algorithm, the factor that increases the amount of messages (and energy, consequently) is related to the competition used in both phases of the algorithm. This is due to the fact that with a higher communication range, more nodes are covered, and the tree has fewer nodes in higher levels. This, at the same time, reduces the amount of nodes competing to become part of the tree in the outer regions of the topology. However, EECDS shows a reduction in networks with high node degree, which means that it benefits from having large communication ranges because it may avoid having to calculate many levels in a tall tree, which always involves a great number of messages.

The linearity of the A3 and A3Lite protocols is a consequence of the bounded number of messages that each node needs to transmit, which remains almost identical and never goes over $4 n$ and $2 n$ in ideal conditions, respectively. Furthermore, the number of messages in the A3 and A3Lite protocols decreases when the density is higher; this fact can be explained by the fact that less nodes are required to provide connectivity in the network, so more nodes will be turned off, and they will not send any more messages. In the case of the energy, the increasing behavior is due to impact of overhearing messages from neighbors nodes, where the neighborhoods grow with the node degree.

\subsubsection{Experiment 2: Changing the Node Density}

The main goal of this experiment is to compare the results produced by the algorithms when the network density is changed by varying the number of nodes in the area while keeping a fixed communication range of $60 \mathrm{~m}$, equivalent in this experiment to $1 x C T R$ of 


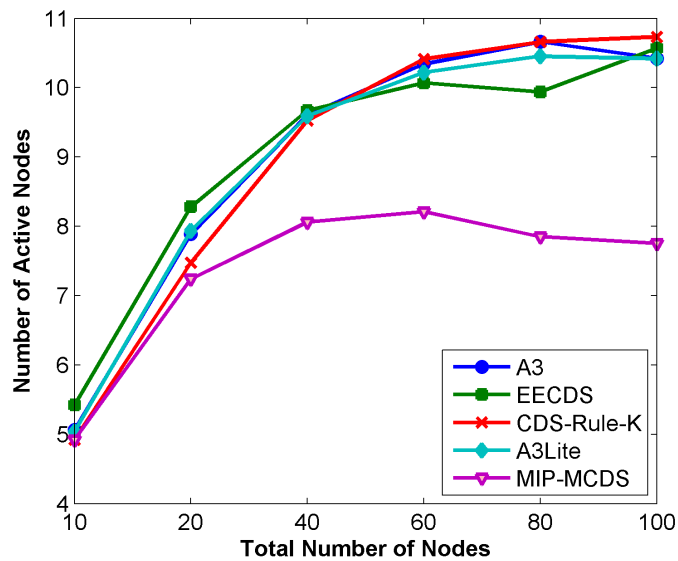

(a) Number of actives nodes.

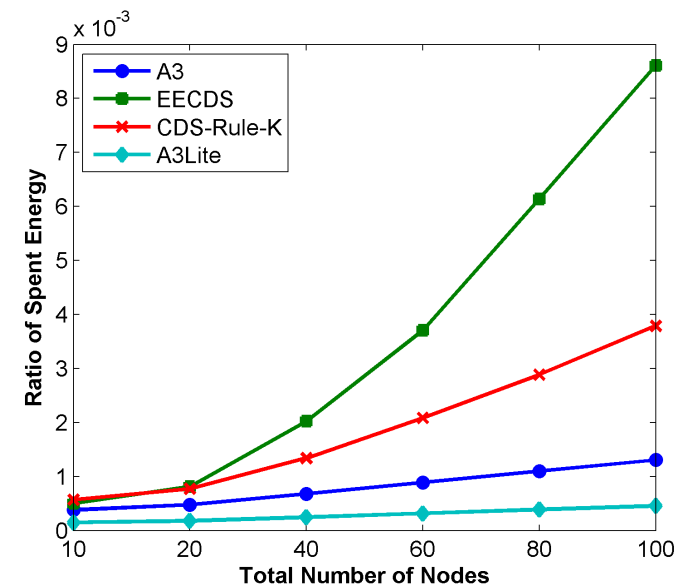

(b) Energy used in the CDS creation.

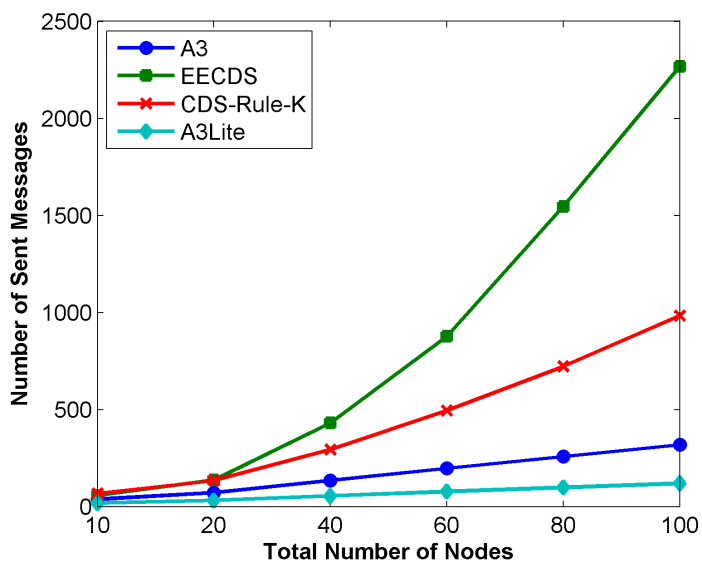

(c) Number of messages sent.

Figure 4.5: Results of experiment 2: changing the node density. 
10 nodes. This experiment is important to show how scalable the algorithms are in dense topologies and how the resource usage depends on the number of nodes.

The results, shown in Figure 4.5, show similar results in terms of energy and messages, but a slightly different scenario, as seen in Figure 4.5(a). In this case, the CDS-Rule-K shows the best results in the sparse networks up to scenarios with 40 nodes, with some advantage of this protocol in small topologies. From 60 nodes and above, it produces a similar or worse number of actives nodes compare to A3. The A3 and A3Lite protocols show a very similar behavior in the different scenarios. EECDS appears to be have a good performance in dense networks; however, the difference in active nodes is less than one active node, so it can be easily assumed that they tend to produce the same value. Compared to the optimal solution, the approximation algorithms produce reduced topologies that are very close to the optimal in small networks, and then start to show a greater advantage when the size of the network increases. It can be seen that the optimal solution converges rapidly to an average value of seven to eight active nodes for the scenarios shown, despite the number of nodes. The convergence is shown also by the approximation algorithms, but it is between ten and eleven nodes, which represents an average of $35 \%$ error from the approximate solutions to the optimal, with a standard deviation of 0.19 for A3. This high variation could be appreciated when inspecting the results of individual topologies with 100 nodes, in which A3 and A3Lite could obtain the optimal solution in a few cases, while in other topologies the approximate solutions included twice the amount of active nodes in the optimal solution.

In terms of the message complexity and energy efficiency, the CDS-Rule-K presents an almost exponential increase, the EECDS a fast linear increase, and the A3s show low and linearly bounded numbers of messages and energy consumption, as shown in Fig- 

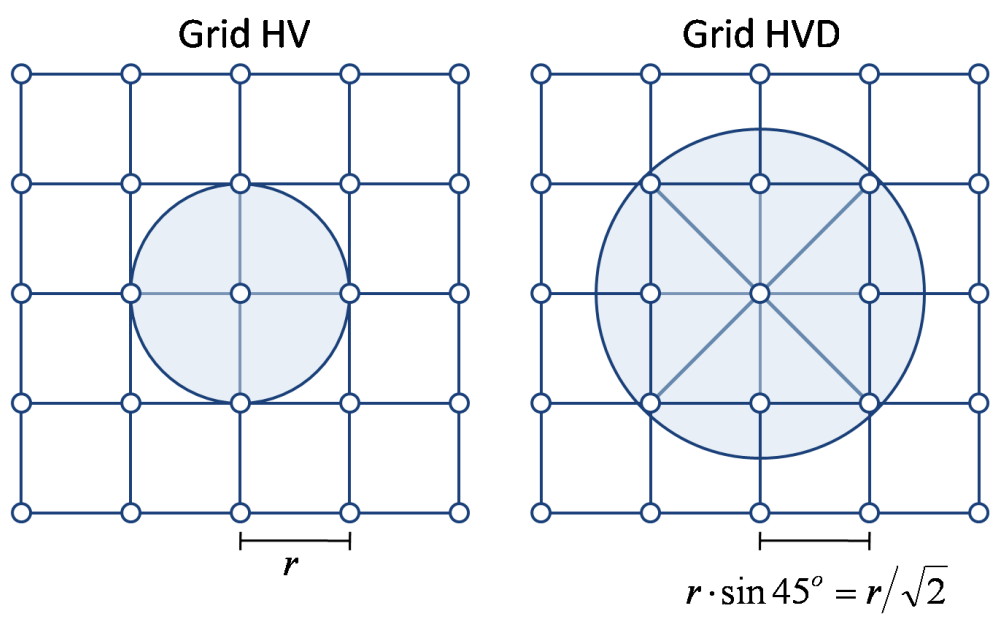

Figure 4.6: Square grids deployment.

ures 4.5(b) and 4.5(c). This shows that the A3 and A3Lite algorithms are scalable and are not highly affected by the number of nodes deployed or the node degree in the network.

\subsubsection{Experiment 3: Ideal Grid Topologies}

The third experiment considers the ideal grid scenario with its two variants: HV and HVD, as shown in Figure 4.6. This experiment shows the performance of the algorithms in a perfectly homogeneous topology, with ideal conditions of density and node degree, which could be considered a predefined scenario such as a sensor network deployed in an office building. From Figure 4.7, it can be seen that the A3 and A3Lite algorithm show similarity in the number of actives nodes, with A3Lite selecting 54\% of the nodes in the grid $\mathrm{HV}$ and $31 \%$ in the grid HVD scenarios to be active, versus 58\% and 33\% from A3, $57 \%$ and $31 \%$ from EECDS, $58 \%$ and $32 \%$ from CDS-Rule-K algorithms, and $42 \%$ and $25 \%$ from the MIP-MCDS solutions. This means that the approximation protocols were at least $12 \%$ from the optimal in the HV grid, and around 6\% in the HVD grid. The other 
two metrics show an increasing trend for EECDS and CDS-Rule-K while A3 and A3Lite show a bounded cost in overhead and energy. 


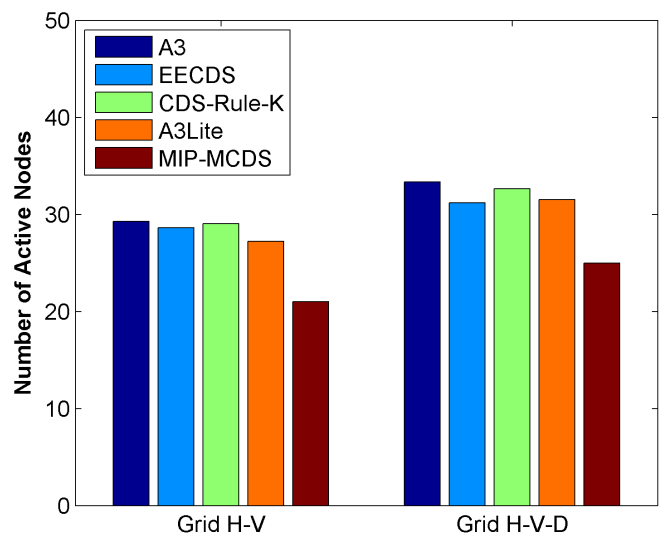

(a) Number of actives nodes.

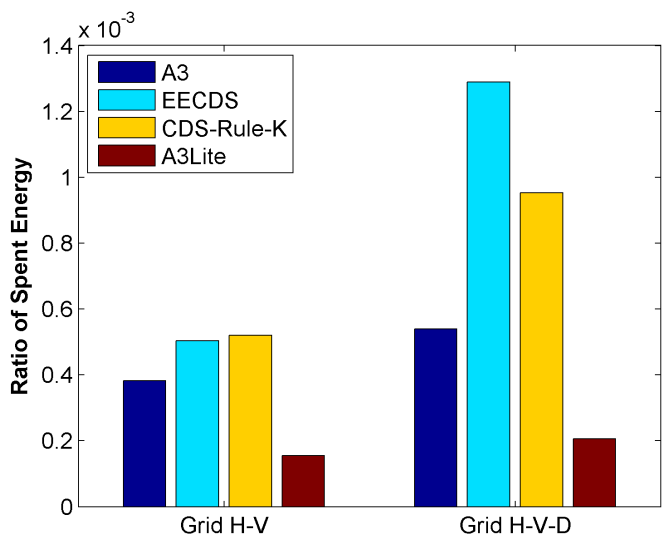

(b) Energy used in the CDS creation.

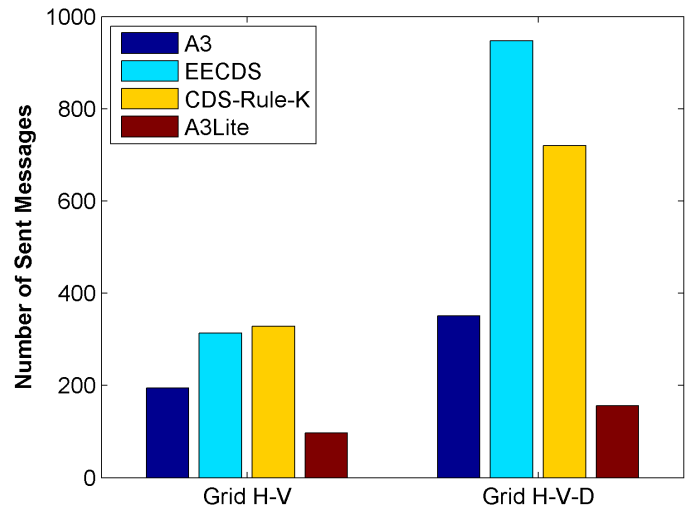

(c) Number of messages sent.

Figure 4.7: Results of experiment 3: ideal grid topologies. 


\section{Chapter 5: The A3Cov and A3CovLite Topology Construction Protocols for Coverage}

The protocols presented in Chapter 4 are intended to build a connected topology with the minimum amount of active nodes; however, as it was discussed before, connectivity is not the only desired feature in topology construction protocols. The sensing coverage is a very critical factor that determines the success of a protocol because it guarantees that most of the area of interest will be monitored by the network. The two problems can be assumed similar when $R_{\text {Comm }}=R_{\text {Sense }}$, in which case the connectivity-oriented protocols usually offer a good coverage ratio, but this is not a very realistic assumption to make. In this section two new protocols will be introduced, as extensions of the A3 and A3Lite algorithms described before: the $\mathrm{A} 3 \mathrm{Cov}$ and $\mathrm{A} 3 \mathrm{CoveLite} \mathrm{protocols.} \mathrm{The} \mathrm{main} \mathrm{idea} \mathrm{be-}$ hind the new protocols is to use the connected structure produced by the original algorithms as a starting point and then include more nodes that will extend the coverage ratio of the area of interest. The $\mathrm{A} 3 \mathrm{Cov}$ and $\mathrm{A} 3 \mathrm{CovLite}$ protocols, as in the original version, do not need any location information, work in a distributed manner and also work in

scenarios where $R_{\text {Comm }} \neq R_{\text {Sense }}$, in comparison with many coverage-oriented protocols which can only guarantee connectivity when $R_{\text {Comm }} \geq 2 R_{\text {Sense }}$. The new protocols will be compared against their predecessors in order to show the considerable gain in coverage. In addition, they will be compared with the theoretical optimal 
grid deployments from $[69,88]$ and also, with two other coverage-oriented protocols ACOS and StanGA, both described previously in Section 2.3.3.

\subsection{The A3Cov Algorithm}

From the description of the original A3 algorithm it is known that A3 sends to sleep all nodes in the reduced tree topology that are under the communication range of their corespondent parent nodes. While this technique may work just fine in the case where the communication and sensing radii are equal, it may not provide satisfactory results when the sensing radius is smaller than the communication radius; in other words, many nodes will be put to sleep because they are reachable via communication range but the area where they are may not be covered sufficiently by their sensors.

Therefore, if sensing-coverage is to be increased, more nodes would have to be selected to remain active. One question comes to mind here: which non-CDS nodes should the protocol add to the active subset so that coverage is substantially increased without including too many extra active nodes? In the first place, and given the lack of location information, the generation of an accurate geometric map of coverage can be very expensive in both message and computation complexity. This is the main reason why both $\mathrm{A} 3 \mathrm{Cov}$ and A3CovLite work based on the statement presented in [71], where the authors propose that, in a dense network, the coverage of the nodes' locations is a good approximation of the coverage of the complete area of interest. This means that a similar approach to the one adopted for connectivity can be also applied for coverage, and that is the foundation of $\mathrm{A} 3 \mathrm{Cov}$ and $\mathrm{A} 3 \mathrm{CovLite.}$

Focusing on the description of the protocols, $\mathrm{A} 3 \mathrm{Cov}$ inherits most of the $\mathrm{A} 3$ protocol in order to obtain an initial connected topology. The main difference between the proto- 


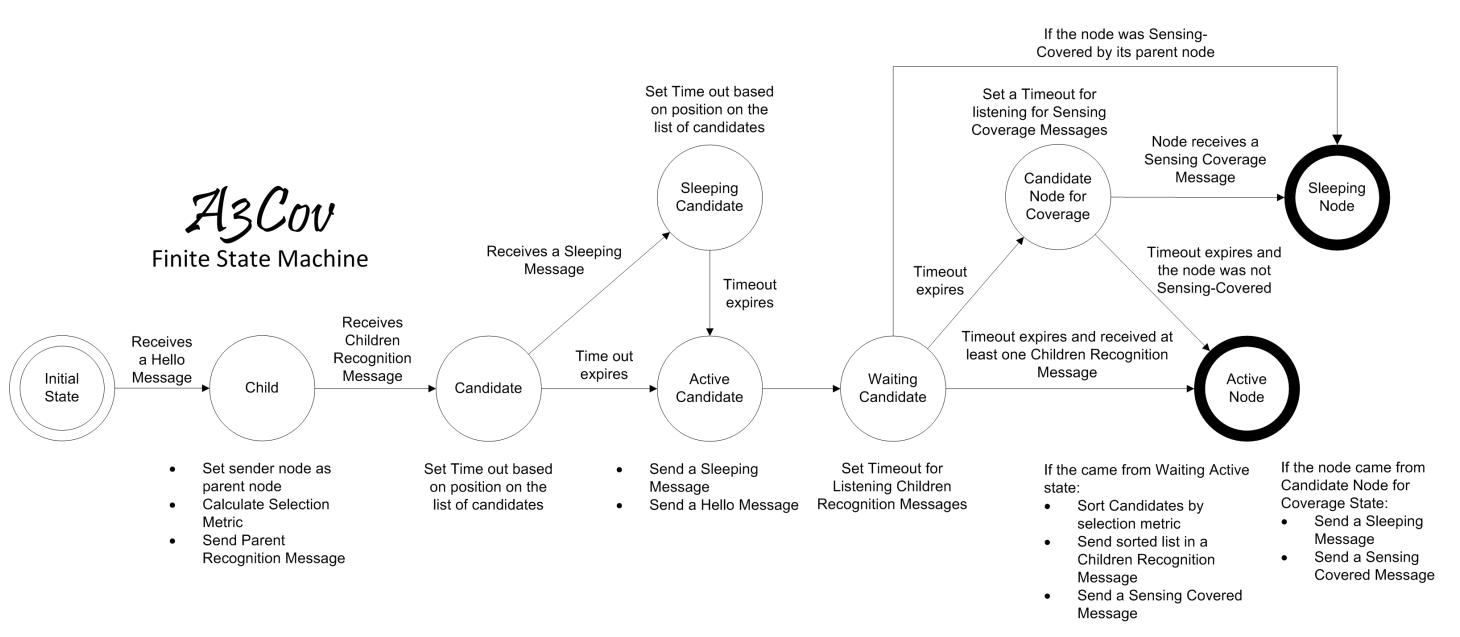

Figure 5.1: The A3Cov protocol finite state machine.

cols is that $\mathrm{A} 3 \mathrm{Cov}$ applies two different selection metrics, one for connectivity and one for coverage. The first one was maintained from the A3 protocol because it produces a connected reduced topology which favors the selection of neighbor nodes that are farther away from the parent node, decreasing as much as possible the overlapped sensing area between them. The second selection policy keep some of the leaf nodes active in order to extend the coverage provided by the communication backbone alone. This selection policy is based on the idea that if a node is inside the sensing range of an active node it will not be eligible to remain active, unless it is required for connectivity purposes.

Assuming the sensing range as a perfect disc with radius $R_{\text {Sense }}$ and centered at the node's position, if the distance between an active node and a regular node is less that $R_{\text {Sense }}$, then the regular node is considered sensing-covered. This criterion is evaluated twice during the execution of the protocol: when the node receives the Children Recognition Message from its parent node, in which it tests if it has been sensing-covered by the parent node, and when a node receives a Sensing Coverage Message from any neighbor. The Sensing Coverage Message can arrive at any moment during the execution of the protocol and, if 
the sensing coverage criteria is accomplished, that is, if $d(x, y) \leq R_{\text {Sense }}$, then the receiver is said to be sensing-covered by the sender.

A node can be selected to become active for coverage purposes just after the node was in the Waiting Candidate state and have sent a Hello Message with no answer from unvisited neighbor nodes. At this moment, the original A3 would would have sent all those nodes to the Sleeping Node state, while A3Cov verifies first if the nodes have been sensing-covered by another active node, in which case they are sent directly to the Sleeping Node state.

The Waiting Candidate nodes that were not sensing-covered by their parent nodes and that lack any children nodes, become Candidate Nodes for Coverage and are asked to stay awake for an extra period of time in order to listen for Sensing Coverage Message from their neighbors. The new timeout used by these nodes to wait for the Sensing Coverage Message is also proportional to the metric used for connectivity, which considers distance and energy, so both selection processes share the same priority policy.

If a Candidate Node for Coverage receives a Sensing Coverage Message before the timeout expires, and $x, y \in V, d(x, y)<R_{\text {Sense }}$, the node is considered sensing-covered, and goes to the Sleeping Node state immediately. If $d(x, y)>R_{\text {Sense }}$ it means that the receiver node is under the communication coverage of the sender but not within its sensing coverage, so the message is ignored and the receiver node keeps waiting for messages until the timeout expires.

When the timeout expires, if the node has not been sensing-covered, i.e., it has not received any Sensing Coverage Messages, the node remains active, changes its state to Active Node, and sends a Sensing Coverage Message to its neighbors in order to notify its new state. In addition, each Active Node broadcasts a Sleeping Message in order to delay the neighborhood discovery process of its neighbor nodes and decrease the proba- 


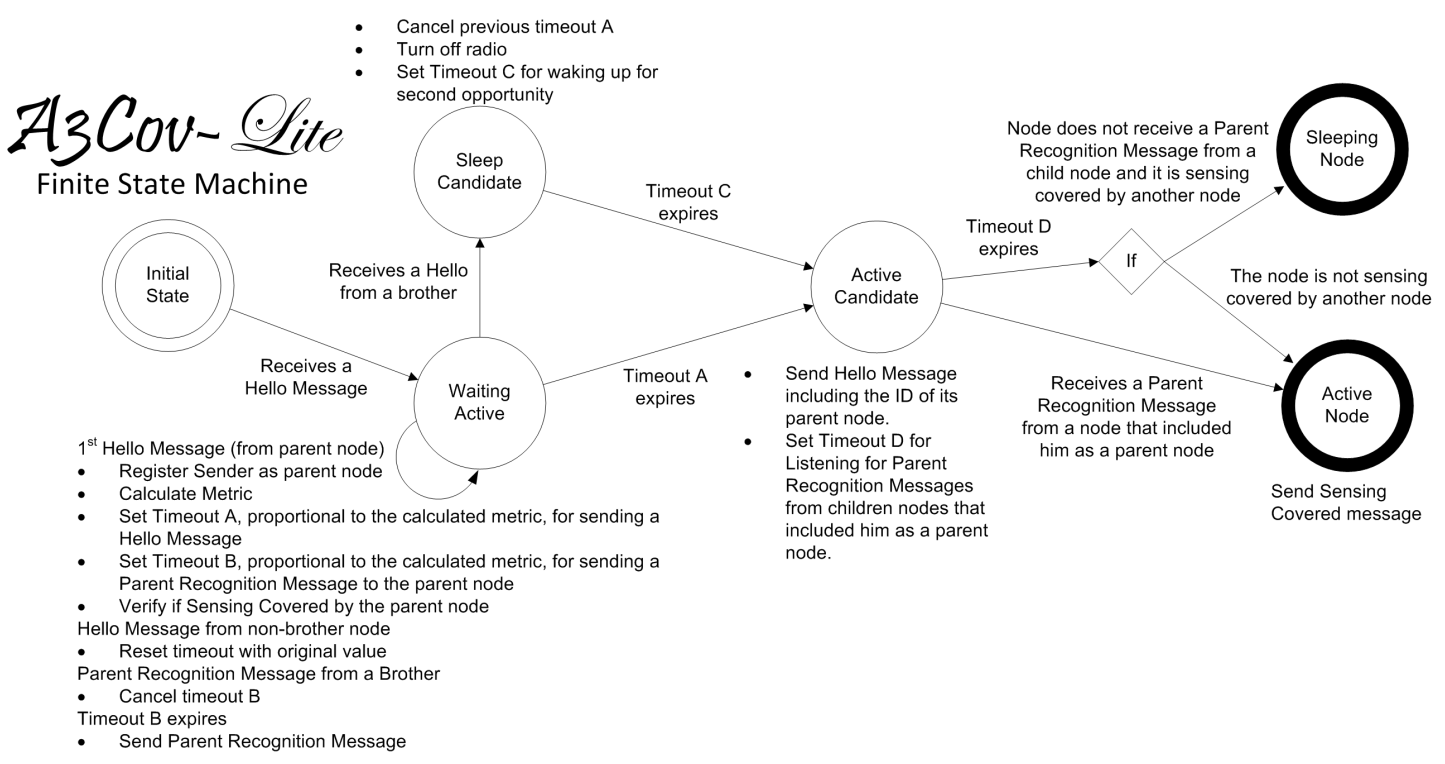

Figure 5.2: The A3CovLite protocol finite state machine.

bility of having too many active nodes in its neighborhood. The nodes who were selected in the first place as part of the CDS tree (active nodes) for connectivity purposes, are asked to broadcast a Sensing Coverage Message also in order to update their neighbors' sensing-coverage status.

As it will be shown later in the performance results, the coverage-based protocols improve the coverage in those cases where $R_{\text {Comm }} \neq R_{\text {Sense }}$, compared to their predecessors. Figure 5.1 depicts the finite state machine of the $\mathrm{A} 3 \mathrm{Cov}$ algorithm. A3Cov has the same computational complexity of A3 and the same message complexity $O(n)$, only that each node may send up to five messages $(5 n)$. In addition, the proof of Lemma 1 is also valid for $\mathrm{A} 3 \mathrm{Cov}$ due to the fact that every tree produced by $\mathrm{A} 3 \mathrm{Cov}$ contains an underlying tree from A3 with extra nodes added for coverage purposes, so connectivity is not affected. 


\subsection{The A3CovLite Algorithm}

A3CovLite is the respective extension of A3Lite in order to provide better coverage of the area. A3CovLite works exactly like A3Lite, except for the following two moments: when a node receives the first Hello Message and when timeout D expires for a node in Active Candidate state. In the first case, when an unvisited node received a Hello Message, it evaluates if it is sensing-covered by its parent node. In the second case, timeout D is set to listen for Parent Recognition Messages from children nodes. If the node does not receive any messages of this kind, it means that it does not provide connectivity to any node, and in the original A3Lite that would have been enough to send the node to the Sleeping Node mode. However, in this protocol, that decision depends on knowing if the node was sensing-covered by any other node. In the case where the node was sensing-covered, the node will be sent to the Sleeping Node mode; otherwise, the node will change its state to Active Node.

Every node that reaches the Active Node state will send a Sensing Coverage Message to its neighbors in order to notify that it has been selected to be active. The message complexity of this protocol, compared to A3Lite, is at most $3 n$ in the case all nodes are selected to be active. Figure 5.2 shows the finite state machine of the A3CovLite algorithm.

\subsection{The $\alpha$-Coverage Sensing Coverage Definition}

After comparing the performance of the distributed A3-based algorithms for connectedcoverage, it was observed that while the algorithms covered almost $99 \%$ of the area in dense networks, they needed many more active nodes than the optimal deployments. In fact, the number of nodes was similar to that of the hexagon-based grid showed in [69, 


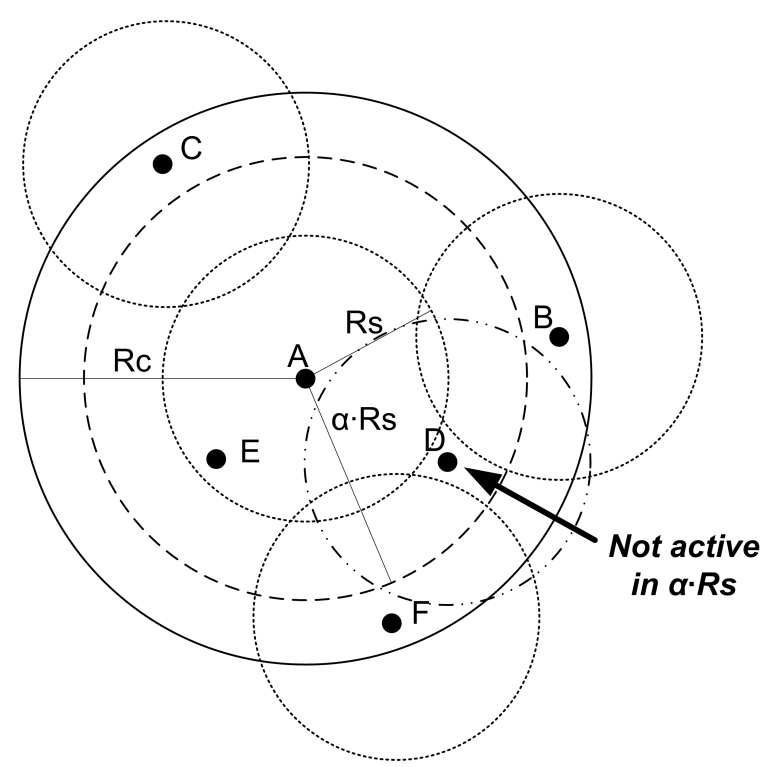

Figure 5.3: Example of $\alpha$-coverage.

72], which is the one that requieres the greatest number of active nodes among the optimal grids. After some insight into the way the distributed protocols worked, it was concluded that the selection criteria was mainly responsible. Remember that the nodes that are to be added to the active set, are those which are farther away from their parents and with the most remaining energy. The selection of these distant nodes creates small holes in the coverage that are filled by additional nodes, although these do not necessarely contribute much in terms of newly covered area.

Figure 5.3 shows an example of this situation. Nodes B, C, D and F would become active under the current definition of sensing coverage; however, it is easy to see that the contribution of node D to the covered area is minimal, because it is located right after the sensing radius of $\mathrm{A}$.

In other cases, the opposite can be happening. Some applications require a higher level of coverage than the one offered by the original selection policy. For example, the same Figure 5.3 shows the example of node $\mathrm{E}$ which is not selected because it has been sensing- 
covered by its parent node $\mathrm{A}$, but $\mathrm{E}$ is a node that could extend considerably the sensing coverage of the topology due to the fact that no other node is offering coverage near its position.

One solution is to "virtually" define the sensing coverage in order to increase or decrease the number of eligible nodes for coverage, depending on the requirements of the application. Under this new idea, a node is sensing-covered if $\forall x, y \in V, d(x, y) \leq \alpha R_{\text {Sense }}$, where $0<\alpha \leq R_{\text {Comm }} / R_{\text {Sense }}$. The main cases will be the ones of $\alpha \approx 0$, which will select most nodes to remain active; the case of $\alpha=1$ which will produce a similar result to the original version of sensing coverage, and the case of $\alpha=R_{\text {Comm }} / R_{\text {Sense }}$ which will produce a similar result to the case when the communication and sensing radii are equal. An example of the application of this new definition is also shown in Figure 5.3. If the new definition is used with $\alpha=1.3$, node $\mathrm{D}$ would not be included. The main source of savings in number of nodes is that most of the nodes in the belt between $R_{\text {Sense }}$ and $\alpha R_{\text {Sense }}$ will be filling up holes left by the selection of the nodes with higher priority, farther away from the parent node. If $\alpha=0.7$, then nodes $\mathrm{D}$ and $\mathrm{E}$ would be selected to be active, which will not represent any savings in energy, but will provide the best coverage ratio for the topology.

It is obvious that this new definition affects the total covered area by the topology, therefore the selection of the $\alpha$ parameter is very important. In the performance evaluation of the $\mathrm{A} 3 \mathrm{Cov}$ and $\mathrm{A} 3 \mathrm{CovLite}$ protocols, two different $\alpha$ values were tested in order to illustrate the impact of this parameter in the selection policy: $\alpha=1.3$ and $\alpha=0.7$. After performing experiments in a small sample of scenarios with different $\alpha$ values, an interesting trend was found: from $\alpha=R_{\text {Comm }} / R_{\text {Sense }}=1.7$ until $\alpha=1.3$, the growth of covered area versus the number of nodes followed a linear behavior; however, from $\alpha=1.3$ until $\alpha=1$, the number of nodes increased considerably faster than the area 
coverage. Even though this characteristic may not apply for all possible scenarios, it provides a good starting point for analysis. In the case of $\alpha=0.7$, the value was selected because it was at the same distance from the $\alpha=1$, but in the opposite direction, and just as a way of comparison.

In the performance evaluation section it will seen that the covered area for $\alpha=1.3$ falls between $80 \%$ and $90 \%$ in sparse networks and between $93 \%$ and $95 \%$ in dense networks, while reducing considerably the number of active nodes. In the other case, the covered area for $\alpha=0.7$ in which falls between $90 \%$ and $100 \%$ in sparse networks and between $99 \%$ and $100 \%$ in dense networks, having as a consequence a subtantial increase in the number of active nodes. More details on the performance using the modified sensing coverage will be presented in the next section.

\subsection{Performance Evaluation}

In this section, the complete A3 family of distributed protocols is compared with the theoretical bounds for connectivity and coverage, and two other distributed coverageoriented protocols, all of them presented in Section 2.3.3.

For the comparison with the theoretical bounds, three sets of experiments are included. The first set of experiments includes those experiments in which the communication and sensing radii are the same $\left(R_{\text {Comm }}=R_{\text {Sense }}\right)$. In this case, the A3 protocols are compared with the optimal deployments described in [69] and solutions to the packing problem found in the literature. This case also provides a scenario to compare connectivity-oriented and coverage-oriented protocols as equals.

The second set considers the case in which the communication radius is $\sqrt{3}$ times the sensing radius $\left(R_{\text {Comm }}=\sqrt{3} R_{\text {Sense }}\right)$. As explained before, this is the radius that guarantees 
connectivity and coverage with the minimum number of nodes. Here, the optimal deployments described in [72] are used for comparison. Finally, the third set of experiments considers the $\alpha$-coverage cases with $\alpha=1.3$ and $\alpha=0.7$, using a communication range of $R_{\text {Comm }}=\sqrt{3} R_{\text {Sense }}$.

Each of the experiments described before were performed in sparse and dense networks.

In all the experiments presented in this section, the concepts of sparse and dense networks is not only related to the node density in the area, but to the level of connectivity, which is more related to the node degree networks. In other words, in sparse networks each node has a smaller neighborhood than in the dense networks. The definition of these parameters is based on the Critical Transmission Range (CTR) concept, i.e. the minimal communication range needed to provide a connected topology in dense networks. In this experiment, the CTR formula from Penrose and Santi, defined in Equation 2.1, was utilized.

The evaluation under sparse and dense networks is very important because most of the centralized optimal solutions for connected-coverage presented in Section 2.3.3 assume very dense network scenarios. As it will be shown later, this is not a restriction for the A3 family of distributed protocols, which also perform very well in sparse scenarios.

In all scenarios, the number of active nodes and the percentage of covered area given connectivity are utilized as the main performance metrics for comparison. The complete set of experiments with the A3 distributed algorithms were performed with Atarraya.

\subsubsection{Comparison with Theoretical Deployments}

The process to plot and compare the results for the packing problem, the optimal deployments and those obtained from A3 and A3Lite simulation results, is performed as follows. 
Table 5.1: Simulation parameters for coverage-oriented protocols.

\begin{tabular}{|l|l|}
\hline Parameter & Value \\
\hline Deployment area & $600 \mathrm{mx} 600 \mathrm{~m}$ \\
\hline Node distribution & Uniformly distributed within the area \\
\hline Instances per topology & 50 instances \\
\hline A3 Weights & $W_{E}=0.5, W_{D}=0.5$ \\
\hline Energy consumption & $E_{\text {elec }}=50 \mathrm{~nJ} /$ bit $; E_{\text {amp }}=10 \mathrm{pJ} /$ bit $/ \mathrm{m}^{2}$ \\
\hline Packet sizes & Short Msg $=25$ Bytes $;$ Long Msg=100Bytes \\
\hline A3 Timers & $t_{0}=1.5, t_{1}=30, t_{2}=15, t_{3}=60$ \\
\hline Energy Parameters & as described in Table 3.1 \\
\hline
\end{tabular}

In all cases, the area of deployment was established to be $600 m \times 600 m$, and the sensing and communication radii have the value of $R_{\text {Sense }}, R_{\text {Comm }}=R_{\text {Sense }}$. For the packing problem, given these two values, the plotted result was the maximum number of nonoverlapping circles of radius $R_{\text {Sense }}$ that can be fitted in the area, which was determined based on existing solutions in the literature [76]. It is worth mentioning that there is no "formula" or equation to solve this problem. The solution to the packing problem number is the lower bound of connectivity and coverage for that particular scenario. Then, based on the number of circles, the addition of their individual areas over the total area is sensing coverage ratio, as shown in Equation 5.1.

$$
\text { Coverage }_{\text {Packing }}=\frac{n \cdot R_{\text {Sense }}^{2} \cdot \pi}{l^{2}}
$$

where $n$ is the packing number, and $l$ is the side of the square.

The figures for all the results in this section are structured in this manner: the x-axis of the results represents the sensing radius $R_{\text {Sense }}$ as a ratio of the side of the area of deployment. So, for example, a value of 0.5 means that the sensing radius is half the size of the side of the deployment square, or $300 \mathrm{~m}$ in our case. In the y-axis, the number of active nodes 
and the covered area will be shown in their respective plot. Table 5.1 shows the main parameters utilized in the simulation experiments.

For the optimal deployments described in [69], it is always considered that they achieve $100 \%$ coverage. The number of nodes produced by each deployment grid was obtained from the inverse of the density Equations 2.6 and 2.7 with the radius as a ratio of the area side, as shown in Equation 5.2, and $\Theta=\pi / 2$ and $R_{\text {Comm }} / R_{\text {Sense }}=\sqrt{3}$ to calculate $d_{R H O}$.

$$
n_{\text {deployment }}=\frac{1}{F_{\text {density }}\left(\frac{R_{\text {Sense }}}{l}\right)}
$$

where $F_{\text {density }}$ is the density function of the given deployment grid, and $\frac{R_{\text {Sense }}}{l}$ is the ratio of the sensing radius versus the side of the square. This last operation is necessary because the density functions were designed to work with a unit square.

Finally, for the A3 algorithms, the first parameter needed was the initial size of the topology. The two optimal deployment techniques do not need an initial topology given that they provide an optimal one from the beginning. In the case of the distributed algorithms, an initial topology is necessary in order to have spread enough nodes to have most of the deployment area covered, and enough density to guarantee connectivity.

As it has been discussed earlier, Equation 2.1 provides the Critical Transmission Range (CTR) for a scenario where the number of nodes and the area side of the deployment area are known. Given that in these experiments just the area of deployment and the desired sensing radius are known, the size of the topology is calculated by testing different values for the total number of nodes parameter, until the CTR formula provides radii that are equal to 1 and 0.5 times the desired communication radius, in order to provide $1 C T R$ and $2 C T R$ (sparse and dense) networks, respectively. The process was repeated for all the sensing radii considered the experiments, in order to assess the performance of the 
Table 5.2: Radii and topology sizes for coverage-oriented protocols.

\begin{tabular}{|c|c|c|c|}
\hline$R_{\text {Sense }}$ & $R_{\text {Comm }}$ & $N_{\text {Dense }}$ & $N_{\text {Sparse }}$ \\
\hline 300 & 520 & 21 & 5 \\
\hline 180 & 312 & 83 & 12 \\
\hline 150 & 260 & 129 & 21 \\
\hline 120 & 208 & 225 & 39 \\
\hline 105 & 182 & 311 & 56 \\
\hline 90 & 156 & 445 & 83 \\
\hline 75 & 130 & 683 & 131 \\
\hline 60 & 104 & 1100 & 225 \\
\hline 51 & 88 & 1650 & 332 \\
\hline 45 & 78 & 2200 & 446 \\
\hline 39 & 68 & 3040 & 623 \\
\hline 34 & 59 & 4000 & 855 \\
\hline
\end{tabular}

algorithms with different sensing ranges. The selection of the different radii was based on the available results for the circle packing problem in [76].

Let it be said that all the random topologies used for this experiment were initially connected. The covered area was calculated using a graphic method: assuming each pixel as a square of $1 m \times 1 m$, the covered area can be obtained by counting all pixels covered by the active nodes and then dividing that amount by the total amount of pixels on the graphical representation of the topology. Table 5.2 shows the complete list of parameters $R_{\text {Sense }}, R_{\text {Comm }}=\sqrt{3} R_{\text {Sense }}, n_{\text {Sparse }}$ and $n_{\text {Dense }}$. The values for $R_{\text {Comm }}$ are only used in the second and third sets of experiments.

\subsubsection{Experiment 1: Same Radii}

In these experiments it is assumed that $R_{\text {Comm }}=R_{\text {Sense }}$. This assumption is made by [69] and creates a scenario in which connectivity and coverage become the same problem. Figures 5.4 and 5.5 show the results of the experiments. Several general observations can be made from the figures. For example, the performance of the optimal deployments 
completely agree with the theoretical expectations. The solution to the packing problem always provides the lower bound in number of nodes and coverage, regardless of the network density. The strip deployment is the next best, right after the packing, followed by the hexagon and the square in that order. The optimal deployments always provide $100 \%$ coverage; they are designed to do so. The only difference is that, as expected, the number of active nodes increases inversely with the sensing radius. With regard to the family of A3 protocols, they also behave as expected: the pairs of Non-Lite and Lite protocols show similar behaviors in terms of number of nodes inside their own categories. This is explained by the fact that the respective pairs of protocols share the same inner mechanisms for selection except for the sensing covering issue, but given that in this case the communication and sensing radii are the same, the connectivity and the coverage turn into the same problem: a node covered by the communication range will be also covered by the sensing range.

In terms of the number of nodes, in sparse networks all four A3 algorithms present similar performance, which is expected given that there are not too many choices to grow different trees. In this case, they perform similarly to the hexagon, between the square and the strip deployments. With the smallest radius of 0.05 , equivalent to 34 meters and of 855 nodes in the sparse deployment, the A3 algorithms utilized around 250 nodes, which is around $30 \%$ of the total number of nodes. In dense networks it can be seen how the Lite versions need fewer active nodes than their Non-Lite counterparts. This difference is explained by the methodology of growing the tree of the algorithms: the Lite versions give more time for new branches to grow, which tends to reduce the possibility of leaving unvisited nodes for other waiting candidate nodes. However, in this case, the performance of the algorithms compared to the optimal deployments is worse. The Non-Lite algorithms perform the worst of all, above the square, and the Lite versions just below the square. 


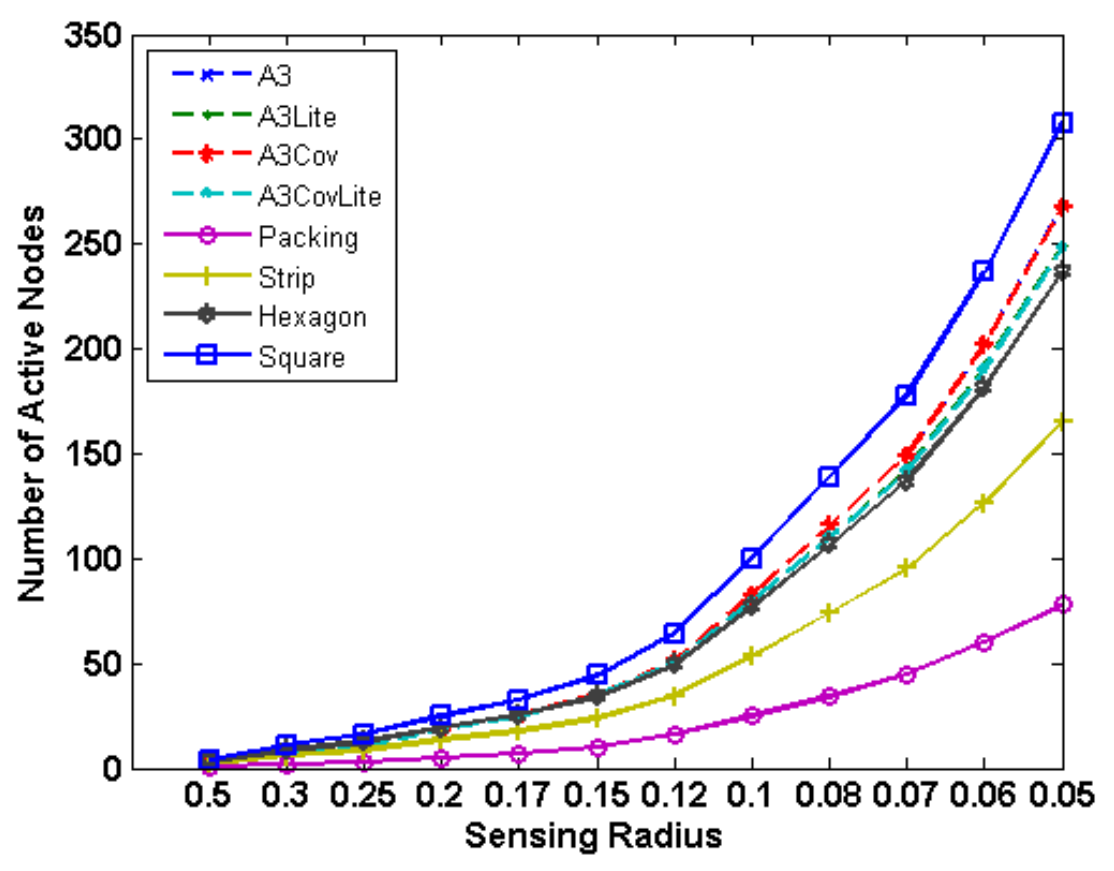

(a) Number of active nodes in sparse networks.

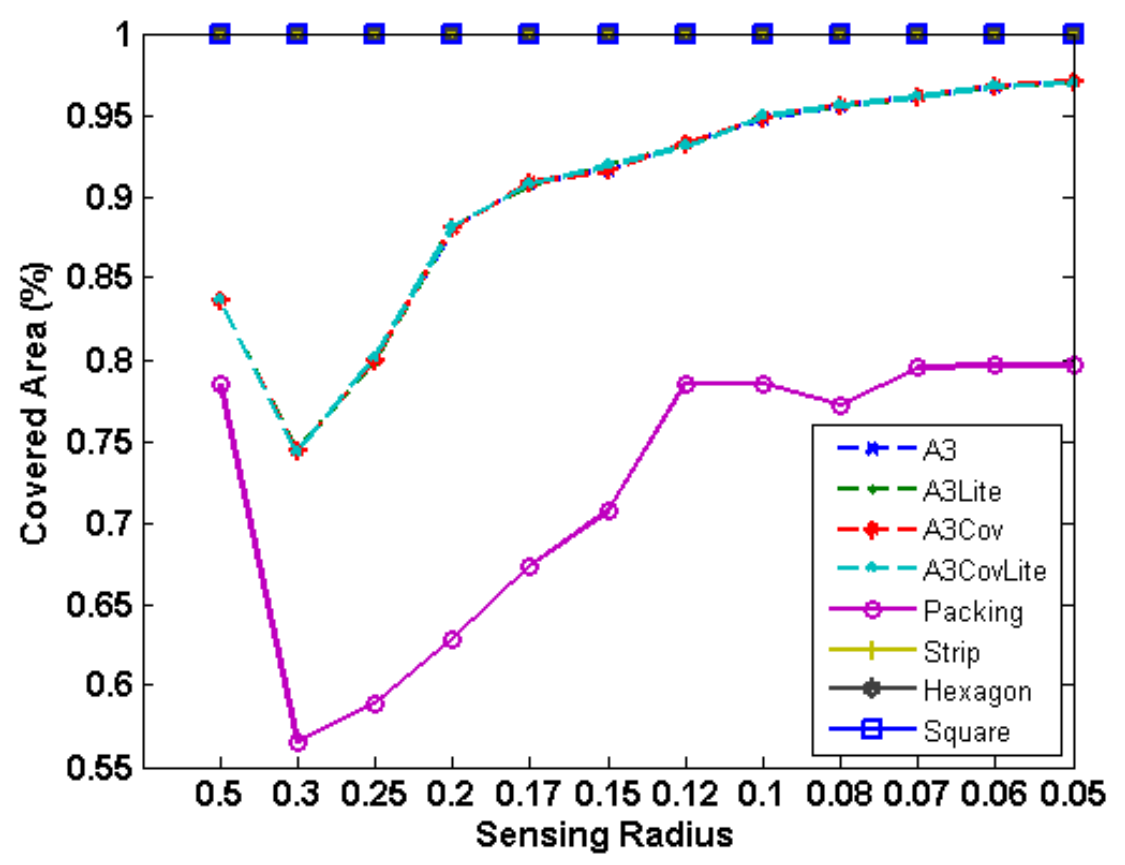

(b) Connectivity-coverage in sparse networks.

Figure 5.4: Performance in sparse networks when $R_{\text {Comm }}=R_{\text {Sense }}$. 


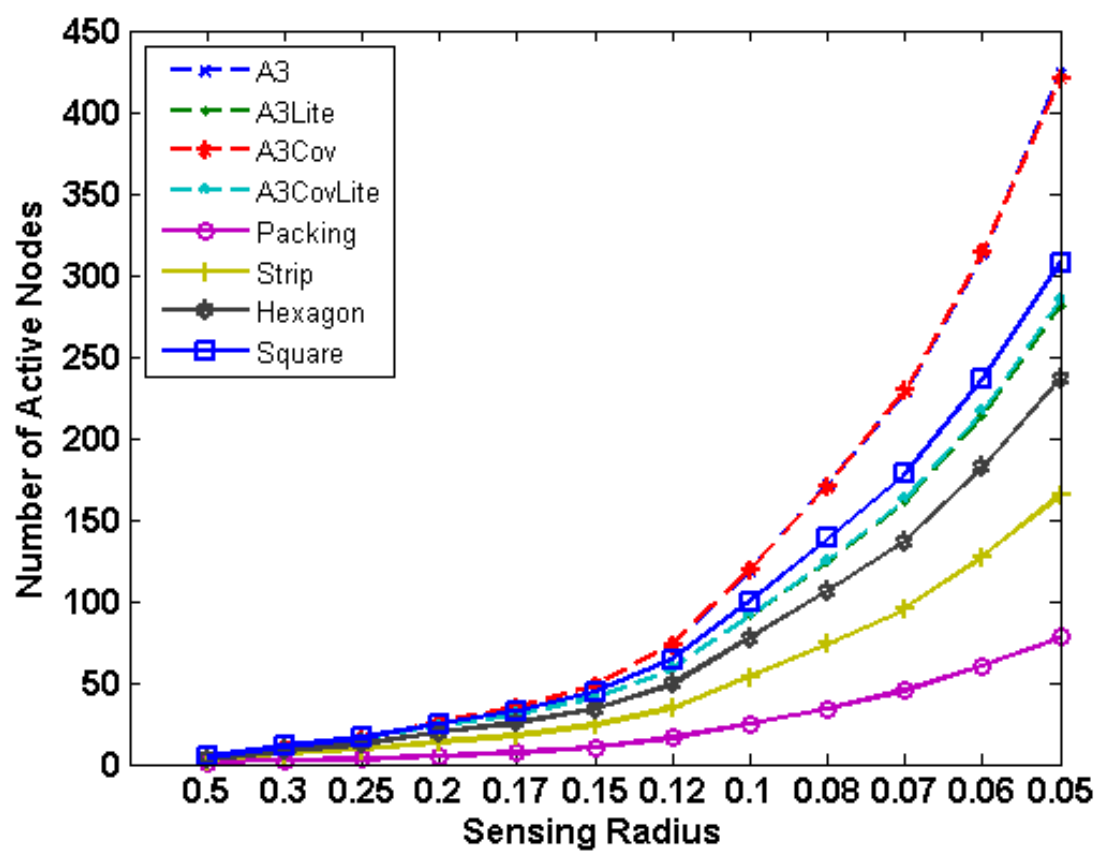

(a) Number of active nodes in dense networks.

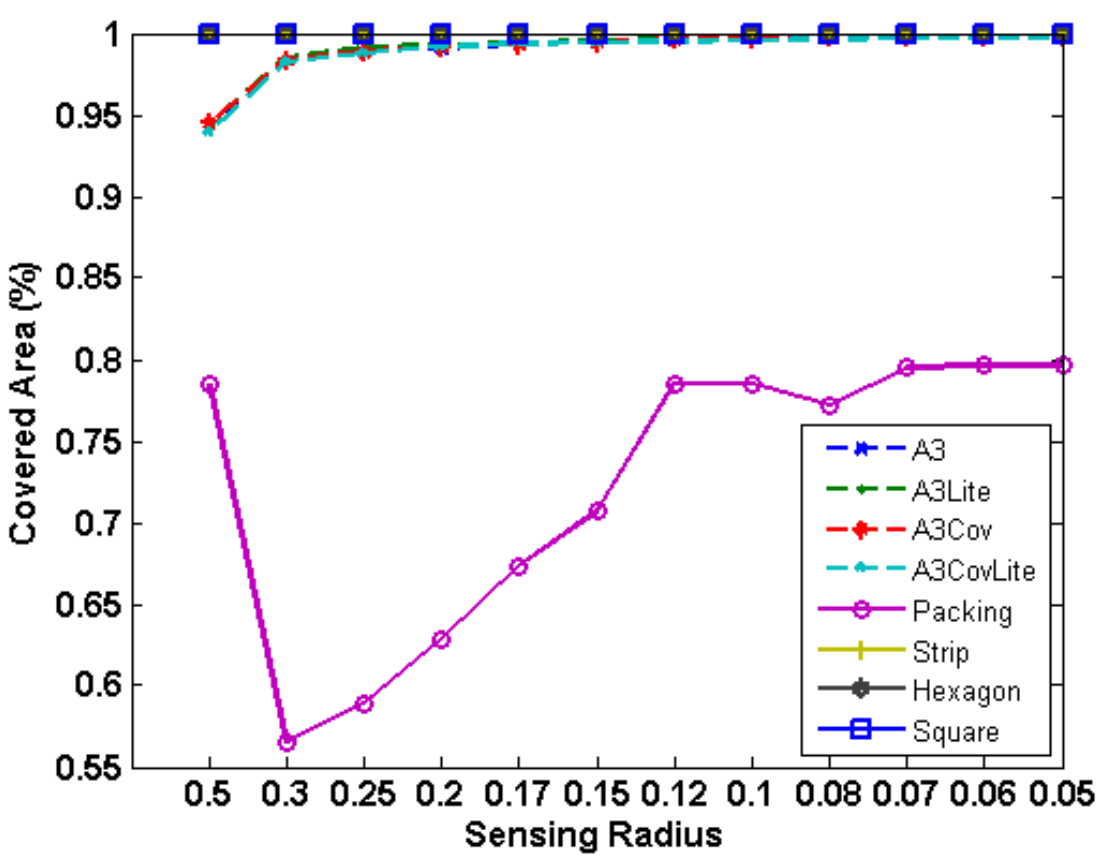

(b) Connectivity-coverage in dense networks.

Figure 5.5: Performance in dense networks when $R_{\text {Comm }}=R_{\text {Sense }}$. 
Nonetheless, even in the worst case, the distributed protocols utilize around $10 \%$ of the nodes to build the reduced topology, which is a considerable reduction from the sparse networks. It is worth mentioning that the performance of the optimal deployments shown in the figures is theoretical, as it assumes that the algorithms find nodes in the precise locations needed by the geometry in question. Of course, this assumption will not hold true in all cases, even less so in sparse networks. Therefore, in a real implementation, it is expected that the performance of a distributed algorithm implementing these optimal deployments will be worse, which in turn makes the A3 algorithms look good better. In terms of coverage, in sparse networks the A3 algorithms improve the coverage considerably compared with the lower bound of the packing problem but they are still away from the $100 \%$ coverage provided by the optimal deployments, especially when large sensing radii are used. However, the A3 algorithms achieve a coverage of $90 \%$ or better for sensing radii equal or smaller than 0.17 . It is worth noticing that the simulation results presented in [69] also assumed a target $90 \%$ coverage because otherwise the number of active nodes to provide $100 \%$ coverage increased considerably.

The behavior experienced by the packing problem and the $\mathrm{A} 3$ algorithms around a radius of 0.3 is due to different reasons: in the case of the packing problem, a radius of 0.3 is very inconvenient because it only allows placing 2 circles inside the square, leaving a great amount of uncovered space. As the radius gets smaller, more circles can be packed tighter, decreasing the amount of uncovered space. In the case of A3, that behavior is more related to the low density of nodes in sparse topologies: a small number of connected nodes usually makes it likely that they are not too spread out within the area, implying that they are contained in a small convex hull compared to the total deployment area. Remember that A3 does not need to cover more area than the one necessary to cover the nodes in the network, so in average, the results show that a covered area of $75 \%$ is 
enough to cover all the 12 nodes with radius of 0.3 . In the case of dense networks, as shown in Figure 5.5(b), the covered area is near to $99 \%$ in most cases because in this case the nodes are more likely to be spread throughout the entire area, thus the convex hull is more equivalent to the deployment area, and covering the nodes would be almost the same as covering the deployment area. These results also confirm the statement from [71] in which the coverage of node positions is assumed as an approximation of the coverage of the entire area.

In summary, when $R_{\text {Comm }}=R_{\text {Sense }}$, the A3Lite distributed algorithm is the best choice, because A3Lite needs the least number of active nodes, while providing the same coverage and using the least number of messages among all the A3 protocols.

\subsubsection{Experiment 2: Different Radii}

As stated before, it is assumed in these experiments that $R_{\text {Comm }}=\sqrt{3} R_{\text {Sense }}$ and the parameters in Table 4.1 are used. Similarly to the last section, the solution to the packing problem is the lower bound for number of active nodes and coverage. Even though the optimal distributions from Experiments 1 and 2 share some geometries, they are ranked differently in terms of number of active nodes: the rhombus is the closest distribution to the packing, followed by the strip, the square and the hexagon, in that order, regardless of network density. The hexagon performs worse than the square because of the double line of nodes needed to provide connectivity.

As expected, the Cov versions need more active nodes in both scenarios, being close to the square in sparse networks, and to the hexagon in dense networks. On the other hand, the Non-Cov versions not only need fewer nodes but perform very close to the strip and the rhombus in sparse and dense networks respectively, which are the best performers in 


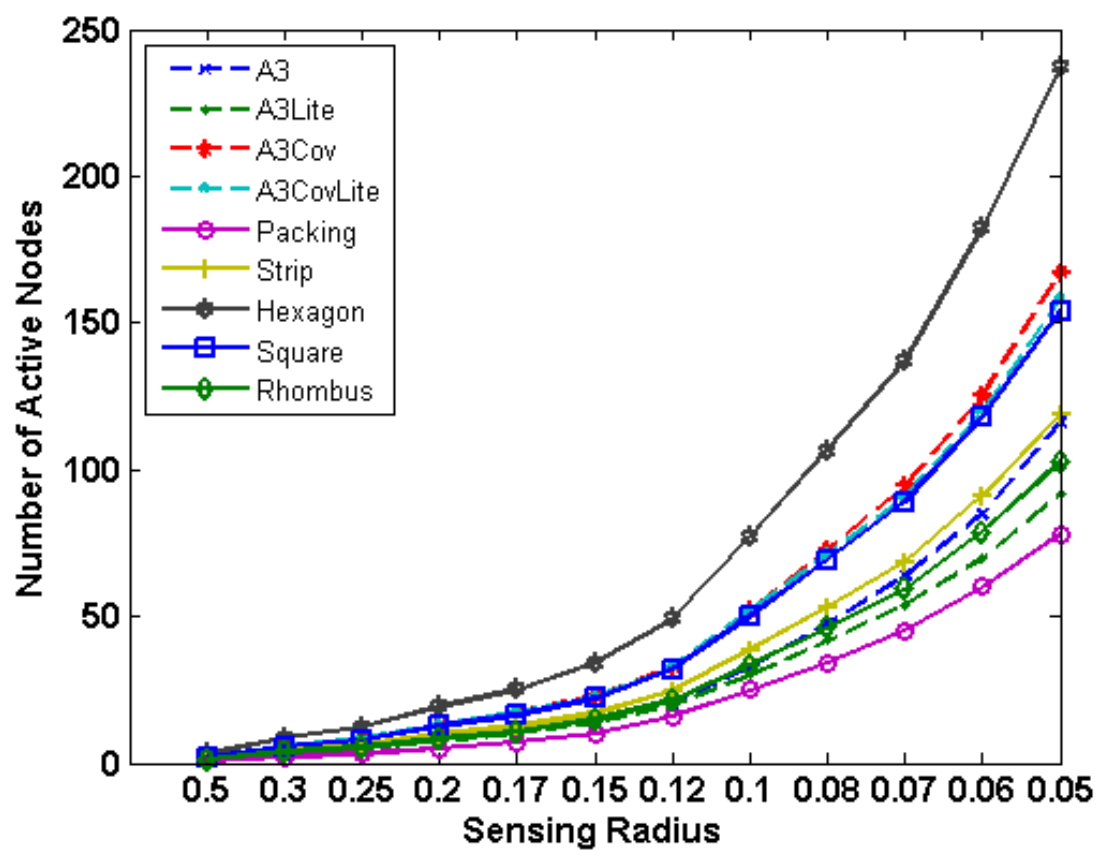

(a) Number of active nodes in sparse networks.

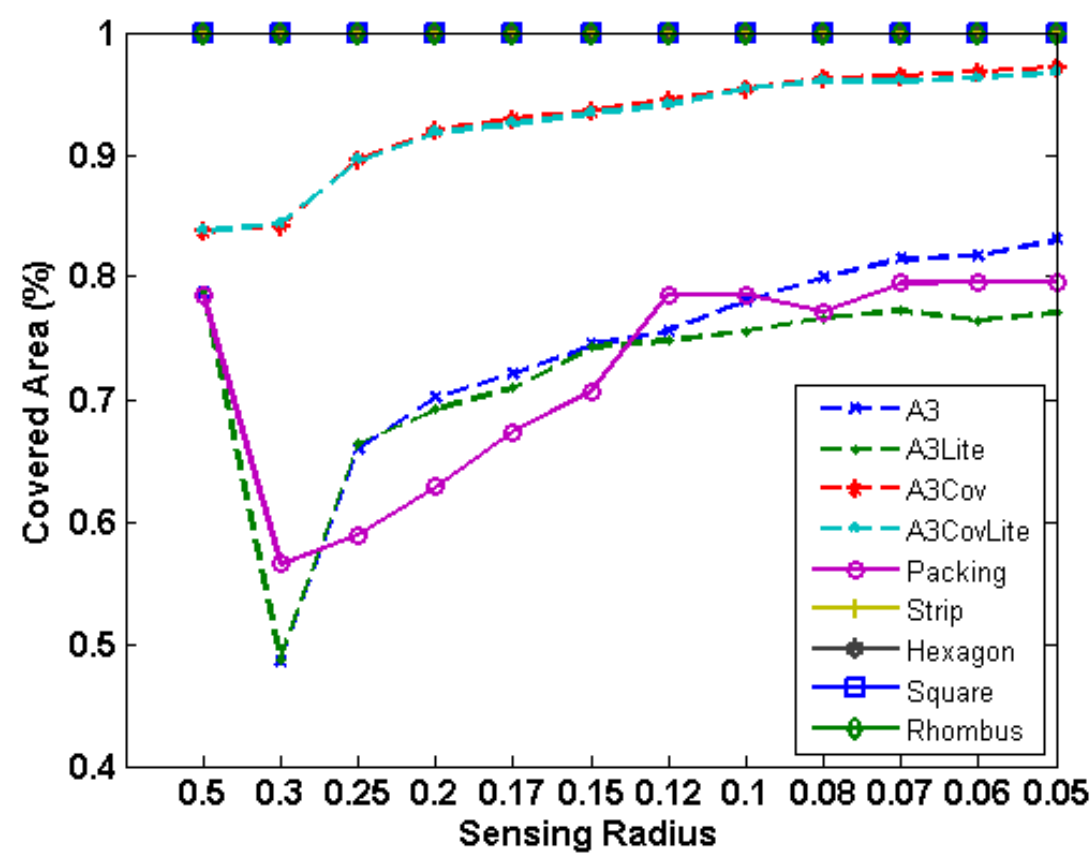

(b) Connectivity-coverage in sparse networks.

Figure 5.6: Performance in sparse networks when $R_{\text {Comm }}>R_{\text {Sense }}$. 


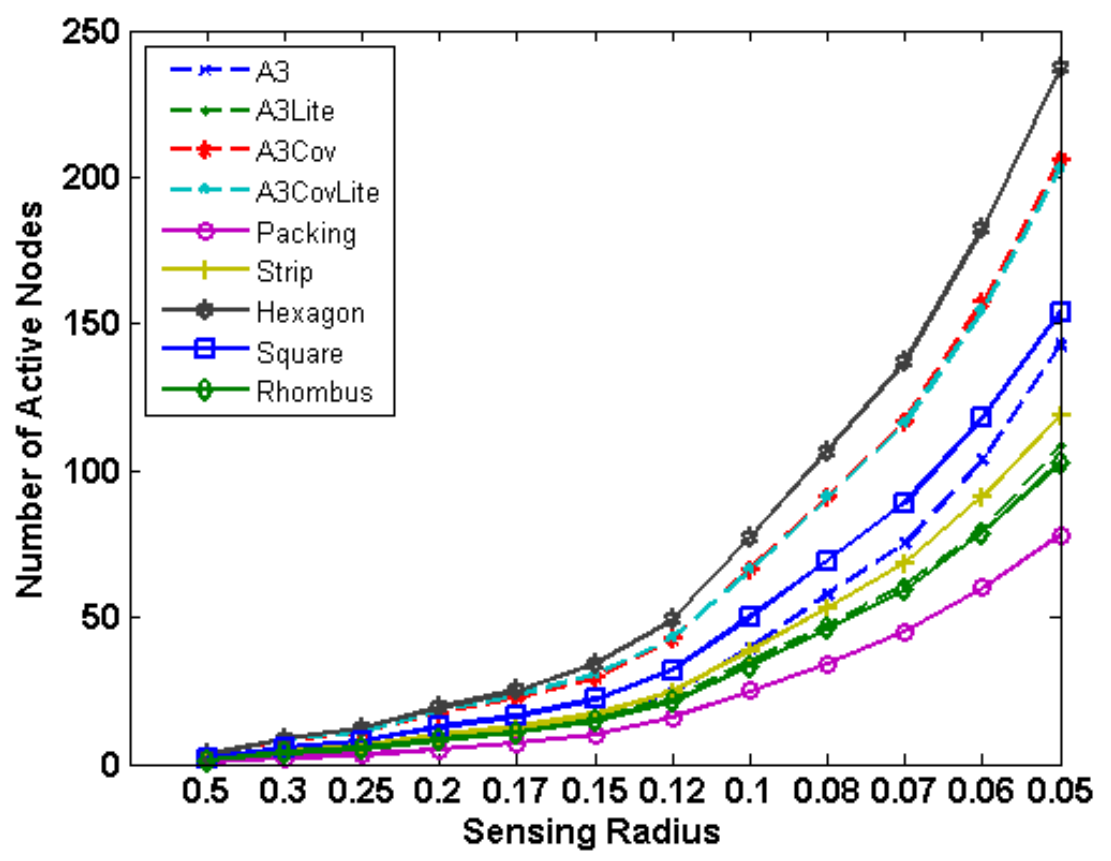

(a) Number of active nodes in dense networks.

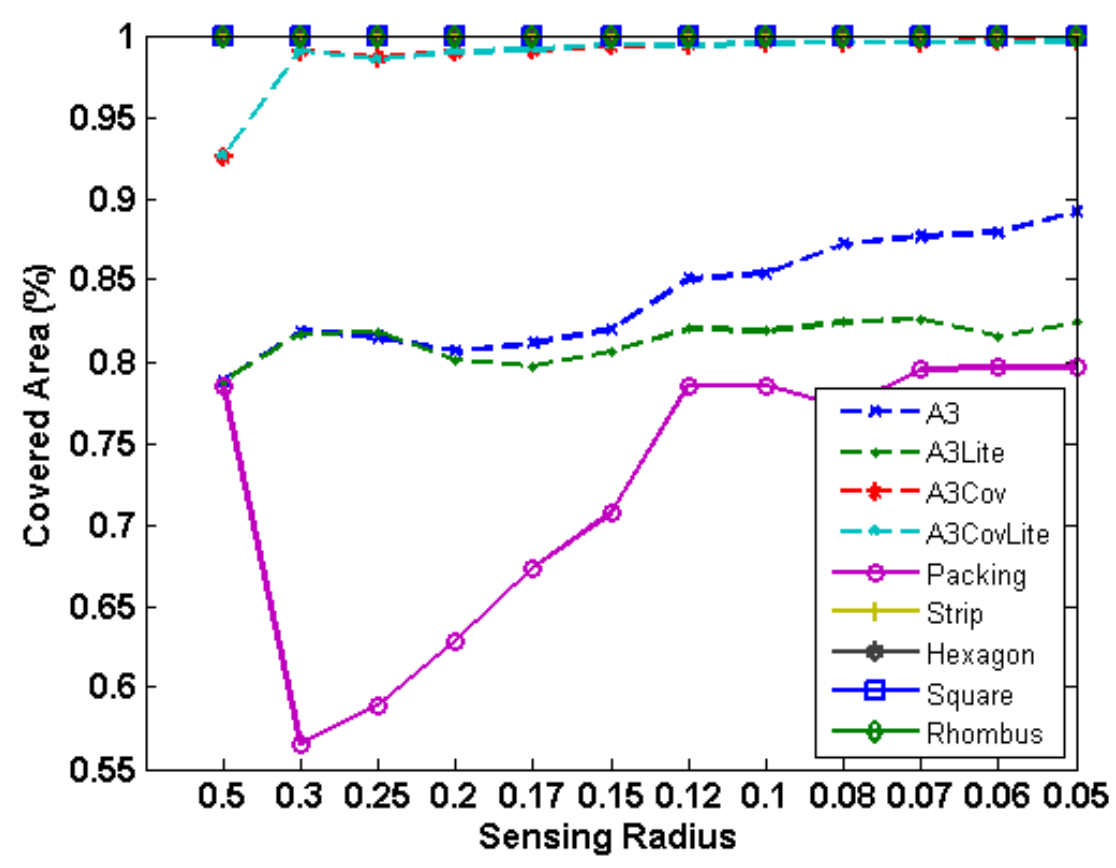

(b) Connectivity-coverage in dense networks.

Figure 5.7: Performance in dense networks when $R_{\text {Comm }}>R_{\text {Sense }}$. 
the optimal deployments. This is explained by the fact that the Non-Cov protocols do not include any extra nodes for coverage, because connectivity is their only goal.

Another fact to be analyzed is that, in general, the number of nodes required to offer connectivity in Experiment 2 is less than in Experiment 1. This can be explained by the fact that with a larger $R_{\text {Comm }}$, the protocols can build a more spread out topology with less overlapping of sensing areas and therefore, a connected topology with fewer nodes. For example, using a radius of 0.05 , A $3 \mathrm{Cov}$ needs around 425 active nodes in the case that $R_{\text {Comm }}=R_{\text {Sense }}$ and only 200 in this scenario with $R_{\text {Comm }}=\sqrt{3} R_{\text {Sense }}$. The disadvantage is that since the communications radius is larger than the sensing radius, the protocols connect the network with very few nodes but leave many spaces uncovered. This is in fact shown in Figures 5.6(b) and 5.7(b), where the poor coverage performance of the A3 algorithms can be seen.

In the case of area coverage, the results match the expectations. Now the Cov versions perform considerably better than the Non-Cov versions, as the Cov algorithms wake up many more nodes that were within the communication range but not within the sensing range of their parents. Of course, this is done at the expense of having more active nodes, which is also shown in Figures 5.6(a) and 5.7(a). The Cov versions provide the best coverage in both, sparse and dense scenarios with a coverage between $85 \%$ and $97 \%$, and between $92 \%$ and $99 \%$, respectively, but with a performance above $90 \%$ for a radius of 0.25 or smaller in all cases. However, in dense networks, these algorithms perform very close to the optimal deployment in most cases. The behavior around the 0.3 radius seen in Figure 5.4(b) is also observed here but in the sparse network scenario (Figure 5.6(b)) and for the Non-Cov versions only. Given that the communication range is $\sqrt{3}$ times larger than the sensing range, even fewer nodes are required to keep the network connected 
(somewhere around two and three nodes) compared to the results of Experiment 1, which required around 5 nodes. It is not possible for 2 or 3 nodes cover the whole area.

In summary, in the case of systems where $R_{\text {Comm }} \neq R_{\text {Sense }}$, there is no clear winner, and rather, trade offs exist. If coverage is not as critical as energy (network lifetime), the NonCov algorithms need fewer nodes to build the reduced topology. In this case, it would be better to use A3Lite, as it needs fewer number of nodes and messages. If coverage is very important for the application, then the Cov algorithms should be used. In this case, the A3CovLite is the best option mostly because of its lower message complexity.

\subsubsection{Experiment 3: Different $\alpha$-coverage}

These experiments are performed to show how the $\alpha$-coverage parameter introduced in Section 5.3 modifies the behavior of the A3Cov and A3CovLite protocols. The trade off is clear.

When the $\alpha$-coverage parameter assume values greater than 1.0, the $C o v$ versions perform a little bit worse in terms of coverage but a little bit better in term of the number of nodes compared with the results shown in Experiment 2. Taking A3CovLite with a radius of 0.05 as an example, in the case of sparse networks, Figures 5.8(a) and 5.8(b) show that it covers around $90 \%$ of the area with close to 100 nodes while Figures 5.6(a) and 5.6(b) show that the same algorithm without the $\alpha$-coverage parameter covers $97 \%$ of the area but utilizes close to 150 active nodes. In the case of dense networks, the numbers are $93 \%$ and 130 nodes versus $100 \%$ and 200 nodes.

As seen in the results, the difference between $\mathrm{A} 3 \mathrm{Cov}$ and $\mathrm{A} 3 \mathrm{CovLite}$ is now more noticeable in both number of active nodes and coverage. In the sparse scenario, the number of active nodes of the coverage protocols is located between the strip and the rhombus, with 


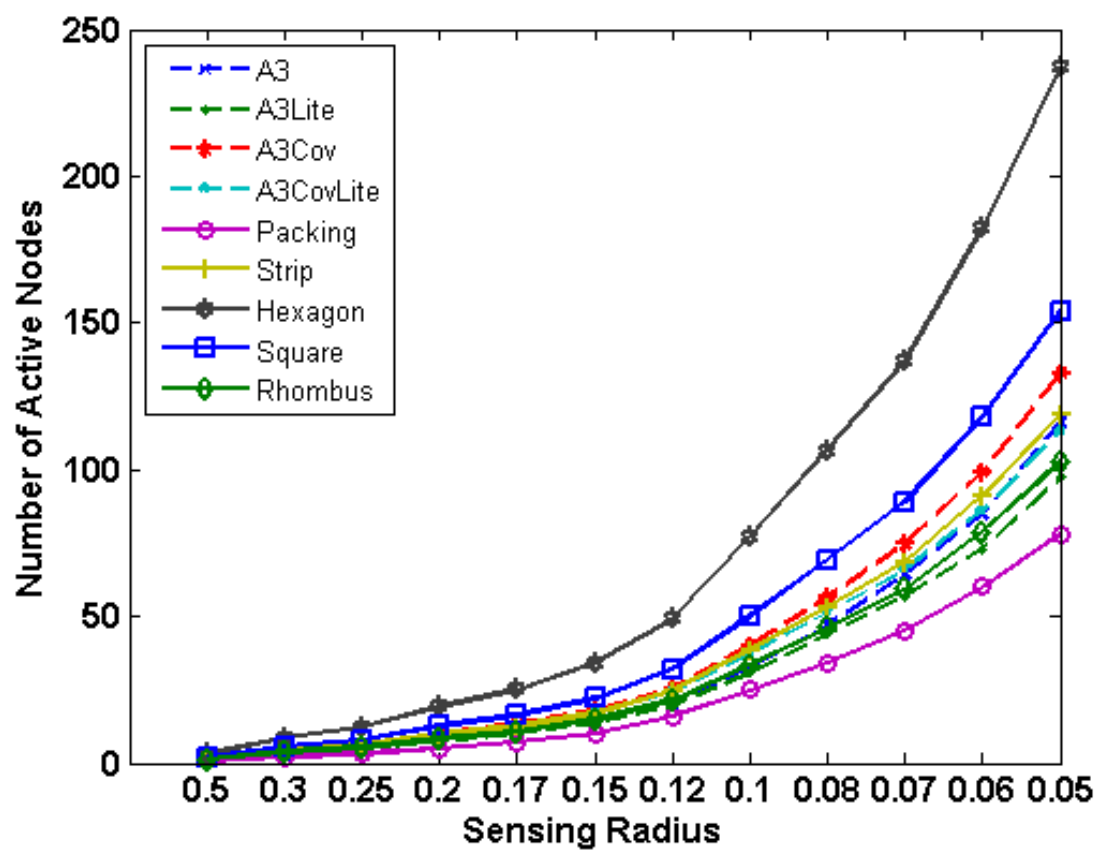

(a) Number of active nodes in sparse networks.

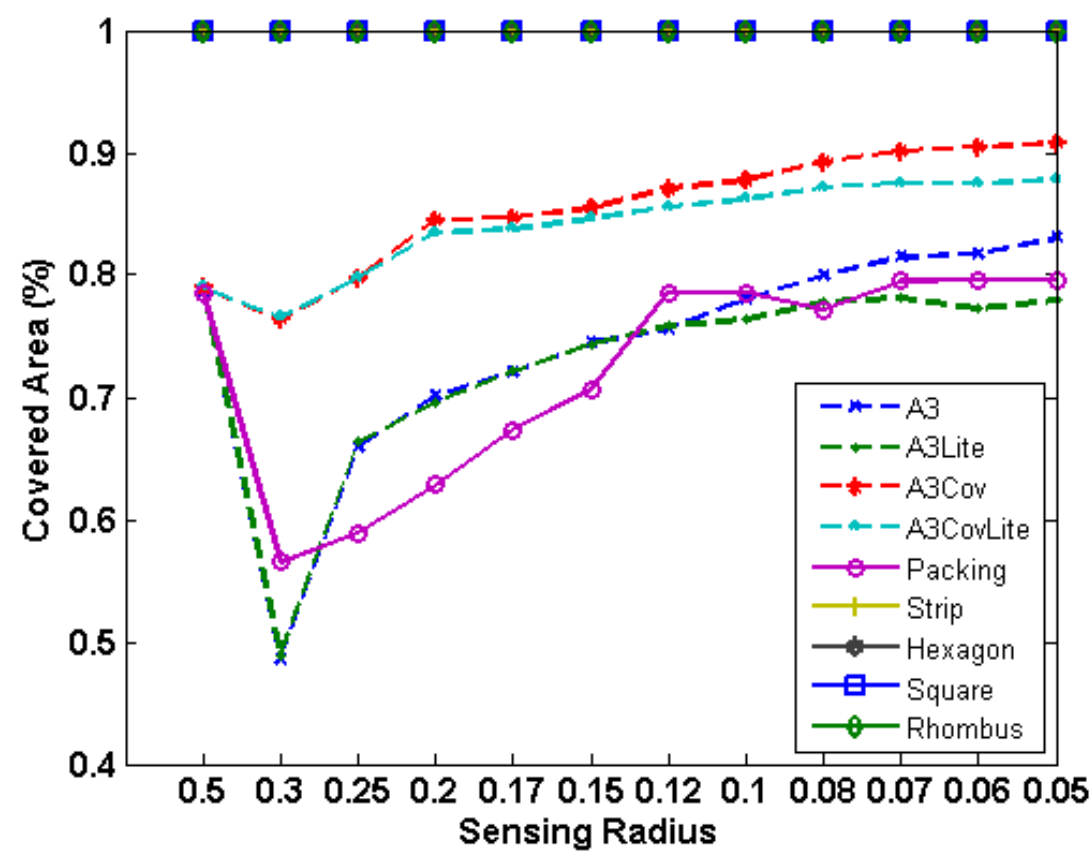

(b) Connectivity-coverage in sparse networks.

Figure 5.8: Performance in sparse networks when $R_{\text {Comm }}>R_{\text {Sense }}$ and $\alpha>1$. 


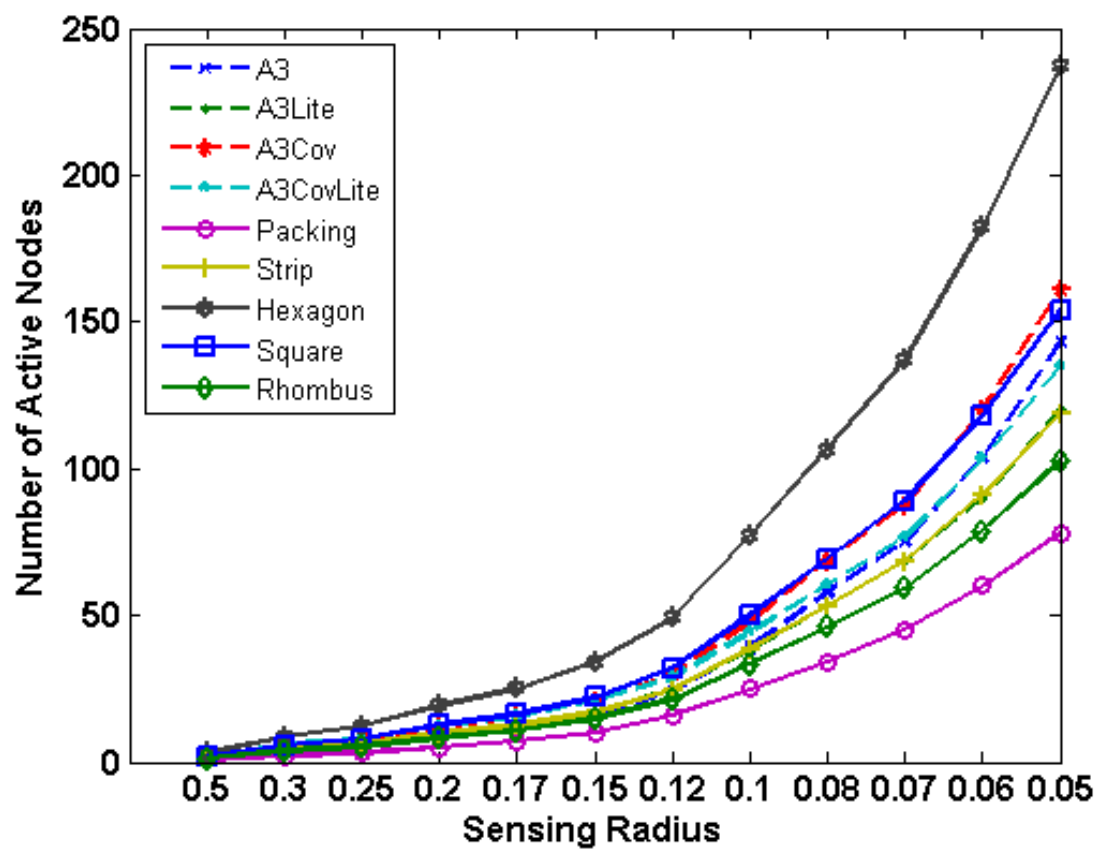

(a) Number of active nodes in dense networks.

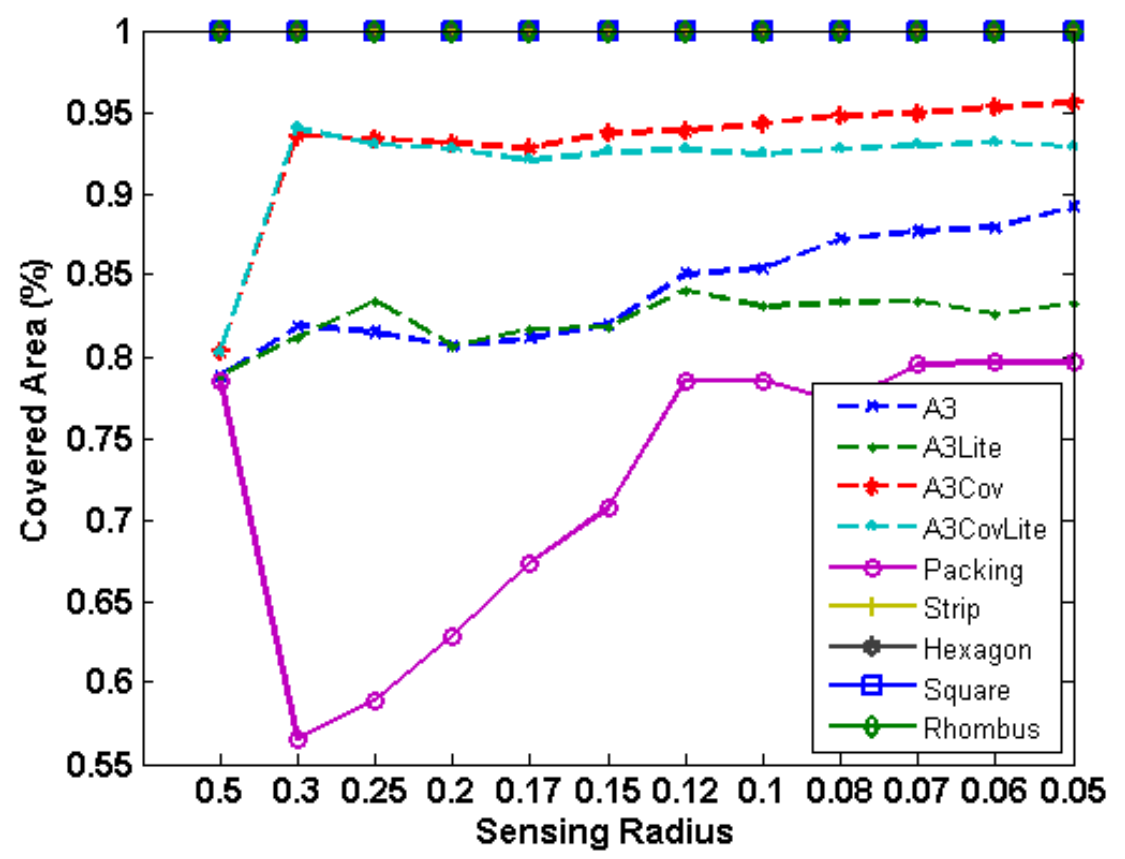

(b) Connectivity-coverage in dense networks.

Figure 5.9: Performance in dense networks when $R_{\text {Comm }}>R_{\text {Sense }}$ and $\alpha>1$. 
A3CovLite the closest to the minimum. As expected, these numbers are smaller than the ones in Experiment 2 because the virtual coverage of the $\alpha$ factor restricts the number of candidates to be selected for coverage purposes. In dense networks, the number of nodes of the $\mathrm{A} 3 \mathrm{Cov}$ protocol is similar to the square; in the case of the A3CovLite and the A3 protocols, the number of nodes is between the strip and the square, and the A3Lite protocol shows a behavior comparable to the strip distribution. The coverage suffered a decrease between 5\% and 7\% compared to the results on Experiment 2. In the other case, when the $\alpha$-coverage parameter assumes values less than 1.0 , the Cov versions offer an extended area of coverage compared to the other two versions; however, this increase comes with a great cost in the number of active nodes. In Figure 5.10(b) it can be seen how the protocols offer a area of coverage no less than $90 \%$ even with a sensing radius of 0.5 in the sparse networks. This level of coverage is only reached by the Cov protocols with $\alpha$-coverage parameter of 1.0 and 1.3 when the sensing radius is around 0.15 and 0.05 respectively, as seen in Figure 5.12(b), which represents a gain of almost $5 \%$ more coverage.

In the case of the dense networks, in Figure 5.11(b) it can be seen how the $\alpha$-coverage parameter keeps the coverage ratio at values higher than $99 \%$ for all sensing radii, which competes directly with the level of coverage provided by the optimal solutions. However, as in Figure 5.13(b), it can be see how the gain in coverage is at $8 \%$ for the first radius and not more than $2 \%$ in the rest of the scenarios compared to the values of the other $\alpha$ coverage parameters.

Now, as it can be seen in Figures 5.12(a) and 5.13(a) keeping that 90\% coverage in the sparse networks and the $99 \%$ coverage in the dense network, imposes a heave tax to the network: roughly double the number of active nodes are needed, compared to the ones required when the $\alpha$-coverage parameter is 1.0, and almost triple the nodes when the $\alpha$ - 


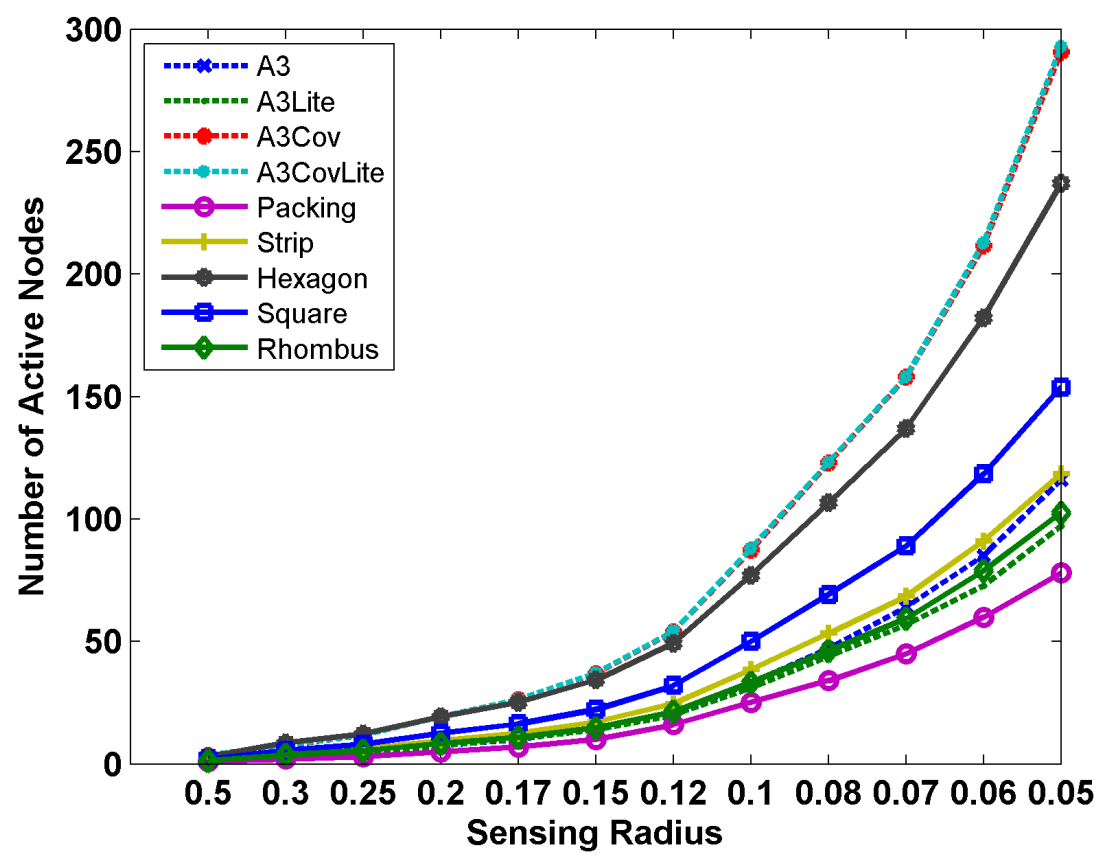

(a) Number of active nodes in sparse networks.

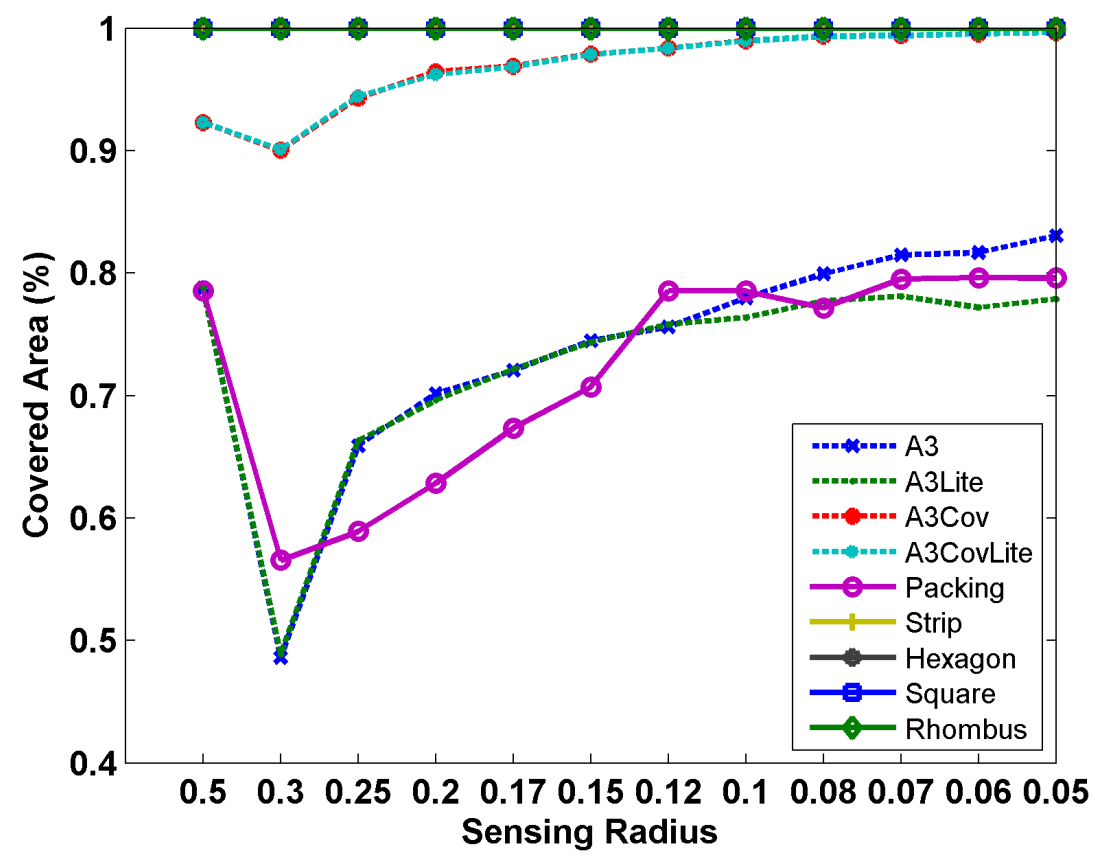

(b) Connectivity-coverage in sparse networks.

Figure 5.10: Performance in sparse networks when $R_{\text {Comm }}>R_{\text {Sense }}$ and $\alpha<1$. 


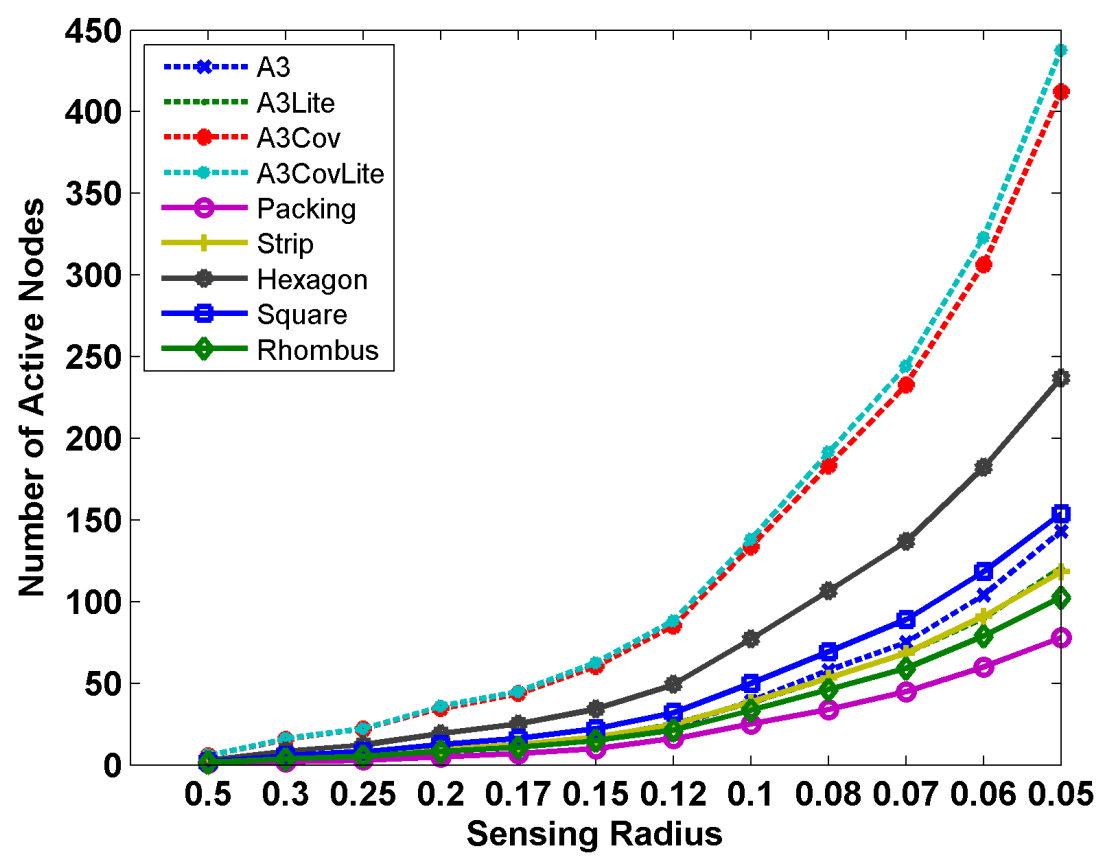

(a) Number of active nodes in dense networks.

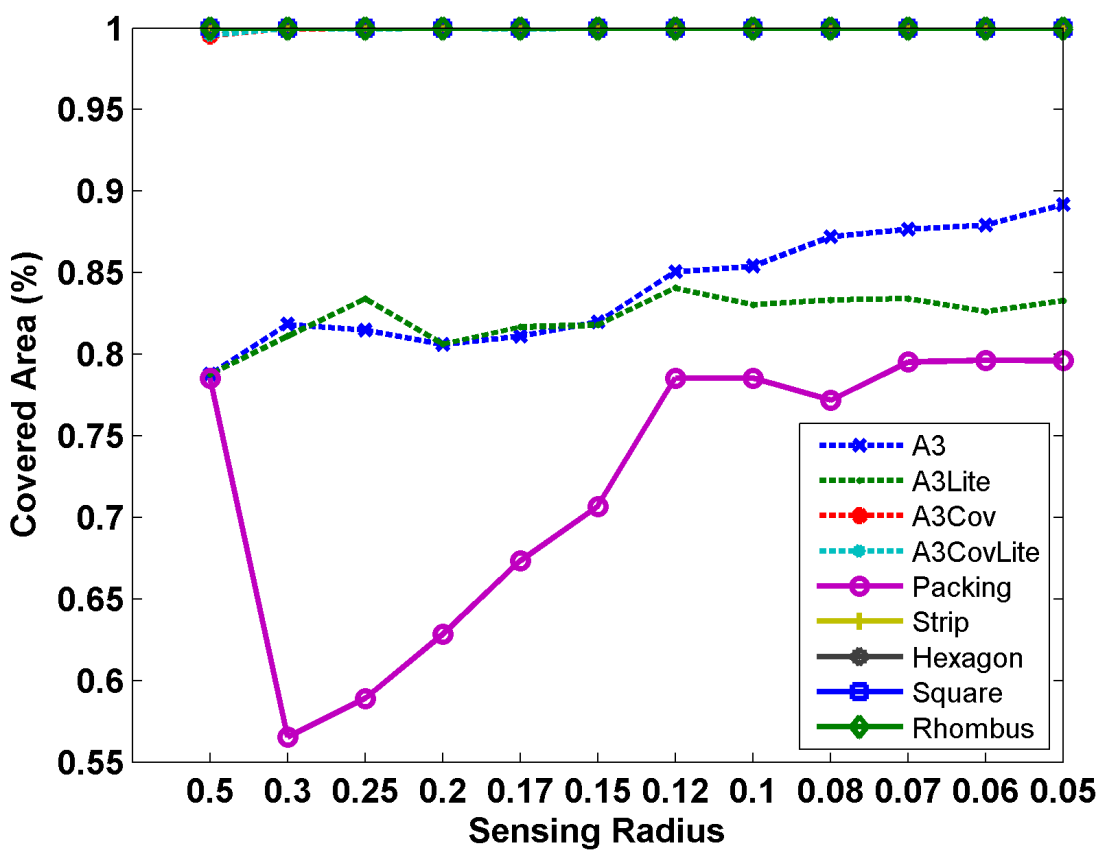

(b) Connectivity-coverage in dense networks.

Figure 5.11: Performance in dense networks when $R_{\text {Comm }}>R_{\text {Sense }}$ and $\alpha<1$. 
coverage parameter is 1.3. This increase removes the Cov protocols away from being the wort of the optimal solutions, as shown in Figures 5.10(a) and 5.11(a). This tradeoff may be excesive for most applications, but not for those in which the critical factor is coverage and not energy or lifetime.

In conclusion, it can be seen that the power of the $\alpha$-coverage parameter in terms of altering the behavior of the Cov protocols depends on the needs of the application: either use less energy and extend the lifetime by selecting fewer nodes, and thereby reducing the area of coverage; or use a great amount of energy and active nodes in order to obtain complete coverage of the area. A detailed analysis of the impact of the $\alpha$-coverage parameter in the lifetime is part of the future work and it is not included in this dissertation.

\subsubsection{Comparison with Distributed Protocols}

Two distributed protocols were selected to be compared with the A3Cov protocols: ACOS [85] and StanGA [88]. The second one is able to provide K-coverage, but the comparison will be focused on $K=1$. For the experiments with the distributed coverageoriented protocols, random scenarios were created based on the parameters described in the respective articles that presented the protocols. The figures presented in this section are reproductions of the results in their correspondent papers, which show in the $\mathrm{x}$-axis the number of active nodes produced by the protocols, and in the y-axis, the area covered by the reduced topology.

\subsubsection{Comparison With ACOS}

The scenarios used in [85] to test the performance of the ACOS protocol are based on a particular way of deployment: the authors assume that the area of deployment is divided 


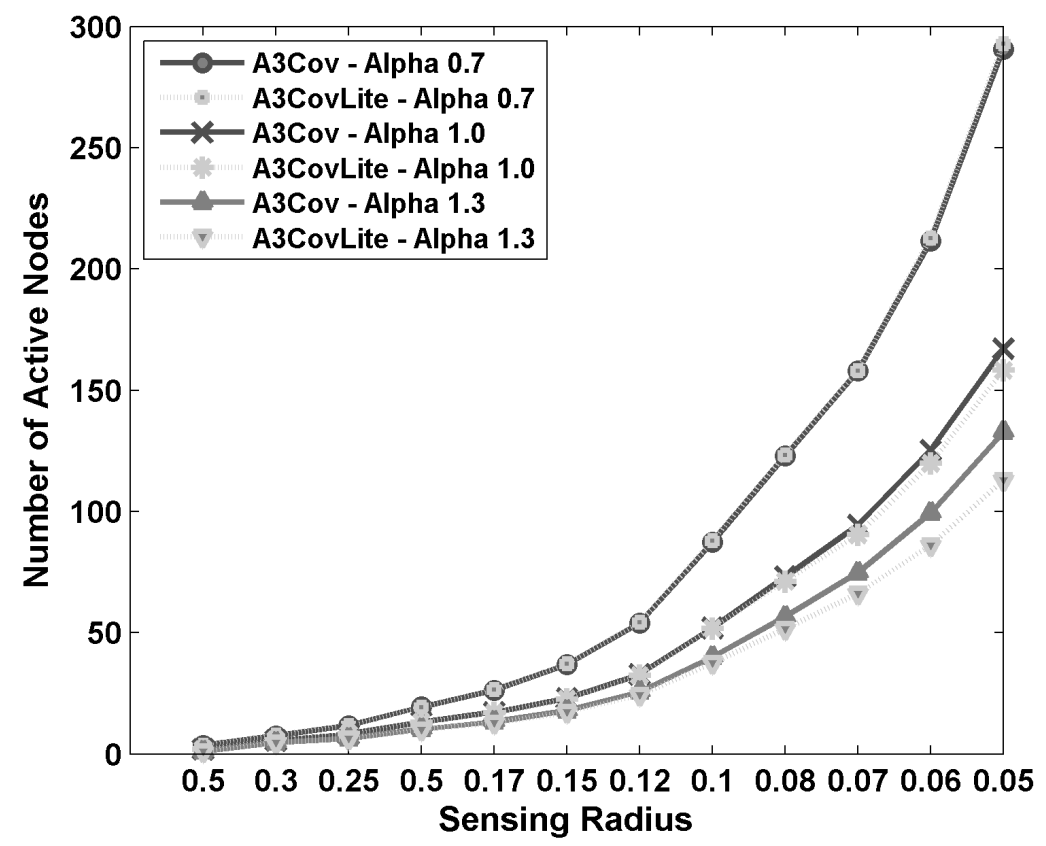

(a) Number of active nodes in sparse networks.

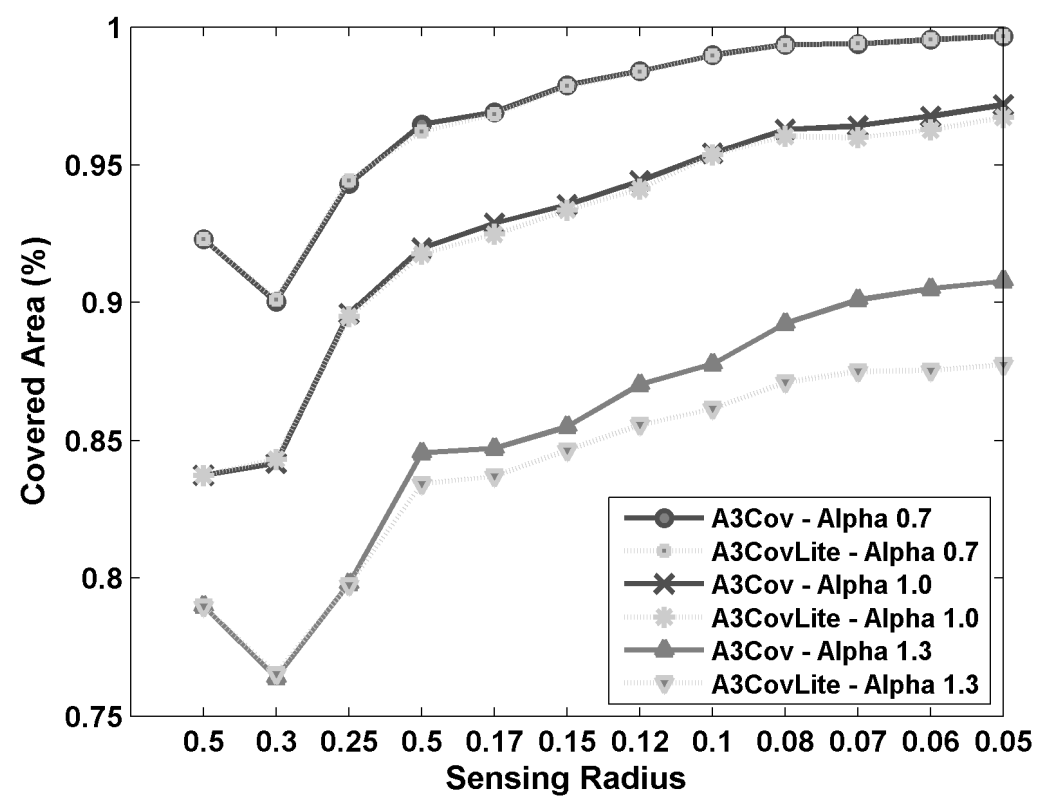

(b) Connectivity-coverage in sparse networks.

Figure 5.12: Performance of the A3Cov and A3CovLite protocols in sparse networks with different radii and $\alpha$-coverage parameter. 


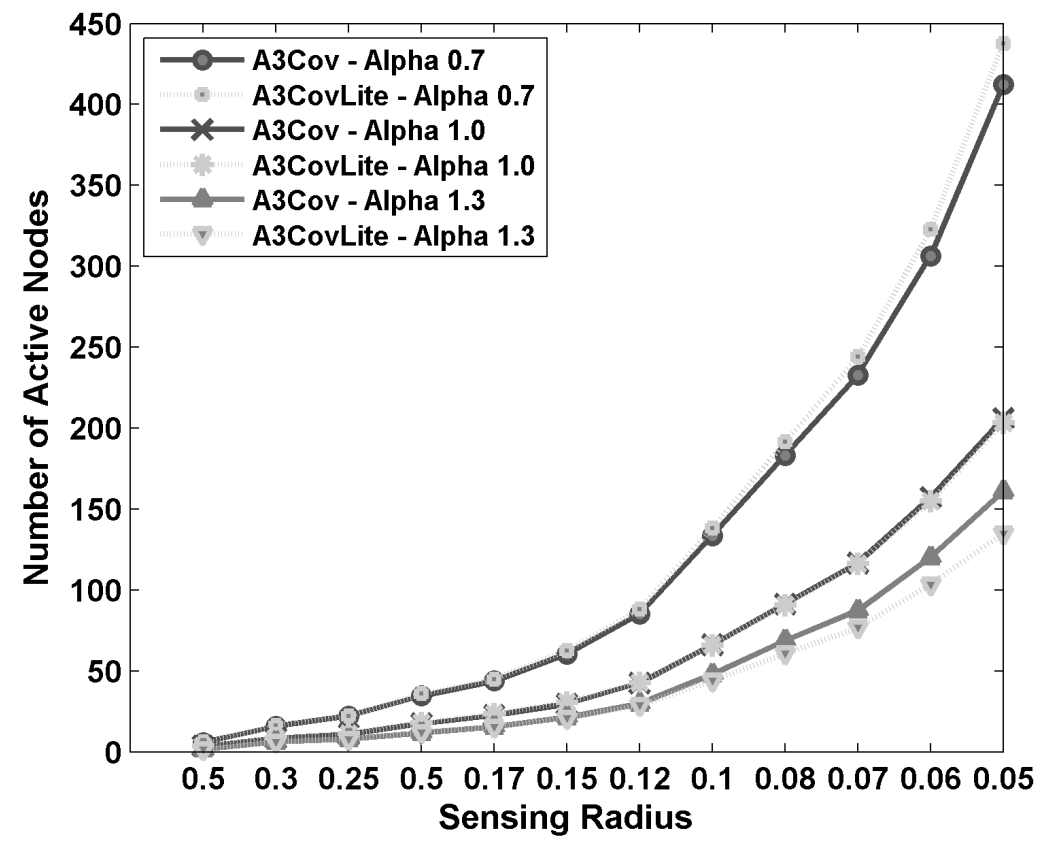

(a) Number of active nodes in dense networks.

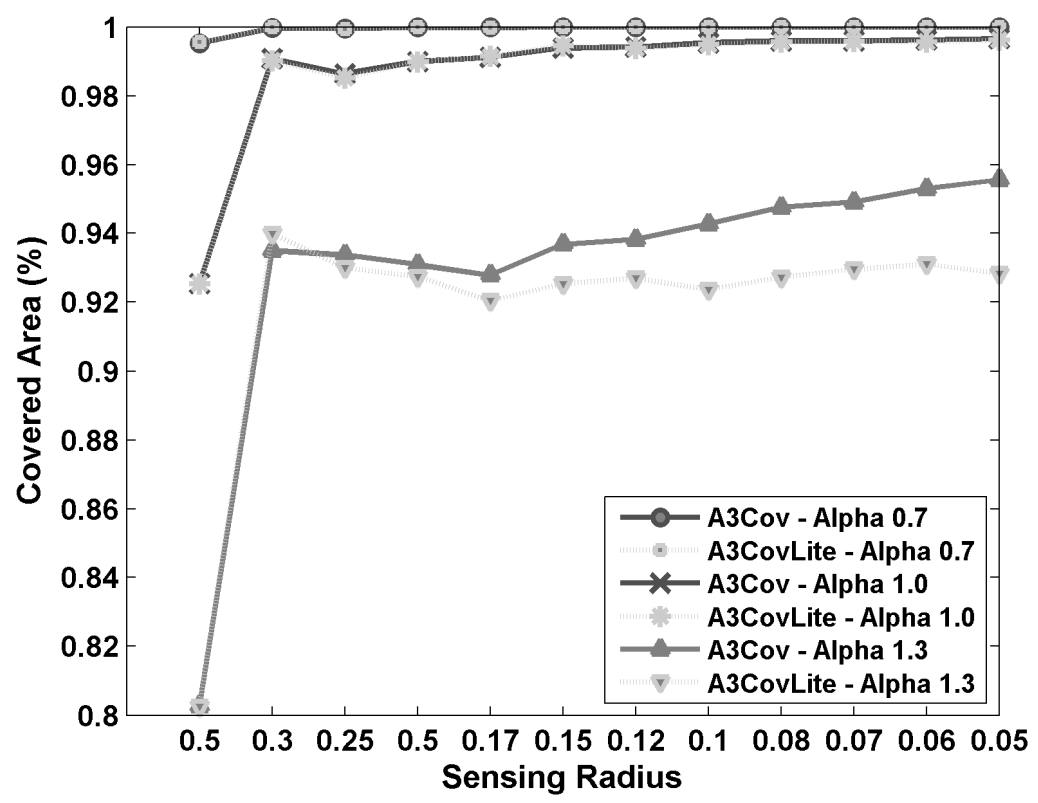

(b) Connectivity-coverage in dense networks.

Figure 5.13: Performance of the $\mathrm{A} 3 \mathrm{Cov}$ and $\mathrm{A} 3 \mathrm{CovLite}$ protocols in dense networks with different radii and $\alpha$-coverage parameter. 
in square cells of $R_{S} \times R_{S}$. Then in each cell, a constant number of nodes are deployed in a uniformly random manner. They work with 1,2, 4 and 8 nodes per cell. The scenarios the authors defined have an area of $400 m \times 400 m, R_{s}=20, R_{c}=40$, which with the different densities will produce the network sizes of 400, 800, 1600 and 3200, respectively. The evaluation in this section only considers the scenarios with 800 nodes.

Figure 5.14 is based on the original results shown in [85] for 800 nodes. The different points in the plotted function for ACOS correspond to the results in terms of number of active nodes and covered area, based on the variation of the $\varphi$ parameter, that represents the net coverage threshold in the protocol. The actual values of the parameter used in the experiments were not explicitly shown in the original article of ACOS, but they are most likely changing from 0.01 to 0.1 , with small increments, and from 0.1 to 1.0 with increments of 0.1. For the results of ACOS in this figure, only the values with a covered area greater that $65 \%$ were considered. The results of the $\mathrm{A} 3 \mathrm{Cov}$ and $\mathrm{A} 3 \mathrm{CovLite}$ protocols in this figure correspond to the results in terms of number of active nodes and area of coverage for $\alpha$-coverage parameter values from 0.5 to 1.5 .

As it has been seen, in all scenarios with a similar number of active nodes selected, $\mathrm{A} 3 \mathrm{Cov}$ and $\mathrm{A} 3 \mathrm{CovLite}$ offer a gain in ratio of covered area of about $10 \%$ over ACOS. Also, ACOS cannot reach a higher degree of coverage that $92 \%$, while the Cov protocols can reach practivally total coverage with the same number of nodes. ACOS can provide levels of coverage close to $100 \%$ only when the density of the netwoerk is very high (3200 nodes). It is expected that with higer densities, the Cov protocols will continue to produce even better coverage.

The performance of the Cov protocols can be related to the uniform-grid deployment of the nodes in the area. This characteristic produces a very evenly distributed topology, 


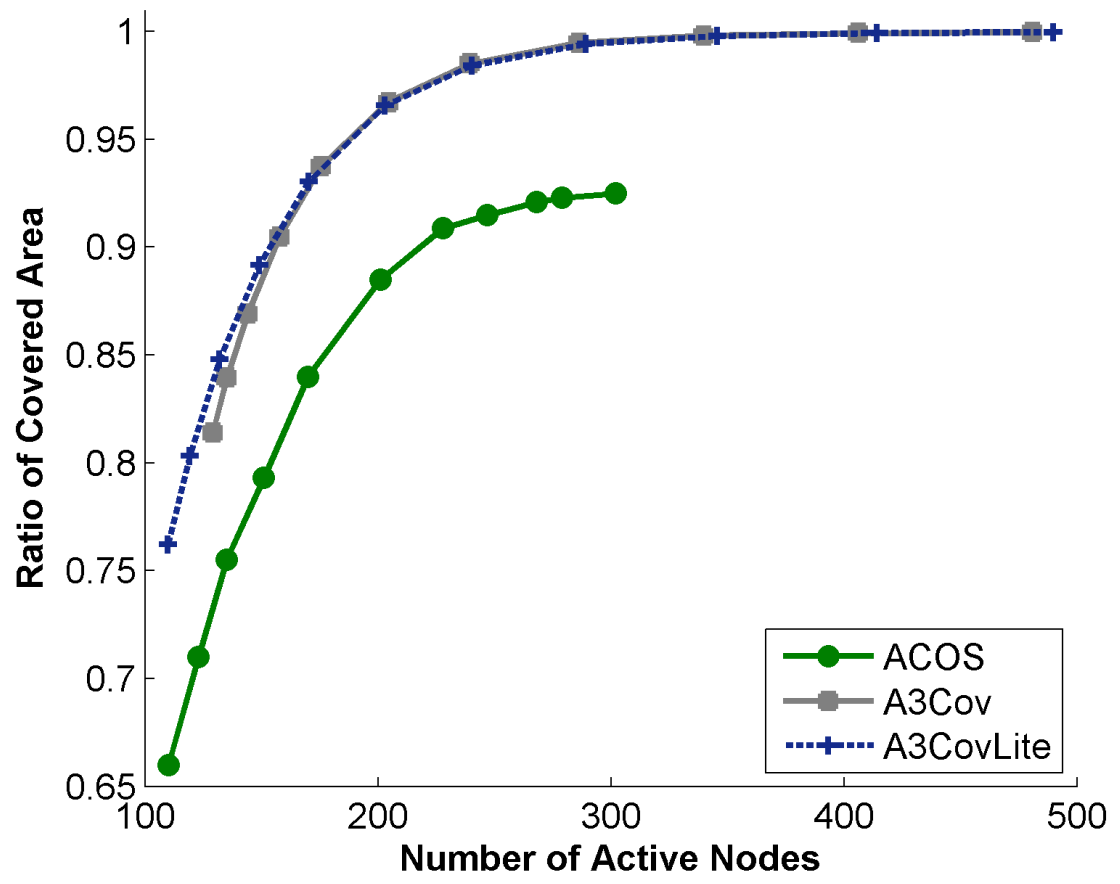

Figure 5.14: Comparison of performance of the $\mathrm{A} 3 \mathrm{Cov}, \mathrm{A} 3 \mathrm{CovLite}$ protocols and the ACOS protocol for 800 nodes. 
that allows the A3-based protocols to select a more spread out set of nodes to be active, reducing the overlapping of sensing areas, and thus, the need for selecting redundant nodes. In the case of ACOS, the protocol tries to guarantee complete area coverage and it pays special attention to covering small holes produced by the topology; this selection policy may increase unnecessarily the redundancy of the coverage, requiring ACOS to include a great amount of nodes, but not offering a great coverage ratio.

\subsubsection{Comparison With StanGA}

The scenarios used in [88] to test the performance of the StanGA protocol are defined by the following parameters: a uniformly random deployment of 200 nodes, a square deployment area of $50 \mathrm{~m} \times 50 \mathrm{~m}$, communication range of $R_{c}=20$, sensing range of $R_{S}=10$, and the $R_{S G}$ parameter changing from $1\left(0.1 R_{S}\right)$ to $12\left(1.2 R_{S}\right)$ with increment of 0.5 . In the case of $\mathrm{A} 3 \mathrm{Cov}$ and $\mathrm{A} 3 \mathrm{CovLite}$, the $\alpha$-coverage parameter was set to start at 0.1 , also with an increment of 0.5 . These values were selected in such a way that only levels of coverage greater that $93 \%$ of the area of interest were considered.

Figure 5.15, shows the results in terms of number of active nodes versus covered area for all the different configurations described. The points on each function correspond to the results when using the different values of their respective coverage parameters in decreasing order, when reading them from left to right. It can be seen that the lowest ratio of covered area occurs with the greatest value of the coverage parameters, and that the coverage increases when the coverage parameters decrease.

As it can be seen, the performance of the three protocols is very similar. This behavior is expected due to the similarities in the selection criteria of the nodes for coverage purposes. However, the main difference between the protocols is that the StanGA protocol 


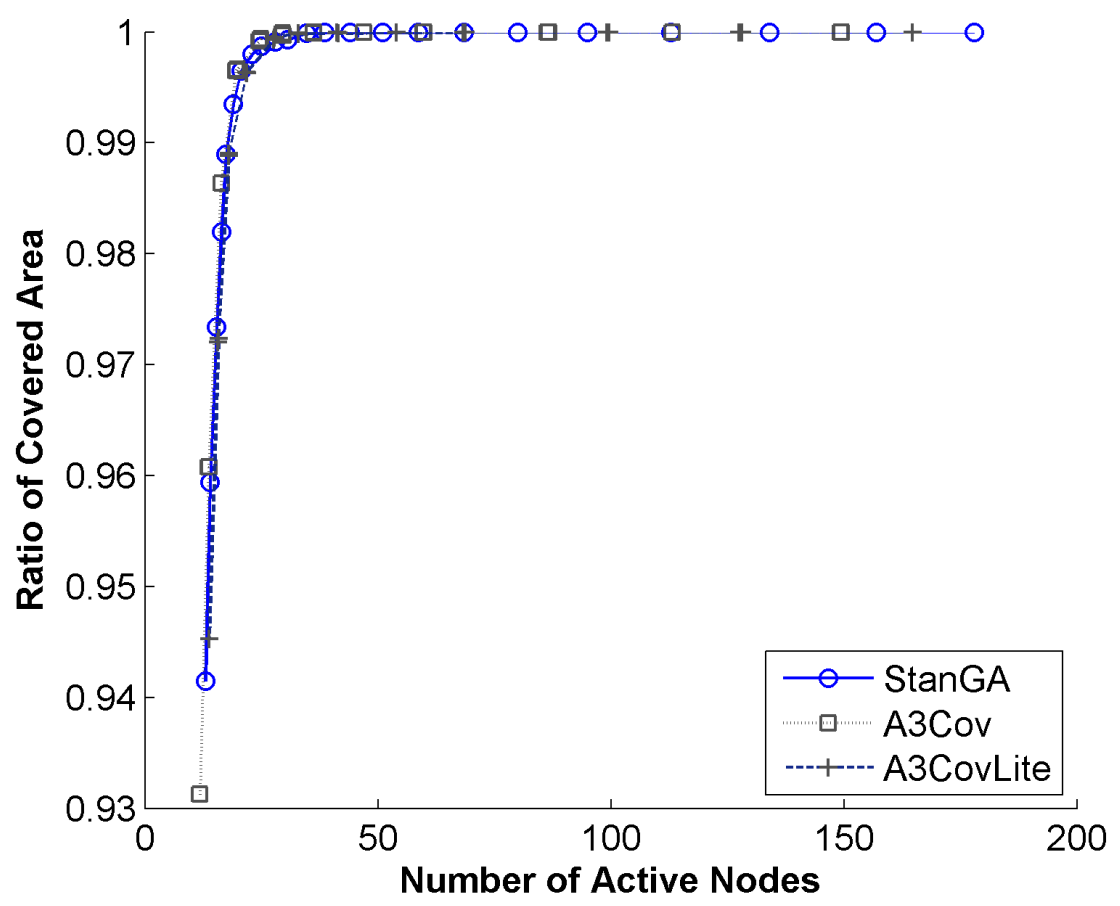

Figure 5.15: Comparison of performance of the $\mathrm{A} 3 \mathrm{Cov}$, $\mathrm{A} 3 \mathrm{CovLite}$ protocols and the StanGA protocol for different coverage configurations. 
cannot guarantee connectivity in the scenarios where $R_{S G}>R_{c \max } / 2$, as pointed out by the authors in their article. This is a clear disadvantage of this algorithm compared to the A3-based protocols which always guarantee a connected reduced topology that, in the case of the Cov protocols, is later extended to improve coverage. 


\section{Chapter 6: Topology Maintenance Protocols}

\subsection{Introduction}

This chapter is devoted to the proposed Topology Maintenance protocols, as part of the second component, after Topology Construction, to execute Topology Control. The main motivation for the study of Topology Maintenance as a separate problem from Topology Construction is the fact that most of the topology control algorithms proposed in literature are focused on the topology reduction portion, while they dedicate little or no effort to the maintenance policy of their algorithms; for example, most of the protocols define a new invocation of the topology construction when nodes start to fail. However, there is no analysis, theoretical or empirical, behind these decisions, so the optimal selection of the maintenance policy cannot not guaranteed.

The idea proposed in this section is that by separating the topology construction and maintenance protocols, and working based on a modular design, it is possible to test different combinations of these two kinds of protocols, in order to select, based on experimentation, the most appropriate maintenance policy for the reduced structure created by the construction protocol.

In this chapter, four topology maintenance algorithms will be introduced: Static Global Topology Rotation (SGTRot), Dynamic Global Topology Recreation (DGTRec), Hybrid

Global Topology Recreation and Rotation (SGTRecRot) and Dynamic Local DSR (DL- 
DSR), based on the Dynamic Source Routing (DSR) protocol. The first two have been used in literature but not implemented as independent protocols, and the final two are completely new protocols.

The performance of the four proposed topology control protocols will be tested, working jointly with two topology construction protocols: A3, EECDS and CDS-Rule-K. In addition, a sensitivity analysis of performance is included, based on the parameters of energy threshold, inter-reset time and network density.

\subsection{Static Global Topology Rotation}

The Static Global Topology Rotation (SGTRot) is the implementation of a protocol for rotating multiple reduced topologies. The main assumption that this protocol makes is that each node keeps track of several Virtual Network Interfaces, or VNI. These VNIs contain information relevant for a node concerning each separate reduced topology created in the network: address, role(active, inactive or sink), communication and sensing radius (in the cases where these are variables), routing information, etc. In addition, in the occurrence of a rotation, each node should be able to change the currently active VNI in a short period of time.

The protocol is very simple. Assume that the topology maintenance trigger is activated in one of the nodes. It will send a Notification Message to the sink node, informing that its trigger went off. When this message arrives to the sink node, it will decide if the occurrence of the triggering event is enough of a reason to start the rotation process. If that is not the case, the sink node will ignore the message. In some cases the sink is the only node responsible for the triggering of the maintenance procedure, like when it depends on a local decision or a local timer. 
In the case where the sink considers that a rotation is necessary, the sink will determine the id of the next available VNI, will send a Rotation Message including the id of the next VNI, and it will change its current active VNI to the next one, updating all the relevant information about the "new" network. Once this message arrives at a node, the node will forward the Rotation Message and will change also its VNI. After changing to the new VNI, a node will not forward any more Rotation Messages with the id of the current VNI, which produces a controlled flooding in which every node transmits the Rotation Message just once.

After every rotation, the sink will evaluate if it has at least one active node in its neighborhood. If the sink finds at least one, then the VNI will be active until a new triggering event occurs. In the case where the sink is isolated from active nodes, then it will eliminate the current VNI and will start another rotation to the next VNI. The network is finally dead when there are no VNIs available to perform a rotation. The current version of this protocol does not include mechanisms to verify the status of connectivity and coverage of the new VNI, or that all the nodes were rotated, but it has been considered for future new versions of this protocol.

\subsection{Dynamic Global Topology Recreation}

The Dynamic Global Topology Recreation (DGTRec) is perhaps the most commonly used maintenance policy in the literature of topology control, mainly because it is the simplest one to implement because it does not require any pre-calculated information and because it is tightly related to the topology construction protocols, which have been the main focus of research in this area. 
As in the SGTRot protocol, the topology maintenance trigger is activated in the sink itself or in one of the nodes. It the triggering event happened in a regular node it will send a Notification Message to the sink node, informing that its trigger went off. When this message arrives at the sink node, it will decide if the occurrence of the triggering event in enough reason to start the rotation process. If that is not the case, the sink node will ignore the message.

In the case where the sink considers that a rotation is necessary, the sink will send a Reset Message and, if it is the only sink in the topology, it will schedule a new execution of the topology construction protocol it used in the first place to reduce the topology. When a node receives a Reset Message, it will forward it immediately to all its neighbors, and then the node will eliminate all information about the current reduced topology, and will move itself into the initial state of the topology construction protocol, waiting for the new execution. As in the SGTRot, a controlled flooding is in charge of reseting the entire network.

\subsection{Dynamic Local - DSR}

This protocol is a new implementation of an energy-based dynamic local topology maintenance technique. The DL-DSR is based on the well-known Dynamic Source Routing (DSR) [108] protocol for wireless ad hoc networks. This protocol was designed to allow a node with very low remaining energy to find replacements that will offer connectivity to its soon-to-be-orphaned children.

The protocol works in two phases. During the first phase, the affected node, $N_{i}$, i.e. the node whose energy level is below a certain threshold, broadcasts a WakeUp Message that includes the list of all its children nodes. This message will reach all its children $C_{i}$, both 
active and sleeping, which after receiving the message change their status to Semi-Active. These nodes will send a WakeUp-3 Message to their neighbors and children, which, upon receiving the message, will turn themselves on and change their status to TM-Initial, which leaves them ready for the execution of the local maintenance protocol. The WakeUp Message is also heard by $N_{i}$ 's active parent $P_{i}$ and its other children $B_{i}$ (brothers of $N_{i}$ ), who were part of the reduced tree. Upon receiving the WakeUp Message, $P_{i}$ stores the list of children of $N_{i}$ and then, with the brother nodes $B_{i}$, broadcasts a WakeUp-2 Message among their respective children and neighbors to wake them up too. All the nodes that received the WakeUp-2 Message will change their status to TM-Initial and will send a WakeUp-3 Message to their respective neighborhoods. The nodes that received the WakeUp-3 Message will not forward any other message, which will contain the process to an area defined by all nodes two hops away on $N_{i}$ 's children's side, and three hops away on its parent's side. These node are in Semi-Active or TM-Initial states. Each node can be awakened only once, and will ignore any other Wake-Up message. This procedure is done in this manner to guarantee that algorithms like A3, which builds trees with very spread out branches, can very likely find alternate paths to restore the topology, while covering all the nodes that were previously covered by node $N_{i}$. Figure 6.1 shows an example of the topology after executing the first phase of the DSR-based dynamic local topology maintenance technique just described, showing the area of the network that has been awakened to run the maintenance procedure.

The second phase of the protocol begins with the parent node $P_{i}$ doing a restricted flooding of a Route Request Message. The flood is restricted because it has a final destination: all semi-active nodes only, which are the new orphan nodes from $N_{i}$. The Route Request Message includes a data structure that contains the list of node $N_{i}$ 's children that are now 


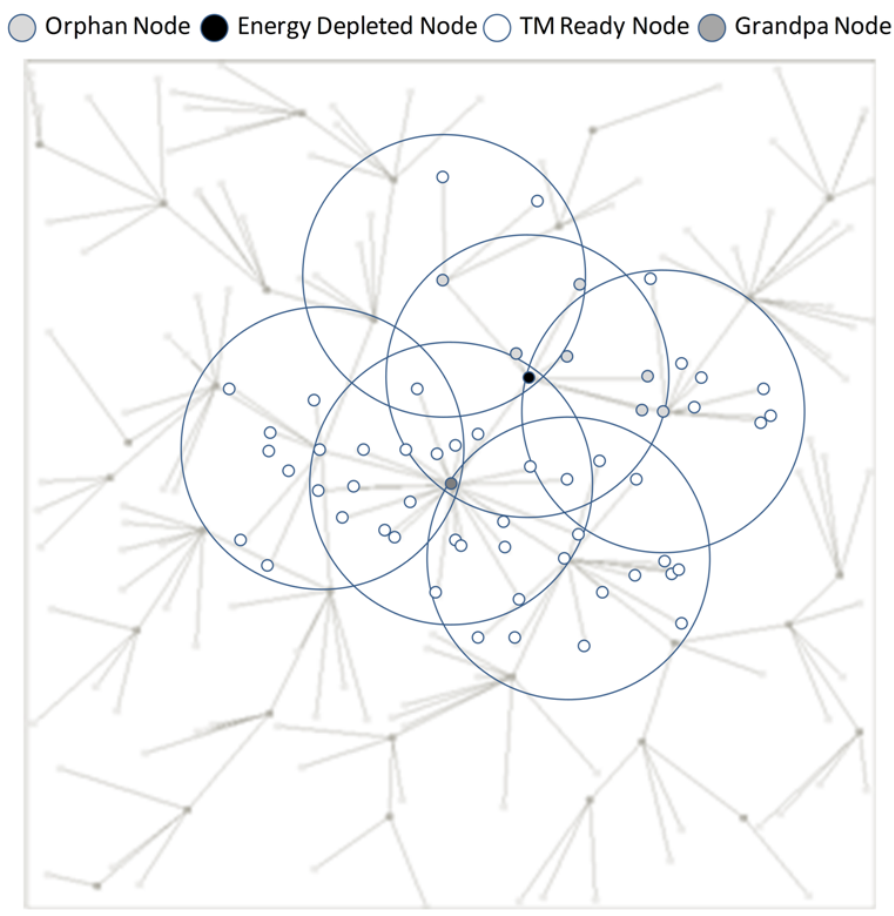

Figure 6.1: Phase one of the DSR-based dynamic local topology maintenance technique.

disconnected from the network as well as the current routing and energy-distance information of the path that the message has gone through. Upon receiving a Route Request Message for the first time, the receiving node registers the current path information contained in the message and sets a timeout in order to wait for more messages of this kind. Every time the node receives a new Route Request Message, it will compare the path from the new message with the best path it has found yet. If the new path is better, then the node will update the path; otherwise, the message will be ignored.

Once the timeout has expired, the node will update the information of the best registered path with its own cost in length and energy, and will send a Route Request Message, including new path information. Also, the node will change its status to TM-Ready. A node in this state will ignore every Route Request Message and will set a timeout in order to 
wait for replies on the route requests. If this timeout expires, then the node will return to its normal role in the reduced topology (active or sleeping mode).

It is expected that these messages will eventually reach at least one of the nodes $C_{i}$. These nodes, just as the non-orphan nodes, will set a timeout in order to wait for the arrival of more Route Request Messages from different paths; however, the timeout in these nodes will be longer in order to allow a higher number of possibilities. Once the timeout expires, the $C_{i}$ nodes select the best route back to the parent node $P_{i}$ based on the route information contained in the messages, and will include this information in a Route Reply Message, which is a unicast message addressed to the previous hop in the path. Receiving a Route Reply Message means that the specific node has been selected as part of the new path to the orphan node. The receiver node will change its status to TM-Active, and will forward the message to the previous hop on the best path it has registered for the orphan node. A single node can be selected to be part of different paths.

This process continues until $P_{i}$ receives the Route Reply Messages from all the $C_{i}$ nodes that have been reached by the protocol. As the timeout to return to normality expires, all nodes that were not selected to be part of a new path will return to their original roles: sleeping or active nodes. In the case that a node was selected as part of a new path, no matter which role it had, the node will remain active in order to guarantee connectivity.

\subsection{Hybrid Global Topology Recreation and Rotation}

The Hybrid Global Topology Recreation and Rotation (HGTRecRot) protocol is a mixture between the static and dynamic global approaches presented here. As a static protocol, it assumes that the topology construction protocol generates more than one VNI, which will be rotated, as in the SGTRot protocol, based on the triggered event from the 
nodes in the network. The main difference from the static protocol comes when the sink detects that it has become isolated from the network, due to lack of active neighbor nodes. At this point, the sink sends a Reset Message that invokes the topology construction protocol in order to renew the current VNI, as in the DGTRec protocol. If the sink is still isolated after the recreation process, then the sink will eliminate the current VNI from the list of available ones and will rotate to the next one. If there are no more VNIs available, then the sink will determine that the network is dead.

\subsection{Performance Evaluation}

This section includes common information about the simulation-based performance evaluation that will be carried out in the next three chapters to assess the performance of the various static, dynamic, and hybrid topology maintenance techniques. The purpose of the experiments is to determine the benefit of implementing topology maintenance techniques in both sparse and dense networks, and compare their performance versus the choice of not implementing topology maintenance at all.

In these experiments, sparse topologies are defined as topologies in which the communication radius is calculated based on the Critical Transmission Range (CTR) formula of Penrose-Santi [4] (Equation 2.1 described in Section 2.2.1.1). This guarantees that the node degree is very low, creating a weakly-connected topology. Dense topologies are defined as sparse topologies in which the original number of nodes is doubled. Each point in the graphs is the average of running the same experiment 150 times, i.e. with 150 different topologies.

In all simulations the A3, EECDS, and CDS-Rule-K topology construction algorithms described in Chapters 2 and 4 were utilized. The implementations were coded and tested 
Table 6.1: Simulation parameters for topology maintenance protocols.

\begin{tabular}{|c|c|c|}
\hline & Sparse Topologies & Dense Topologies \\
\hline Deployment area & \multicolumn{2}{|c|}{$200 \mathrm{~m} \times 200 \mathrm{~m}$} \\
\hline Number of nodes & 50 & 100 and 400 \\
\hline Number of sinks & \multicolumn{2}{|c|}{1 sink } \\
\hline $\begin{array}{l}\text { Number of topologies (runs) } \\
\text { per experiment }\end{array}$ & \multicolumn{2}{|c|}{150} \\
\hline Transmission Range & \multicolumn{2}{|c|}{ 1xCTR(50) equivalent to: $37 \mathrm{~m}$ ( [4]) } \\
\hline Node Distribution & \multicolumn{2}{|c|}{ Uniform $(200,200)$} \\
\hline Time Threshold & \multicolumn{2}{|c|}{1000 time units } \\
\hline Energy Threshold & \multicolumn{2}{|c|}{$10 \%$ of total energy } \\
\hline $\begin{array}{l}\text { Max number of reduced topologies } \\
\text { (static and hybrid schemes) }\end{array}$ & \multicolumn{2}{|c|}{3 reduced topologies } \\
\hline$E_{\max }$ & \multicolumn{2}{|c|}{1 Joule } \\
\hline A3 Weights & \multicolumn{2}{|c|}{$W_{E}=0.5, W_{D}=0.5$} \\
\hline
\end{tabular}

in the Atarraya simulation tool. In all simulations, the wireless sensor devices are uniformly distributed in an area of interest of $200 m \times 200 m$; the number of nodes is varied to create sparse and dense networks; and all scenarios have one sink. Each active node in the current reduced topology is scheduled to send data messages directed to the sink every 10 time units. Since all topology construction algorithms produce a tree-based reduced topology rooted at the sink, a very simple routing algorithm is implemented in which nodes forward the data messages to their respective parents. In all experiments, no data aggregation or similar strategy is implemented. Energy is drained when a packet is either sent or received according to the energy dissipation model presented in Section 3.3.4. Table 6.1 includes a summary of the most important simulation parameters used in all simulations. 


\subsubsection{Performance Evaluation of Static Global Topology Maintenance Techniques}

This section presents a performance evaluation of the static global topology rotation technique carried out in the simulation tool Atarraya, using the simulation parameters and assumptions described in the Table 6.1. The purpose of the experiments in this section is to determine the benefit of implementing static global topology maintenance techniques in both sparse and dense networks, and compare their performance versus the choice of not implementing topology maintenance at all. The following topology maintenance techniques and triggering criteria are included:

- No Topology Maintenance - No TM: The initial reduced topology works permanently until the sink detects that it does not have any more active nodes in range. In general, this is the same termination policy for all the algorithms.

- Static Global Time-based Topology Rotation - SGTTRot: Every pre-determined time interval the topology maintenance algorithm rotates the active reduced topology for one of the pre-planned ones.

- Static Global Energy-based Topology Rotation - SGETRot: Every time a node reaches a critical energy threshold, the topology maintenance algorithm rotates the active reduced topology for one of the pre-planned ones.

For simplicity, in the experiments, the number of pre-planned topologies is limited to three $^{1}$. The process of selecting these three topologies is different depending on the underlying topology construction mechanism. In the case of A3, its selection metric is manipulated to lower the probability of selecting one node in more than one topology. However, since the same topology construction algorithm is run every time, if a particular

\footnotetext{
${ }^{1} \mathrm{~A}$ more complex mechanism that finds as many as possible completely disjoint topologies can be found in [69].
} 
node that has been used before in another topology is needed to guarantee network connectivity, it may be selected again. In other words, the static global technique based on A3 may produce shared-disjoint topologies.

In the case of the EECDS topology construction algorithm, it also uses a numerical metric to perform the selection of the active nodes, which allows the application of a penalty in this metric to all nodes that have been selected to be active in other subsets. However, depending on the density of the network, the metric cannot be changed in all the nodes. EECDS works in two phases, selecting Black nodes in the first phase, and Blue and Gray nodes in the second phase. Black nodes are backbone nodes that act as clusterheads, Blue and Gray nodes are then used to interconnect the clusterheads. As a result of this twophase approach, EECDS can only reduce the selection metric to the Black nodes that have been selected previously to be active in other subsets. In dense topologies it is expected that if there is a change in the selection metric of the Black nodes, the set of elected Gray nodes will also change. However, in sparse topologies the case is different because there are so few possibilities of selection of Gray nodes that they will tend to be the same ones in every subset.

Finally, in the case of the CDS-Rule-K topology construction algorithm, since it does not include any numerical metric to select the nodes, the algorithm is left unchanged, meaning that it may produce very similar trees.

\subsubsection{Sparse Networks}

Figure 6.2 shows network lifetime simulation results when performing static global topology maintenance in sparse networks. Two common observations can be made. First, static global topology maintenance in sparse networks can either improve or degrade the 
network lifetime compared with the option of not performing topology maintenance at all. Second, the performance is not significantly better or worse, meaning that applying static global topology maintenance techniques in sparse networks might not be useful.

These results are totally expected, as in sparse networks not many disjoint topologies can be created. Remember that in this scenario only 50 nodes are spread in an area of 200 square meters. This means that the topology maintenance procedure just activates the same topology, or a very similar reduced topology, every time and the overhead related to the changing process drains extra energy, killing the nodes earlier. The impact of not having disjoint subsets can be appreciated in the CDS-Rule-K algorithm (Figure 6.2(c)), as both static techniques did not even reach the performance of having no topology maintenance at all.

Another important observation is that the performance of the static global techniques may change according to the value of the triggering mechanism, i.e., the value of the timer and the energy threshold. Nonetheless, it is not expected that changing these values to more appropriate (optimal) ones will produce considerably better or worse results, as the effect of the reduced number of disjoint topologies will prevail over the threshold values. A sensitivity analysis looking at the effect of these values in the performance of the techniques is included in Section 6.8 for dense networks.

Finally, Figure 6.6.1.1 compares the best performing techniques for each algorithm. As it can be observed, all energy-based techniques perform better than the time-based techniques. This result is expected as the energy threshold does not make the topology to change until the nodes are almost without energy. Since there are not many disjoint topologies, the energy-based techniques do not waste as much energy as the time-based techniques in the switching process, which switch the topology every 1000 time units. Of the three techniques, the one based on the A3 algorithm is the best performing. This is 


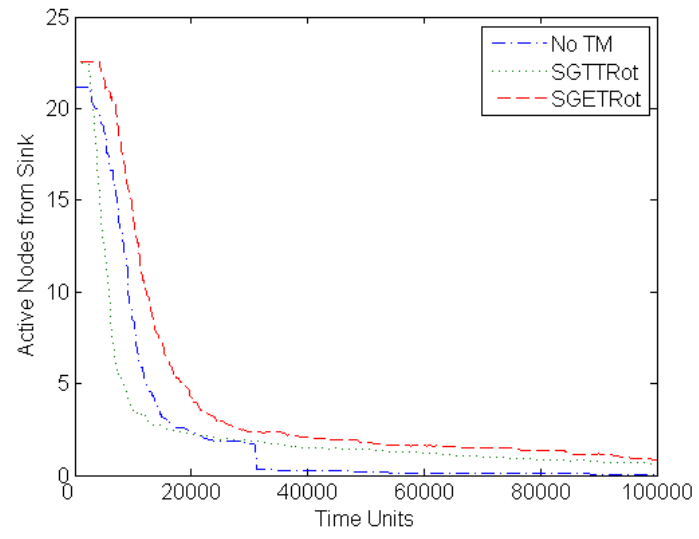

(a) A3

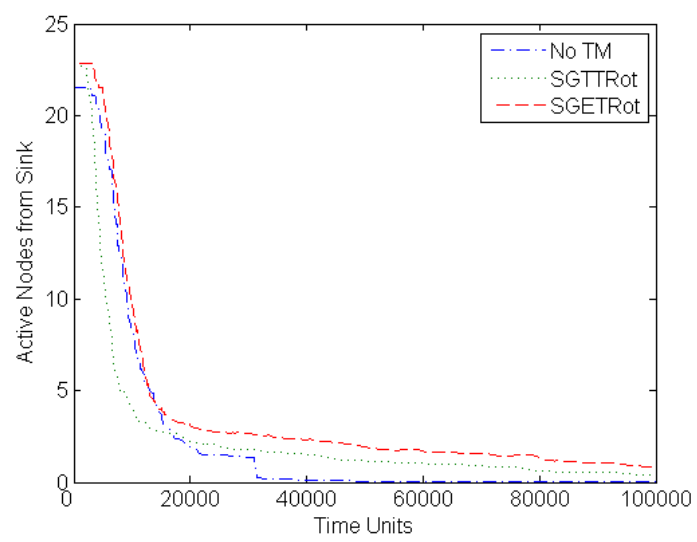

(b) EECDS.

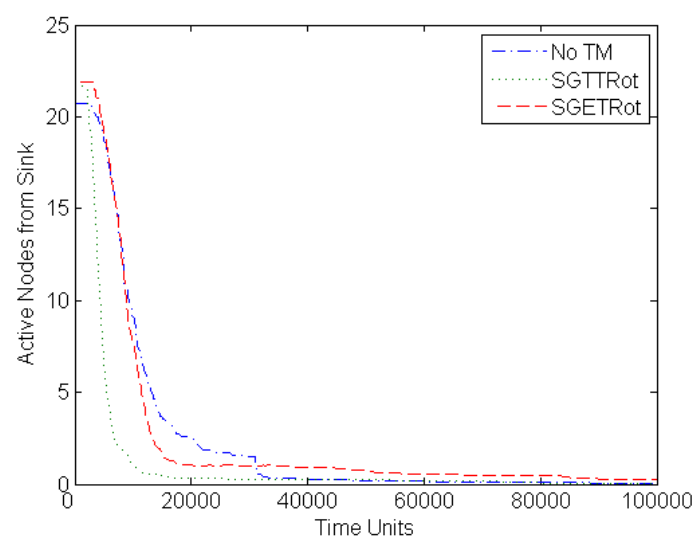

(c) CDS-Rule-K

Figure 6.2: Network lifetime with and without static global topology maintenance using the A3, EECDS, and CDS-Rule-K topology construction mechanisms in sparse networks. 


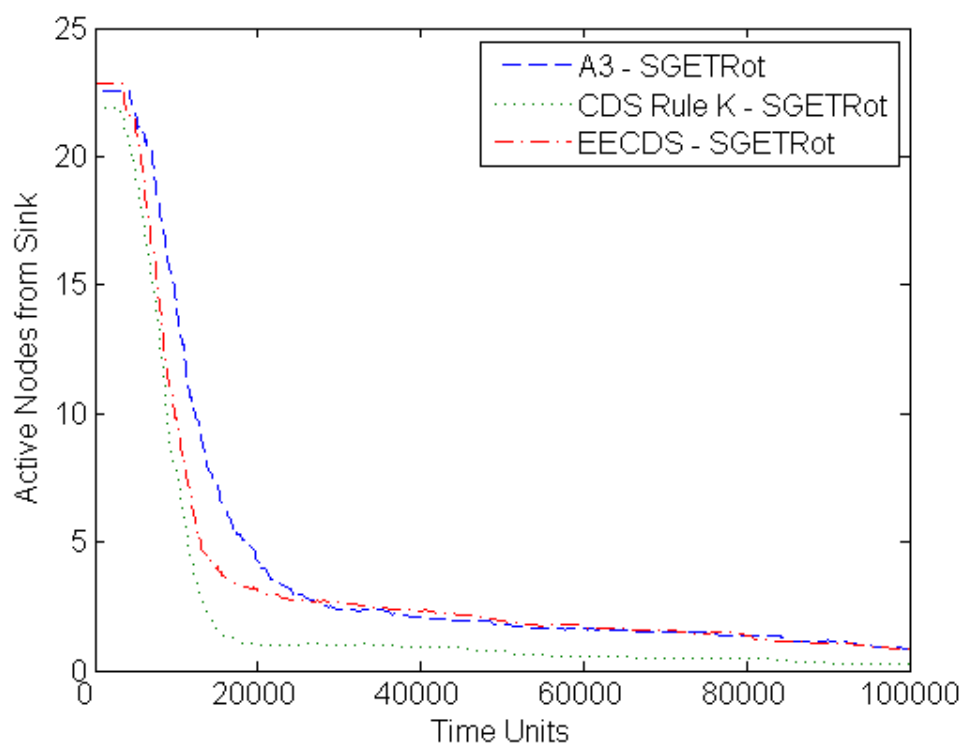

Figure 6.3: Best performing static global topology maintenance techniques in sparse networks.

because A3 is more energy efficient than EECDS and CDS-Rule-K, as it has been demonstrated in [106].

The results of all these experiments are surprising at first glance since it was expected that somehow topology maintenance had to provide some sort of improvement. However, this is not the case. For all topology construction algorithms, a static global topology maintenance technique decreases the network lifetime. The explanation of this has to do with two related factors. In a sparse network not many (if more than one) completely disjoint topologies can be built, reducing the case to the simple case of not having topology maintenance at all. In the case of topologies with shared nodes, although more than one topologies can be created, many of them (if not all) share the same critical nodes, then reducing the energy even further because of the repetition of the topology maintenance process. The time-based scheme performs better than the energy-based technique because the former gives more topologies the change to use those critical nodes. In the latter case, 
the first topology uses those critical nodes almost until they are depleted. Of course this is a consequence of the energy threshold used in the simulations, in which the nodes ask the sink to change the topology when they have consumed $90 \%$ of their total energy. Better results might be obtained with higher thresholds.

\subsubsection{Dense Networks}

The performance of the static global topology maintenance techniques is expected to improve with the network density, as it increases the chance of having more node-disjoint topologies. This improvement in performance is, in fact, shown in Figure 6.4, which includes the results for more dense networks, those using 100 nodes instead of 50. Comparing Figures 6.2 and 6.4, it can be observed that while in sparse networks the no topology maintenance option was either the best performing one, or very close to be the best performing one, in dense networks the opposite is true. Actually, it can be seen from the figures that all topology maintenance techniques under consideration extend the network lifetime over the no topology maintenance option, so their use is more than justified. Two additional observations are worth including. First, the performance of the topology maintenance techniques depends on the underlying topology construction algorithm. It can be seen that while the performance of the A3-based and EECDS-based techniques improved with respect to the same experiments in sparse networks, the performance improvement of the CDS-Rule-K-based technique is not as big. This has to do with the way of selecting the disjoint trees, as explained at the beginning of this section. A3 and EECDS allow the manipulation of the node selection metric, and, therefore, they are able to build more node-disjoint trees. Second, as in the case of sparse networks, the techniques that used the energy triggering metric outperformed the time-based techniques. 
Again, this has to do with choosing an optimal switching time or energy threshold, which has not been attempted thus far. A sensitivity analysis is included in Section 6.8, which also looks at the effect of increasing the network density even further.

\subsubsection{Performance Evaluation of Dynamic Global Topology Maintenance Tech- niques}

Dynamic global topology maintenance techniques change the entire topology by running the topology construction mechanism anew every time it is needed. Using the same network parameters described in Table 6.1, simulation experiments were carried out to assess the network lifetime with dynamic global topology maintenance techniques in sparse and dense networks using the A3, EECDS, and CDS-Rule-K topology construction algorithms compared against the case where no topology maintenance is performed. The following topology maintenance techniques and triggering criteria are included:

- No Topology Maintenance - No TM: The initial reduced topology works permanently until the sink detects that it does not have any more active nodes in range. This is the same termination policy for the rest of the algorithms.

- Dynamic Global Time-based Topology Recreation - DGTTRec: Every set time interval the topology maintenance algorithm terminates the previous reduced topology and invokes the topology construction algorithm to create a new one.

- Dynamic Global Energy-based Topology Recreation - DGETRec: Every time a node reaches a critical energy threshold, the topology maintenance algorithm terminates the previous reduced topology and invokes the topology construction algorithm to create a new one. 


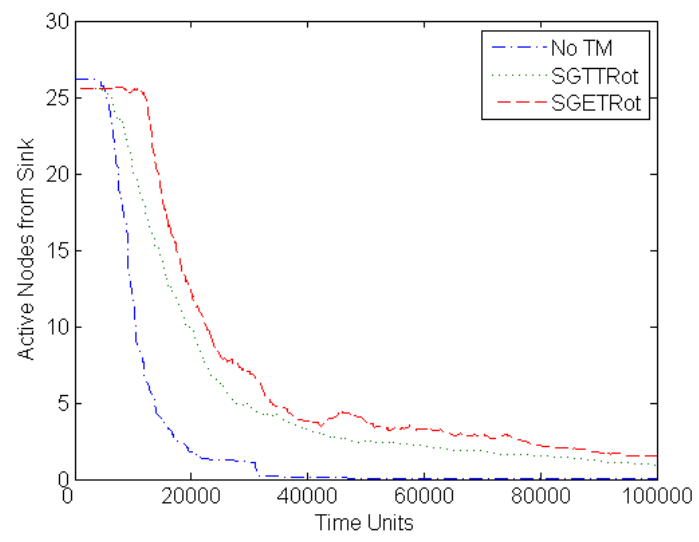

(a) $\mathrm{A} 3$

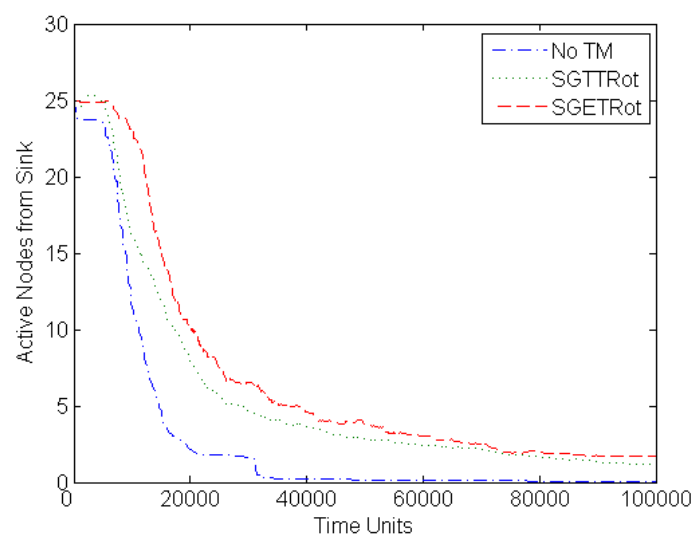

(b) EECDS.

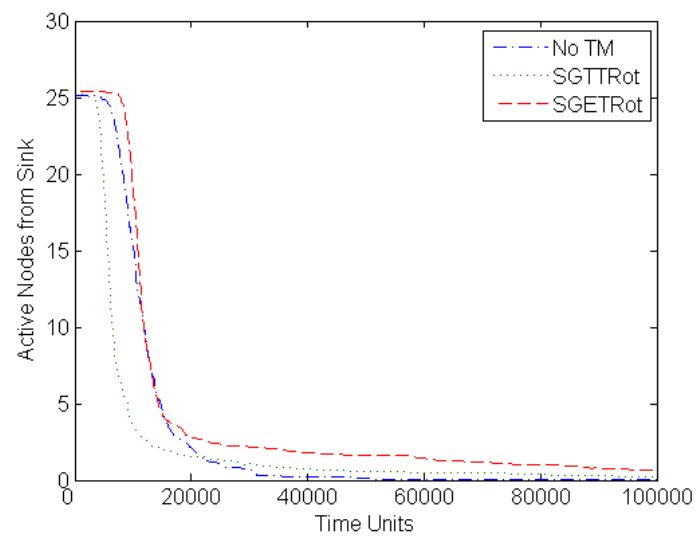

(c) CDS-Rule-K

Figure 6.4: Network lifetime with and without static global topology maintenance using the A3, EECDS, and CDS-Rule-K topology construction mechanisms in dense networks. 


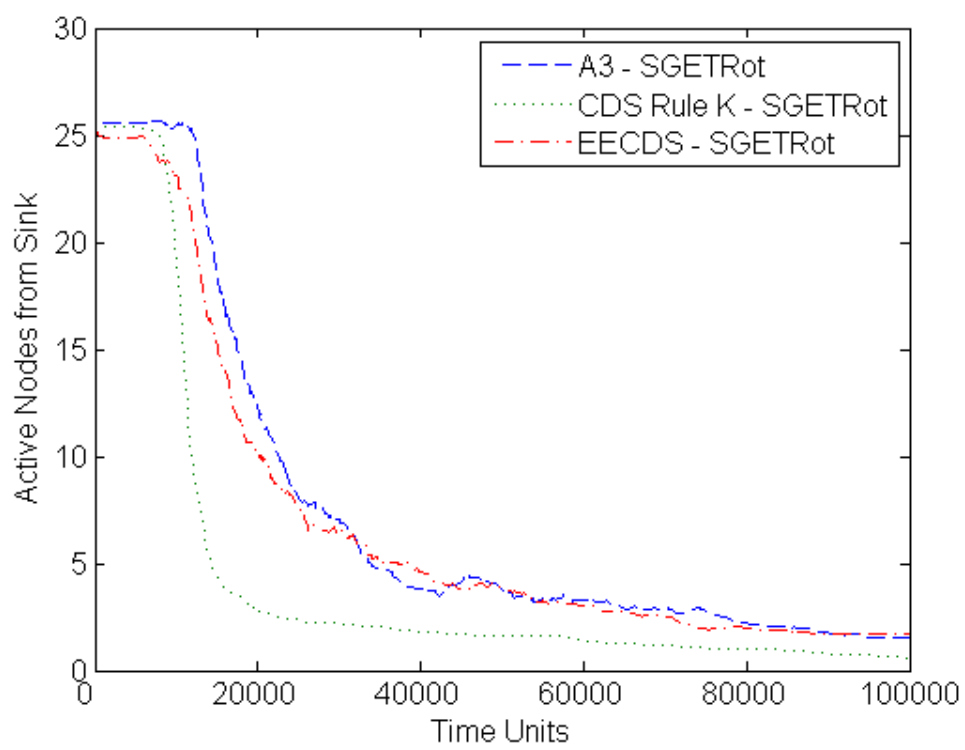

Figure 6.5: Best performing static global topology maintenance techniques in dense networks.

\subsubsection{Sparse Networks}

Figure 6.6 shows the network lifetime simulation results using dynamic global topology maintenance techniques in sparse networks using energy and time triggering criteria. Contrary to the static global technique results for sparse networks shown in Figure 6.2, now all topology maintenance techniques provide better performance than the case with no topology maintenance. It is clear that the new topology construction process is able to find better topologies than the pre-planned algorithms, extending the network lifetime, even in sparse networks. However, the performance improvement over the no topology maintenance option is not considerable either, reinforcing the conclusion reached before that, in sparse networks, topology maintenance does not provide important benefits. Comparing Figures 6.6 and 6.2 again, it can be observed that dynamic techniques provide better performance than static ones, which is expected given that more and better topologies 
can be built using dynamic methods. A more detailed comparison among these techniques is included in Section 6.7.

As in the case of static global techniques, from Figure 6.6.2.1, the same two conclusions can be drawn. First, all energy-based techniques outperformed the time-based techniques. Again, these performance results might vary depending on the time period and the energy threshold chosen. Similarly, in the case of A3, the behavior can also be changed varying the weights in Equation 4.1, which would give preference to those nodes that contain more energy, or those nodes that are closer to the parent node. There are clear trade offs using these variables and weights, which are beyond the scope of the book at this time. Second, the technique using the A3 topology construction algorithm is the best performing one, although not by an important margin. This is due to A3's lower and linear message complexity compared with EECDS and CDS-Rule-K [106].

\subsubsection{Dense Networks}

More interesting results are obtained in the case of dense networks, as can be observed from Figure 6.8. Now the figure shows that by increasing the number of nodes from 50 to 100 , dynamic global topology maintenance techniques are able to keep active a considerably higher number of nodes compared with the option of no topology maintenance. Compared with the case of dynamic global techniques in sparse networks shown in Figure 6.6, the performance in dense networks is nearly twice as good as the performance in sparse networks, meaning that more and better trees can be found in dense networks. Also, as in the case of static global techniques in dense networks, here the performance provided by the energy-based techniques outperformed the ones based on time. As stated before, this does not mean that time-based techniques are better or worse than energy- 


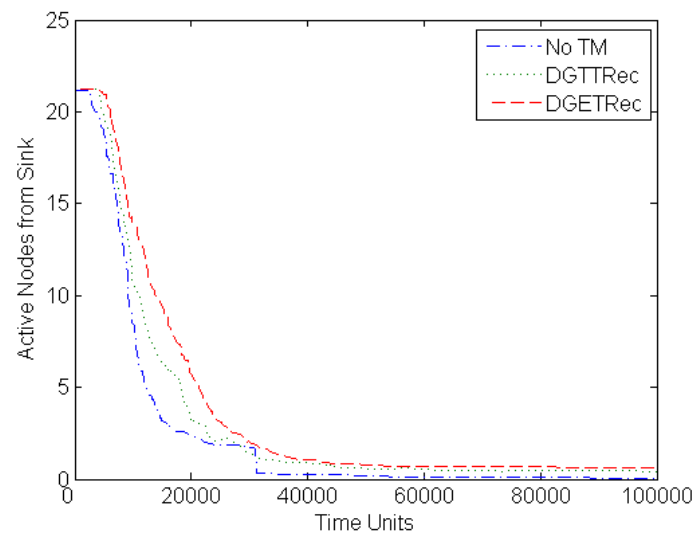

(a) $\mathrm{A} 3$

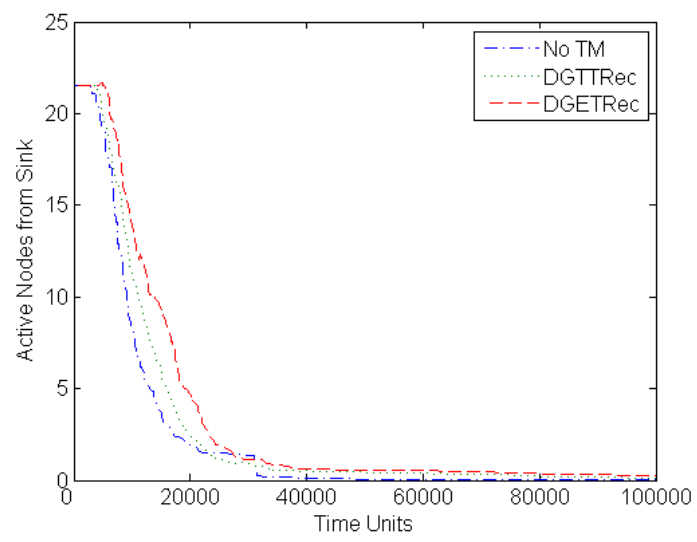

(b) EECDS.

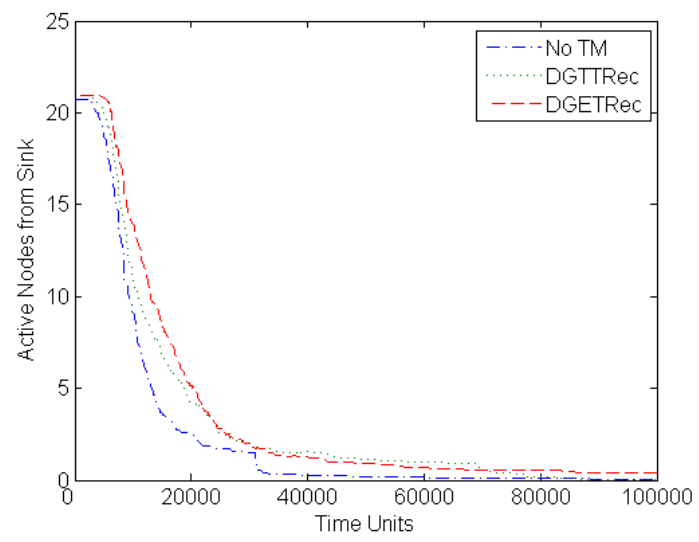

(c) CDS-Rule-K

Figure 6.6: Network lifetime with and without dynamic global topology maintenance using the A3, EECDS, and CDS-Rule-K topology construction mechanisms in sparse networks. 


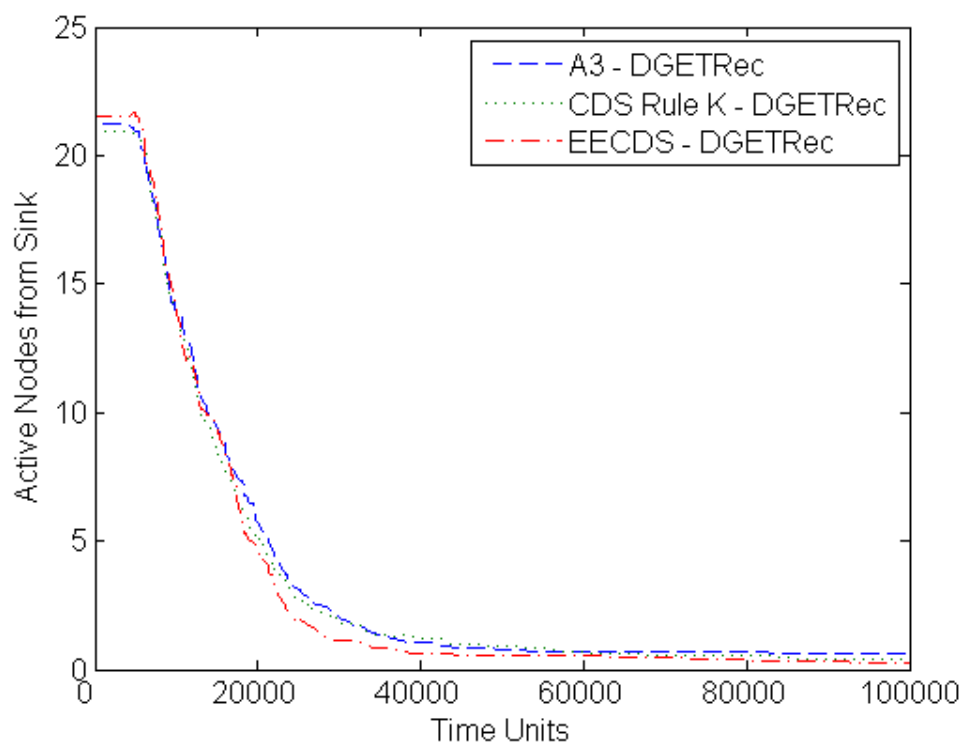

Figure 6.7: Best performing dynamic global topology maintenance techniques in sparse networks.

based techniques; it just means that they provided a worse performance with those particular settings, i.e. 1000 time units for the time-based criterion, and $10 \%$ of the total remaining energy for the energy-based criterion. More insights about this aspect are included in Section 6.8.

Figure 6.6.2.2 plots the best performing mechanisms in each case. As in previous cases, it seems that the topology maintenance mechanism based on the A3 topology construction algorithm has a better performance than the ones based on the CDS-Rule-K and EECDS algorithms, however, the advantage is marginal. Although A3 has better message complexity than CDS-Rule-K and EECDS, the small amount of sensors (100) and the small energy threshold (10\%) cause the topology maintenance algorithm to call the topology construction algorithm very few times, which makes the amount of energy spent by the three schemes very similar. A more pronoundced lifetime advantage of A3 would had been seen if the topology construction algorithm had been run many more time. 


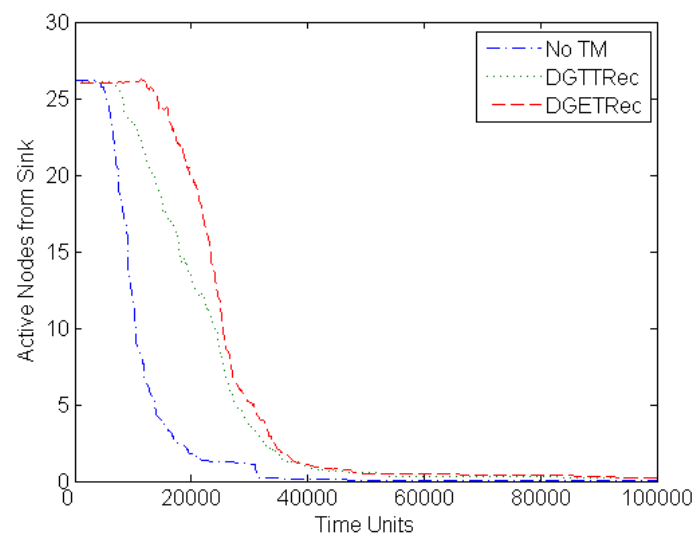

(a) A3

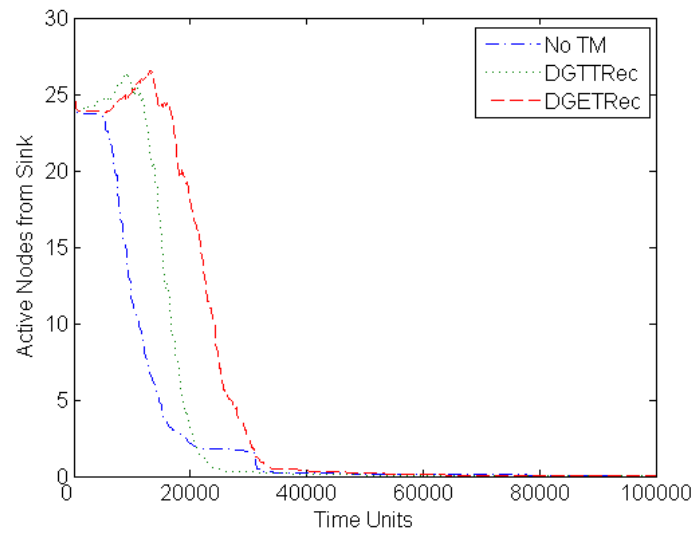

(b) EECDS.

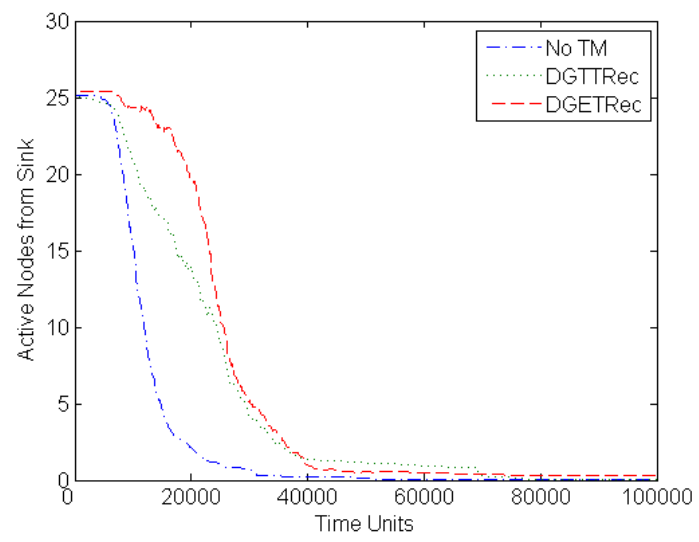

(c) CDS-Rule-K

Figure 6.8: Network lifetime with and without dynamic global topology maintenance using the A3, EECDS, and CDS-Rule-K topology construction mechanisms in dense networks. 


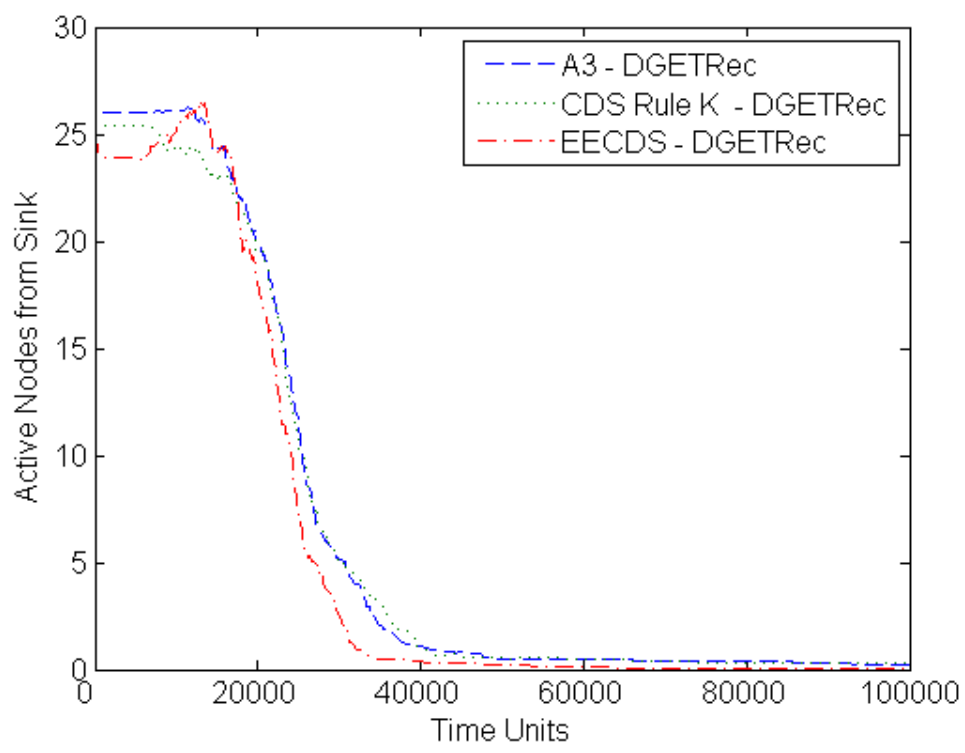

Figure 6.9: Best performing dynamic global topology maintenance techniques in dense networks.

\subsubsection{Performance Evaluation of Dynamic Local Topology Maintenance Tech- niques}

The dynamic local topology maintenance technique was also implemented in the Atarraya simulator and evaluated using the same parameters and scenarios utilized before. In the next subsections the results in sparse and dense networks are presented where the following topology maintenance techniques and triggering criteria are included:

- No Topology Maintenance - No TM: The initial reduced topology works permanently until the sink detects that it does not have any more active nodes in range. This is the same termination policy for the rest of the algorithms.

- Dynamic Local Energy-and DSR-based Repair - DLEDSR: Every time a node reaches a critical energy threshold, the local topology maintenance algorithm based on the DSR protocol repairs the topology. 


\subsubsection{Sparse Networks}

Figure 6.10 shows the simulation results of the dynamic local topology maintenance technique compared with the case of no topology maintenance in sparse networks using the A3, EECDS, and CDS-Rule-K topology construction algorithms. As it is clearly shown, the dynamic local technique enhances the network lifetime in all cases, meaning that, even in sparse networks, it is worth applying. Another observation is that in this case, the technique based on the CDS-Rule-K topology construction mechanism is the best performing one (See Figure 6.6.3.1). This is because the CDS-Rule-K algorithm somehow builds trees with close branches, and the local procedure is able to restore the connectivity of the branch with very few nodes, as compared with A3 and EECDS. This effect is better seen after the topology has been run for some time, after 15000 time units in the figures. At that time, many nodes start reaching the energy threshold, triggering the local procedure more and more often. As time continues, the number of nodes triggering the local procedure increases making the advantage of CDS-Rule-K more appreciable over the A3 and EECDS counterparts.

\subsubsection{Dense Networks}

The simulation results of the energy- and DSR-based dynamic local technique in dense networks are presented in Figure 6.12. As before, the results improve with the density of the network, as more possibilities exist to reconnect the topology when more nodes are available. As the figure shows, in all cases the application of the dynamic local topology maintenance technique improves the network lifetime compared with the case of no topology maintenance. Further, the performance also improves over the case with sparse 


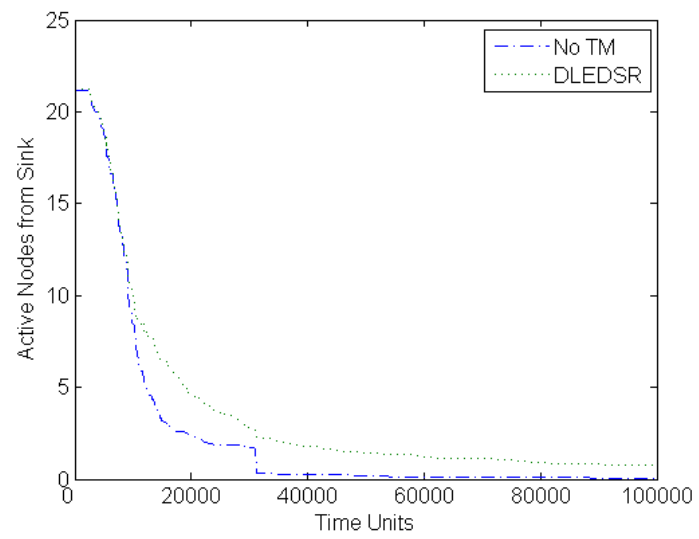

(a) $\mathrm{A} 3$

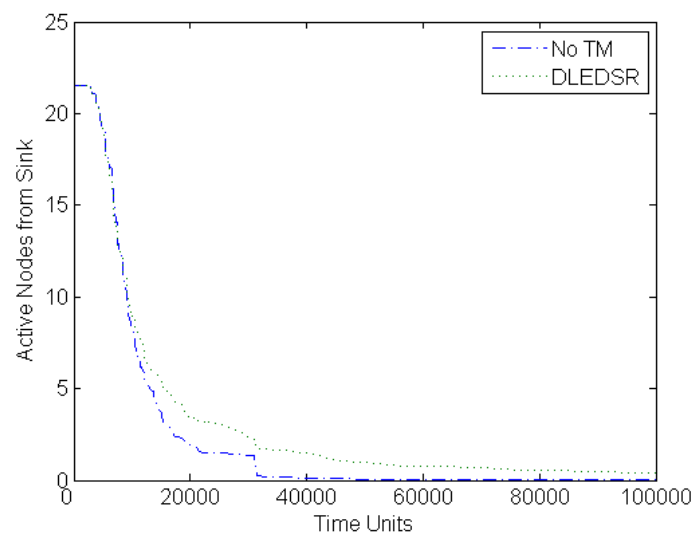

(b) EECDS.

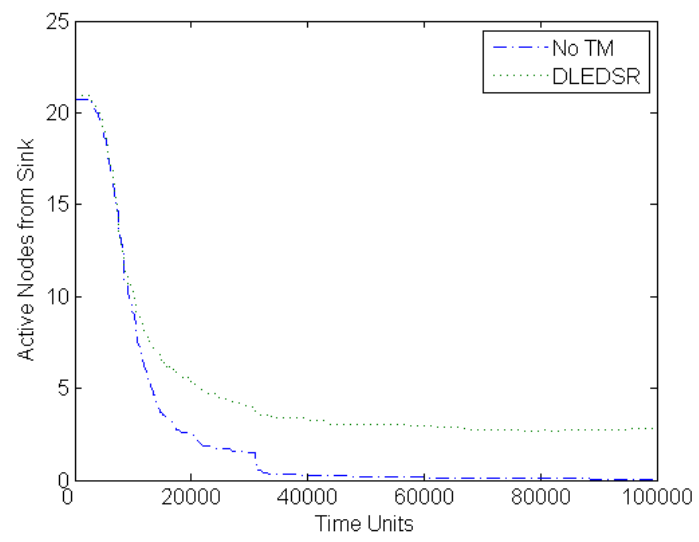

(c) CDS-Rule-K

Figure 6.10: Network lifetime with and without dynamic local topology maintenance using the A3, EECDS, and CDS-Rule-K topology construction mechanisms in sparse networks. 


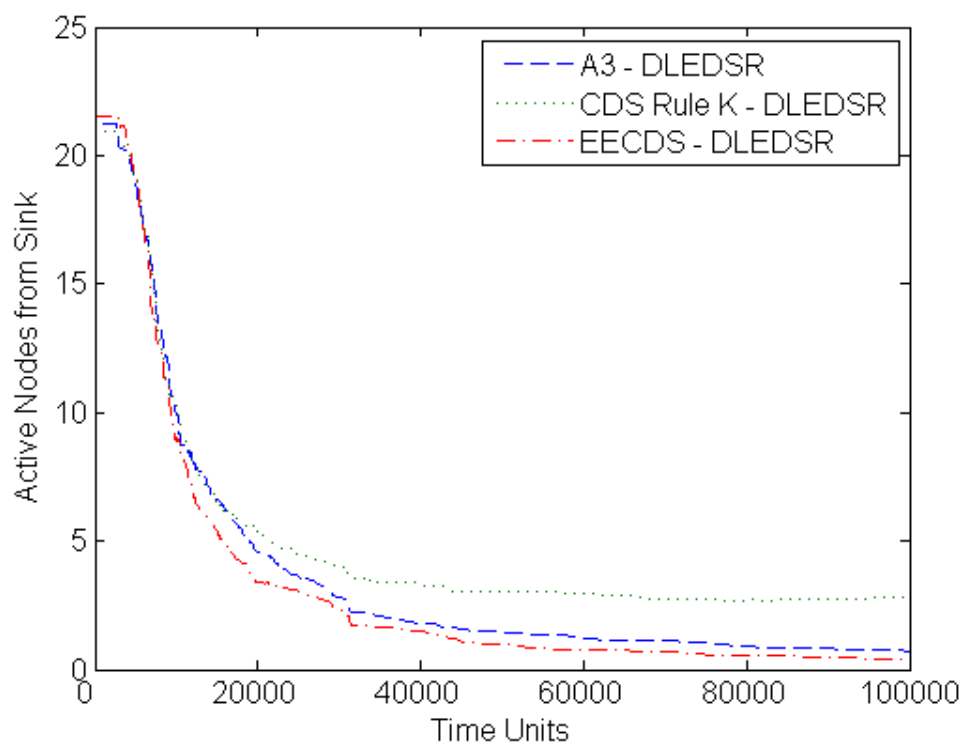

Figure 6.11: Best performing dynamic local topology maintenance techniques in sparse networks.

networks, as expected. Similar to the results in sparse networks, Figure 6.6.3.2 shows that the local technique based on the CDS-Rule-K algorithm outperforms the other two. The behavior is the same only that with more nodes the performance is better. The CDS-RuleK topology construction algorithm fits very well into this dynamic local strategy.

\subsubsection{Performance Evaluation of Hybrid Global Topology Maintenance Techniques}

Using the same network parameters described in Table 6.1, and the same underlying topology construction mechanisms utilized in the performance evaluations included in sections 6.2 and 6.3, simulation experiments were carried out to assess the network lifetime of the hybrid global topology maintenance technique just described in sparse and dense networks. The following topology maintenance techniques and triggering criteria were included: 


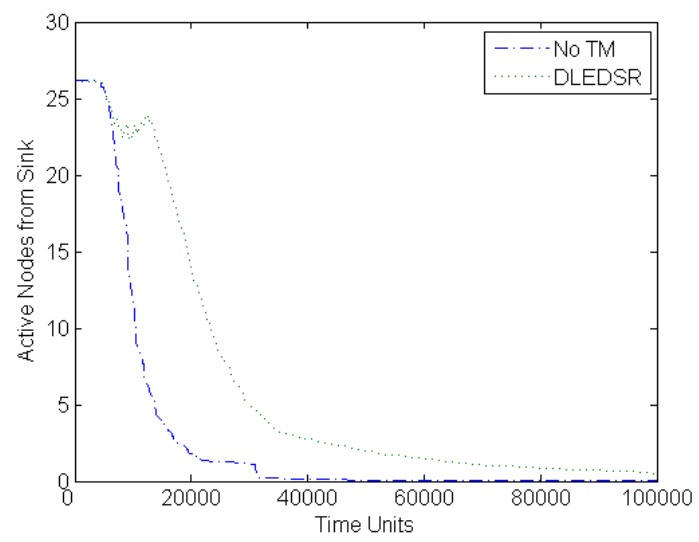

(a) $\mathrm{A} 3$

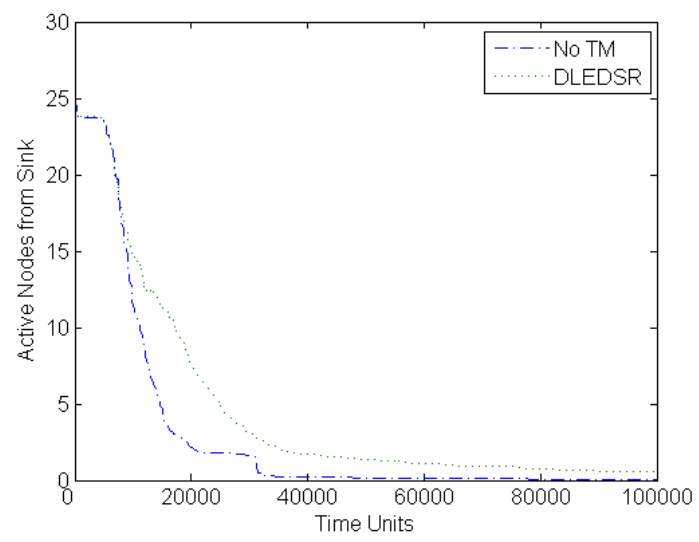

(b) EECDS.

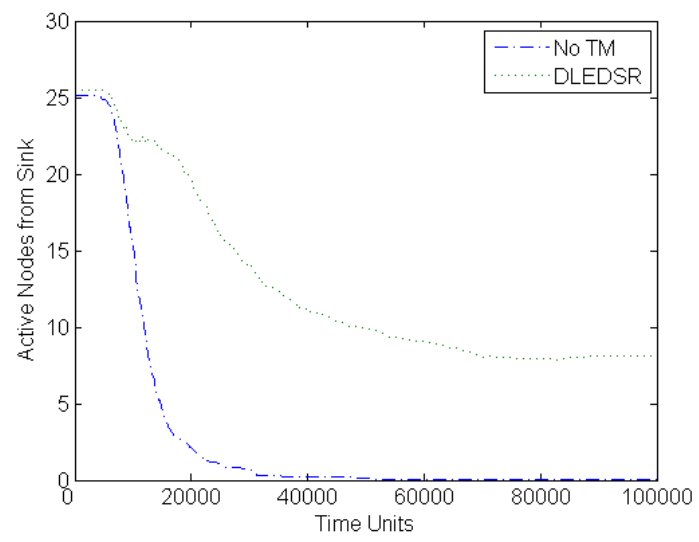

(c) CDS-Rule-K

Figure 6.12: Network lifetime with and without dynamic local topology maintenance using the A3, EECDS, and CDS-Rule-K topology construction mechanisms in dense networks. 


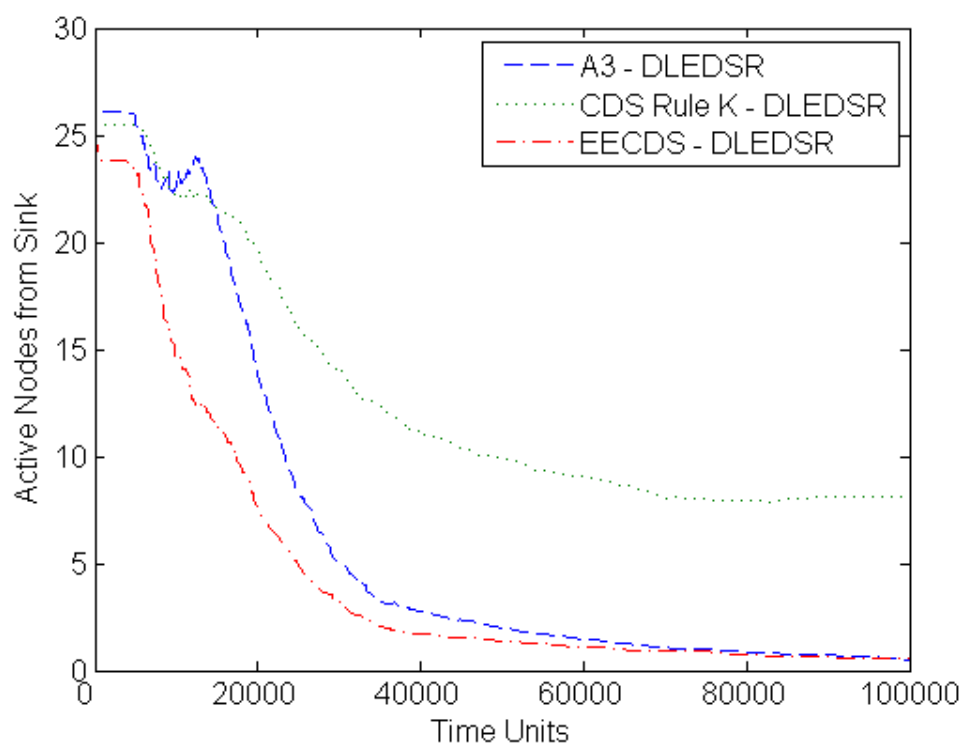

Figure 6.13: Best performing dynamic local topology maintenance techniques in dense networks.

- No Topology Maintenance - No TM: The initial reduced topology works permanently until the sink detects that it does not have any more active nodes in range. This is the same termination policy for the rest of the algorithms.

- Hybrid Global Time-based Topology Recreation Rotation - DGTTRecRot: Every set time interval the topology maintenance algorithm rotates the active reduced topology for one of the preplanned ones. If the new pre-planned topology cannot provide the expected service (has no connection with the sink), the hybrid topology maintenance algorithm invokes the topology construction algorithm to create a new reduced topology on the fly.

- Hybrid Global Energy-based Topology Recreation Rotation - DGETRecRot: Every time a node reaches a critical energy threshold, the topology maintenance algorithm rotates the active reduced topology for one of the preplanned ones. If the new preplanned topology cannot provide the expected service (has no connection with the 
sink), the hybrid topology maintenance algorithm invokes the topology construction algorithm to create a new reduced topology on the fly.

\subsubsection{Sparse Networks}

As in the case of the static and dynamic topology maintenance techniques presented in sections 6.2 and 6.3, the performance results of the hybrid global technique presented here shows no major improvement compared with the no topology maintenance option in sparse networks. In some cases, the technique increases the network lifetime and in some cases reduces it, but with small margins in both cases, emphasizing the conclusion reached before with other techniques that applying topology maintenance in sparse networks will not produce significant improvements in terms of network lifetime.

Figure 6.6.4.1 also emphasizes previous results. For example, it shows that the energybased hybrid technique also outperforms the time-based technique. Also, the performance

of the hybrid technique based on the A3 topology construction is consistent with previous results, showing its advantage although without impressive margins. Finally, Compared with the static and dynamic counterparts, it seems that the hybrid technique improves the performance, but not considerably. (More on this in Section 6.7 later.)

\subsubsection{Dense Networks}

The same experiments were run in a more dense network, one with 100 nodes -twice the number of nodes utilized in the sparse scenario. The simulation results shown in Figure 6.16 clearly demonstrate the power of this technique. In all cases the hybrid global topology maintenance technique outperforms the no topology maintenance option by impressive margins. As in previous cases, Figure 6.6.4.1 shows that the hybrid technique 


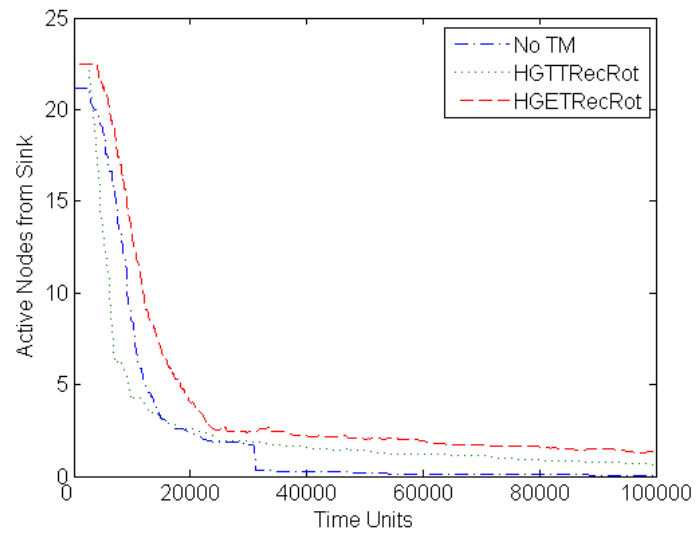

(a) A3

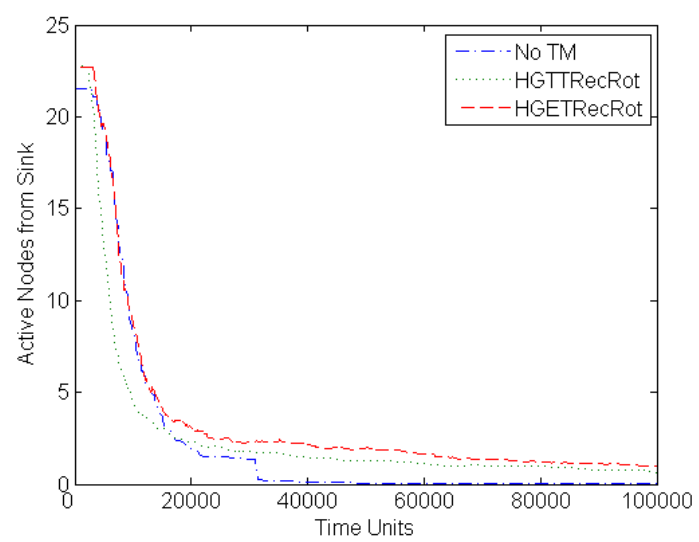

(b) EECDS.

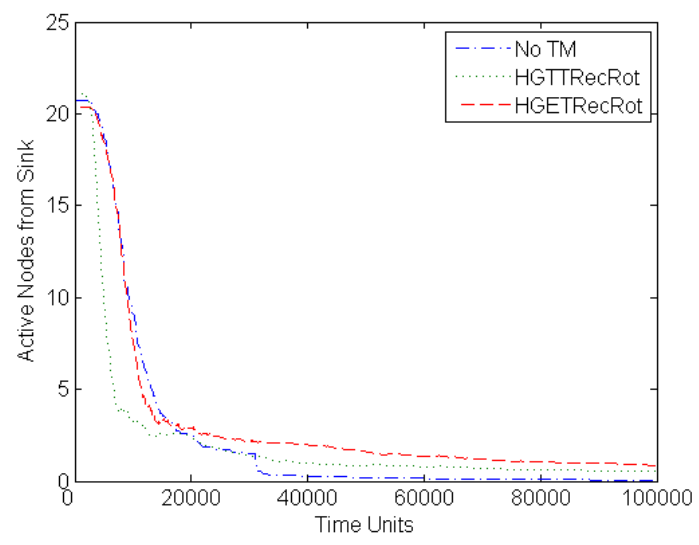

(c) CDS-Rule-K

Figure 6.14: Network lifetime with and without hybrid global topology maintenance techniques using the A3, EECDS, and CDS-Rule-K topology construction mechanisms in sparse networks. 


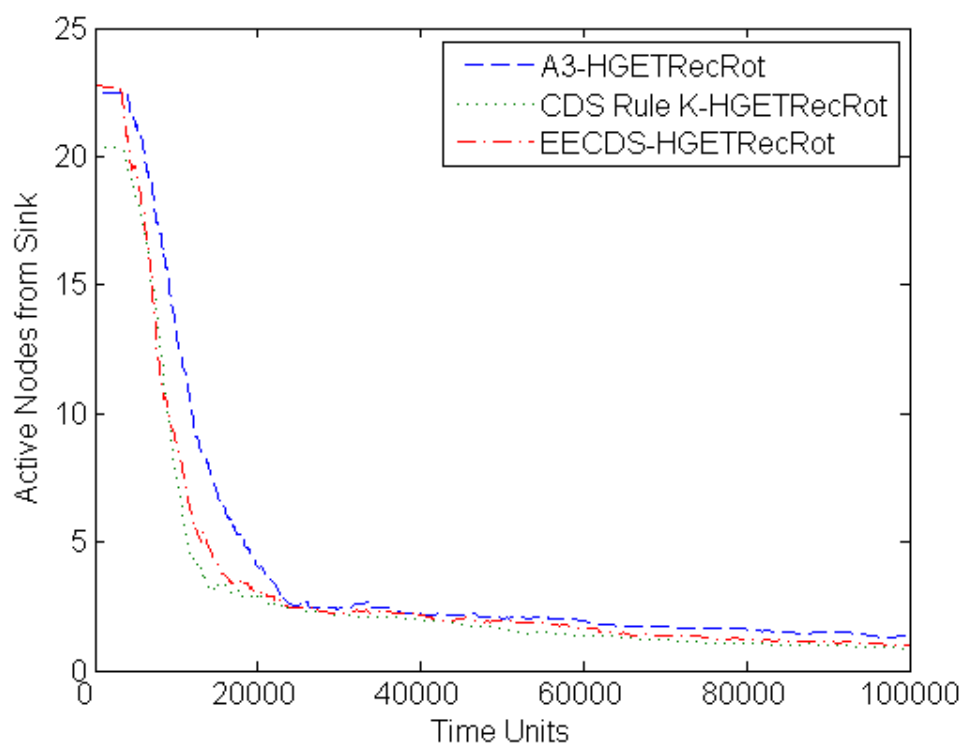

Figure 6.15: Best performing hybrid global topology maintenance techniques in sparse networks.

with the A3 topology construction algorithm performs better than the technique with the EECDS and CDS-Rule-K algorithms. It is clear that, once the static portion of the technique exhausts the energy of the pre-planned trees, the dynamic technique still can find new trees, and therefore, run the network longer.

\subsection{Comparison of Topology Maintenance Techniques}

This section summarizes all the performance evaluation results presented thus far in this part of the book in order to provide general conclusions as to which technique is better to use in sparse and dense networks. As such, this section is meant to answer questions, such as:

- What is the best static, dynamic and hybrid technique in sparse and dense networks?

- If I have a sparse or a dense network, what technique should I use? 


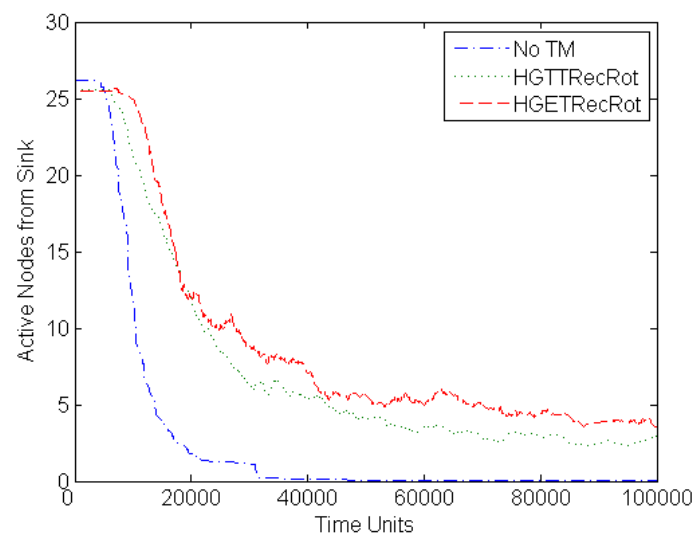

(a) $\mathrm{A} 3$

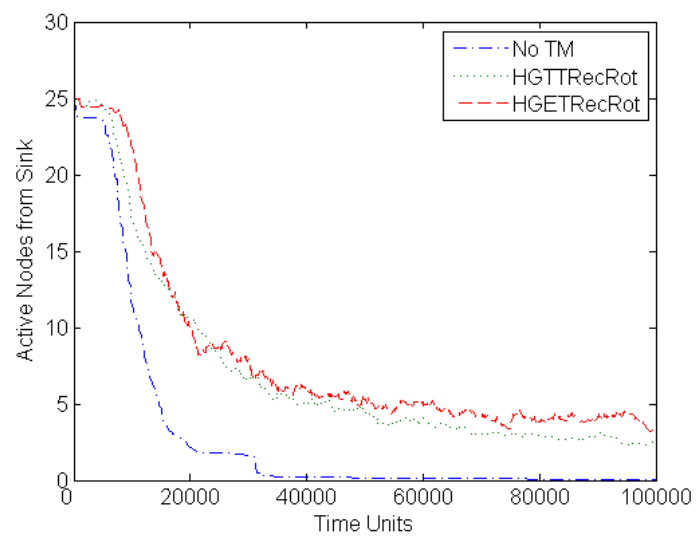

(b) EECDS.

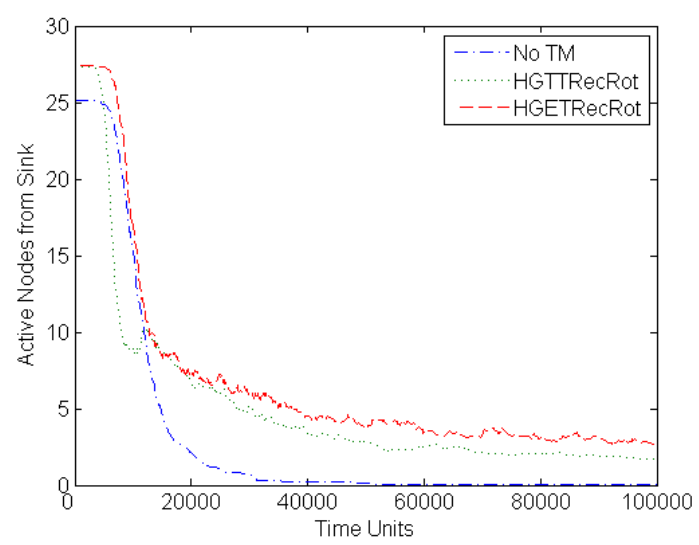

(c) CDS-Rule-K

Figure 6.16: Network lifetime with and without hybrid global topology maintenance techniques using the A3, EECDS, and CDS-Rule-K topology construction mechanisms in dense networks. 


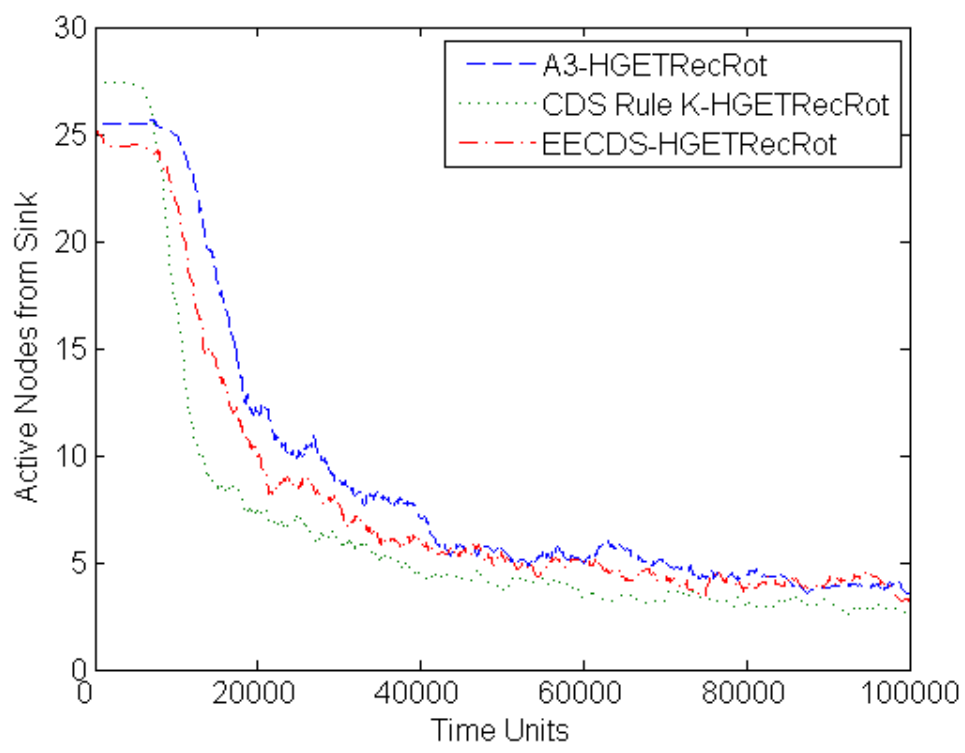

Figure 6.17: Best performing hybrid global topology maintenance techniques in dense networks.

- Which approach is better, global or local?

- What triggering criteria is better, energy-based or time-based?

All these questions can be answered by looking at the results presented in Figure 6.18, which includes the best performing techniques in sparse and dense networks using the same simulation parameters and scenarios outlined thus far. From the figure, three general conclusions can be easily drawn. First, consistent with all results presented so far, it can be seen that regardless of the network density, the scope of the technique (global or local), and type of technique (static, dynamic or hybrid) the energy-based techniques are the best performing ones. Second, the global techniques (either static, dynamic, or hybrid) perform better with the A3 topology construction algorithm. Finally, the local DSR-based technique based on the CDS-Rule-K topology construction algorithm outperforms the global techniques in both sparse and dense networks. Therefore, the energy-, DSR- and 


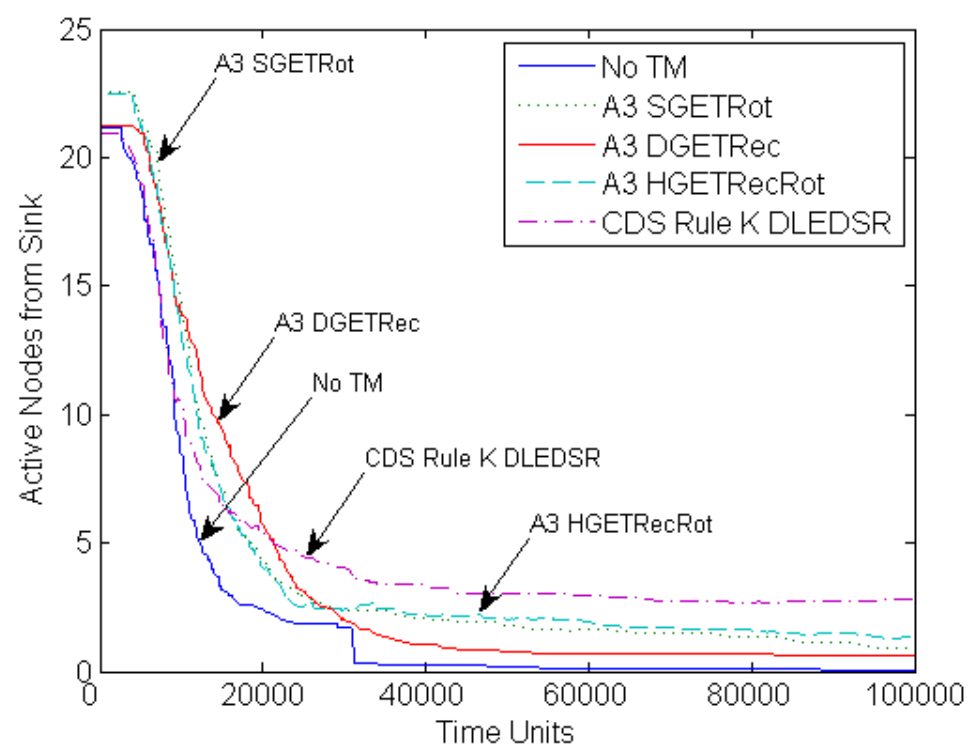

(a) Best of all in sparse networks.

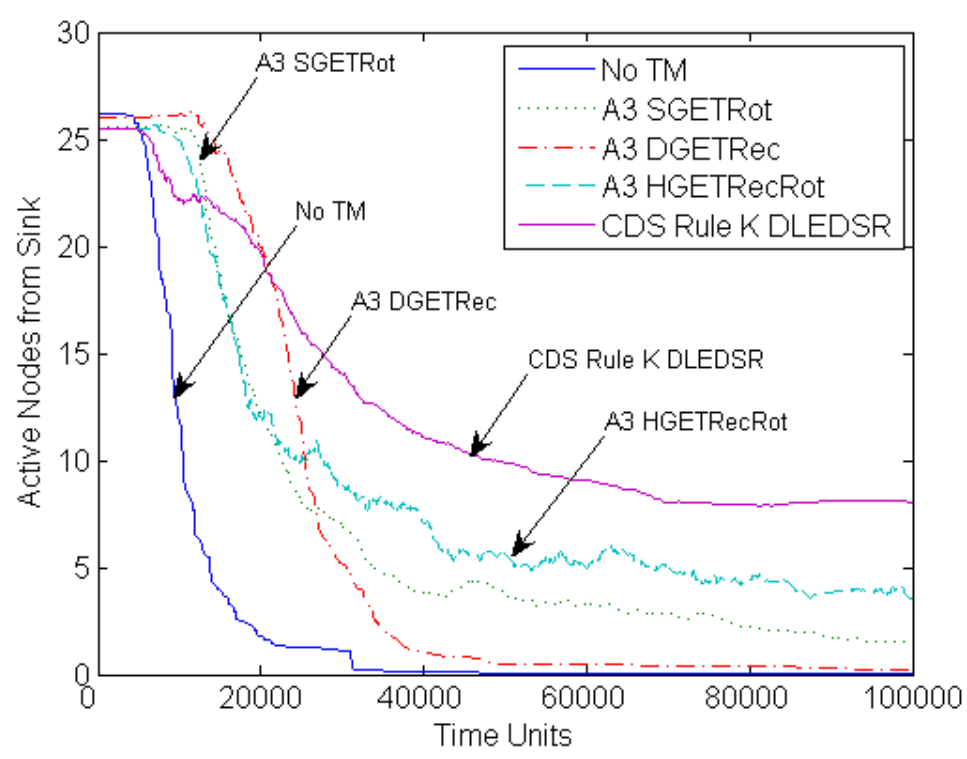

(b) Best of all in dense networks.

Figure 6.18: Best performing topology maintenance techniques in sparse and dense networks. 
CDS-Rule-K-based dynamic local topology maintenance technique is the best performing technique in sparse and dense networks.

Another important conclusion about the behavior of these techniques is that dynamic global techniques tend to offer better performance longer than static and hybrid global techniques, but once the performance starts to decay, it decreases faster. The performance of static global techniques starts to decay sooner but the decrease is not as sharp, being able to live longer than dynamic global techniques over longer periods of time. The hybrid global techniques are a good compromise. They have the same performance as static global techniques during the initial continuous time but their performance over time is considerably better than static or dynamic global techniques, with a smoother network lifetime decay. Finally, the DSR-and CDS-Rule-K-based dynamic local technique presents the worst performance at the beginning, meaning that nodes start to die faster, but shows the best performance of all over time.

More specific conclusions can be drawn looking at each figure individually. For example, from Figure 6.18(a), it can be seen that, again, topology maintenance techniques in sparse networks do not improve the lifetime of the network by an important margin compared with the no topology maintenance option. This is definitively not the case in dense networks, as it can be seen from Figure 6.18(b), where the positive effect of the topology maintenance techniques over the network lifetime is appreciable.

In conclusion, dynamic global techniques are the best option if a continuous operation of the entire network is needed over a specific amount of time, less than the time at which they start to die fast. If the application, on the other hand, requires the network to be alive longer even with fewer nodes and maybe with some areas not completely covered, then the dynamic local technique using the CDS-Rule-K topology construction mechanism is the best way to go. A good compromise in this spectrum of possibilities is the global 
hybrid technique, which provides better performance than the local technique at the beginning (works with all the nodes longer), and an intermediate performance over time compared with the dynamic global and the local techniques.

It is important to mention that these general and specific conclusions only represent one picture of the entire spectrum of possible scenarios. All these conclusions have been drawn from the simulation results of experiments run with 50 and 100 nodes, an energy threshold of $10 \%$, a time threshold of 1000 time units, maximum of 3 reduced topologies in the case of static techniques, the A3, CDS-Rule-K, and EECDS topology construction algorithms, and the local DSR-based topology maintenance mechanism. In the next section, these results are extended by running additional experiments varying the energy and time thresholds, and the network density.

\subsection{Sensitivity Analysis}

Thus far, all simulation experiments have been performed using 50 or 100 nodes to represent sparse and dense network scenarios, respectively, and fixed energy and time thresholds, of $10 \%$ and 1000 time units, as defined in Table 6.1. This section is meant to provide more insights about the performance of the topology maintenance techniques under consideration when these parameters are varied. In what follows, simulation results are presented when the time threshold is varied from 600 to 1000 to 5000 to 10000 units; the energy threshold is changed from $5 \%$ to $10 \%$ to $25 \%$ to $50 \%$ of the remaining energy in the node; and the number of nodes is varied from 50 to 100 to 400 nodes. Experiments are run using static, dynamic, and hybrid global techniques using the A3 algorithm as the only topology construction algorithm, and the DSR-based dynamic local technique using the CDS-Rule-K algorithm only. Also, all experiments are run in dense networks only. 
These decisions are based on the superior performance of the global techniques based on the A3 algorithm, the superiority of the local technique based on the CDS-Rule-K algorithm, and the fact that, in most cases, topology maintenance is worth applying in dense networks only.

\subsubsection{Time-based Analysis}

Figure 6.19 shows the network lifetime results for the static, dynamic, and hybrid global topology maintenance techniques with time thresholds of 600, 1000, 5000, and 10000 time units using the A3 topology construction algorithm. The figure provides insights on two fronts. First, it immediately tells about the impact of changing the time threshold in the network lifetime. As it can be observed, in all cases, the network lifetime seems to be mostly unaffected by the time threshold. However, the figures clearly show the effect of the threshold in the operation of the network. For example, the figures show the bumpy behavior of the techniques when the threshold is set at 10000 units. It is clear that the threshold time is big enough as to allow many nodes to die before it expires, and when it expires, the new topology is able to include more active nodes than the topology just replaced. On the other hand, this effect is not seen when a small threshold is used, such as in the case of the 600 time units threshold.

Second, the figure provides an overall comparison between static, dynamic, and hybrid global techniques. As it can be observed from Figure 6.20, a compromise between performance and smooth behavior is achieved when the time threshold is set to 5000 time units. As it can be seen, the best performing technique in each case is the one using this threshold of 5000 time units. At the same time, although the dynamic technique still presents some bumps, it is considerably better than the case with 10000 units. Of the three 


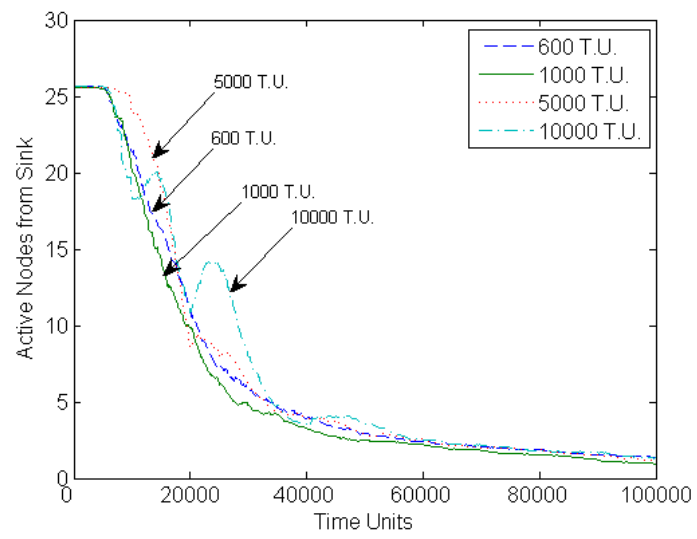

(a) Static technique.

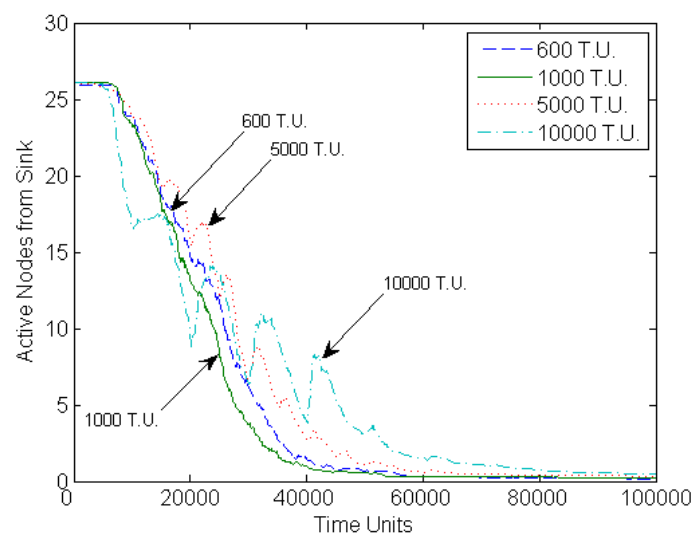

(b) Dynamic technique.

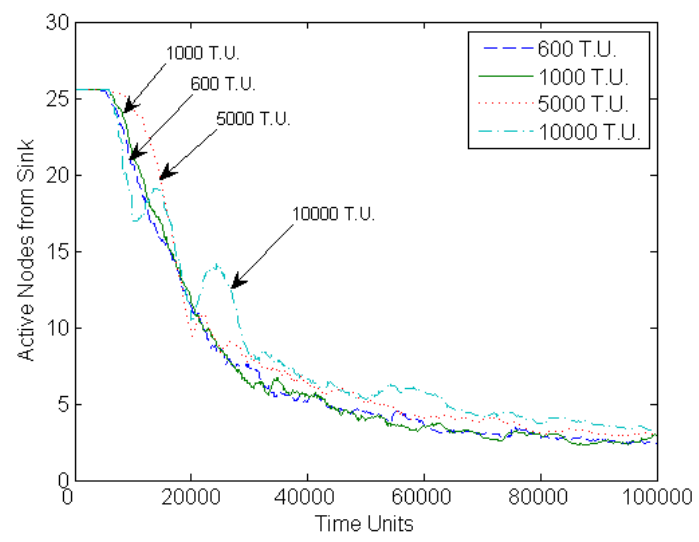

(c) Hybrid technique.

Figure 6.19: Time-based sensitivity analysis of network lifetime for static, dynamic, and hybrid topology maintenance techniques. 


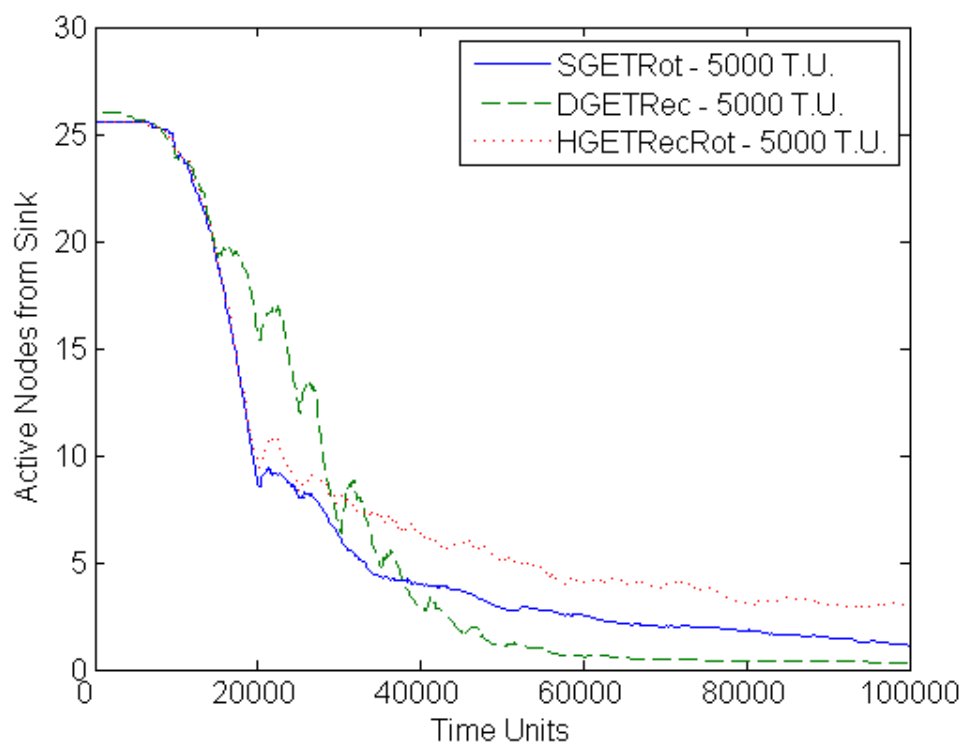

Figure 6.20: Best performing techniques out of time sensitivity tests.

best techniques, the figure also shows that the hybrid technique is the most appropriate, presenting the same performance of the other techniques during the initial 15000 time units but decaying slower as the time increases.

In conclusion, it can be said that, as expected, increasing the time provides better performance, as the topology construction mechanism needs to be run fewer times, but increasing the time threshold too much also produces bumpy behavior. Therefore, a good compromise is to set the threshold to a value close to the time at which the nodes are expected to start dying.

\subsubsection{Energy-based Analysis}

In this part similar simulation results are provided by changing the energy threshold. Figure 6.21 shows the network lifetime results for the static, dynamic, and hybrid global topology maintenance techniques, and the DSR-based dynamic local topology mainte- 


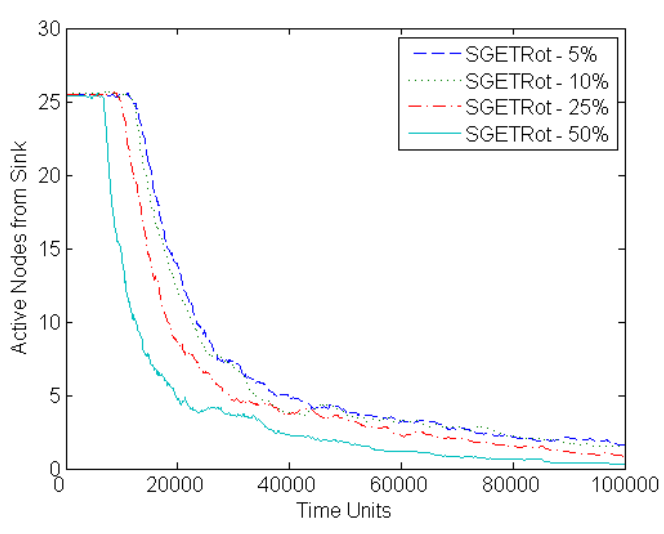

(a) Static technique.

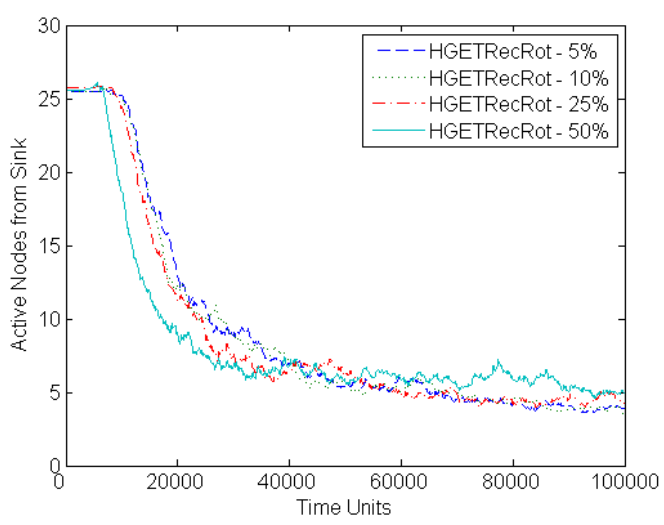

(c) Hybrid technique.

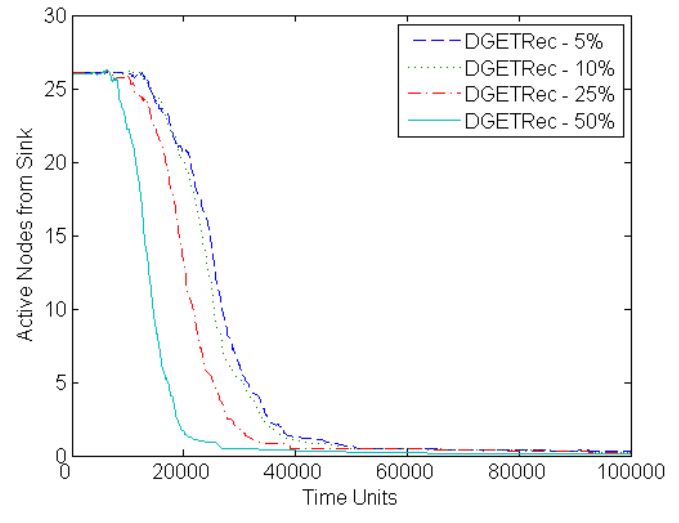

(b) Dynamic technique.

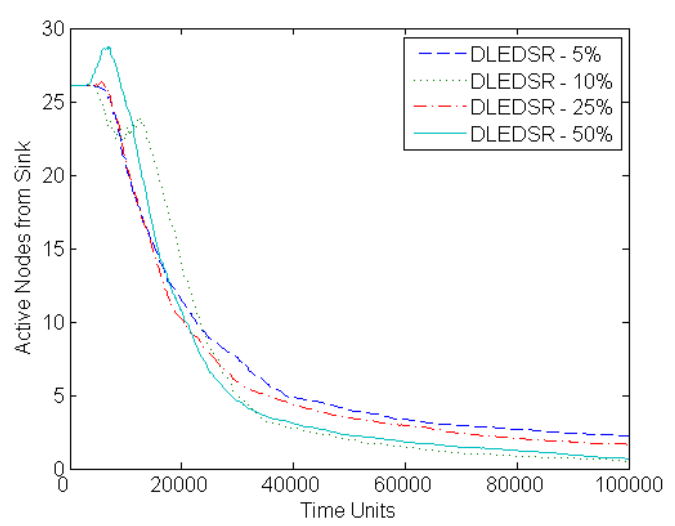

(d) DSR-based dynamic local technique.

Figure 6.21: Energy-based sensitivity analysis of network lifetime for static, dynamic, and hybrid topology maintenance techniques.

nance technique with energy thresholds of $5 \%, 10 \%, 25 \%$, and $50 \%$ of the node's remaining energy using the A3 topology construction algorithm. The figure immediately provides insights about the impact of changing the energy threshold in the network lifetime. As it can be observed, there is a general trend: the network lifetime improves with the reduction of the energy threshold. A smaller threshold corresponds to fewer runs of the topology construction mechanism, saving more energy.

Figure 6.22 provides an overall comparison between the techniques. The figure clearly shows that in this case, the hybrid technique is the best of all, starting its performance 


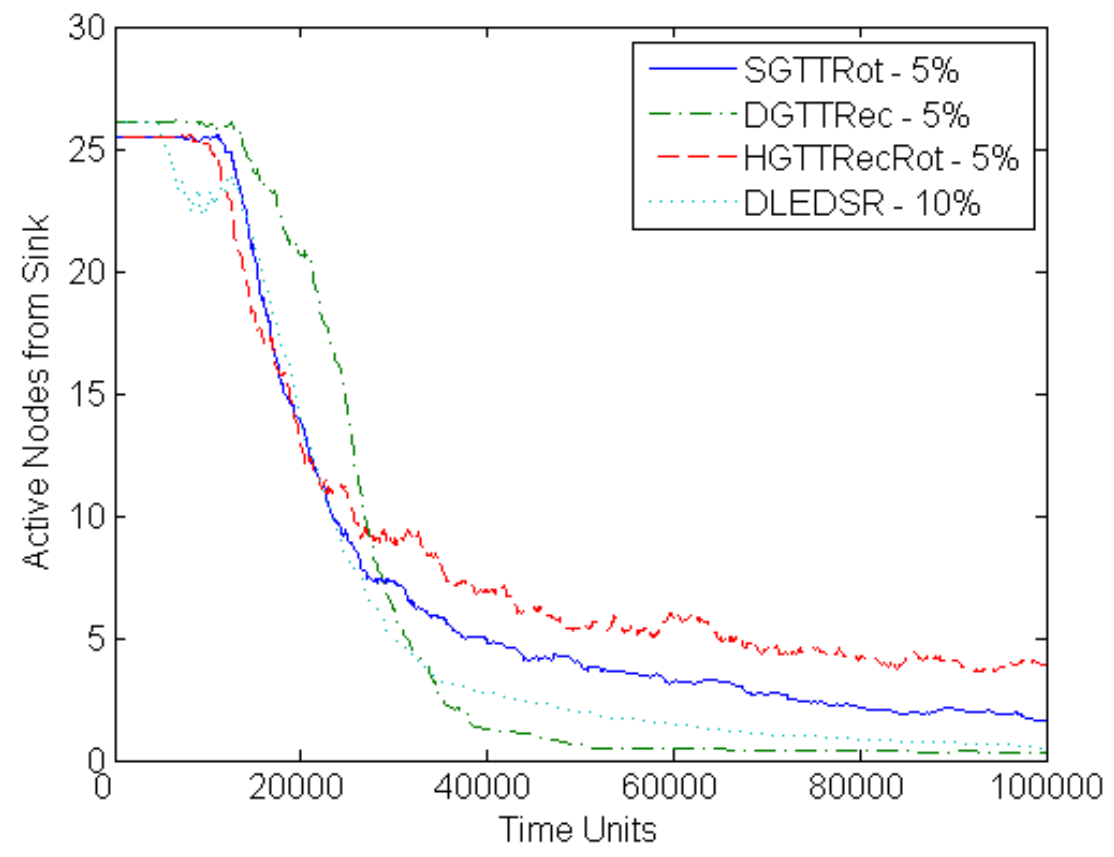

Figure 6.22: Best performing techniques out of energy sensitivity tests.

decay almost at the same time as the rest of the policies, but outperforming them all over time. Given the results obtained in these last two sections, it can be concluded that hybrid techniques are the best overall, as they get the best of the static and dynamic techniques they are made of. It is important to mention that the local technique does not perform as well as the global ones because it is based on the A3 topology construction algorithm. As shown in Figure 6.12(c), the inclusion here the performance of the local technique with the CDS-Rule-K algorithm would have shown that it is the best performing technique.

\subsubsection{Density-based Analysis}

Finally, experiments were also carried out to assess the performance of the static, dynamic, and hybrid global techniques, and the dynamic DSR-based local technique in scenarios with different node densities using the A3 topology construction algorithm. 


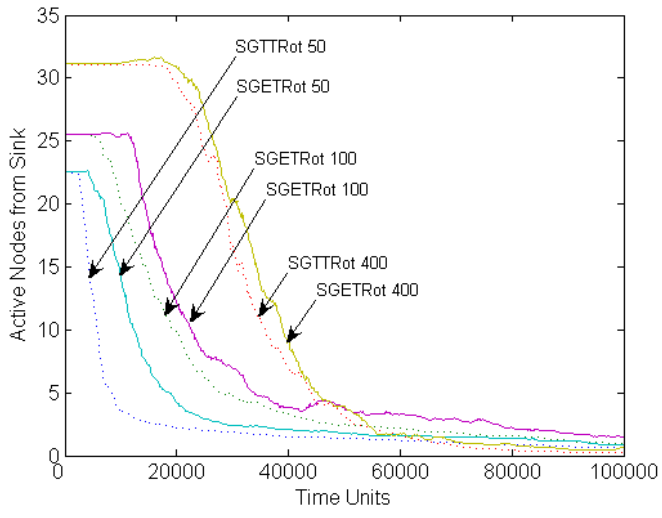

(a) Static technique.

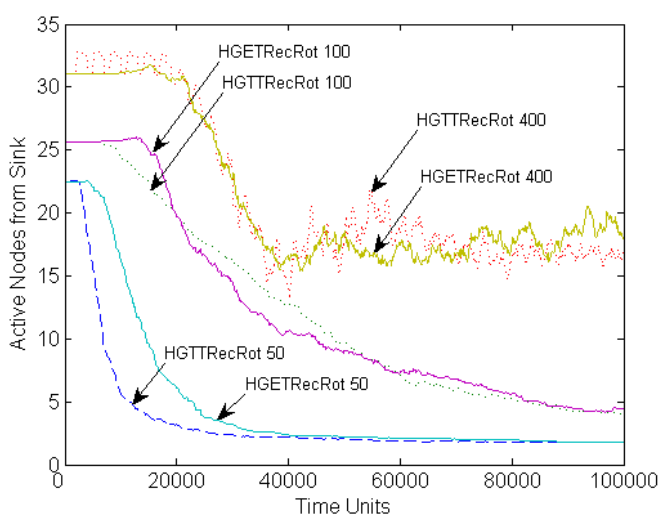

(c) Hybrid technique.

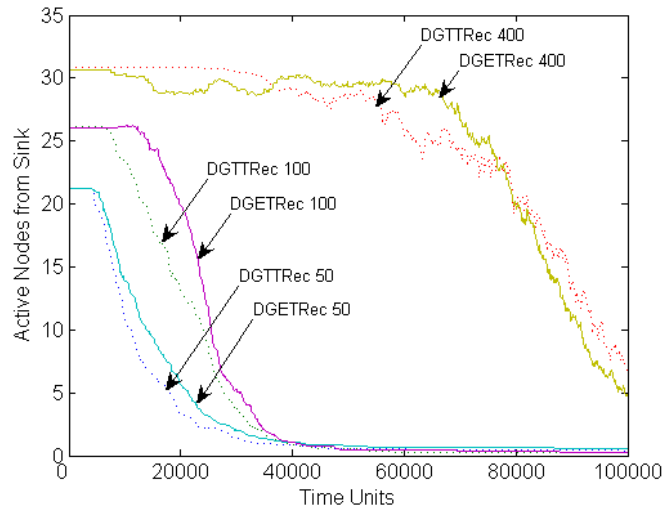

(b) Dynamic technique.

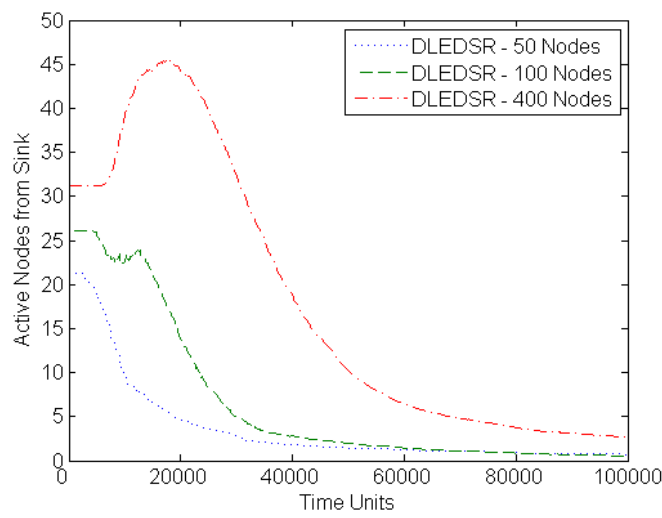

(d) DSR-based dynamic local technique.

Figure 6.23: Node density-based sensitivity analysis of network lifetime for static, dynamic, and hybrid topology maintenance techniques. 
Figure 6.23 shows the network lifetime results of the techniques under consideration with 50, 100, and 400 nodes using an energy threshold of $10 \%$ and a time threshold of 1000 time units. A general, and expected, trend can be easily observed from the figure: the network lifetime increases with the network density. As more nodes are added into the network, more disjoint topologies can be formed, and therefore the total lifetime can be increased. Also, more nodes increase the network wide energy resources. Another observation consistent with past results, is that the energy-based techniques outperform their time-based counterparts. Finally, Figure 6.21(d) shows the performance of the DSRbased energy-based dynamic local technique, which shows mixed results compared with the global techniqes, but definitively, it shows its value in some instances. Recal that this local technique utilizes the A3 algorithm, and better performance results are obtained with the CDS-Rule-K algorithm.

An interesting result is shown in Figure 6.23(c) where the effect of combining static and dynamic techniques can be clearly seen. At the beginning and during the first 20000 time units, the hybrid technique operates as the static one, as all topologies included in the rotation list can actually communicate with the sink node, with all their nodes participating in the topology. However, after 20000 time units, nodes start to die and the performance of the hybrid technique starts to decay following the slope of the static technique, as up to this point, the dynamic part of the technique has not been activated for the first time. It is at 40000 time units when the dynamic techniques start working, as some topologies in the static rotation list have no communication with the sink node any more. From this time until approximately 150000 time units (not shown in the figure) the performance of the hybrid technique is fairly stable. After that, most of the nodes die and the dynamic technique can no longer find more topologies. 


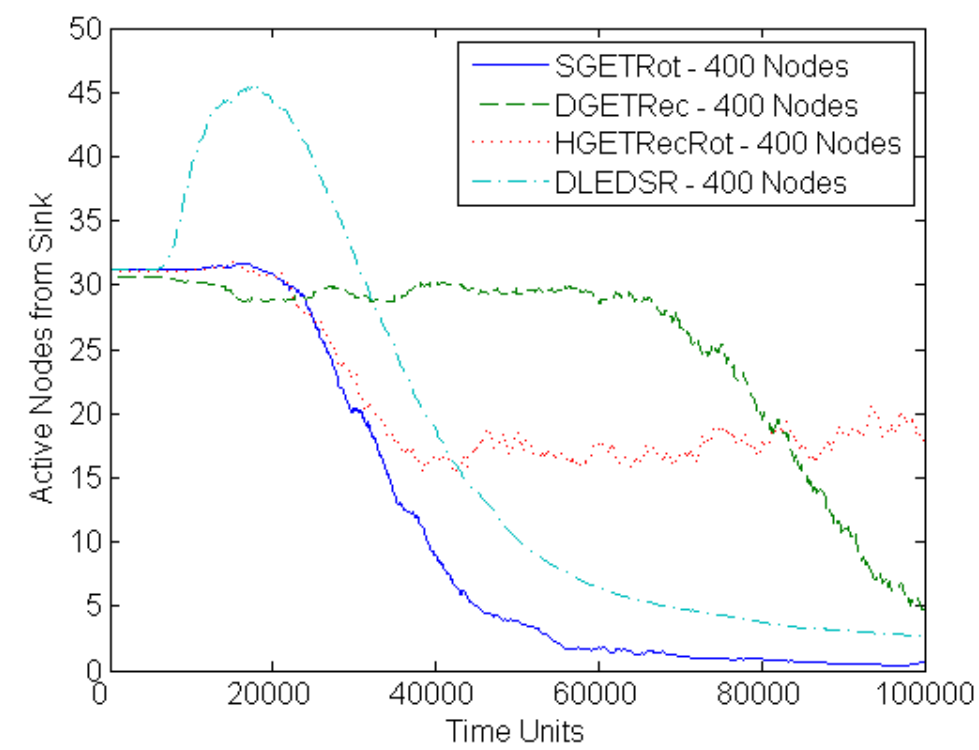

Figure 6.24: Best performing techniques out of the density sensitivity tests.

Comparing these results, it can be concluded that increasing the network density can increase the network lifetime considerably. Also, among these techniques, the dynamic energy-based technique offers the best performance overall during a fairly large amount of time, after which the hybrid technique takes over maintaining an still very acceptable performance for almost twice the original time. Figure 6.24 includes the best performing techniques in each case, where this last conclusion is easily observed.

Finally, one last experiment was performed in order to present the total benefits that applying a topology control mechanism brings to the lifetime of the network. Three cases where considered: when no topology control is applied (no topology construction or maintenance), when just topology construction is applied with no maintenance policy, and when a complete topology control scheme is applied, including topology construction and maintenance. Figure 6.25 shows the comparison of the network lifetime, using the A3 topology construction protocol and the DGETRec topology maintenance protocols, in scenarios with 400 nodes. The lifetime comparison took place in two moments: first, 


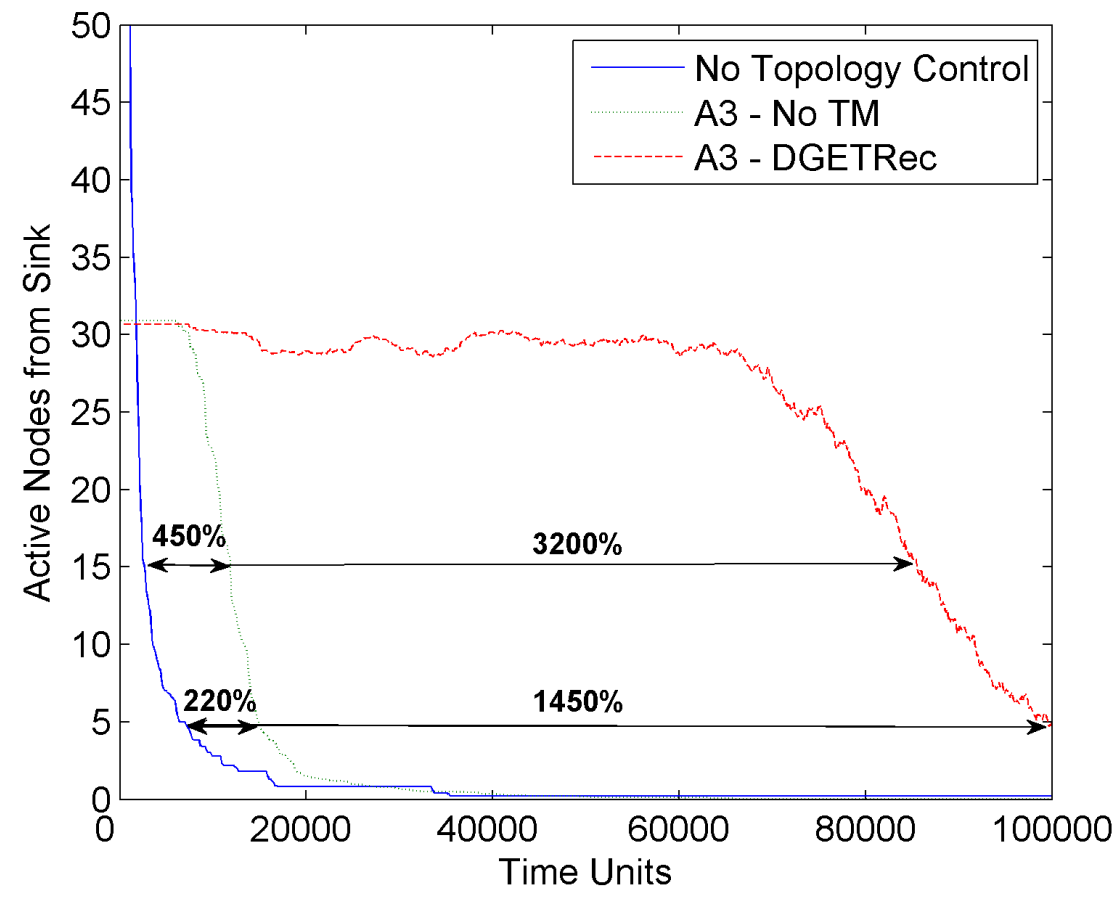

Figure 6.25: Comparison of network lifetime when using topology construction and maintenance, topology construction only and no topology control.

when the number of active nodes of the reduced topologies reaches half the initial size (15 nodes), and 5 active nodes when the network is almost dead. In the first case, when the number of active nodes is 15 , the results shows how the lifetime of the network is extended in $3200 \%$ when using both topology construction and maintenance, and $450 \%$ when using only a topology construction mechanism. In the second case, when the number of active nodes is 5 and the network is almost dead, the results shows a gain of $1450 \%$ when using both topology construction and maintenance, and $220 \%$ when using only a topology construction mechanism. These gains show the power of topology control techniques in wireless sensor networks as a tool for extending the lifetime of the network. 


\section{Chapter 7: Conclusions and Future Work}

\subsection{Conclusions}

Topology control is an area that has seen an increase in interest during the past decade, fact that is unmistakenly shown by the amount of published papers and books in the area. The nature of the solutions provided in this area started with theoretical and centralized approaches, which were based on traditional graph theory techniques, and then evolved into the present stage with a large selection of fully distributed and simple protocols that can run efficiently in constrained devices. In addition, the trend has grown from only offering connectivity in the network, into also guaranteeing coverage of the area of interest, with different levels of redundancy and, in many cases, without the need of localization information of the nodes in the network.

The A3 family of simple connectivity- and coverage-oriented protocols presented in this dissertation are clear examples of these solutions. The benefits of the application of these protocols was seen in the different performance evaluations. For example, the A3 and A3Lite protocols showed a considerable advantage in message and energy overhead compared to the other two distributed protocols EECDS and CDS-Rule-K, while producing very similar sized reduced topologies. In addition, the results of A3 and A3Lite were compared to the centralized MIP-MCDS formulation, and it could be seen that the average difference between the optimal results and the ones produced with the heuristics 
reached up to $37 \%$ in some scenarios. One interesting aspect of the results showed is that the heuristics were very close to the optimal solution in topologies with a small number of nodes, or when the node degree was very high. In the case of A3Cov and A3CovLite, they offered better coverage ratio than the ACOS protocol and similar coverage to the StanGA protocol; however, A3Cov and A3CovLite guarantee connectivity.

The benefits of topology control are very clear throughout this dissertation and all the referenced work in the literature. A wireless sensor network cannot afford not having some kind of topology control in order to guarantee the efficient use of its resources during the time of activity. This can be concluded from the performance evaluation of the different topology maintenance protocols. The difference, in terms of lifetime of the networks that did not have any maintenance protocols against the ones that did, shows the undeniable benefit of having a maintenance strategy in the network.

One topic that is expected to reach the interest of the research community is the separation of the concept of topology maintenance from the topology construction process, which simplifies the analysis, development and implementation of new topology maintenance protocols, and also their pairing with existing and new topology construction protocols in order to find a better performance. The importance of this fact can be seen in how the different maintenance approaches produced individual results not only based on the nature of the protocols but also based on the particular pairing of topology construction and maintenance that were evaluated.

The new concept of interdependence in topology control must also be acknowledge by simulation tools, which are always the first step in the implementation and testing process of new protocols. This characteristic is one of the main contributions that the new simulation tool Atarraya introduces to the market of simulators for topology control in wireless sensor networks. 


\subsection{Summary of Contributions}

This dissertation presents new contributions in the area of topology control for wireless sensor networks. This is a summary of the document and the contributions.

- A new definition of Topology Control is proposed, based on the separation of the TC process into two instances: topology construction and topology maintenance. Based on this definition, a new taxonomy is proposed in order to classify the existing protocols. A special distinction is made between connectivity- and coverageoriented topology construction protocols in the taxonomy, given the particularities of the protocols in each of these categories.

- A new mathematical formulation of the Minimal Connected Dominating Set, based on the Mixed Integer Programming approach, that solves in a parallel approach to the problems of dominance and connectivity using a modification of the multicommodity approach called "'flow of tokens"'. Some characteristics of this solution are that it uses a linear number of restrictions, that it runs in a single iteration and that it does not need preprocessing.

- Two new connectivity-oriented topology construction protocols are proposed, based on the hierarchical approach: A3 and A3Lite. These protocols "grow a tree" rooted at the sink node, create a CDS that connects all the nodes in the network, and then turns off all redundant nodes that do not offer connectivity to any of their neighbors. The simplicity of the protocols is one of the main reasons why these protocols excel in the performance evaluation compared to other well-known protocols of their same category, especially in terms of energy consumption and message overhead. 
- Two new coverage-oriented topology construction protocols are proposed, based on the A3 and A3Lite protocols: A3Cov and A3CovLite. These protocols start from the connected reduced topology produced by its predecessors, and then extend them by adding nodes that increase the area covered by the active network. In addition, they implement a simple policy that determines variable levels of coverage, as a trade off parameter between coverage and number of active nodes. The Cov protocols are compared with theoretical optimal solutions, and with two distributed protocols, in which the Cov protocols show superiority in coverage and connectivity.

- Four topology maintenance protocols are implemented: SGTRot, DGTRec, HGTRecRot and LD-DRS, with the last two being completely new protocols. Their performance is tested in order to evaluate the joint execution of topology construction and maintenance as complete integrated topology control solutions. The results show that in general the DGTRec protocol tends to provide a better performance, maintaining the highest possible number of active nodes until the resources are completely depleted, and that the HGTRecRot and LD-DSR produce good results depending on the topology construction protocol.

- A new simulation tool called Atarraya has been developed for testing, implementing and teaching topology control protocols in wireless sensor networks. This is the first simulation tool that works based on the divided definition of topology control. The appendix of this dissertation is dedicated to the detailed description of Atarraya, including the simulator's internal structure, how the protocols were designs and a list of the considerations for an effective use of the tool. The code of Atarraya 
is available to the public under GPL licensing at http://www.cse.usf.edu/

〜labrador/Atarraya.

\subsection{Future Work}

As expected, this dissertation cannot cover all possible insights from all the areas presented. Some of the areas in which there could be some extension are the following:

- A more detailed analysis of the mixed integer programming solution for the minimum connected dominating set (MCDS) problem is required to minimize as much as possible the size of the problem definition and the execution time. In addition, an extension of this problem in order to consider coverage would be very beneficial to the area of coverage-oriented topology construction.

- The testing of the family of A3-based protocols under more realistic scenarios that may consider different communication, sensing and energy models, heterogeneous and mobile networks, and different MAC and routing protocols. In addition, a more detailed analysis of the impact of the weights in the selection metric could provide more insight on how to calculate those weights in order to extend the lifetime of the network even more.

- This dissertation only considers topology maintenance protocols that use energy or time as triggering criteria. The consideration of other triggers and their performance evaluation against the current ones is part of a logical sequence that will enrich this area of research.

- The simulation tool Atarraya is still a project under development. From the implementation of more topology construction and maintenance protocols, along with 
sensor-data management and routing schemes, to the simulation of environmental events to evaluate the real performance and accuracy of the protocols tested; these areas could be investigated with respective extensions in Atarraya. In addition, a more efficient structure to allow faster simulations is also one of the medium term goals after this dissertation. 


\section{List of References}

[1] M. Labrador and P. Wightman, Topology Control in Wireless Sensor Networks. New York, NY: Springer Science + Business Media B.V., 2009.

[2] K. Holger and A. Willig, Protocols and Architectures for Wireless Sensor Networks. John Wiley and Sons, 2005.

[3] M. Penrose, Random Geometric Graphs. Oxford University Press, 2003.

[4] P. Santi, Topology Control in Wireless Ad Hoc and Sensor Networks. $\quad$ England: John Wiley and Sons, 2005.

[5] D. Li and H. Liu, "Sensor coverage in wireless sensor networks," in Wireless Networks: Research, Technology and Applications, J. Feng, Ed. Nova Science Publishers, 2009, pp. 3-31.

[6] M. Penrose, "The Longest Edge of a Random Minimal Spanning Tree," The Annals of Applied Probability, vol. 7, no. 2, pp. 340-361, 1997.

[7] L. Holst, "On Multiple Covering of a Circle with Random Arcs," Applied Probability, vol. 17, pp. 284-290, 1998.

[8] M. Penrose, "A Strong Law for the Largest Nearest-Neighbour Link between Random Points," Journal of London Mathematical Society, vol. 60, no. 2, pp. 951960, 1999. 
[9] P. Santi and D. Blough, "The critical transmitting range for connectivity in sparse wireless ad hoc networks," IEEE Transactions on Mobile Computing, vol. 2, no. 1, pp. 25-39, 2003.

[10] L. Kirousis, E. Kranakis, D. Krizanc, and A. Pele, "Power Consumption in Packet Radio Networks," Theoretical Computer Science, vol. 243, pp. 289-305, 2000.

[11] A. Clementi, P. Penna, and R. Silvestri, "Hardness Results for the Power Range Assignment Problem in Packet Radio Networks," in Proceedings of 3rd.. International Workshop on Approximation Algorithms for Combinatorial Optimization Problems: Randomization, Approximation, and Combinatorial Algorithms and Techniques, 1999, pp. 197-208.

[12] G. Toussaint, "The Relative Neighborhood Graph of a Finite Planar Set," Pattern Recognition, vol. 12, pp. 261-268, 1980.

[13] K. R. Gabriel and R. R. Sokal, “A New Statistical Approach to Geographic Variation Analysis,” Systematic Zoology, vol. 18, pp. 259-270, 1969.

[14] B. Delaunay, "Sur la sphère vide," Izvestia Akademii Nauk SSSR, Otdelenie Matematicheskikh i Estestvennykh Nauk, vol. Vol. 7, pp. 793-800, 1934.

[15] B. Karp and H. T. Kung, "Greedy Perimeter Stateless Routing for Wireless Networks," in Proceedings of ACM/IEEE Mobicom, 2000, pp. 243-254.

[16] X.-Y. Li, G. Calinescu, and P.-J. Wan, "Distributed Construction of a Planar Spanner and Routing for Ad Hoc Wireless Networks," in Proceedings of IEEE Conference on Computer Communications, 2002, pp. 1268-1277.

[17] N. Li, J. C. Hou, and L. Sha, "Design and Analysis of an MST-based Topology Control Algorithm," in Proceedings of IEEE Conference on Computer Communications, 2003, pp. 1702-1712.

[18] V. Rodoplu and T. H. Meng, "Minimum Energy Mobile Wireless Networks," IEEE Journal of Selected Areas in Communications, vol. 17, no. 8, pp. 1333-1344, 1999. 
[19] N. Patwari, J. Ash, S. Kyperountas, A. Hero, R. Moses, and N. Correal, "Locating the Nodes," IEEE Signal Processing Magazine, vol. July, pp. 54-69, 2005.

[20] A. H. Sayed, A. Tarighat, and N. Khajehnouri, "Network-Based Wireless Localization,” IEEE Signal Processing Magazine, vol. July, pp. 24-40, 2005.

[21] G. Sun, J. Chen, W. Guo, and K. J. R. Liu, "Signal Processing Techniques in Network-Aided Positioning," IEEE Signal Processing Magazine, vol. July, pp. 1223, 2005.

[22] A. C. Yao, "On Constructing Minimum Spanning Trees in K-Dimensional Spaces and Related Problems," Journal in Computing of the Society for Industrial and Applied Mathematics, vol. 11, no. 4, pp. 721-736, 1982.

[23] L. Li, J. Y. Halpern, P. Bahl, Y. M. Wang, and R. Wattenhofer, “A Cone-Based Distributed Topology-Control Algorithm for Wireless Multi-Hop Networks," IEEE/ACM Transactions on Networking, vol. 13, no. 1, pp. 147-159, 2005.

[24] S. A. Borbash and E. H. Jennings, "Distributed Topology Control Algorithm for Multihop Wireless Networks," in Proceedings of the IEEE International Joint Conference on Neural Networks, 2002, pp. 355-360.

[25] E. Gelal, G. Jakllari, N. Young, and S. V. Krishnamurthy, "An Integrated Scheme for Fully-Directional Neighbor Discovery and Topology Management in Mobile Ad Hoc Networks," in Proceedings of IEEE International Conference on Mobile Ad Hoc and Sensor Systems, 2006, pp. 139-149.

[26] L. Kleinrock and J. A. Silvester, "Optimum Transmission Radii for Packet Radio Networks or Why Six is a Magic Number," in Proceedings of the IEEE National Telecommunication Conference, 1978, pp. 4.3.1-4.3.5.

[27] J. Ni and S. Chandler, "Connectivity Properties of a Random Radio Network," IEE Proceedings Communications, vol. 141, no. 4, pp. 289-296, 1994. 
[28] T. Hou and V. Li, "Transmission Range Control in Multihop Packet Radio Networks," IEEE Transactions on Communications, vol. 34, no. 1, pp. 38-44, 1986.

[29] F. Xue and P. R. Kumar, "The Number of Neighbors Needed for Connectivity of Wireless Networks," Wireless Networks, vol. 10, no. 2, pp. 169-181, 2004.

[30] D. M. Blough, M. Leoncini, G. Resta, and P. Santi, "The K-Neigh Protocol for Symmetric Topology Control in Ad Hoc Networks," in Proceedings of the 4th ACM International Symposium on Mobile Ad Hoc Networking and Computing, 2003, pp. 141-152.

[31] R. Wattenhofer and A. Zollinger, "XTC: A Practical Topology Control Algorithm for Ad-Hoc Networks," in Proceedings of the 18th International Parallel and Distributed Processing Symposium, 2004.

[32] S. Narayanaswamy, V. Kawadia, R. Sreenivas, and P. Kumar, "Power Control in Ad Hoc Networks: Theory, Architecture, Algorithm and Implementation of the COWPOW Protocol," in Proceedings of the European Wireless Conference, 2002, pp. 156-162.

[33] N. Li and J. Hou, “Topology Control in Heterogeneous Wireless Networks: Problems and Solutions," in Proceedings of IEEE Conference on Computer Communications, 2004.

[34] R. Zhang and M. A. Labrador, "Energy-aware Topology Control in Heterogeneous Wireless Multi-hop Networks," in Proceedings of 2nd. IEEE International Symposium on Wireless Pervasive Computing, 2007.

[35] M. Garey and D. Johnson, Computers and Intractability: A Guide to the Theory of NP-Completeness. W. H. Freeman, 1979.

[36] P. Wan, K. Alzoubi, and O. Frieder, "Distributed construction of connected dominating set in wireless ad hoc networks," in Proceedings of the 21st IEEE Conference on Computer Communications, 2002. 
[37] K. Mnif, B. Rong, and M. Kadoch, "Virtual backbone based on mcds for topology control in wireless ad hoc networks," in Proceedings of the 2nd ACM international workshop on Performance evaluation of wireless ad hoc, sensor, and ubiquitous networks, 2005, pp. 230-233.

[38] W. Duckworth and B. Mans, "On the connected domination number of random regular graphs," in Proceedings of the 8th Annual International Conference on Computing and Combinatorics, 2002, pp. 1-5.

[39] W. Duckworth and N. Wormald, "Minimum independent dominating sets of random cubic graphs," Random Structures and Algorithms, vol. 21, no. 2, pp. 147$161,2002$.

[40] B. Darties, F. Theoleyre, and A. Duda, "A divide-and-conquer scheme for assigning roles in multi-channel wireless mesh networks," in Proceedings of the IEEE Conference on Local Computer Networks, 2009.

[41] S. Guha and S. Khuller, "Approximation algorithms for connected dominating sets," in Proceedings of 4th European Symposium on Algorithms, 1996, pp. 179193.

[42] M. Thai, N. Zhang, R. Tiwari, and X. Xu, "On approximation algorithms of kconnected m-dominating sets in disk graphs," Theoretical Computer Science, vol. 385, no. 1-3, pp. 49-59, 2007.

[43] B. Gao, Y. Yang, and H. Ma, "A new distributed approximation algorithm for constructing minimum connected dominating sets in wireless ad hoc networks," International Journal of Communication Systems, vol. 18, no. 8, pp. 743-762, 2005.

[44] Z. Yuanyuan, X. Jia, and H. Yanxiang, "Energy efficient distributed connected dominating sets construction in wireless sensor networks," in Proceeding of the ACM International Conference on Communications and Mobile Computing, 2006, pp. 797-802. 
[45] S. Funke, A. Kesselman, U. Meyer, and M. Segal, "A simple improved distributed algorithm for minimum cds in unit disk graphs," ACM Transactions in Sensor Networks, vol. 2, no. 3, pp. 444-453, 2006.

[46] R. Prim, "Shortest Connection Networks and Some Generalizations," Bell System Technical Journal, vol. 36, pp. 1389-1401, 1957.

[47] B. Das and V. Bharghavan, "Routing in Ad-Hoc Networks Using Minimum Connected Dominating Sets," in Proceedings of IEEE International Conference on Communications, vol. 1, 1997, pp. 376-380.

[48] S. Parthasarathy and R. Gandhi, "Fast Distributed Well Connected Dominating Sets for Ad Hoc Networks," University of Maryland, Tech. Rep. CS-TR-4559, 2004. [Online]. Available: http://citeseer.ist.psu.edu/parthasarathy04fast.html

[49] B. Chen, K. Jamieson, H. Balakrishnan, and R. Morris, "Span: An Energy-Efficient Coordination Algorithm for Topology Maintenance in Ad Hoc Wireless Networks," Wireless Networks, vol. 8, no. 5, pp. 481-494, 2002.

[50] V. Kumar, T. Arunan, and N. Balakrishnan, "E-span: Enhanced-span with directional antenna," in Proceedings of IEEE Conference on Convergent Technologies for Asia-Pacific Region, vol. 2, 2002, pp. 675-679.

[51] I. Stojmenovic, M. Seddigh, and J. Zunic, "Dominating sets and neighbor elimination-based broadcasting algorithms in wireless networks," IEEE Transactions on Parallel and Distributed Systems, vol. 13, pp. 14-25, 2002.

[52] J. Wu and H. Li, "On Calculating Connected Dominating Set for Efficient Routing in Ad Hoc Wireless Networks," in Proceedings of the 3rd. ACM International Workshop on Discrete Algorithms and Methods for Mobile Computing and Communications, 1999, pp. 7-14.

[53] J. Wu and F. Dai, "An Extended Localized Algorithm for Connected Dominating Set Formation in Ad Hoc Wireless Networks," IEEE Transactions on Parallel and Distributed Systems, vol. 15, no. 10, pp. 908-920, 2004. 
[54] J. Wu, M. Cardei, F. Dai, and S. Yang, "Extended Dominating Set and Its Applications in Ad Hoc Networks Using Cooperative Communication," IEEE Transactions on Parallel and Distributed Systems, vol. 17, no. 8, pp. 851-864, 2006.

[55] W. Heinzelman, A. Chandrakasan, and H. Balakrishnan, "Energy-Efficient Communication Protocol for Wireless Microsensor Networks," in Proceedings of the 33rd International Conference on System Sciences (HICSS), 2000, pp. 1-10.

[56] O. Younis and S. Fahmy, "HEED: A Hybryd, Energy-Efficient, Distributed Clustering Approach for Ad Hoc Sensor Networks," IEEE Transactions on Mobile Computing, vol. 3, no. 4, pp. 366-379, 2004.

[57] A. Cerpa and D. Estrin, "Ascent: Adaptive self-configuring sensor networks topologies," IEEE Transactions on Mobile Computing, vol. 3, no. 3, pp. 272-285, 2004.

[58] G. Xing, C. Lu, Y. Zhang, Q. Huang, and R. Pless, "Minimum Power Configuration for Wireless Communication in Sensor Networks," ACM Transactions on Sensor Networks, vol. 3, no. 2, 2007.

[59] V. Kawadia and P. Kumar, "Power Control and Clustering in Ad Hoc Networks," in Proceedings of IEEE Conference on Computer Communications, 2003, pp. 459469.

[60] L. Bao and J. J. Garcia-Luna-Aceves, "Topology Management in Ad Hoc Networks," in Proceedings of the 4th ACM International Symposium on Mobile Ad Hoc Networking and Computing, 2003, pp. 129-140.

[61] M. Busse, T. Haenselmann, and W. Effelsberg, "TECA: A Topology and Energy Control Algorithm for Wireless Sensor Networks," in Proceedings of the 9th ACM International Symposium on Modeling Analysis and Simulation of Wireless and Mobile Systems, 2006, pp. 317-321. 
[62] Y. Wang and X. Li, "Geometric Spanners for Wireless Ad Hoc Networks," in Proceedings of 22nd. International Conference on Distributed Computing Systems, 2002, pp. 171-178.

[63] J. Wu and F. Dai, "Virtual Backbone Construction in MANETs Using Adjustable Transmission Ranges," IEEE Transactions on Mobile Computing, vol. 5, no. 9, pp. 1188-1200, 2006.

[64] H. Zhang and J. Hou, "Maintaining sensing coverage and connectivity in large sensor networks," Department of Computer Science, University of Illinois at Urbana-Champaign, Technical Report UIUCDCS-R-2003-2351, 2003.

[65] - "Maintaining sensing coverage and connectivity in large sensor networks," Ad Hoc \& Sensor Wireless Networks, vol. 1, no. 1-2, pp. 89-123, 2005.

[66] X. Wang, G. Xing, Y. Zhang, C. Lu, R. Pless, and C. Gill, "Integrated coverage and connectivity configuration in wireless sensor networks," in Proceedings of the 1st international conference on Embedded networked sensor systems, 2003, pp. 28-39.

[67] S. Kumar, T. Lai, and J. Balogh, "On k-coverage in a mostly sleeping sensor network," in Proceedings of the 10th annual international conference on Mobile computing and networking, 2004, pp. 144-158.

[68] J. Carle, A. Gallais, and D. Simplot-Ryl, "Preserving area coverage in wireless sensor networks by using surface coverage relay dominating sets," in Proceedings of the 10th IEEE Symposium on Computers and Communications, 2005, pp. 347-352.

[69] R. Iyengar, K. Kar, and S. Banerjee, "Low-coordination topologies for redundancy in sensor networks," in Proceedings of the 6th ACM International Symposium on Mobile Ad Hoc Networking and Computing, 2005, pp. 332-342.

[70] Y. Wu, M. Li, Z. Cai, and E. Zhu, "A distributed algorithm to approximate node-weighted minimum $\alpha$-connected $(\theta, \mathrm{k})$-coverage in dense sensor networks," in Proceedings of the 2nd annual international workshop on Frontiers in Algorithmics, 2008, pp. 221-232. 
[71] M. Cardei and J. Wu, "Energy-efficient coverage problems in wireless ad-hoc sensor networks," Computer Communications, vol. 29, no. 4, pp. 413-420, 2006.

[72] X. Bai, D. Xuan, Z. Yun, T. Lai, and W. Jia, "Complete optimal deployment patterns for full-coverage and k-connectivity $(\mathrm{k} \leq 6)$ wireless sensor networks," in Proceedings of the 9th ACM international symposium on Mobile ad hoc networking and computing, 2008, pp. 401-410.

[73] S. Shakkottai, R. Srikant, and N. Shroff, "Unreliable Sensor Grids: Coverage, Connectivity and Diameter," in Proceedings of IEEE Conference on Computer Communications, 2003, pp. 1073-1083.

[74] X. Bai, S. Kumar, D. Xuan, Z. Yun, and T. Lai, "Deploying wireless sensors to achieve both coverage and connectivity," in Proceedings of ACM Symposium on Mobile Ad Hoc Networking and Computing, 2006, pp. 131-142.

[75] Y. Hou, T. Lee, C. Chen, and B. Jeng, "Node placement for optimal coverage in sensor networks," in Proceedings of IEEE International Conference on Sensor Networks, Ubiquitous, and Trustworthy Computing, 2006, pp. 352-357.

[76] R. L. Graham and B. D. Lubachevsky, "Repeated patterns of dense packings of equal disks in a square," Electronic Journal of Combinatorics, vol. 3, no. 1, pp. $1-16,1996$.

[77] R. Kershner, "The number of circles covering a set," American Journal of Mathematics, vol. 61, no. 3, pp. 665-671, 1939.

[78] T. Aste and D. Weaire, The Pursuit of Perfect Packing. Institute of Physics Publishing, 2000.

[79] M. Bagheri, M. Hefeeda, and H. Ahmadi, "A near optimal k-coverage algorithm for large-scale sensor networks," Simon Fraser University, School of Computing Science, Technical Report TR 2006-10, 2006. [Online]. Available: ftp://fas.sfu.ca/pub/cs/TR/2006/CMPT2006-10.pdf 
[80] V. Fodor and I. Glaropoulos, "On the gains of deterministic placement and coordinated activation in sensor networks," in Proceedings of IEEE Globecom, 2008, pp. 1-6.

[81] B. Wang, K. Chua, and V. Srinivasan, "Connected sensor cover for area information coverage in wireless sensor networks," International Journal of Communication Systems, vol. 21, no. 11, pp. 1181-1203, 2008.

[82] K. Yildirim, T. Kalaycir, and A. Uğur, "Optimizing coverage in a k-covered and connected sensor network using genetic algorithms," in Proceedings of the 9th WSEAS International Conference on Evolutionary Computing, 2008, pp. 21-26.

[83] A. Konstantinidis, K. Yang, and Q. Zhang, "An evolutionary algorithm to a multiobjective deployment and power assignment problem in wireless sensor networks," in Proceedings of IEEE GLOBECOM, 2008, pp. 1-6.

[84] F. Ye, G. Zhong, J. Cheng, L. Songwu, and L. Zhang, "Peas: A robust energy conserving protocol for long-lived sensor networks," in Proceedings of the 23rd International Conference on Distributed Computing Systems, 2003, pp. 28-37.

[85] Y. Cai, M. Li, W. Shu, and M. Wu, "Acos: An area-based collaborative sleeping protocol for wireless sensor networks," Ad Hoc \& Sensor Wireless Networks, vol. 3, no. 1, pp. 77-97, 2007.

[86] S. Zhang, Y. Liu, J. Pu, X. Zeng, and Z. Xiong, "An enhanced coverage control protocol for wireless sensor networks," Proceedings of the Hawaii International Conference on System Sciences, pp. 1-7, 2009.

[87] M. Wueng, S. Hwang, and C. Ho, "Akce: An efficient and accurate k-coverage eligibility algorithm in wireless sensor networks," in Proceedings of the IEEE International Symposium on Modeling, Analysis and Simulation of Computers and Telecommunication Systems, 2008, pp. 1-8.

[88] H. Bai, X. Chen, B. Li, and D. Han, "A location-free algorithm of energy-efficient connected coverage for high density wireless sensor networks," Discrete Event Dynamic Systems, vol. 17, no. 1, pp. 1-21, 2007. 
[89] D. Tian and N. Georganas, "A node scheduling scheme for energy conservation in large wireless sensor networks," Wireless Communications and Mobile Computing, vol. 3, no. 2, pp. 271-290, 2003.

[90] H. Gupta, Z. Zhou, S. Das, and Q. Gu, “Connected sensor cover: Self-organization of sensor networks for efficient query execution," IEEE/ACM Transactions on Networking, vol. 14, no. 1, pp. 55-67, 2006.

[91] A. Ghosh and S. Das, "A distributed greedy algorithm for connected sensor cover in dense sensor networks," in Proceedings of 1st IEEE International Conference on Distributed Computing in Sensor Systems, 2005, pp. 340-353.

[92] J. Wang and S. Medidi, "Mesh-based coverage for wireless sensor networks," in Proceedings of IEEE GLOBECOM, 2008, pp. 1-5.

[93] A. Datta, M. Gradinariu, and R. Patel, "Distributed self-* minimum connected covering of a query region in sensor networks," in Proceedings of 8th International Symposium on Parallel Architectures, Algorithms and Networks, 2005, pp. 448453.

[94] S. Funke, A. Kesselman, F. Kuhn, Z. Lotker, and M. Segal, "Improved approximation algorithms for connected sensor cover," Wireless Networks, vol. 13, no. 2, pp. 153-164, 2007.

[95] S. Yang, F. Dai, M. Cardei, and J. Wu, "On multiple point coverage in wireless sensor networks," in Proceedings of the IEEE International Conference on Mobile Ad hoc and Sensor Systems Conference, 2005, pp. 757-764.

[96] M. Cardei and D. Du, "Improving wireless sensor network lifetime through power aware organization," Wireless Networks, vol. 11, no. 3, pp. 333-340, 2005.

[97] X. Chu and H. Sethu, "A new distributed algorithm for even coverage and improved lifetime in a sensor network," in Proceedings of IEEE Conference on Computer Communications, 2009, pp. 361-369. 
[98] Y. Zou and K. Chakrabarty, "Fault-Tolerant Self-organization in Sensor Networks," in Proceedings of DCOSS, 2005, pp. 191-205.

[99] L. Frye, L. Cheng, S. Du, and M. Bigrigg, "Topology Maintenance of Wireless Sensor Networks in Node Failure-prone Environments," in Proceedings of the IEEE International Conference on Networking, Sensing and Control, 2006, pp. 308-312.

[100] S. Chessa and P. Santi, "Comparison-based System-level Fault Diagnosis in Ad Hoc Networks," in Proceedings of the 20th IEEE Symposium on Reliable Distributed Systems, 2001, pp. 257-266.

[101] M. Elhadef, A. Boukerche, and H. Elkadiki, "Diagnosing Mobile Ad-hoc Networks: Two Distributed Comparison-based Self-diagnosis Protocols," in Proceedings of the 4th ACM International Workshop on Mobility Management and Wireless Access, 2006, pp. 18-27.

[102] Y. Xu, J. Heidemann, and D. Estrin, "Geography-informed Energy Conservation for Ad Hoc Routing," in Proceedings of the 7th Annual International Conference on Mobile Computing and Networking, 2001, pp. 70-84.

[103] _ _ "Adaptive Energy-Conserving Routing for Multihop Ad Hoc Networks," USC/Information Sciences Institute, Research Report 527, October 2000. [Online]. Available: http://www.isi.edu/ johnh/PAPERS/Xu00a.html

[104] T. Haynes, S. Hedetniemi, and P. Slater, Fundamentals of Domination in Graphs. Marcel Dekker, Inc., 1998.

[105] D. Yuan, "Energy-efficient broadcasting in wireless ad hoc networks: performance benchmarking and distributed algorithms based on network connectivity characterization," in Proceedings of the 8th ACM international symposium on Modeling, analysis and simulation of wireless and mobile systems, MSWiM. New York, NY, USA: ACM, 2005, pp. 28-35.

[106] P. Wightman and M. Labrador, "A3: A topology control algorithm for wireless sensor networks," in Proceedings of IEEE Globecom, 2008, pp. 1-6. 
[107] — - "Atarraya: A simulation tool to teach and research topology control algorithms for wireless sensor networks," in Proceedings of the 2nd International ICST Conference on Simulation Tools and Techniques, March 2009.

[108] J. Broch, D. Johnson, and D. Maltz, "The dynamic source routing protocol for multihop wireless ad hoc networks," in Ad Hoc Networking, C. Perkins, Ed. Boston, MA, USA: Addison-Wesley, 2001, ch. 5, pp. 139-172. 
Appendices 


\section{Appendix A: A Brief Overview of Atarraya}

\section{Introduction}

The main idea behind the creation of Atarraya - which means fishnet in Spanish - was to test the topology construction protocol named A3 that was being developed as part of this research. The software, as originally conceptualized, was very simple, rigid and tightly coupled with the A3 protocol. However, due to the fact that it was necessary to compare the performance of A3 against other known topology construction mechanisms, the design of the tool was not adequate. Therefore, the decision to build a more generic simulator, in which other topology construction algorithms could be plugged in, and have a single platform where to evaluate them all under the same conditions, was necessary. Then, the concept of topology control was also expanded to include topology maintenance algorithms, and several of these mechanisms were designed and included as well. The final result is Atarraya: a generic, Java-based, event-driven simulator for topology control algorithms in wireless sensor networks. As with any simulation tool born out of a research effort, Atarraya is still in development; however, in its current state, it is an excellent tool not only for research, to develop and test new topology control algorithms, but also for teaching. Atarraya's graphical user interface shows how topology control protocols work, and how they shape topologies during their execution. In addition, Atarraya includes necessary mechanisms to experiment with classic theoretical results related to topology control in wireless sensor networks, such as the giant component experiment, calculation of the critical transmission range (CTR), calculation of the Minimum Spanning Tree of a graph, and others.

In this appendix the basics of Atarraya are presented along with its internal structure, so the reader knows how to develop and plug in new topology control algorithms and proto- 


\section{Appendix A: (continued)}

cols, and a brief guide on how to use the tool. All explanations and descriptions included in this document are related to Atarraya's version 1.0, which is the version that was used to run all the experiments included in the book. Future versions and new features will be documented on the project's Website at http://www.csee.usf.edu/ labrador/Atarraya.

\section{Description of Atarraya's Internal Structure}

In this section the internal structure of Atarraya is described. First, its main functional components and Atarraya's class tree are presented. Then, the structure of the protocols is described in more detail, including how they communicate with the main class and with other protocols, how to initialize the nodes, and how to handle protocol events.

\section{Abstract Design and Functional Components}

This section describes the main functional components of the simulator and how they interact among themselves. The functional components offer the "big picture" necessary to understand the critical components of Atarraya. Figure A.1 presents a global view of the internal structure of the simulator, which consists of the main simulator thread, the node handler, and the batch executor. The elements of this structure are described in this section. 


\section{Appendix A: (continued)}

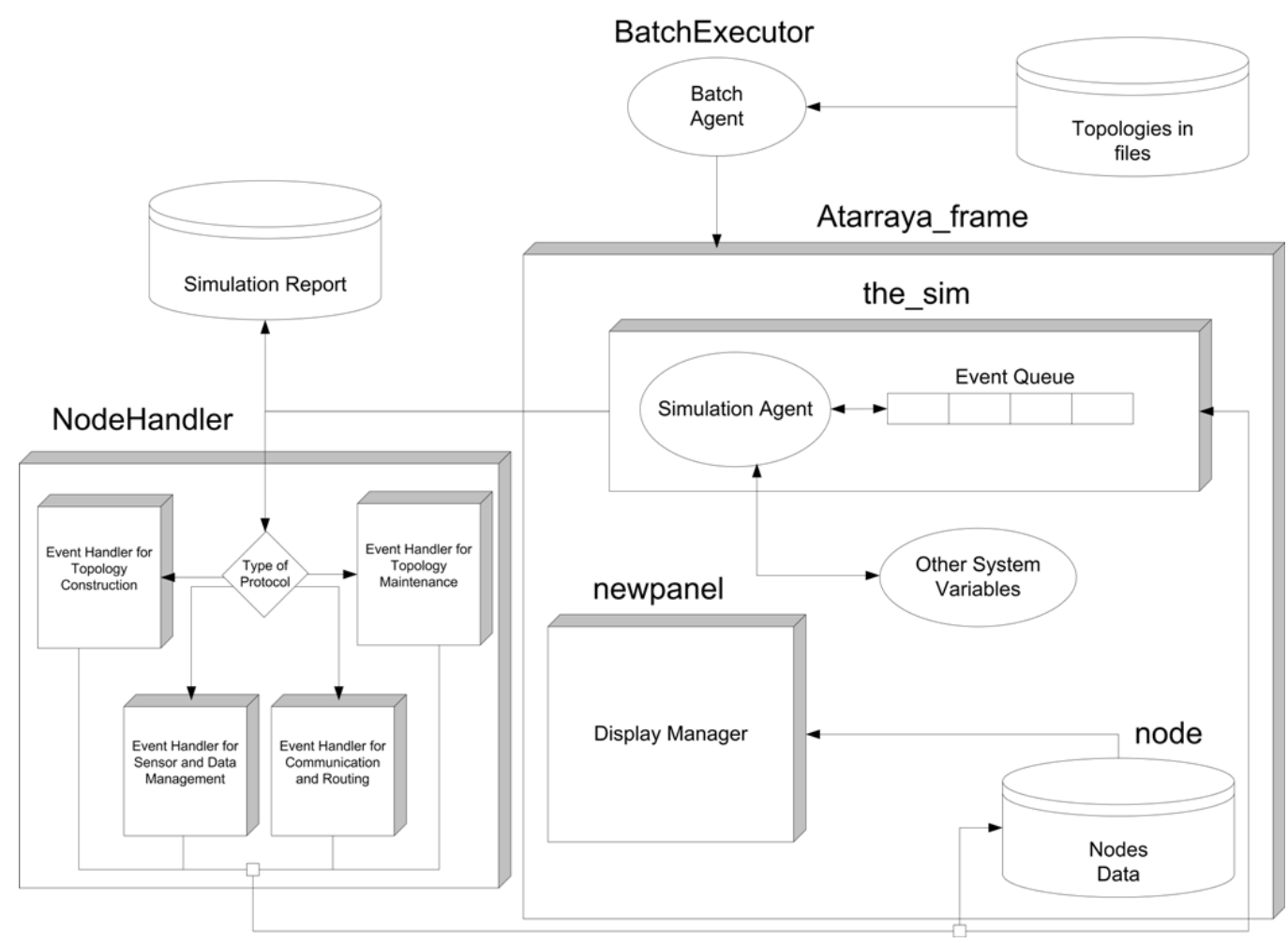

Figure A.1: Atarraya's functional components. 


\section{Appendix A: (continued)}

\section{The Main Simulator Thread - The the_sim Class}

This is the core of the system. The simulator thread, defined in the class the_sim, is in charge of fetching the next event from the simulation event queue, and sending the event to the node handler for execution. An instance of this class is created by the method StartSimulation() whenever a simulation is executed. This class contains the event queue, the simulation clock, the display manager, the database with the data about the nodes, and the simulation agent, which is in charge of storing the simulation results for the reports in the respective logs.

When an instance of the the_sim class is created, it is necessary to add the initial events to the queue before the thread is started. The first thing the thread will do once started is to check if there are any events in the Event Queue. If the thread is started without any events, it will consider that an error has occurred, and the simulation will be suspended. Once the first events have been loaded into the queue, the simulator thread can get started. The thread starts a loop that will execute until one of the three termination conditions is true: there are no events in the queue, all the nodes have reached the final state in the topology control protocol, or the protocols have called for the end of the simulation (for example, the topology maintenance protocol has found that the sink has no more neighbors, so the network is dead). If the first condition occurs and the simulator has not been notified that the protocols finished execution, it means that there was an error during the simulation, and it will be notified on the simulation report.

In the loop, the first thing the thread does is to verify if the event is valid. If so, the event will be registered (if this option was selected by the user), the simulation clock will be 


\section{Appendix A: (continued)}

updated, and the event will be sent to the NodeHandler. There, the event will be delivered to the appropriate EventHandler according to the respective protocol the event belongs to. Once the event is executed, the simulator will go back to the loop and start again the process. The simulator updates the clock with the execution time of the events based on the fact that all the events in the queue are sorted by their projected execution time, so there is no such thing like a trip to the past.

Once the simulator breaks the loop by any of the finalization conditions mentioned above, the thread goes to the report construction section, saving all the events and statistics, as selected by the user. This section also takes into account whether or not the simulation is part of a batch execution, in which case all the data from all previous executions is kept until the last one finishes. All this information is stored in data structures that are stored in the report files after the simulation is finished. Reading this section of the code will provide the user with information about all the options for each configuration of report in both single and batch simulation cases. Once the simulation and the report building section finish, the thread ends too.

In the current version of the simulator, just one simulator thread can run at a time because there is only one data structure to store the topology, which is localized in the atarraya_frame class. Individual instances of the data structure running several simulations in parallel will consume all the resources of the Java virtual machine, especially if the network topologies are big. 


\section{Appendix A: (continued)}

\section{The Protocol Manager - The NodeHandler Class}

This class is in charge of defining the protocols to be used in the simulation and routing the event to the appropriate protocol once received from the simulation thread. The NodeHandler class defines the four possible protocols that a node can have running during a simulation: Topology construction, topology maintenance, sensor-data management, and communication-routing protocols. Given that there are different algorithms for each type of protocol, the main purpose of this class is to make that selection transparent to the rest of the simulator, so that no detail about the selection is required in order to execute the simulation. When a simulation is started, this class creates the instances of the selected protocols in each of the four different categories. The simulation thread sends the next event from the event queue to the NodeHandler class. Once the event is received, it is routed to the appropriate protocol based on the protocol identifier included in the event.

\section{The Multiple Operation Thread - The BatchExecutor Class}

The main purpose of the BatchExecutor thread is to perform operations that require multiple executions, such as creating a set of topologies, performing a large number of simulations, and the Giant Component test. Since these operations are run on a thread independent from the main one, the graphical user interface does not freeze while these operations are being executed, which allows for the interaction between the user and the simulator even while some of these operations are running in the background. This class is instantiated whenever one of the mentioned operations is started. 


\section{Appendix A: (continued)}

\section{The Display Manager - The newpanel Class}

The display manager, or newpanel class, is the one in charge of the graphical representation of the topologies. The heart of this class is the override of the Paint method of this class that extends a Panel class. All the painting options for the topology are defined in this method. The other methods perform minor but necessary actions like obtaining information about the options, providing coordinates from the deployment area, etc. This class was defined as a private class of the atarraya_frame class so it can have direct access to the topology data structure.

Atarraya provides several options for topology visualization, which can be seen in more detail in the visualization options in Figure A.12. The most relevant visualization options are the following:

- MaxPower Topology: This is the original view of the topology with all nodes transmitting at full power, and all the links that their unit disks provide.

- Single selected network configuration or tree: In this view the user defines which of the Virtual Network Infrastructures (VNI) he or she wants to see. More on VNI later in Section A. The default configuration is Black in most protocols.

- All network configurations: If several configurations are defined in a certain topology, this view allows the user to see all of them and appreciate the differences between them. 


\section{Appendix A: (continued)}

- Active network configuration: Each node is assumed to be able to maintain several VNIs, but use only one at a time. This view allows the user to see in real-time in which network configurations the nodes of the topology are.

\section{Atarraya's Class Tree}

In this section the class tree of Atarraya is described and a brief explanation of the structure and mission of the current internal structure of the application is provided. The classes in Atarraya are organized in three packages:

- Atarraya: the main functional elements are stored here, such as the main frame, the simulation agent, and the display manager.

- Atarraya.element: This package contains the classes that model the data structures, like the node, VNI, routing table, etc.

- Atarraya.event: This package contains the classes related to the protocols and the definition of the event queue.

\section{The Atarraya Package}

The Atarraya package is is the main package of the simulator. It contains the following classes:

- Main class: This is the launcher of the simulator. It invokes the title frame and the main frame. 


\section{Appendix A: (continued)}

- atarraya_frame class: This is the main class of the simulator. This class contains the graphical userinterface and the simulator core.

- newpanel private class: This class defines the operation related to the visualization panel for the topologies: painting, selection of coordinates, selection of nodes, grid, etc. It is a private class of the atarraya_frame class so the newpanel class has direct access to the data structures.

- the_sim private class: This class defines the structure of the thread that simulates a scenario; in other words, this class is the simulation executor. It was made private also to preserve the direct access to the data structures.

- BatchExecutor class: This class is in charge of executing operations that involve multiple scenarios, being that create multiple topologies, or simulate multiple scenarios. The advantage of using a separate class is that it creates a different thread that freezes the main frame while executing.

- constants interface: This interface defines the standard values for multiple variables. Given that many classes must share a set of standard values, the use of the constant interface allows this without having to define identical variables on each class.

- FrameLogo class: Initial frame with the logo of the simulator.

- AboutFrame class: Frame that contains the "About us" message. 


\section{Appendix A: (continued)}

\section{The Atarraya.element Package}

This package contains the classes that define the elements that will be used in the simulator for storing information. These classes are:

- node class: This class represents all the information about a single node and all the operations that can be performed on it.

- register class: This class contains the information that the simulator has about the neighbors of a node. There is a difference between the information that the node has about its neighbors and the information that the simulator has about them. For example, the simulator needs to know the exact position of each node, but maybe the node does not have the ability to know the position of its neighbors.

- candidate class: This class contains the information that the node has about its own neighbors. This class has two data structures: candidate list and children list. Both are general use data structures on the node.

- NodeNetworkConf class: Based on the assumption that each node may have different VNI, this class represents everything the node needs to know from its current network: list of neighbors, list of gateways, sink's address, routing table, etc. A node can have as many different VNI as desired. By default, the maximum number of VNI is 5 and every node is in the VNI 0 and will remain there until something else is defined. 


\section{Appendix A: (continued)}

- NodeSensingConf class: This class is similar to the NodeNetworkConf class, but applied to the sensing device. Each node may have several sensing devices or different configurations. This class has not been fully implemented.

- routing_table class: This class defines the data structure and methods of the routing table of a VNI of a node. The main structure is a vector of routing_table_register elements. Examples of functionalities of this class are obtaining the appropriate gateway node for sending a packet through the best route, adding routes, storing message sequence numbers, etc.

- routing_table_register class: This class defines the format of a register of the routing table. It includes the address of the destination node, the ID of the next hop and routing metric, and the vector for storing the history of message sequence numbers, which are important for routing algorithms.

- TMStructConfList class: Some topology maintenance protocols require a great amount of information, especially those that can run several processes in parallel. This class models a data structure that implements a list of the independent TMConfStructure instances that a node is handling at a certain moment in time. The concept is similar to the one of the VNI, but just applies to the topology maintenance protocol.

- TMConfStructure class: This class defines the information that a node has about a single topology maintenance process, such as the identifier, the node's state on the topology maintenance process, and a routing table that could be used when specific paths must be restored. 


\section{Appendix A: (continued)}

- pair class: A class that defines a pair of integer values.

- trio class: A class that defines a set of three integer values.

- edge class: A class that defines an edge (source, destination, and weight). This class is used to calculate the MST of a graph.

\section{The Atarraya.event Package}

This package contains the classes that define the structure of the events, the event queue, and the event handlers that will execute the events accordingly. The main classes are:

- event_sim class: This class defines the information of an event: source, destination, type of event, embedded information, configuration, layer that generated the event, etc. This class contains all the information that the event handler requires to execute the event properly.

- eventQueue class: This class defines the queue of events that Atarraya uses during the execution of a simulation. Events are added to the queue in an organized way, based on the projected execution time of the event, so the event on the head of the queue is always the closest to the present time.

- EventHandlerxxx class: This is a family of classes that define the protocols. Each class has to define some initialization operations for the nodes, how the events will affect the state of the nodes, the data structures, other events that get triggered as a consequence of the occurrence of one event, etc. A class of this type needs to be designed if a new topology control algorithm is to be included in the simulator, or 


\section{Appendix A: (continued)}

if an existing algorithm is to be modified. In its current version, Atarraya supports four types of protocols: Topology Construction, Topology Maintenance, SensorData Management, and Communication-routing protocols. The way these protocols communicate is by generating events of each other's type.

- NodeHandler class: This class defines a data structure that holds the selected options for the four types of protocols. Any new protocol added to Atarraya must be included in the existing list that this class contains.

\section{Protocol Structure and Design - The EventHandler Class}

This section introduces the design and structure of the protocols in Atarraya. The EventHandler class is the one that models the structure of a protocol in Atarraya. The next subsections describe the types of events that a protocol in Atarraya can model, how states are labeled, how each protocol communicates with the atarraya_frame class, how protocols interact with each other, how nodes are initialized, and how the simulator handles events.

\section{Simulation Events}

Given that Atarraya is an event-driven simulator, everything that happens during a simulation is an event, so protocols must be defined in terms of cause-effect when certain event occurs. Each of these types of events triggers some internal actions in the node that might modify its status, data structures, etc., and could also cause the generation of new events in the future. The most common examples of events in a protocol are: 


\section{Appendix A: (continued)}

\section{Sending Messages}

When a node intends to send a message, it may be addressed to a specific node (unicast) or it may be intented for every neighbor within range (broadcast). Regardless, the method that a node needs to call on in order to send a message is broadcast. The parameters are the time at which the packet is received, the current time, the id of the sender node, the id of the receiver node (if it is a unicast message), the type of message, the payload, and the VNI corresponding to this package. In general, a message of this kind is assumed to be of the same type as that of the protocol that contains it, which explains why there is no specification of the protocol's type. More data can be included in this message, like the first sender of the message or source, and the final destination of the packet, in case it is a message that will travel through multiple hops.

broadcast (temp_clock+getRandom(MAX_TX_DELAY_RANDOM), temp_clock, sender, -1, HELLO, temp_data,temp_vni);

The method broadcast is in charge of generating the reception events in the neighbors within communication range of the sender node, if the recipients and the sender node are active. The method broadcast is defined on the atarraya_frame class.

\section{Receiving Messages}

When a node receives a message, it calls the event determined by the type of message. If the message is supposed to be a unicast transmission, the node verifies if the id of the destination node matches its own. If that is the case it continues with the execution of the 


\section{Appendix A: (continued)}

algorithm. If the receiver and the destination do not match, the node ignores the packet. Now, if the message was designed to be a broadcast transmission or the node needs to snoop in the packets not addressed to itself, it can ignore the receiver destination verification and just continue with the execution of the protocol.

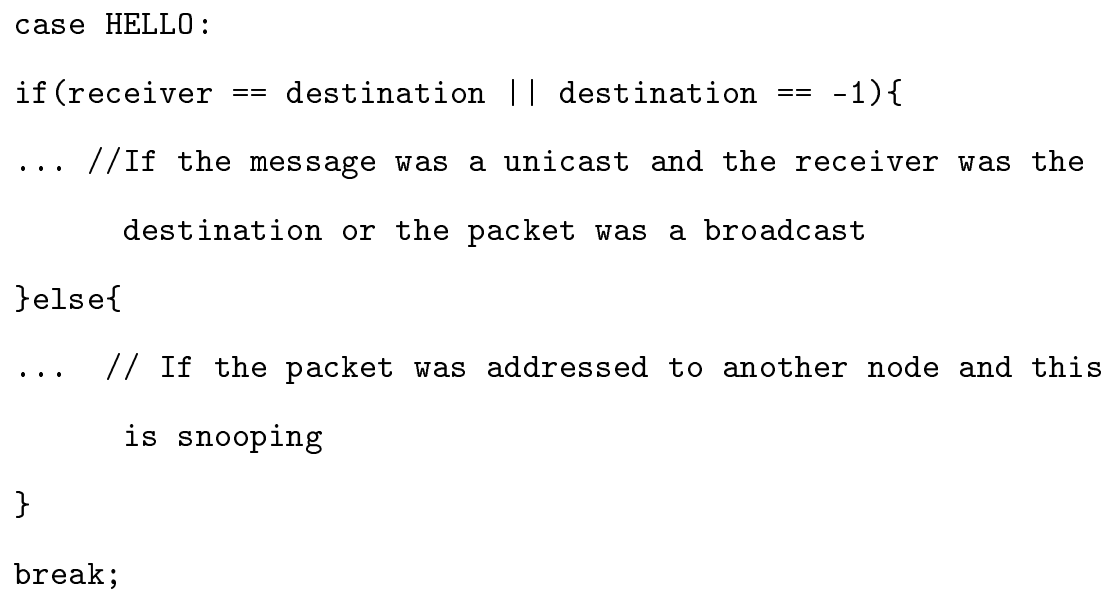

\section{Programming a Timeout}

Sometimes a node needs to wait some time in order to perform a certain action. The definition of a timer is crucial for this type of operation. A timer in Atarraya is an event that a node programs addressed to itself in the future. The parameters are very similar to the ones provided to the broadcast method, with the difference that here the parameters of a new event, that will be included directly in the simulation queue, are also specified. Since this is a reflective event, the sender and the receiver have the same value. Also, in the declaration it is necessary to specify the target protocol of this event in the variable type. In the example, the node sender is programming itself an event of the type PAR$E N T \_R E C O G \_T I M E \_O U T$, that will be executed in TIMEOUT_DELAY time units. 


\section{Appendix A: (continued)}

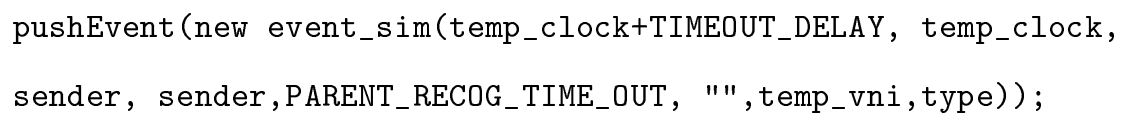

\section{Invalidating a Programmed Event}

A node programs events in the future without knowing what will really happen between the current time and the future event. For example, a node can be programmed to send a message in the future, but for some reason it may also be put to sleep before it can send the message. Since that particular event will not occur, it has to be taken out from the simulation queue, where they are waiting to be executed. Atarraya provides the methods InvalidateAllEvents in order to guarantee that a node can cancel events that should not happen. In the example, the sender node eliminates all the events of the current protocol, referent to the VNI temp_tree from the current time forward.

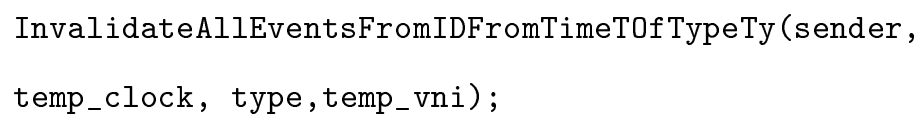

\section{State Labels}

In general, a good number of topology construction protocols use node states to represent the evolution of the protocol. In Atarraya, nodes can be in any of the following four states: Initial, Active, Inactive, and Sleeping states. The definitions of these four states are included in the Node_Handler class. The values defined as the parameters are usually defined in the constants interface, and they are all positive integer values.

tc_protocol_handler.setLabels(S_INITIAL_STATE, S_ACTIVE, 


\section{Appendix A: (continued)}

S_SLEEP, S_SLEEP);

Given that most topology control protocols implemented in Atarraya are completely distributed, the sink cannot call the end of the protocol because it has no information about the state of all the nodes. That is the reason why Atarraya knows that a protocol has finished when all the nodes have reached the final state. Each topology control protocol can define which states are selected as the final states. This is done in the method CheckIfDesiredFinalState(int s) that is defined in every EventHadler, which is invoked in the atarraya_frame class when the simulation agent is trying to verify if the topology control algorithm is finished. In the following example, the protocol is selecting the active and the inactive states as the final states of the nodes.

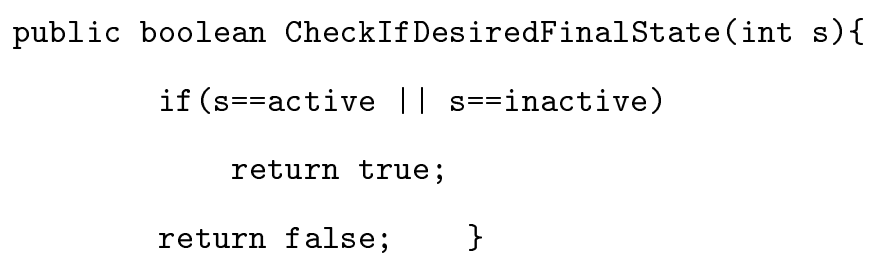

Atarraya stops whenever the nodes of the topology are in any of the selected states, no matter if there are still events in the queue.

\section{Communication with the atarraya_frame Class}

Each protocol receives a reference to the instance of Atarraya's main frame, as defined in the NodeHandler. This reference allows the protocol to have access to variables from the main class. In order to access the variables from the simulator, the protocol needs to use the method father.getVariable(int code), where the parameter code is a defined label for 


\section{Appendix A: (continued)}

the sets of variables that can be accessed. This list can be found in the constants interface, and in the atarraya_frame class where the getVariable() method is defined. For example, if the user wants to know how many nodes are in the topology (including the sink nodes), the following line returns this value:

tam $=$ (int) father.getVariable (NUMPOINTS)

When the protocol needs to get information about a node or modify it, the method to use is getNode(int $i d$ ), where $i d$ is the unique id of the node. In order to set node $i$ in the initial state of the protocol in the VNI_vniID, the following line can be used:

getNode(i).setState(initial,_vniID)

\section{Interaction with Other Protocols}

There will always be some level of communication between protocols. For example, inter-protocol communication is needed to avoid situations like one node wanting to send a data message without having a route to the sink. Given that in Atarraya every event in the simulation goes to the same queue, it is necessary to determine to which protocol it must send the event to. Each type of protocol has its own identifier label, which is included in the event definition. This allows the Node_Handler to send the event to the appropriate protocol.

One of the premises of Atarraya is to create modular protocols that can be used in as many combinations as possible with the other protocols. Accordingly, protocols in Atarraya can only generate events in other protocols. For example, once a node reaches the final state of its topology construction algorithm, it can notify the topology maintenance 


\section{Appendix A: (continued)}

protocol to start the maintenance procedure. Most of the times these inter-protocol events are meant to initiate or stop certain activity, so the protocol and how it works internally are completely independent, but the other protocols can decide the starting points. The following example illustrates a topology construction protocol when it invokes the topology maintenance protocol.

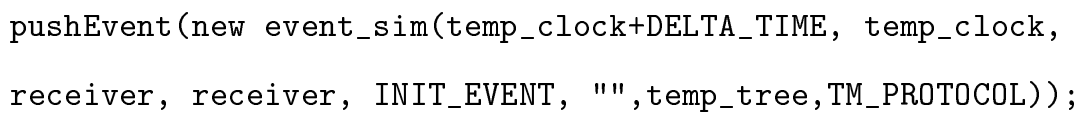

\section{Initialization of Nodes and the Initial Events - The init_nodes and the initial_event Methods}

The init_nodes(int vni) method is used to set the nodes ready to start the execution of the simulation. Nodes are set to their initial states, and any previously defined events regarding other protocols and all necessary variables are set to their default values. This method is invoked in the StartSimulation method in the atarraya_frame class, for all nodes, including the sink. The following code is an example of a init_nodes routine, in which every node is set to its initial state, every state label is defined, any existent programmed event in the queue is cancelled, and the execution of the topology maintenance and sensor and data management protocols are reset.

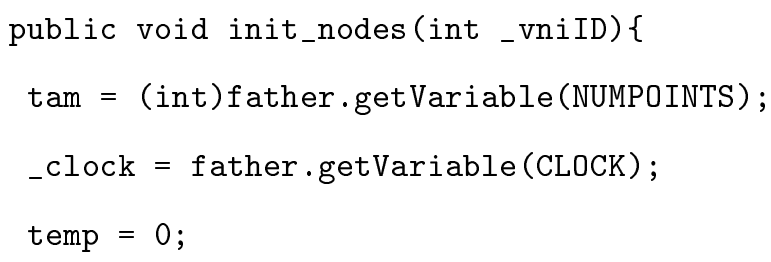




\section{Appendix A: (continued)}

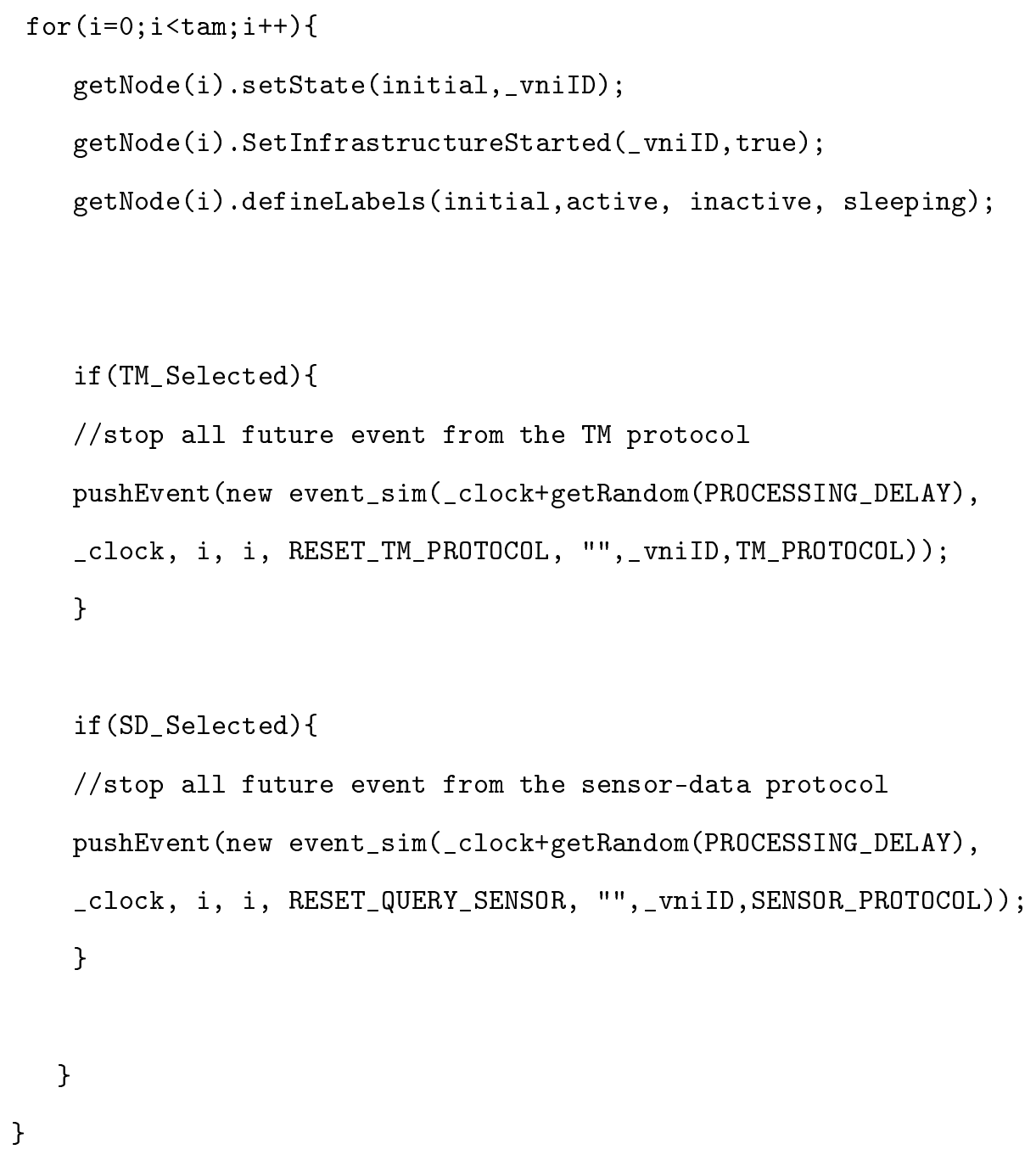

The initial_event(int_id, int_vniID) method is used to define the first events to be included in the queue, before the simulation agent is started. Remember that if the simulation agent finds an empty queue it considers that the simulation has finished in an incorrect way. This method needs two parameters: the ID of the node that will perform the first event, and the VNI ID. This method is also invoked in the StartSimulation() method in the atarraya_frame class, but only for the sink nodes. If no sink nodes are defined in your 


\section{Appendix A: (continued)}

topology, make sure that events are included in the queue using the init_nodes(_vniID) method.

\section{The HandleEvent Method}

This method is the core of the protocol, as it defines the actions taken by the protocol when an event occurs. The unique parameter that this method receives is the event taken from the event queue.

The events are classified based on an event label. Each protocol defines a set of labels for all the events that it uses. These labels are defined in the constants interface. The first action taken by the HandleEvent method is to recover all the fields from the event and store them in temporary variables. Depending on the nature of the protocol, the classification of the events can be done in different ways: Label-then-State or State-then-Label. In the first case, the most important information is the label of the event, which becomes the main classification factor. Once the label is found, the code inside determines if the state of the node is important or not for the execution of the actions associated with the event. In the second case, the most important information is the state of the node. This methodology is useful when there are not many types of events but each type is interpreted differently based on the state of the node.

The following code shows the HandleEvent method from the example protocol presented in this section, the SimpleTree. 


\section{Appendix A: (continued)}

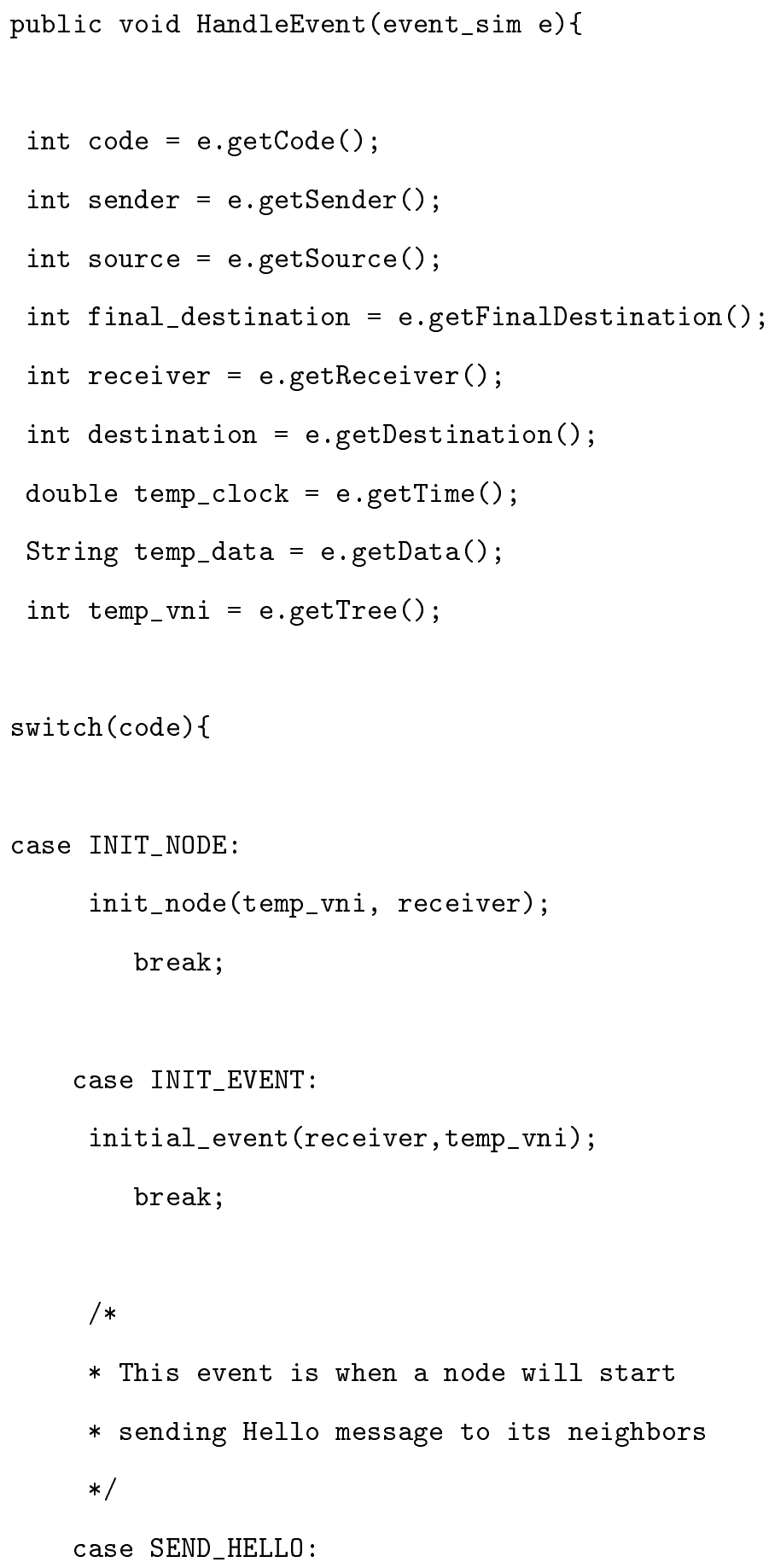




\section{Appendix A: (continued)}

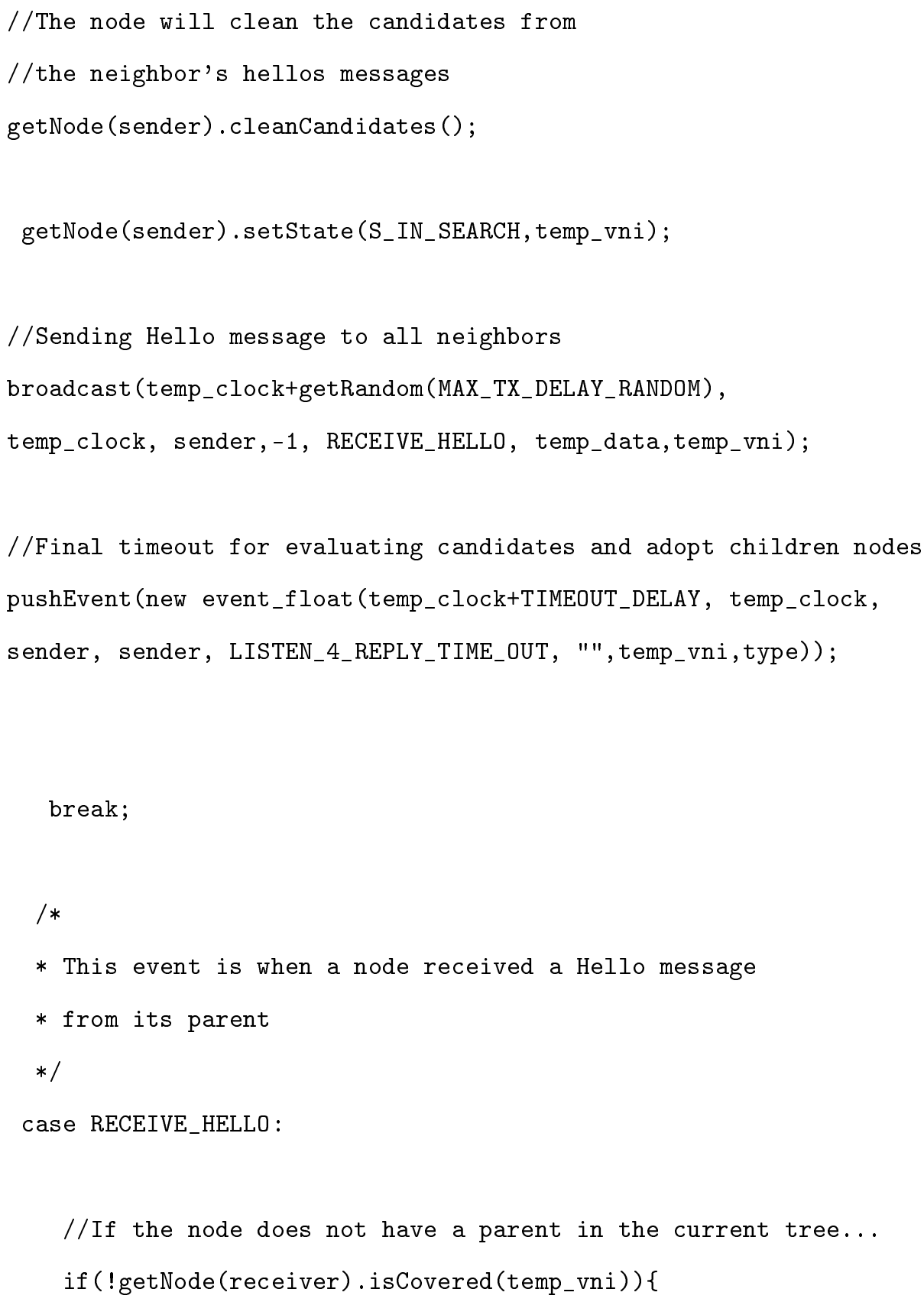




\section{Appendix A: (continued)}

//Decompress the data in the message

temp_data_array = temp_data.split("@");

$\operatorname{try}\{$

//The level of the parent is comming in the data

temp_data_int $=$ Integer. parseInt (temp_data_array $[0])$;

//The sink address of this VNI

temp_data_int 2 = Integer.parseInt (temp_data_array[1]);

\}catch (Exception ex) \{ex.printStackTrace ()$;\}$

//Change the state of the node from the initial state

getNode(receiver) .setState(S_VISITED, temp_vni);

//Define the parent in the current tree and the sink address

getNode(receiver).setParent (temp_vni, sender, sender, temp_data_int2);

// Define the level

getNode(receiver).setLevel (temp_data_int+1);

// Schedules the Broadcast of the Reply message

pushEvent(new event_float(temp_clock+getRandom(PROCESSING_DELAY),

temp_clock,receiver, receiver, SEND_REPLY, "",temp_vni,type));

//If it is not a parent after TIMEOUT_NO_PARENT units,

// it will go into S_SLEEPING mode

pushEvent(new event_float(temp_clock+TIMEOUT_NO_PARENT, temp_clock,

receiver, receiver, END_TIMEOUT_NO_PARENT, "",temp_vni,type));

\} 


\section{Appendix A: (continued)}

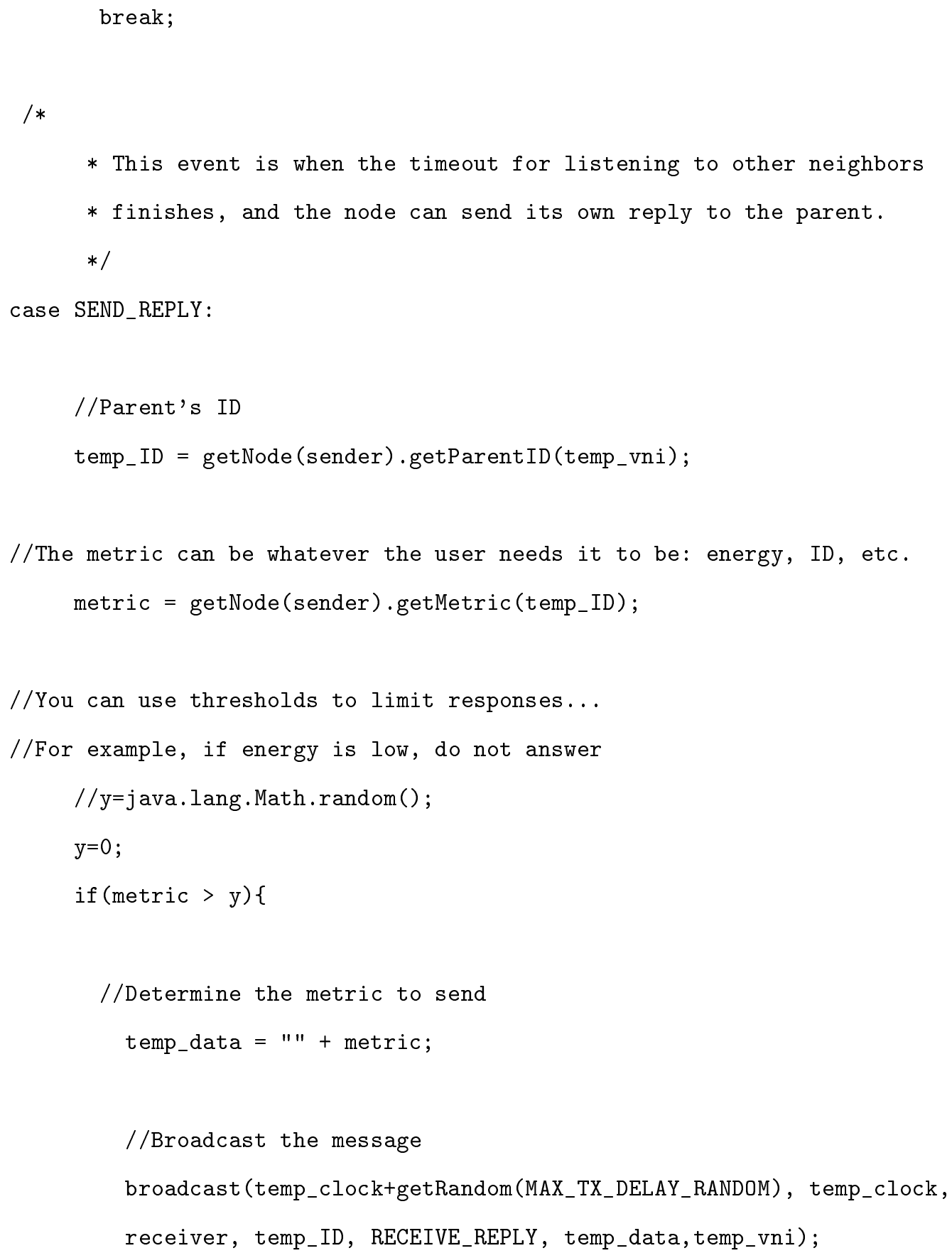




\section{Appendix A: (continued)}

\}

break;

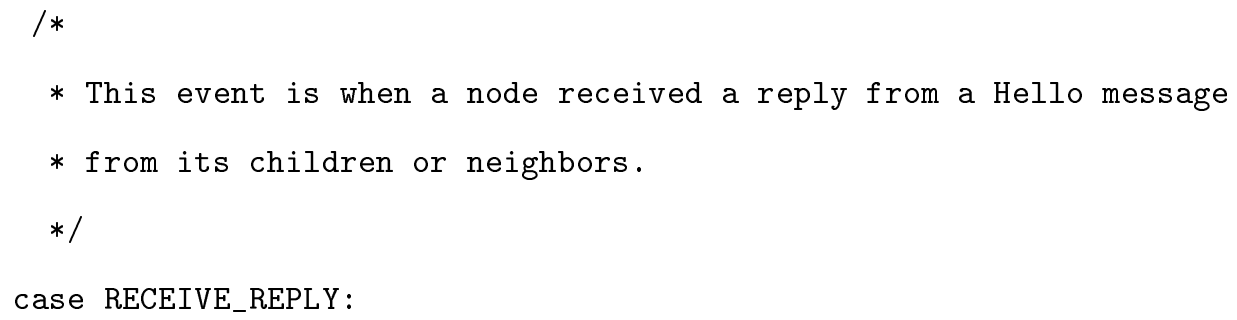




\section{Appendix A: (continued)}

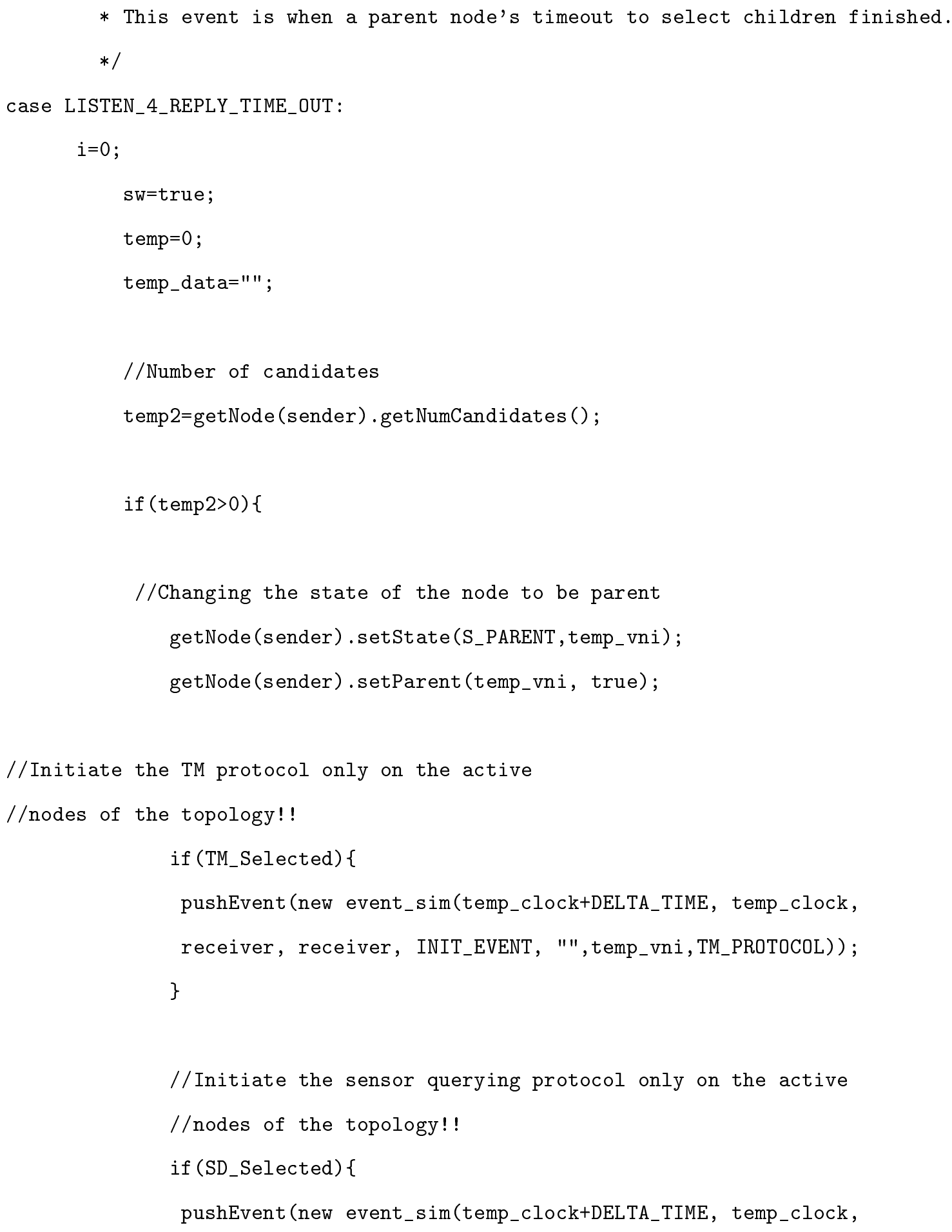




\section{Appendix A: (continued)}

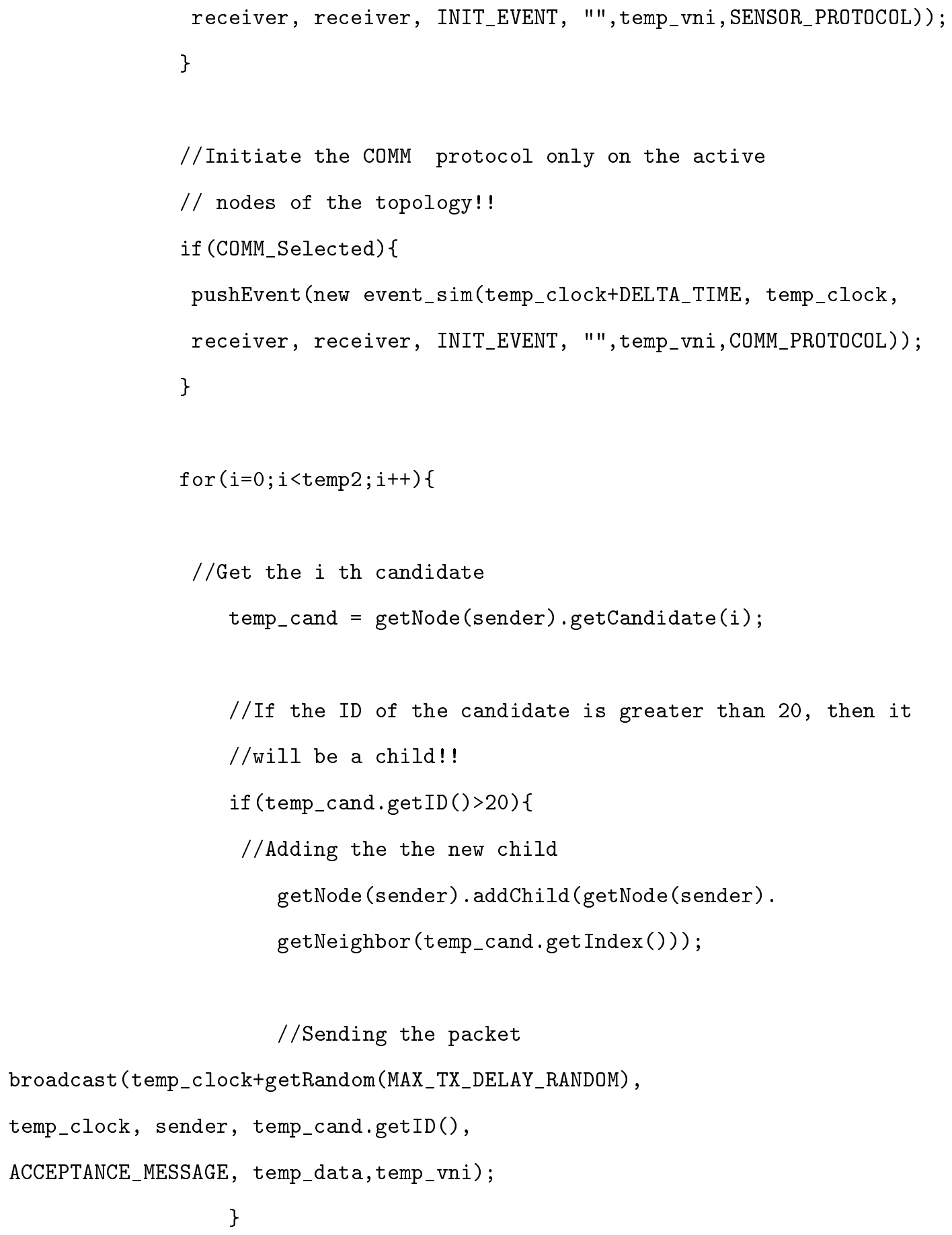




\section{Appendix A: (continued)}

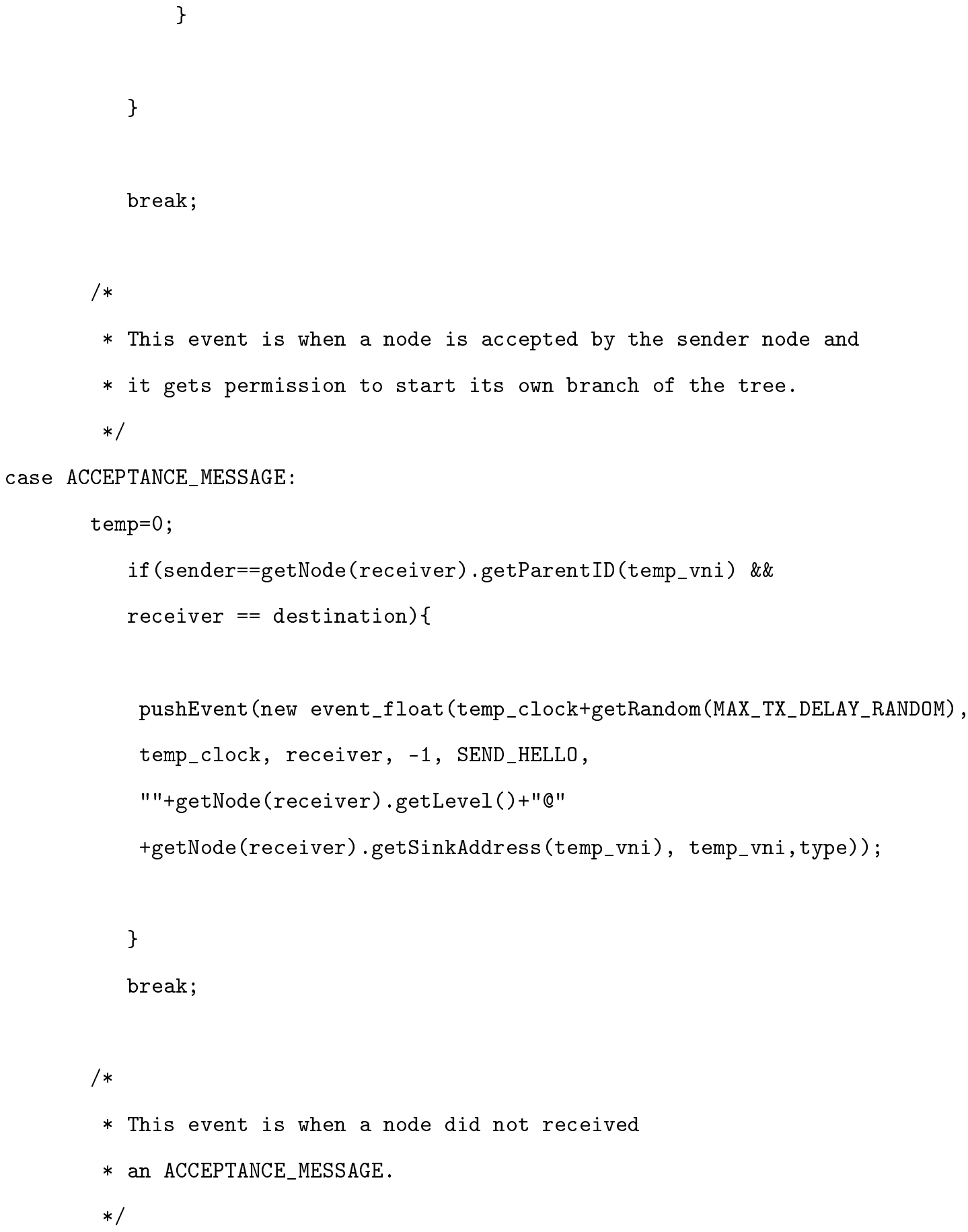




\section{Appendix A: (continued)}

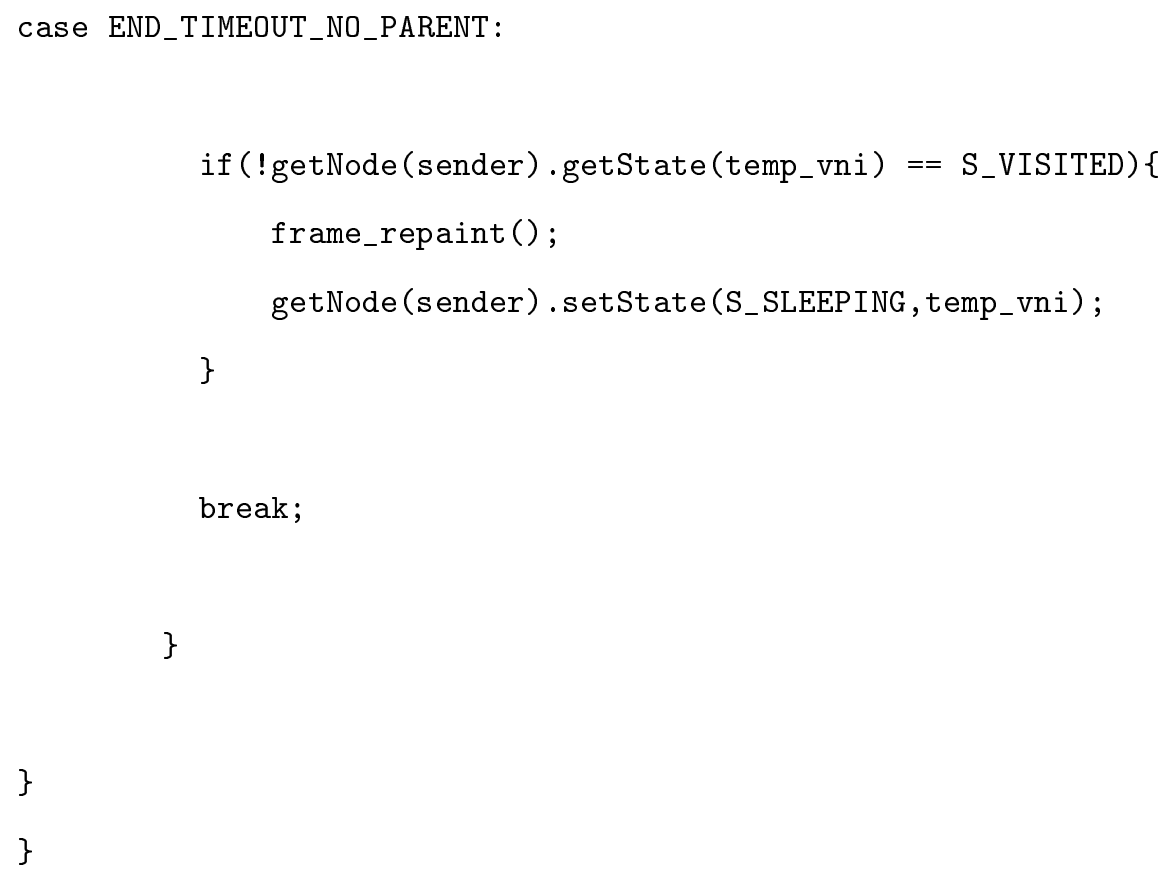

\section{SimpleTree: An Example of a Topology Construction Protocol}

In order to illustrate all these concepts, a simple example of a topology control algorithm follows. The selected example is a hierarchical topology construction protocol based on the growing a tree technique (See Section 2.2.2.1). The idea is to illustrate some common message exchange sequences, the use of timeouts, and how the modification of the status of the node modifies the execution of the protocol. The protocol works based on the following assumptions:

- The growing a tree protocol leaves every node active, except those with ID number less than 20 .

- The nodes have no information about their position and have no list of neighbors. 


\section{Appendix A: (continued)}

- Every node starts in a unvisited state.

- The sink is the initiator of the process.

- The protocol ends when every node is in active mode or in sleeping mode.

- The initial topology is connected.

The protocol, step by step, works as follows:

- The sink node initiates the protocol sending a Hello message. It includes its address and level (number of hops from the sink), which in the case of the sink it is equal to 0 . The sender node programs a timeout to stop listening for answers from its neighbors.

- All unvisited nodes on the transmission range of the sender node that received the Hello message answer back with a Reply message, set the sender as their default gateway, and change their status to In-Process mode. The receiver nodes at the same time set a timeout in case they do not receive an acknowledgment from their default gateway (the sender node).

- Once the timeout of the sender expires, this node checks the list of neighbors that answered back with the Reply message. If the sender node did not receive any answer back, it turns itself off and changes its status into a Sleeping mode. If the sender node received at least one answer back, it goes into Active mode and sends an unicast message to each one of them in order to let them know they were selected. All neighbors except those with ID number less than 20 will receive this unicast message. 


\section{Appendix A: (continued)}

- Once a node receives the confirmation message from its default gateway, it waits a random amount of time and sends its own Hello message to discover new unvisited nodes.

- If the timeout of a node in In-Process mode expires, it means that the node was not selected, so it turns itself off and goes into Sleeping mode.

- Once the node finishes its process and ends up in an Active mode, it starts the topology maintenance, sensor and data management, and communication protocols.

Although describing a protocol in words is useful for understanding its operation, they are more rigorously defined by Finite State Machines, especially in those cases where nodes change several times from one state to another during the execution of the protocol, and by a timeline of message exchanges. For this example protocol, these diagrams are shown in Figure A.2.

The first part of the algorithm is a HELLO-REPLY sequence that is used by many protocols in the neighborhood discovery process. The message sequence is simple: one message announces the presence of a node, and a set of nodes, whose size is unknown, answer back with a reply message. Given that the initiator has no idea of how many nodes are within its transmission range, it waits for a certain amount of time. This timer can be static (a fixed value or a random value defined on the fly) or dynamic (value is changed after the reception of a new reply message).

The second part of the protocol consists of the selection and notification processes. In this case, the sender node selects the next generation of possible active nodes based on a policy (all nodes with ID less than 20), and notifies them one by one using unicast messages. 


\section{Appendix A: (continued)}

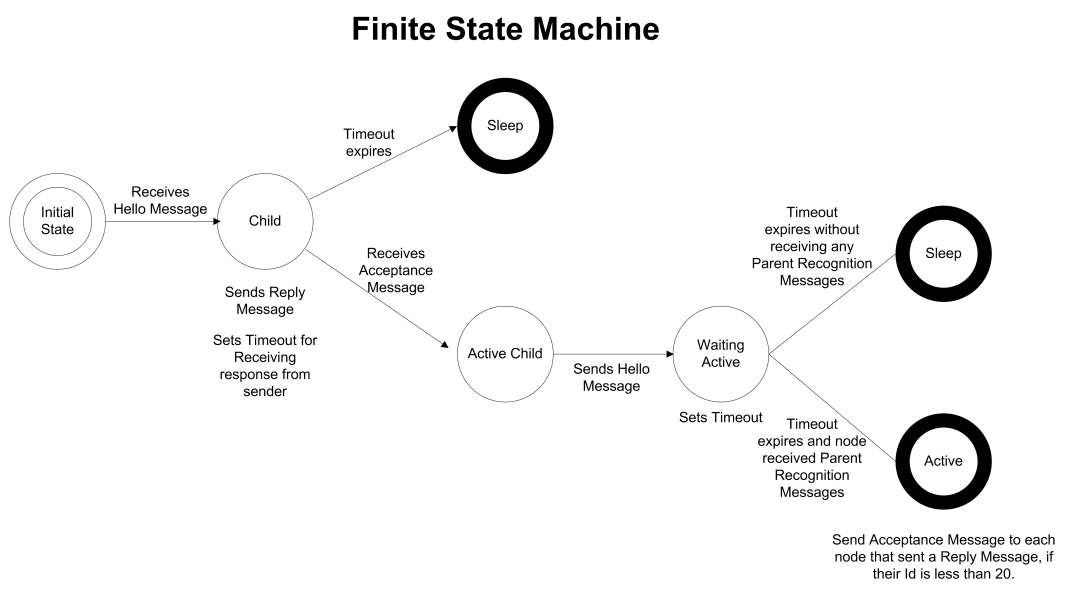

(a) Finite state machine.

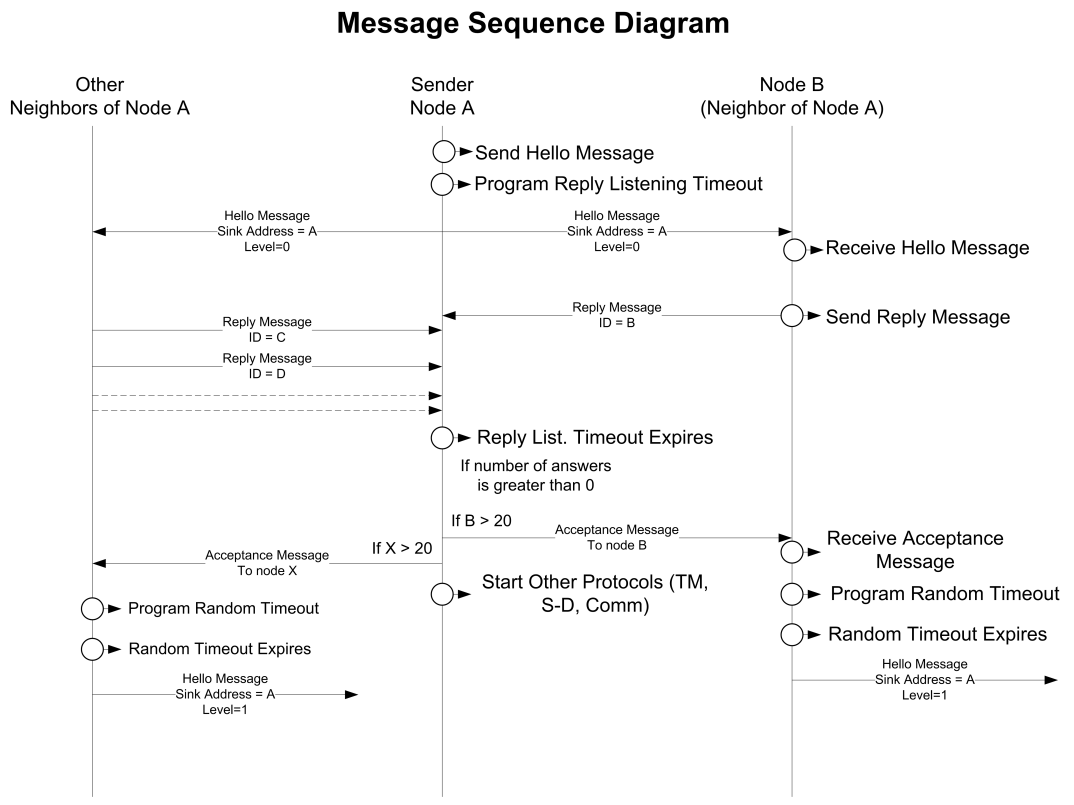

(b) Message sequence diagram.

Figure A.2: Useful diagrams to design a communication protocol. 


\section{Appendix A: (continued)}

The third part is the initiation of the other protocols. Remember that topology construction protocols are only used to reduce the size of the initial topology, but they do not necessarily take care of maintaining this topology during its operation, or send data messages to the sink. If the user is at the early stages of the protocol design, running experiments with the topology construction protocol only is very convenient; however, for more complex experiments that include network lifetime measurements it is necessary to start the topology maintenance protocols.

\section{How to Use Atarraya}

Atarraya offers a variety of options for designing and experimenting with topology control algorithms. This section provides an in-depth user guide on how to use the simulation tool and all its available options.

The first step is to have a clear understanding of the simulation scenarios to be run. Here, the user needs to know in advance which protocols he or she wants to use; whether the experiment is just a preliminary test or an exhaustive performance evaluation; what is the nature of the topologies that she is planning to use; what type of statistics are needed, and

so forth. In this section, these questions are answered in order for a user to create and run successful simulations with Atarraya.

\section{Selection of the Protocols}

Atarraya includes four types of protocols: Topology construction, topology maintenance, sensor-data management, and communication-routing protocols. Atarraya can be set to 


\section{Appendix A: (continued)}

work in either of the following two modes related to topology control: Topology construction only, or All protocols. The first mode is designed to test a specific topology construction algorithm and measure the initial reduced topology that the algorithm produces. In order to select this mode, the user only needs to select a topology construction protocol. The second mode is intented to test not only the reduced topology but the lifetime of the network, based on the combination of all the protocols, i.e. topology construction and topology maintenance. In order to select the second mode the user needs to select a protocol in each of the protocol categories, i.e. select a topology construction and a topology maintenance protocol, otherwise Atarraya will not allow the user to run the experiment.

The topology construction protocols included in Atarraya are based on algorithms presented in published papers. Currently, Atarraya includes the algorithms evaluated in this book, i.e. A3, EECDS, and CDS-Rule-K. Although all types of topology construction protocols might be implemented, such as those based on changing the transmission range of the nodes, hierarchical protocols, cluster-based protocols, etc., the current version of Atarraya includes only those based on the Connected Dominating Set concept. In addition, and for the sake of comparison with a wireless sensor network without topology control, Atarraya offers a protocol that does not reduce the topology at all, called JustTree. The only service that this protocol provides is the creation of a forwarding tree to implement the constant gateway forwarding protocol.

Atarraya also includes all the topology maintenance techniques included in Chapter 6 of this book. They are generic algorithms that work with any of the topology construction protocols. The simplest topology maintenance protocol included in Atarraya is the No 


\section{Appendix A: (continued)}

Topology Maintenance protocol, which does nothing to maintain the initial topology, but monitors it until it dies. The protocol is in charge of informing Atarraya when this event happens. In Atarraya, the termination policy is defined as the moment at which the sink stops receiving information from the nodes.

The sensor-data management protocol models the behavior of the sensors, regarding variables like data transmission frequency, data aggregation policies, etc. Atarraya provides a simple protocol for sending and receiving messages without data aggregation. Nodes transmit data packets at predefined times, and forward every received data packet based on the forwarding policy explained in the following paragraph.

Although communication or routing protocols are not the focus of this simulator, some kind of routing procedure is necessary so packets can reach the sink. Given that the topology control protocols implemented in Atarraya are designed to produce a tree-like reduced topology, the tool provides a very simple forwarding algorithm that allows packets to reach the sink node: The constant gateway forwarding protocol. In this protocol, packets are always forwarded to a default gateway unless the destination of the packet is a direct neighbor, in which case it will be sent directly to that node. In a tree-like topology, the gateway of a node is simply its parent node. In this fashion, the packet will finally reach the sink node, since it is the root of the tree.

Atarraya does not include any routing protocol, but defines a data structure that models a routing table. This data structure allows users to develop more advanced routing protocols than the one currently implemented. In addition, the routing table can store a limited amount of packet sequence numbers (events have a field for this purpose) that allow the

implementation of other forwarding algorithms like flooding-based protocols, or save the 


\section{Appendix A: (continued)}

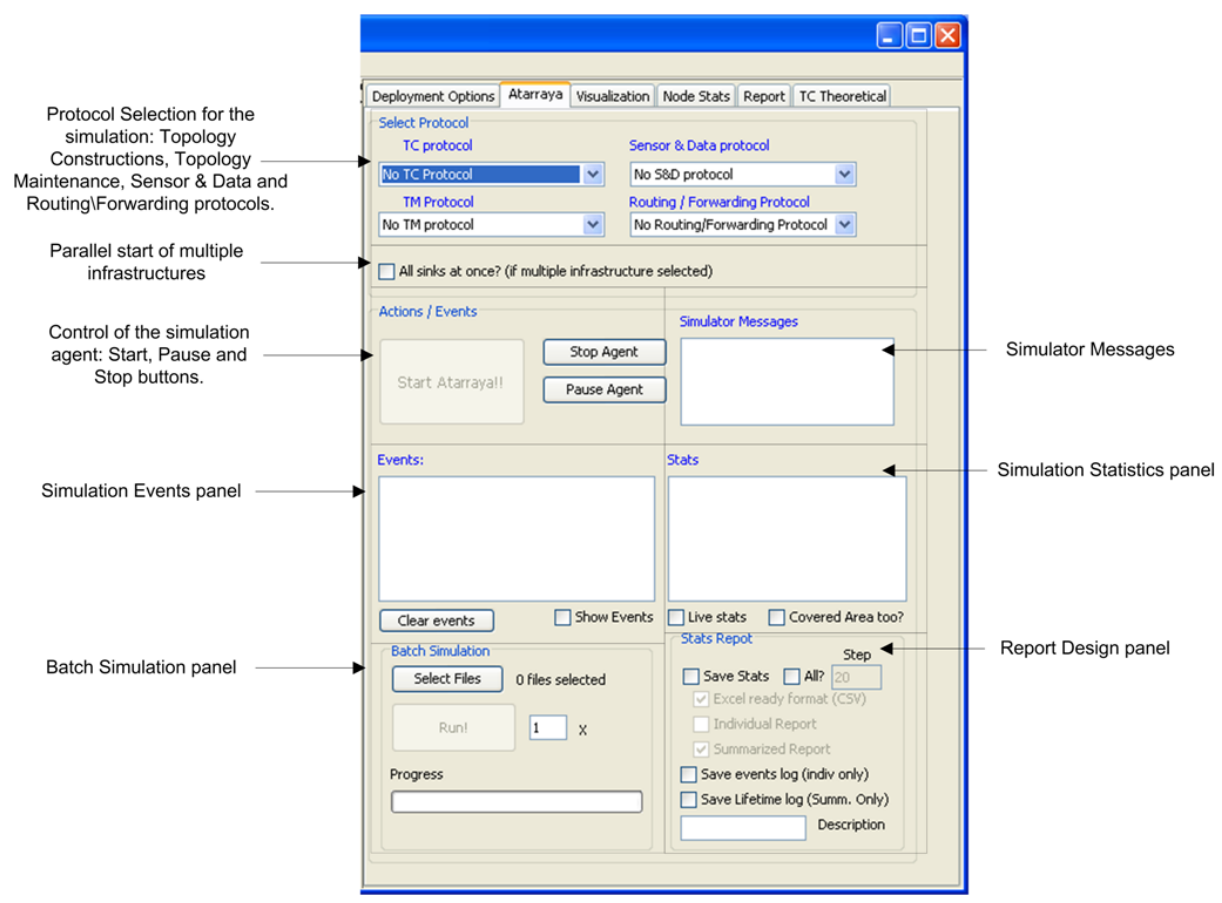

Figure A.3: Simulation control panel.

last versions of the routing protocol information packets, like routing tables on a Distance

Vector protocol, or the last neighborhood information in a Link-State protocol.

In Atarraya's graphical user interface, the panel named Atarraya presents the available protocols. In addition, this panel contains the controls for the simulation agent, the report configuration options, simulation events and statistics panels, and the batch simulations controls. This panel is shown in Figure A.3.

\section{Other Protocols}

In order to write this book, several experiments were run to validate analytically derived equations to show special effects or behaviors of topology control algorithms. This sec- 


\section{Appendix A: (continued)}

tion describes five tools included in Atarraya that are very well suited for educational purposes, as they allow users to:

- Calculate the Critical Transmission Range (CTR) based on the formulas of PenroseSanti and Santi-Blough.

- Reproduce the experiment to obtain the Giant Component figures: Greatest Connected Component and Ratio of Connected Topologies.

- Reproduce the experiment that proves connectivity of the CTR formula of SantiBlough for 2 dimensional deployments.

- Calculate the Minimal Spanning Tree on a given graph and provide the sum of the selected edges.

- Save the neighborhoods of a graph in a file.

The first three tools were utilized in Chapter 2. The user can reproduce those experiments with different parameters if so desired. The Minimal Spanning Tree of a graph is still a classical tool for graph analysis - Prim's algorithm was implemented. Regarding the last tool, the information provided by it can be used to define and solve linear programming optimization problems on graphs, like finding a minimal set cover of the graph. The panel that contains all these tools is shown in Figure A.4.

\section{Energy and Communications Model}

In the design of Atarraya simplicity and focus on reaching a better understanding of the behavior of the topology control algorithms was embraced. As such, several assumptions 


\section{Appendix A: (continued)}

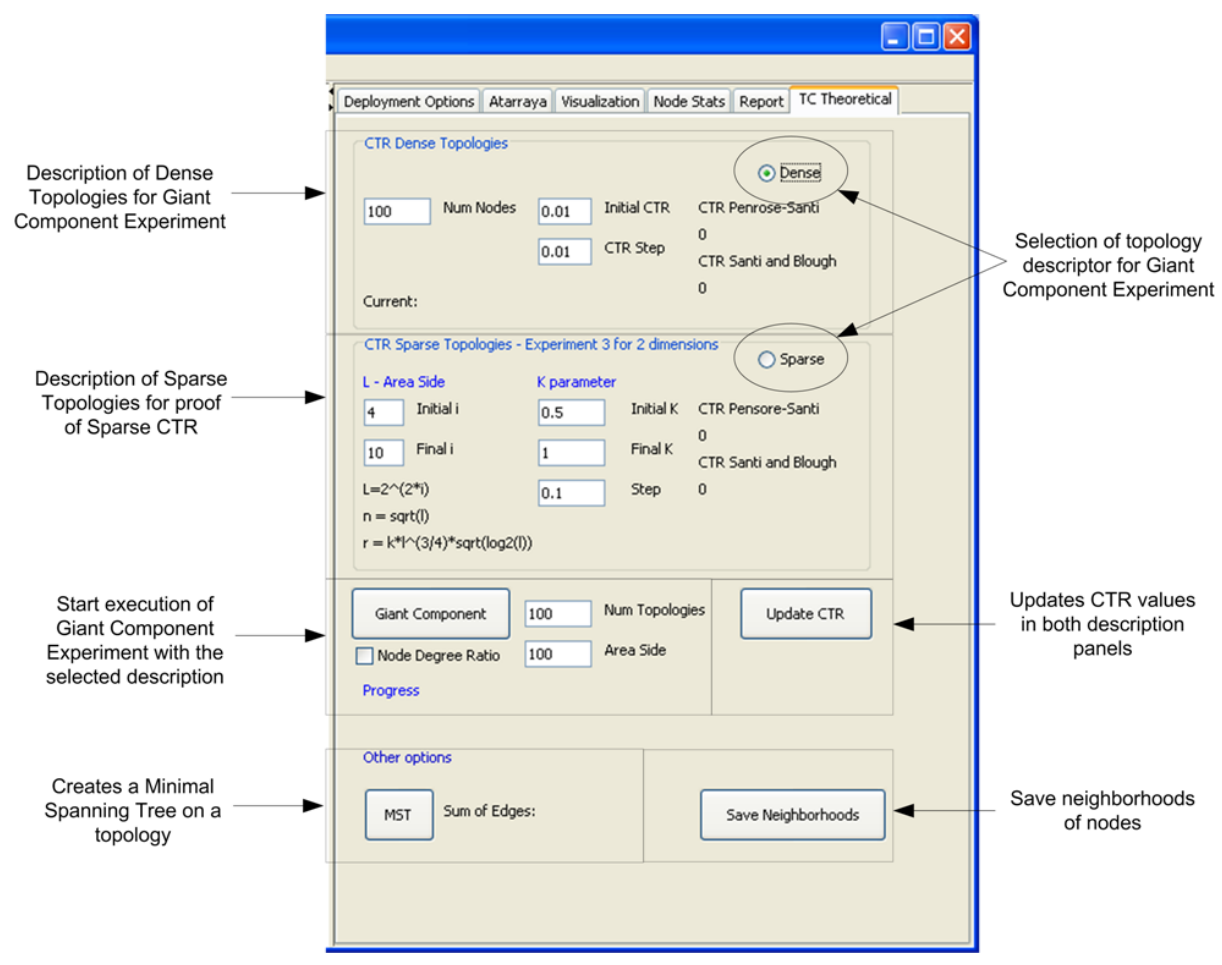

Figure A.4: Other protocols for educational purposes. 


\section{Appendix A: (continued)}

were made to make the simulator simpler while still good enough for achieving its main objective. These assumptions are related to the energy and the communication models utilized in the tool.

The energy model included in Atarraya is based on the following formulas, taken from [55]:

$$
\begin{array}{rr}
E_{T X / b i t}= & E_{\text {elect }}+\left(E_{\text {amp }} \cdot\left(\pi r^{2}\right)\right) \\
E_{R X / b i t}= & E_{\text {elect }}
\end{array}
$$

In addition, Atarraya also makes the following assumptions:

- During the idle time, a node does not spend energy. Even though this assumption has been proven untrue because being idle might be as costly as receiving data, this is still an assumption that can be done in most experiments, since the most important factor is the overhead in terms of message exchange and its associated cost.

- The nodes are assumed to have one radio for general messages and a second radio for control messages: The main radio is used in all operations when the node is in active mode, and the second one (low power cheaper radio) is used to send and receive control packets to "wake up" the main radio. Only the main radio can be turned off, which means no messages will be received and no energy will be used. The secondary radio is assumed to use half the energy of the main radio. 


\section{Appendix A: (continued)}

- The sink node has a infinite source of energy. In general, the sink node is assumed to be powered from an external source of energy.

The communication model used in Atarraya is based on the following assumptions:

- The communication range of the nodes is a perfect symmetric unit disk. If $d_{x, y} \leq$ $r_{x} \rightarrow x$ and $y$ can see each other.

- A constant bit error rate (BER) is defined for the whole network. This is a simple implementation of an error model. Whenever a packet is going to be sent, a random number is generated and compared to the message error rate (that depends on the size of the message). If the random number is greater, the message is received, otherwise it is lost. The default value for the BER is 0 , which means there is no packet loss. No sum of partially received packets will build a complete packet.

- Atarraya assumes that there exists a Data Link Layer that deals with packet losses and retransmissions, but it does not model this. In order to model some of the consequences of the operations of the MAC layer, the packets are delayed a random amount of time in order to model delays occurring due to retransmissions, contention, etc. The variable that defines the maximum delay value can be found in the constants interface, by the name of $M A X \_T X \_D E L A Y \_R A N D O M$. The default value for this variable is 0.2 time units.

\section{Type of Experiments}

Atarraya offers two types of experiments: single topology based, that uses the visual representation of the topology, and the batch execution mode that simulates a large set 


\section{Appendix A: (continued)}

of topologies. The single topology based type is good for protocol design and debugging. The batch type is made for full scale evaluation and analysis.

During the protocol design phase, it is very important to have the capability to run a small number of controlled topologies one at a time to be able to compare the results of several runs on a known scenario. This is a debugging phase where many changes are introduced in the protocol until it behaves as intended. During this process, a visual representation of the topology and the performance of the topology control algorithm is very helpful. Once the protocol has reached a stable version that has worked well in several single topologies, the protocol needs a more exhaustive test with a larger number of topologies in order to analyze its average behavior. The batch execution mode allows the researcher to run simulations with hundreds of random topologies. In this case, the visual representation of the topologies is not necessary; actually, it would slow down the simulation process.

In the single topology mode, the topology is loaded using the Load Topology item in the File menu tab, or the Deployment Options tab, which is generated by the user (the generation of topologies will be explained in the following section). In the batch execution mode, several topologies are loaded from files selected by the user. The batch execution controls are located in the Atarraya panel, as shown in Figure A.3. In order to increase the randomization of the simulation process, Atarraya introduces some noise on some common processes in the network, like message transmission delay, so each instance of a simulation, even working on the same topology, would produce different outcomes. Atarraya allows multiple replicas of each topology, which is useful 


\section{Appendix A: (continued)}

to obtain the average results and the variability of a single topology that shows the confidence of the algorithm.

\section{Structure of a Topology}

A topology in Atarraya is composed of four basic elements: Deployment area, regular nodes, sink nodes, and virtual network infrastructures or VNI. The deployment area is an abstract concept, which is useful for visualization purposes. It is a rectangle in which the user deploys the nodes of the network. In order to define the deployment area, the user needs to define its width and height.

The set of regular nodes is usually the biggest set of elements in the topology. They are in charge of monitoring the environmental variable or variable of interest, sending this information to the sink and routing packets. As with any wireless sensor devices, regular nodes are very limited in terms of resources.

The sink nodes are special nodes that, in most scenarios, are included to receive the information from all active nodes in the network. They serve as bridges between the wireless sensor network and any type of external network used to transport the sensed data to its final destination somewhere else in the Internet. In some cases they are also in charge of initiating, executing, and/or controlling the topology construction and maintenance protocols, routing protocols, etc.

Despite the fact that in real life the hardware configuration of the sink nodes is different compared to a regular wireless sensor device, Atarraya uses the same data structure to model both nodes, with the main difference that sink nodes are considered to have an unlimited amount of energy. 


\section{Appendix A: (continued)}

One contribution of Atarraya is the introduction of the concept of Virtual Network Infrastructures (VNI). This idea is an abstraction used to allow overlaying topologies over the same set of nodes. Imagine having several sink nodes on the initial topology, and that each one builds a reduced topology rooted at itself. Using this abstraction not only sink nodes can rotate, but also complete topologies. This approach was used to implement the static global topology maintenance techniques defined in Section 6.2. The initial idea was to let the network operate in the same way the lights in a Christmas tree rotate: a certain number of subsets take turns or shifts to be active during a certain amount of time, and go to sleep until the next turn.

Atarraya identifies the different VNIs with numbers from 0 to 6 , and visually with colors: Black, Red, Blue, Green, Orange, Pink, and Yellow. Each node is assumed to have a separated data structure for each VNI, so the information of each one is independent from each other. Regular nodes have no affiliation with a particular VNI, while each sink node is associated with a VNI to which it serves as a sink node. All sink nodes are assumed to be regular nodes by the VNI, that are not associated with them, keeping their characteristic of unlimited energy.

The following list contains all the available options to define the network topology:

- Sink or No Sink: Even though most wireless sensor networks contain one or more sink nodes, Atarraya allows the user to define a wireless sensor network without sinks.

- One or multiple sinks in a single VNI: Atarraya allows to define a single network with a single sink or multiple sinks. Figure A.5(a) shows this first case, which facilitates the design because there is only one active element in charge of organizing 


\section{Appendix A: (continued)}

the protocols. Nonetheless, Atarraya also allows for network designs with multiples sinks in a single network, as shown in Figure A.5(b). Including many sinks distributed in the area of deployment can be a good idea to reduce the average path length; however, having multiple sinks can also cause network partitions, especially if their structures are disjoint.

- One VNI or multiple VNIs: Having multiple VNIs is the way in which the static global topology maintenance techniques were implemented, where there are several subsets of topologies and one sink.

\section{Structure of the Nodes}

In terms of the nodes, Atarraya can manage homogeneous networks, in which all nodes have the same characteristics, and heterogeneous networks, where there is at least one node that is different from the others.

When creating a topology, the simulator works based on subsets of homogeneous nodes. Each set defines a family of nodes that share the same characteristics. For example, the user can define a set of "weak" nodes with low transmission range and low energy, and a set of "powerful" nodes with high transmission range and higher energy. Atarraya also allows for different random distributions for the location and energy of each group of nodes, that will allow the user to create denser zones in the topology, or zones with nodes that have less energy.

The data structure that models these families of nodes is called Creation Words. These creation words are created based on the values defined on the Deployment Options panel 


\section{Appendix A: (continued)}

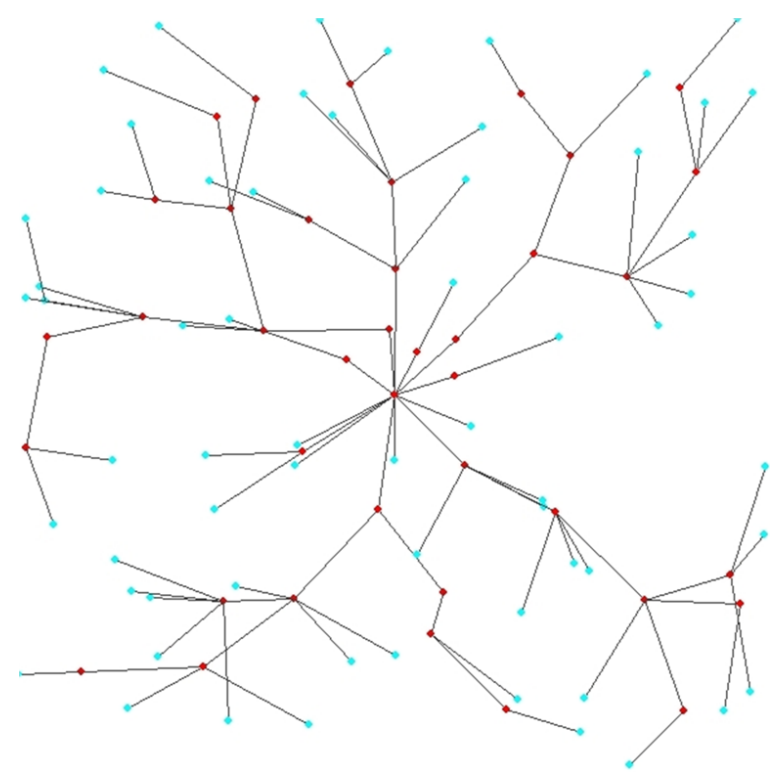

(a) Single VNI, single sink.

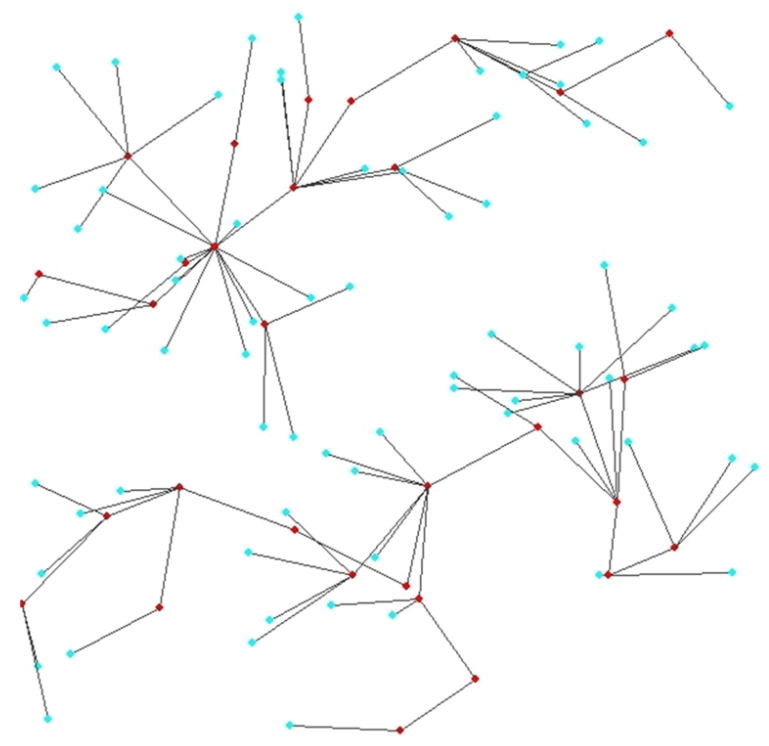

(b) Single VNI, multiple sinks.

Figure A.5: Different topology designs generated by Atarraya. 


\section{Appendix A: (continued)}

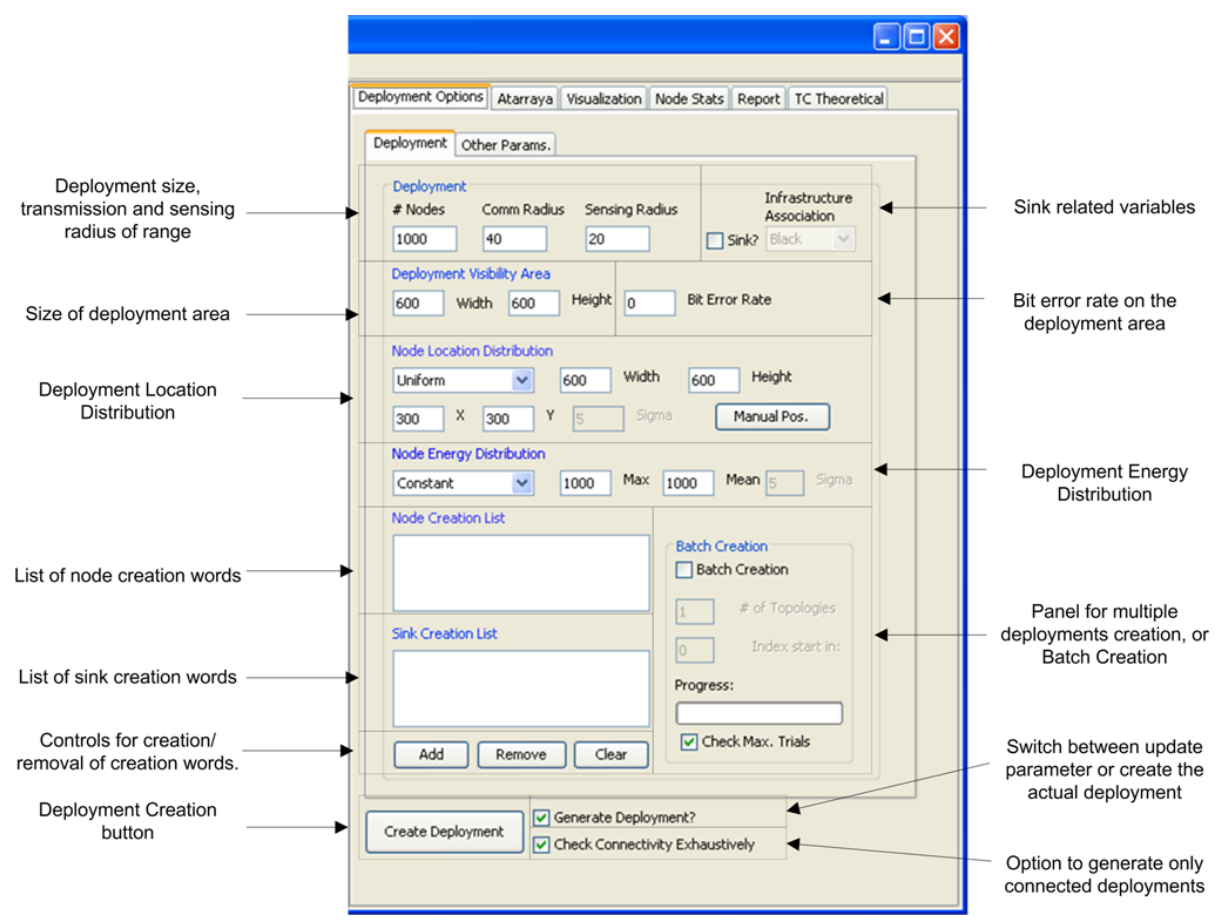

Figure A.6: Deployment definition panel.

in the simulator (Figure A.6). The user can create as many creation words as desired:

these families can model from a single node to the complete set of regular nodes. In the panel there are two list boxes where the creation words are stored until the topology is created: The regular nodes list (top) and the sink creation words (bottom). There are three buttons for adding a new creation word, removing a selected creation word, and clearing both list boxes.

The parameters that can be defined in a homogeneous family of nodes are:

- Number of nodes.

- Communication radius.

- Sensing radius. 


\section{Appendix A: (continued)}

- Size of the area of deployment.

- Position distribution:

- Uniform, with center in $(x, y)$ and the limits defined by the deployment area parameters.

- Normal with center in $(x, y)$, requiring mean and standard deviation of the location.

- Grid H-V: cover the deployment area with a grid where nodes are connected of its horizontal and vertical adjacent nodes in the grid. Number of nodes is not a parameter for this option. Distance between the nodes is the communication radius, comm $_{\text {radius }}$.

- Grid H-V-D: cover the deployment area with a grid where nodes are connected of its horizontal, vertical and diagonal adjacent nodes in the grid. The number of nodes is not a parameter for this option either. The distance between the nodes is comm $_{\text {radius }} \sqrt{2}$.

- Constant position at $(x, y)$.

- Center of area, based on the size of the deployment area parameters.

- Manual: press the button and click on the panel to select the the position of the node, as many times as defined on the number of nodes of the subset.

- Energy distribution:

- Constant value.

- Normal, requiring mean and standard deviation of the energy function. 


\section{Appendix A: (continued)}

- Poisson, requiring lambda of the energy distribution.

- Uniform, requiring maximum of the energy function.

- Inter-query time: Frequency of querying the sensor for readings. This parameter is valid only when a sensor-data protocol is selected.

In the case of the sink node set, two extra parameters must be defined:

- Sink?: select the check box if you want the nodes in the set to be included as sink nodes.

- VNI Selection: select the VNI identifier associated to the sinks of the set.

Atarraya allows the use of a metric for comparing nodes can be seen in a good number of protocols. Examples of metrics, as seen in the protocols in previous chapters, are usually related to energy, distance, ID of the node or even a random number. Atarraya allows the user to define its own metric based on its preference. In addition, it allows the use of two metrics: a primary and secondary metric. When using 2 metrics, there are different options of how to use the metrics:

- Metric1 Only (This mode is used if only one metric is needed on the protocol).

- Metric2 Only

- Metric1 Primary: the values will be grouped in blocks by Metric1, and sorted internally on each block by Metric2.

- Metric2 Primary: the values will be grouped in blocks by Metric2, and sorted internally on each block by Metric1. 


\section{Appendix A: (continued)}

- Linear combination of metrics: The node can obtain the linear combination of the 2 metrics, providing the respective weights to the primary and secondary metrics. The default values are 0.5 for both weights, so a regular average will be obtained. $\frac{((W p * \text { Metric } 1+W s * \text { Metric } 2))}{((W p+W s))}$

- Wp: weight of primary metric

- Ws: weight of secondary metric

Atarraya also includes some other special purpose variables, as follows:

- Inter-query time: Time period between reading the sensor and transmitting the data packet to the sink node.

- Inter-reset time: Time period between the time-triggered topology maintenance protocols.

- Energy threshold: Energy percentage differential used to invoke energy-triggered topology maintenance protocols. Every time the energy of a node crosses this energy threshold value, since the last invocation of the topology maintenance protocol, the node will invoke the protocol again. For example, if the battery is fully charged and the energy threshold is set to 0.10 , the node will invoke the protocol for the first time when $90 \%$ of its energy is consumed.

All these options can be found in the Deployment Options tab, under the tabs named Main Parameters and Other Parameters. The Deployment Options and Deployment's other options panels are shown in Figures A.6 and A.7. 


\section{Appendix A: (continued)}

Dual Metric Sorting Method Selection, and Size of bins for primary metric sorting

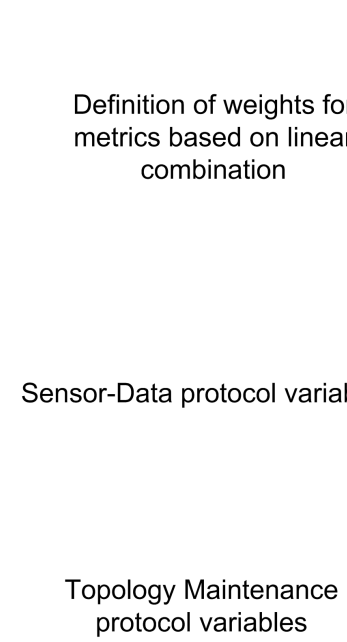

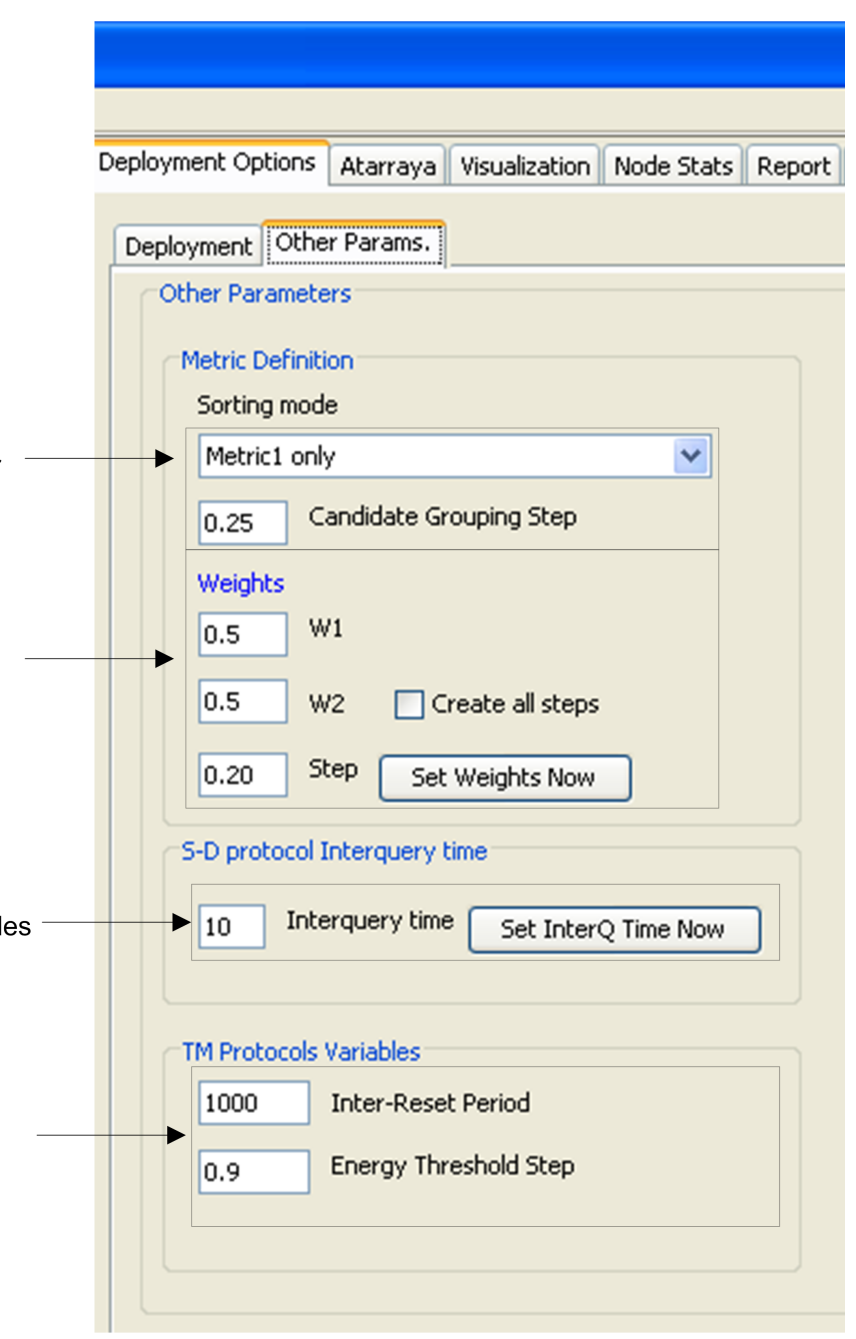

Figure A.7: Other parameters for deployment definition. 


\section{Appendix A: (continued)}

\section{Simulation Results}

Atarraya offers three types of result logs that can be obtained from a set of experiments:

General statistics, network lifetime, and the simulation event Logs. The general statistics $\log$ can register the state of several variables in a periodical manner, or provide just one snapshot of the network at the end of the simulation. The most usually consulted variables are:

- Simulation clock.

- Number of nodes.

- Number of sink nodes.

- Number of active nodes.

- Number of dead nodes.

- Average node degree.

- Average level of nodes (if level is used by the protocol).

- Number of messages sent and received.

- Number of data messages received by the sink.

- Energy on the tree, energy spent, etc.

- Area of communication coverage. 


\section{Appendix A: (continued)}

These values are stored in a text file where each individual row contains a snapshot of the variables of the network in the moment at which data was collected. If a experiment includes more than one topology, the user can decide if the data is going be stored individually per execution, or if all the results are going to be summarized in a single file. The usual format in which data is presented is in comma separated values (.csv), which is readable by most data analysis programs, like Excel, Matlab, etc. However, if the user does not want the results to be saved in files, the Report panel has a text area that holds the statistics generated by the experiments in csv format. If just one topology is simulated, the user can use the Stats text area in the Atarraya panel, which presents the results in plain text format. Figure A.8 shows one example of the simulation results generated by Atarraya.

The network lifetime log registers the status of the active topology. This log stores information every time the topology maintenance protocol is invoked, or when a node dies. This specific log cannot be stored in individual files per execution; it is stored in a summarized format in csv format.

The information stored in the network lifetime log by a single topology is represented in four rows. The first row registers the moment in which the information of the network was calculated. The second row represents the number of active nodes that can still reach the sink node - those that still can provide information to the sink. This value is important because if the sink gets isolated, no matter how many active nodes remain, all of them are useless because the information they produce gets lost. In the case of having more that one VNIs, the program works with the active VNI in the nodes. The third row shows

the ratio between the number of active nodes that can reach the sink (value found on the 
Appendix A: (continued)

Clock: 36.905478263665884

\# of Nodes: 100

\# of Sinks: 1

Not Covered: 0

Ratio: 0.0

Not Visited: 0

Avg. Level: 3.603960396039604

Avg. Num. Neighb.: 7.762376237623762

Reachable. Num. Active Neighb. from Sink: 26

\# of Messages regular sent: 317

\# of Messages long sent: 26

\# of Messages regular received: 1697

\# of Messages long received: 136

\# of Lost Messages regular: 0

\# of Lost Messages long: 0

\# of Data Messages received by sink: 0

\# of dead nodes: 0

Real Parents in Tree $0=26$

Real Parents in Tree $1=0$

Real Parents in Tree $2=0$

Real Parents in Tree $3=0$

Real Parents in Tree $4=0$

Total Energy initial $=101000.0$

Total Energy final $=100947.62194912211$

Total Energy spent $=53.07221014322563$

Total Energy spent ratio $=5.185945631473989 \mathrm{E}-4$

Total Energy in tree $=25979.027257565518$

Ratio Energy $=0.25735155277515126$

Covered Area $=0.907875$

Error in simulation=no error

Figure A.8: Example of statistics generated by Atarraya. 


\section{Appendix A: (continued)}

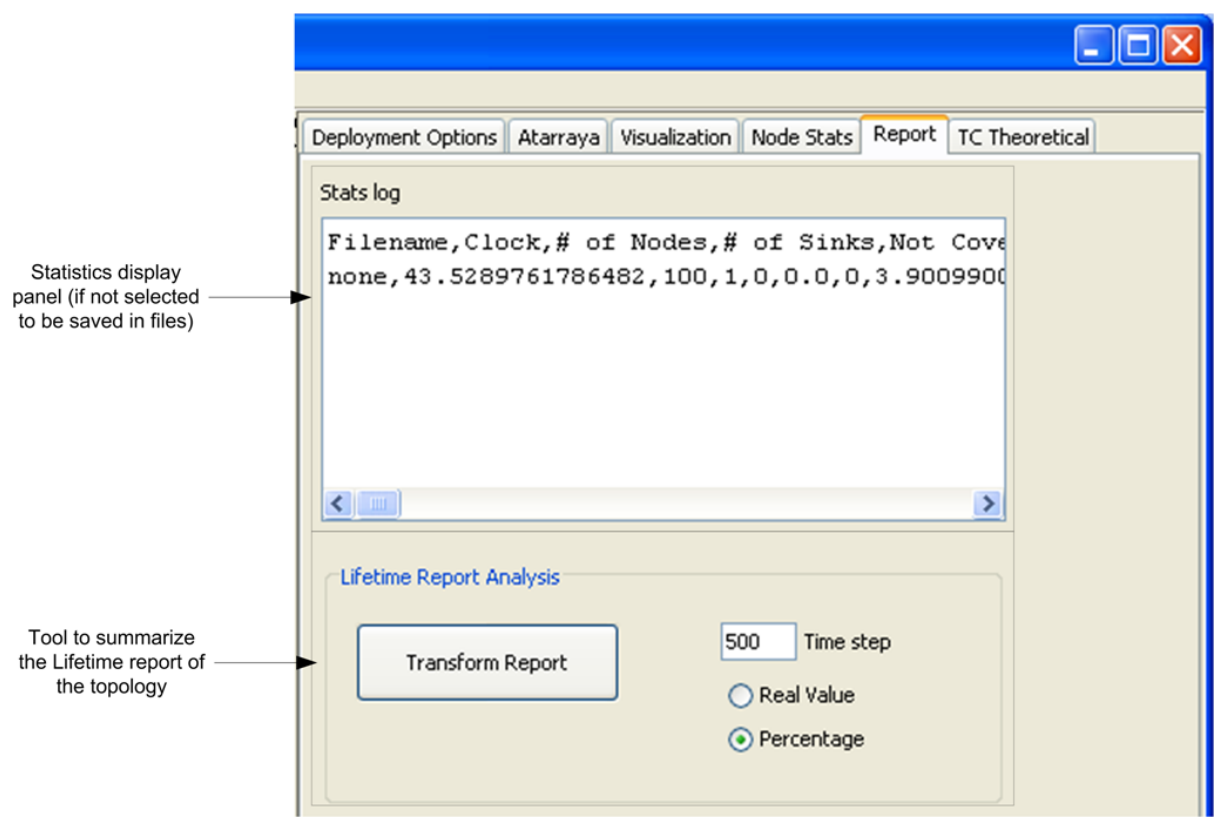

Figure A.9: Report panel.

second row) and the maximum number of active nodes. This value is the percentage of active nodes that are still alive and connected to the sink. Finally, the fourth row contains the percentage of covered area by the active nodes in the second row. This information can be very useful in order to compare efficiency between protocols, in terms of number of active nodes and real covered area.

Atarraya offers a way to summarize this log by calculating the average number of active nodes for the complete set of executions that are stored in a single file. The idea is to create a single lifetime function that represents the average number of active nodes and wraps all the individual lifetimes. Atarraya creates a discrete time line, in which the size of each cell is based on the parameter "Time step". Each cell contains the global average of all experiments during that specific amount of time. The Report panel presents the controls for this operation, as shown in Figure A.9. 


\section{Appendix A: (continued)}

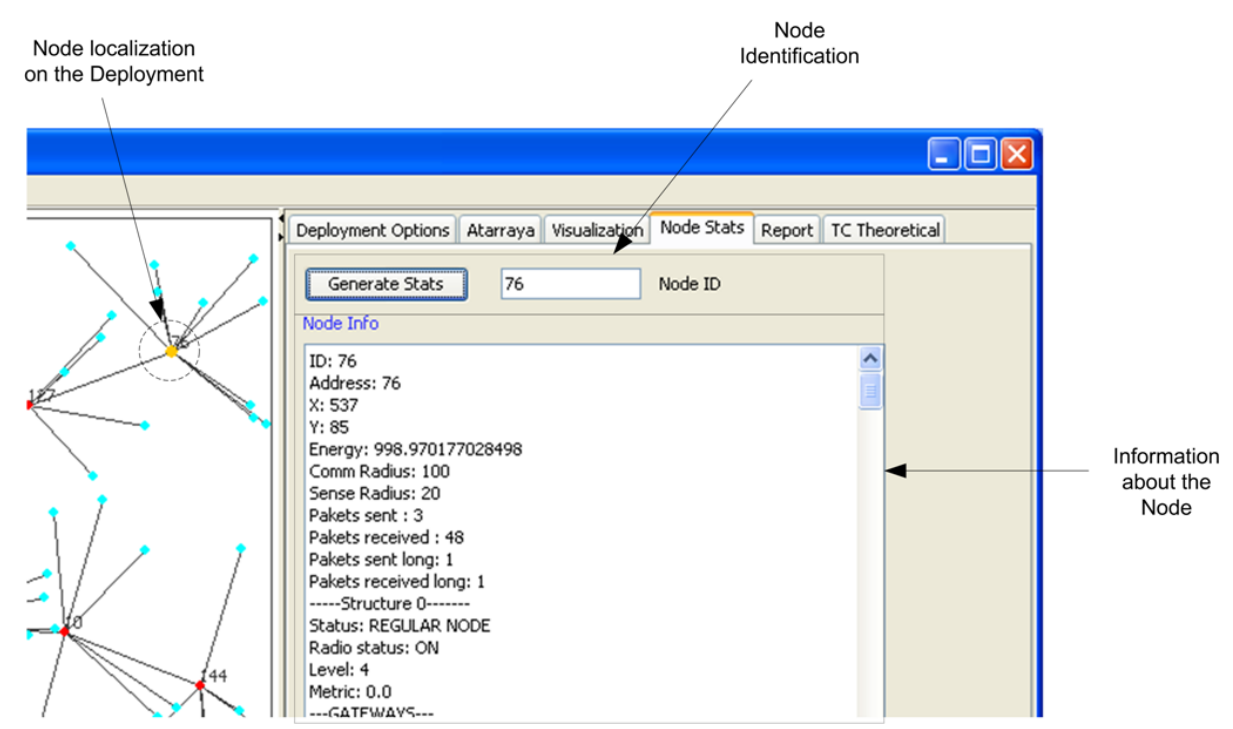

Figure A.10: Node statistics panel.

The simulation event log registers all the events generated by the simulator during the execution of a single topology. The complete set of events allows the user to debug the protocol by seeing the sequence of operations executed. This information is only available in individual files per topology.

Atarraya not only offers statistics from the complete simulation point of view, but from the individual node perspective. It is always very useful to know the status of the nodes at any given point in time to check if the algorithms are working as desired. This information can be found in the Node Stats panel, as shown in Figure A.10. In order to get the information of a node, the user can do it in two ways: dragging the mouse pointer to the desired node and clicking over it, or typing the id number of the node in the text field and press the button Generate Stats. Once the node is selected, the point in the topology turns orange and increases in size. 


\section{Appendix A: (continued)}

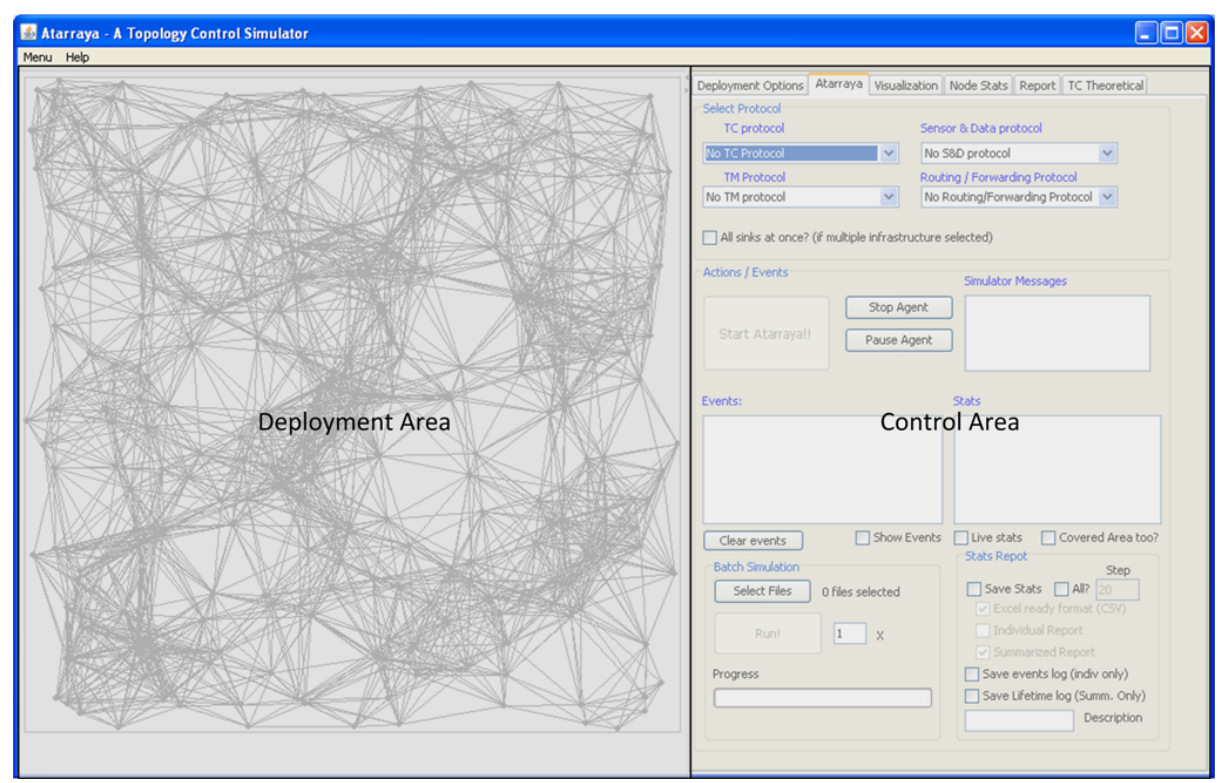

Figure A.11: Main window description.

Atarraya also allows for the visual representation of the reduced topology. The main window of Atarraya is divided in two environments: The deployment visualization area (left) and the control area (right), as shown in Figure A.11. One of the panels in the control area contains the visualization options: changing view from MaxPower graph to the reduced topology (Atarraya mode); showing the active nodes (Parent mode), communication and sensing coverage areas, and node id's; drawing a grid over the deployment the area, etc. The combination of some of these options are shown in Figure A.12, where on the left side of the figure the deployment visualization area with the different presentation options can be seen. 
Appendix A: (continued)

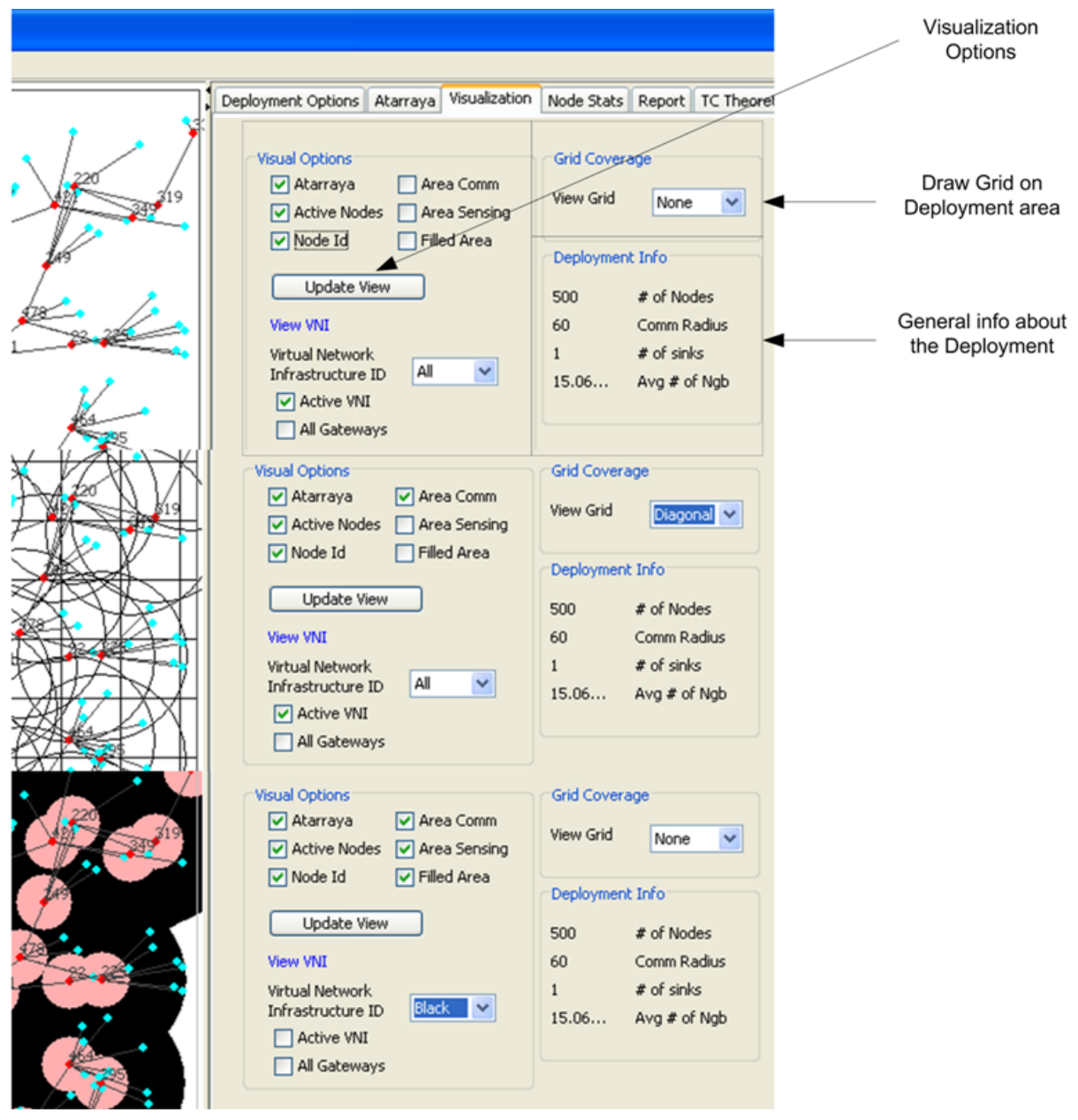

Figure A.12: Visualization control panel. 


\section{Appendix A: (continued)}

\section{Future of Atarraya}

Atarraya is a very useful tool for testing topology control algorithms, but it is still far from reaching its full potential. There is a lot of room for future improvements. For example, transmission range control topology construction protocols, local topology maintenance protocols, data aggregation algorithms, routing and forwarding protocols, 3D scenarios, etc. could be included. More complex and realistic sensing and communication models and data link layer protocols could be incorporated as well.

It is hoped that making Atarraya freely available the research community will contribute to its expansion. Maintaining Atarraya's Website with the most recent versions of the tool, its upgrades and features, for the benefit of the entire community and the research area is a major commitment. Please contact the authors with your own upgrades, suggestions, corrections, etc. The main Atarraya Website is at http://www.cse.usf.edu/ 〜abrador/Atarraya. 


\section{About the Author}

Pedro Wightman received his B.Sc. in Systems Engineering from the Universidad del Norte, in Barranquilla, Colombia, in 2004. He received his M.Sc. in Computer Science from the University of South Florida in 2007, where he is a Ph.D. candidate in the same department. Pedro worked as an adjunct instructor at the Universidad del Norte during 2004 and 2005. In 2005 he was selected to participate in the National Program of Young Researchers in Colombia, sponsored by the Colombian Institute of Science and Technology, Colciencias. In 2005, he was selected by the Universidad del Norte to participate in the Teaching Formation Program which gave him the opportunity to start his doctorate. His research interests are in the development of energy efficient topology construction and topology maintenance protocols for wireless sensor networks. He is a member of the IEEE Communication Society, and co-founder of CommNet, the Communication Networks Group at USF. 